U N I VER S TE DU Q UE B EC

Mémoire présentè à

l'Université du Québec à Chicoutimi

comme exigence partielle

de la Maittrise en Ingênierie

Par

Carine DUBUISSON

B. Génie civil

\title{
CINETIQUE DE LA CALCINATION DU COKE DE PETROLE EN ATMOSPHERE OXYDANTE
}




\section{Bibliothèque}

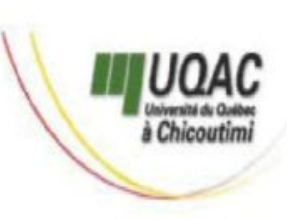

\section{Mise en garde/Advice}

Afin de rendre accessible au plus Motivated by a desire to make the grand nombre le résultat des results of its graduate students' travaux de recherche menés par ses research accessible to all, and in étudiants gradués et dans l'esprit des accordance with the rules règles qui régissent le dépôt et la governing the acceptation and diffusion des mémoires et thèses diffusion of dissertations and produits dans cette Institution, theses in this Institution, the I'Université du Québec à Université du Québec à Chicoutimi (UQAC) est fière de Chicoutimi (UQAC) is proud to rendre accessible une version make a complete version of this complète et gratuite de cette œuvre. work available at no cost to the reader.

L'auteur conserve néanmoins la The author retains ownership of the propriété du droit d'auteur qui copyright of this dissertation or protège ce mémoire ou cette thèse. thesis. Neither the dissertation or Ni le mémoire ou la thèse ni des thesis, nor substantial extracts from extraits substantiels de ceux-ci ne it, may be printed or otherwise peuvent être imprimés ou autrement reproduced without the author's reproduits sans son autorisation. permission. 
Amir.

mes parents, 


\section{REMER CIEMENTS}

Je m'acquitte bien volontiers d'un devoir de gratitude envers les nombreuses personnes qui m'ont prodigué une aide aussi bienveillante qu'efficace.

Monsieur le Professeur André CHARETTE, je tiens à vous exprimer tout particulièrement ma reconnaissance et ma gratitude pour la confiance que vous m'avez témoignée tout au long de ce travail. Vos judicieux conseils et vos encouragements ont permis le bon déroulement et l'aboutissement de cette recherche, dans une atmosphère aussi chaleureuse que consciencieuse.

Il m'est agréable d'exprimer ma gratitude au Dr. Duygu KOCAEFE, dont la gentillesse, la patience et la compétence m'auront été de précieux auxiliaires au cours de ce mémoire de maîtrise. J'espère vivement que nos contacts professionnels et privés se poursuivront bien au-delà de ce travail.

Je tiens à remercier tous les membres du Groupe de Recherche en Ingénierie des Procédés et Systèmes (GRIPS) de l'Université du Québec à Chicoutimi, et plus particulièrement Monsieur le Professeur R.T. BUI pour l'accueil qu'ils m'ont réservê au sein du groupe.

Que Monsieur Patrice PAQUETTE soit également remercié pour sa précieuse contribution en qualité de technicien du GRIPS, pour la réalisation des nombreux creusets ainsi que l'entretien et la mise au point du montage expérimental.

Je remercie également le Dr. Paul DESCLAUX pour certains calculs d'équilibre thermodynamiques qu'il a bien voulu réaliser à l'aide de son logiciel, au sein du Centre de Recherche et de Développement à Arvida (CRDA).

Je remercie le CRDA et plus particulièrement Madame Lise CASTONGUAY et le Groupe Analytique pour la préparation et les analyses structurales des échantillons.

Ma reconnaissance va également à la Société Alcan ainsi qu'à la Fondation de l'Université du Québec à Chicoutimi pour le support financier qui m'a permis de mener à bien cette étude dans le cadre de ma maîtrise. 


\section{RESUME}

De grandes quantités de coke de pétrole (coke vert) sont utilisées comme matière première pour la fabrication des électrodes en électro-métallurgie, et plus particulièrement dans l'industrie de l'aluminium. Avant d'être mis en forme avec le brai liant, le coke subit un traitement thermique à haute température, désigné sous le vocable "calcination". Au cours de la calcination, le départ des matières volatiles (hydrocarbures lourds, hydrogène et méthane) engendre des modifications structurales à l'échelle cristalline, procurant au coke calciné des propriëtés bien spécifiques, en vue de répondre aux critères de qualité des futures électrodes.

Une étude de la cinétique de dévolatilisation d'échantillons représentatifs de coke de pétrole est menée à l'aide d'un montage thermogravimétrique, dans des conditions proches de celles existant dans les fours industriels (taux de chauffage variable. pourcentage d'oxygène croissant dans le milieu). Bon nombre d'auteurs soutiennent que la dévolatilisation n'est pas influencée par la nature du milieu dans lequel elle est conduite. Notre étude confirme cette affirmation à faible taux de chauffage seulement. Par contre, lorsque le taux de chauffage croît, la dévolatilisation est retardée en atmosphère oxydante, par rapport au milieu neutre.

Un modèle cinétique permet de comparer les paramètres cinétiques globaux (ordre de la réaction, énergie d'activation) caractérisant le phénomène de dévolatilisation dans les deux milieux, sous différents taux de chauffage.

Au cours de la calcination, les produits volatils de dégazéification, une fois dégagés de la masse de coke, constituent une protection autour de la particule solide, si bien que la combustion du résidu carboné ne s'amorce qu'à haute température. La combustion des volatils et du coke est favorisée par un chauffage lent. Il réside toutefois une différence essentielle entre la combustion en phase gazeuse des volatils et la combustion solide-gaz du résidu de coke. En effet, la combustion des volatils est rapide, alors que le résidu de coke se consume lentement dans des conditions bien précises de vitesse de chauffage et de disponibilité en ce qui conceme l'oxygène comburant. Il importe davantage, dans le cadre de cette étude, de définir les conditions dans lesquelles le coke est attaqué par l'oxygène, plutôt que de s'attacher à la cinétique proprement dite de combustion du coke. 
Une analyse structurale des échantillons de coke calciné met en évidence l'influence de la nature du milieu sur les propriétés du coke. On étudie l'effet de la température de calcination, du taux de chauffage et du palier de température en fin de calcination sur l'épaisseur cristalline, la porosité apparente et la densité du coke calciné; et ce sous atmosphère contrôlée (neutre et oxydante). L'évolution de la structure interne du coke au cours du traitement thermique suit la tendance déjà observée antérieurement par d'autres auteurs; toutefois la présence d'oxygène dans le milieu réactionnel influence quelque peu les paramètres structuraux, dans des conditions bien spécifiques de température, de vitesse de chauffage ou de palier de température. 


\section{Table des matières}

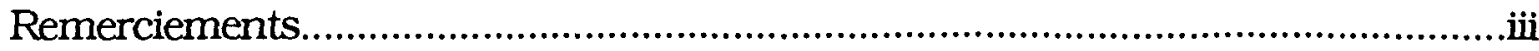

Résumé

Table des matières................................................................................................vi

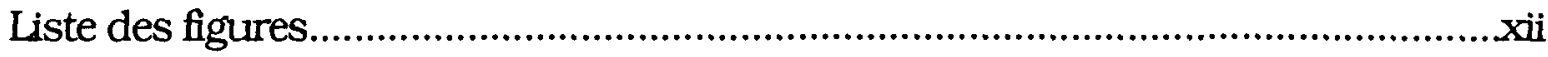

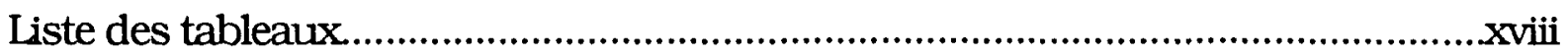

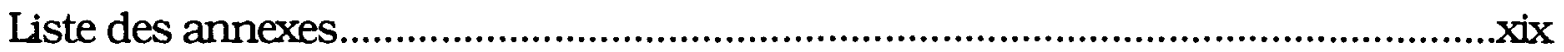

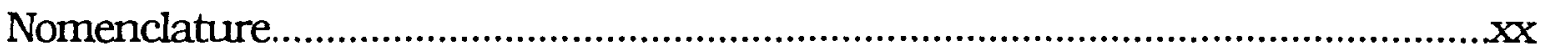

\section{INTRODUCTION}

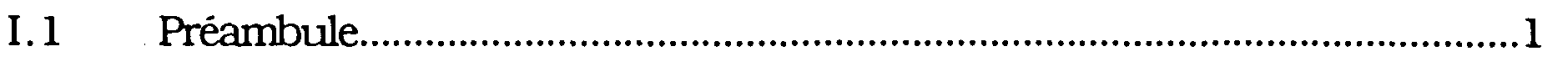

I.2 La calcination du coke: une étape importante dans le processus de fabrication des êlectrodes carbonées..................2

I.3 Phénomènes physico-chimiques se produisant au cours

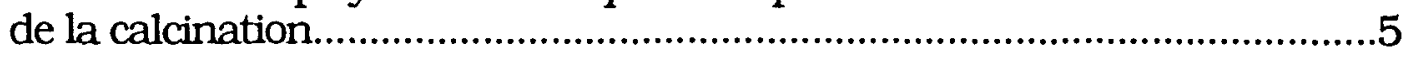

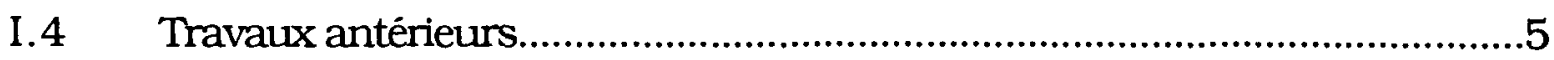

I.5 Contribution du présent travail dans la recherche........................7

I.5.1 Objectif général

I.5.2 Quelques considérations cinétiques

I.5.2.A En ce qui concerne la dévolatilisation du coke vert

I.5.2.B En ce qui concerne la combustion des volatils et du résidu de coke

I.5.3 Objectifs spécifiques

\section{TECHNIGUES D'ANALYSE ET MONTAGE EXPERIMENTAL}

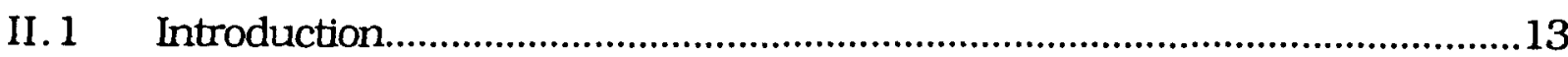




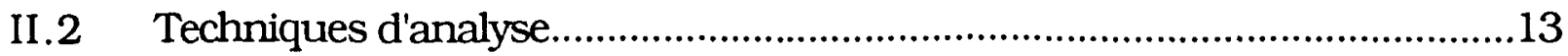

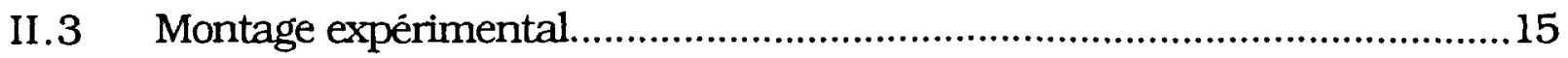

II.3.1 Description du montage

II.3.2 Conditions expérimentales

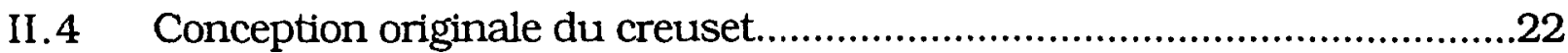

II.4.1 Quant au matériau

II.4.2 Quant à la forme

\section{PRESENTATION DES THERMOGRAMMES ET DES CHROMATOGRAMMES}

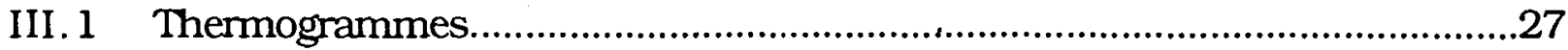

III.1.1 Correction à apporter aux résultats thermogravimétriques

III.1.1.A Force induite par le champ magnétique

III.1.1.B Poussée des gaz chauds

III.1.2 Présentation des thermogrammes

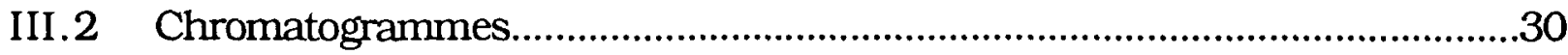

III.2.1 Correction à apporter aux résultats chromatographiques

III.2.1.A En milieu neutre

III.2.1.B En milieu oxydant

III.2.2 Calcul de la concentration des gaz par la méthode des standards externes

\section{VERIFICATION DU GRADIENT DE TEMPERATURE DANS LE CREUSET}

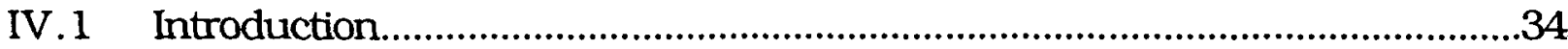

IV.2 Quelques considérations sur le chauffage par induction...................34

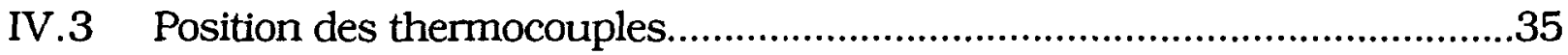


IV.4 Résultats.

IV.4.1 Gradient radial en milieu neutre

IV.4.2 Constatations en milieu neutre

IV.4.3 Gradient en milieu oxydant

IV.4.4 Constatations en milieu oxydant

IV.5 Conclusions.

V. PYROLYSE DU COKE VERT EN MILIEU NEUTRE

V.1 Introduction.

V.2 Influence de la taille des particules sur la cinétique de dévolatilisation.

V.3 Influence de la forme du creuset sur la cinétique de dévolatilisation.

V.4 Résultats thermogravimétriques.

V.4.1 Comparaison des cokes

V.4.2 Effet du taux de chauffage

V.5 Analyse des gaz

V.5.1 Comparaison des cokes

V.5.2 Effet du taux de chauffage

V.6 Modèle cinétique de dévolatilisation du coke vert en milieu neutre.

V.6.1 Méthode de calcul

V.6.2 Illustration de la méthode de calcul sur un exemple

V.7 Conclusions.

\section{PYROLYSE DU COKE VERT EN MILIEU OXYDANT}

VI.1 Introduction. 


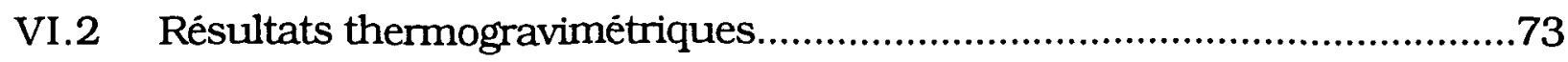

VI.2.1 Comparaison des cokes

VI.2.2 Effet du taux de chauffage

VI.2.3 Comparaison des thermogrammes obtenus en milieux neutre et oxydant

VI.3 Analyse des gaz de dévolatilisation: hydrogène et méthane.

VI.3.1 Comparaison des cokes

VI.3.2 Effet du taux de chauffage

VI.4 Analyse des gaz: monoxyde et dioxyde de carbone..........................88

VI.4.1 Comparaison des cokes

VI.4.2 Effet du taux de chauffage

VI.5 Comparaison des vitesses instantanées en milieux neutre et oxydant.

VI.5.1 En ce qui concerne les vitesses instantanées totales

VI.5.2 En ce qui concerne les vitesses instantanées relatives aux produits: hydrogène et méthane

VI.5.3 En ce qui concerne les courbes cumulatives relatives aux produits: hydrogène et méthane

VI.5.4 Estimation des quantités cumulatives d'hydrogène, de méthane et de coke consommées par la combustion en atmosphère oxydante

VI.6 Effet de la pression partielle en oxygène dans le milieu réactionnel.

VI.6.1 Effet sur la courbe thermogravimétrique

VI.6.2 Effet sur les produits de la dévolatilisation: hydrogène et méthane

VI.6.3 Effet sur les gaz: monoxyde et dioxyde de carbone 
VI.7 Calculs d'équilibre. 107

VI.7.1 Analyse des concentrations à l'équilibre

VI.7.1.A A haut taux de chauffage: $150^{\circ} \mathrm{C} / \mathrm{min}$ VI.7.1.B A faible taux de chauffage: $50^{\circ} \mathrm{C} / \mathrm{min}$

VI.7.2 Comparaison des résultats expérimentaux avec l'équilibre

VI.8 Conclusions.

\section{MODELES CINETIQUES GLOBAUX DE DEVOLATILISATION ET DE COMBUSTION DU COKE VERT}

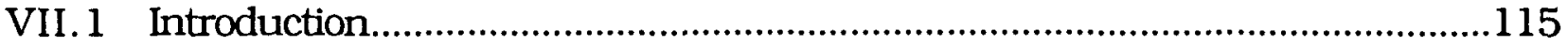

VII.2 Modèle cinétique global de dévolatilisation...................................115

VII.3 Modèle cinétique global de combustion du coke...........................120

VII.4 Conclusions..................................................................................... 124

\section{ANALYSE STRUCTURALE}

VIII. I Introduction................................................................................... 125

VIII.2 Quelques définitions................................................................. 126

VIII.3 Effet de la température de calcination.........................................126

VIII.3.1 Sur l'épaisseur cristalline

VIII.3.2 Sur la porosité apparente

VIII.3.3 Sur les densités réelle et apparente

VIII.4 Effet du taux de chauffage

VIII.4.1 Sur l'épaisseur cristalline et la densité réelle

VIII.5 Effet du palier de température à $1200^{\circ} \mathrm{C}$.

VIII.5.1 Sur l'épaisseur cristalline et la porosité apparente

VIII.5.2 Sur les densités rêelle et apparente 
VIII.6 Effet du pourcentage d'oxygène dans le milieu

VIII.7 Conclusions.

IX. CONCLUSIONS GENERALES.

BIBUOGRAPHIE

\section{ANNEXES}

Annexe I: $\quad$ Effet du taux de chauffage sur l'hydrogène et le méthane recueillis en milieu neutre pour les trois autres cokes (RTW, YPF et CONOCO)

Annexe II: Dérivée du thermogramme, valeurs instantanées et cumulatives relatives au coke TEXACO, à 100 et $50^{\circ} \mathrm{C} / \mathrm{min}$

Annexe III: Comparaison des conversions expérimentales et issues du modèle pour le coke TEXACO, à 100 et $50^{\circ} \mathrm{C} / \mathrm{min}$

Annexe IV: Comparaison des thermogrammes en milieux neutre et oxydant, pour les trois autres cokes (RTW, YPF et CONOCO).

Annexe V: Comparaison des valeurs instantanées en milieux neutre et oxydant, pour les trois autres cokes (RTW, YPF et CONOCO). 162

Annexe VI: Combustion de l'hydrogène et du méthane pour les trois autres cokes (RTW, YPF et CONOCO) en milieu oxydant.

Annexe VII : Comparaison des résultats expérimentaux avec les résultats à l'équilibre, pour le coke TEXACO à $50^{\circ} \mathrm{C} / \mathrm{min}$.

Annexe VIII : Diagramme d'Arrhénius pour la dévolatilisation des trois autres cokes (RTW, YPF et CONOCO). 


\section{Liste des figures}

Figure 1.1: Schéma général de la fabrication des électrodes carbonées dans l'industrie de l'aluminium.

Figure 2.1: Montage expérimental.

Figure 2.2: Types de sondes d'échantillonnage.

Figure 2.3: Taux de chauffage au cours des expériences de calcination.

Figure 2.4 : Schẻma du creuset de type "ouvert".

Figure 2.5 : Photo du creuset de type "ouvert".

Figure 3.1: Forces induites par un champ magnétique sur un conducteur symétrique (en coupe transversale).

Figure 3.2 : Exemple de chromatogrammes:

a) Colonne à tamis moléculaire, b) Colonne capillaire.

Figure 4.1: Position des thermocouples dans l'échantillon.

Figure 4.2: Evolution des températures à $150^{\circ} \mathrm{C} / \mathrm{min}$, en milieu neutre (100\% azote).

Figure 4.3 : Evolution des températures à $100^{\circ} \mathrm{C} / \mathrm{min}$, en milieu neutre (100\% azote).

Figure 4.4 : Evolution des températures à $50^{\circ} \mathrm{C} / \mathrm{min}$, en milieu neutre (100\% azote).

Figure 4.5: Evolution des températures à $150^{\circ} \mathrm{C} / \mathrm{min}$, en milieu oxydant ( $95 \%$ azote, $5 \%$ oxygène).

Figure 4.6: Evolution des températures à $100^{\circ} \mathrm{C} / \mathrm{min}$, en milieu oxydant ( $95 \%$ azote, $5 \%$ oxygène).

Figure 4.7 : Evolution des températures à $50^{\circ} \mathrm{C} / \mathrm{min}$, en milieu oxydant ( $95 \%$ azote, $5 \%$ oxygène).

Figure 5.1 : Comparaison des thermogrammes à $150^{\circ} \mathrm{C} / \mathrm{min}$, en milieu neutre $(100 \%$ azote $)$.

Figure 5.2: Comparaison des thermogrammes à $100^{\circ} \mathrm{C} / \mathrm{min}$, en milieu neutre $(100 \%$ azote $)$. 
Figure 5.3: Comparaison des thermogrammes à $50^{\circ} \mathrm{C} / \mathrm{min}$, en milieu neutre $(100 \%$ azote $)$.

Figure 5.4: Effet du taux de chauffage sur le thermogramme du coke TEXACO, en milieu neutre ( $100 \%$ azote).

Figure 5.5: Effet du taux de chauffage sur le thermogramme du coke RTW, en milieu neutre (100\% azote).

Figure 5.6: Effet du taux de chauffage sur le thermogramme du coke YPF en milieu neutre (100\% azote).

Figure 5.7 : Effet du taux de chauffage sur le thermogramme du coke CONOCO, en milieu neutre (100\% azote).

Figure 5.8: Comparaison de l'hydrogène et du méthane à $150^{\circ} \mathrm{C} / \mathrm{min}$, en milieu neutre (100\% azote).

Figure 5.9: Comparaison de l'hydrogène et du méthane à $100^{\circ} \mathrm{C} / \mathrm{min}$, en milieu neutre ( $100 \%$ azote).

Figure 5.10 : Comparaison de l'hydrogène et du méthane à $50^{\circ} \mathrm{C} / \mathrm{min}$, en milieu neutre (100\% azote).

Figure 5.11: Effet du taux de chauffage sur l'hydrogène du coke TEXACO, en milieu neutre (100\% azote).

Figure 5.12 : Effet du taux de chauffage sur le méthane du coke TEXACO, en milieu neutre ( $100 \%$ azote).

Figure 5.13 : Effet du taux de chauffage sur l'hydrogène du coke TEXACO, en fonction du temps.

Figure 5.14 : Effet du taux de chauffage sur le méthane du coke TEXACO, en fonction du temps.

Figure 5.15: Effet du taux de chauffage sur le monoxyde de carbone du coke TEXACO, en milieu neutre (100\% azote).

Figure 5.16: Comparaison des courbes ajustée et expérimentale pour le coke TEXACO à $150^{\circ} \mathrm{C} / \mathrm{min}$, en milieu neutre (100\% azote).

Figure 5.17: Comparaison des dérivées des courbes expérimentale et ajustée du coke TEXACO à $150^{\circ} \mathrm{C} / \mathrm{min}$, en milieu neutre.

Figure 5.18 : Valeurs instantanées pour le coke TEXACO à $150^{\circ} \mathrm{C} / \mathrm{min}$, en milieu neutre $(100 \%$ azote).

Figure 5.19: Valeurs cumulatives pour le coke TEXACO à $150^{\circ} \mathrm{C} / \mathrm{min}$, en milieu neutre (100\% azote). 
Figure 5.20 : Effet du taux de chauffage sur l'émission d'hydrogène pour le coke TEXACO, en milieu neutre (100\% azote).

Figure 5.21: Effet du taux de chauffage sur l'émission de méthane pour le coke TEXACO, en milieu neutre (100\% azote).

Figure 5.22 : Effet du taux de chauffage sur l'émission des condensables pour le coke TEXACO, en milieu neutre ( $100 \%$ azote).

Figure 5.23: Conversions pour l'hydrogène du coke TEXACO à $150^{\circ} \mathrm{C} / \mathrm{min}$, en milieu neutre (100\% azote).

Figure 5.24: Conversions pour le méthane du coke TEXACO à $150^{\circ} \mathrm{C} / \mathrm{min}$, en milieu neutre (100\% azote).

Figure 5.25: Conversions pour les condensables du coke TEXACO à $150^{\circ} \mathrm{C} / \mathrm{min}$, en milieu neutre $(100 \%$ azote $)$.

Figure 6.1 : Comparaison des courbes expérimentale et ajustée pour le coke TEXACO, aux trois taux de chauffage en milieu oxydant (95\% azote, $5 \%$ oxygène).

Figure 6.2 : Comparaison des thermogrammes à $150^{\circ} \mathrm{C} / \mathrm{min}$, en milieu oxydant ( $95 \%$ azote, $5 \%$ oxygène).

Figure 6.3 : Comparaison des thermogrammes à $100^{\circ} \mathrm{C} / \mathrm{min}$, en milieu oxydant ( $95 \%$ azote, $5 \%$ oxygène).

Figure 6.4: Comparaison des thermogrammes à $50^{\circ} \mathrm{C} / \mathrm{min}$, en milieu oxydant ( $95 \%$ azote, $5 \%$ oxygène).

Figure 6.5: Effet du taux de chauffage sur le thermogramme du coke TEXACO, en milieu oxydant ( $95 \%$ azote, $5 \%$ oxygène).

Figure 6.6 : Effet du taux de chauffage sur le thermogramme du coke RTW, en milieu oxydant ( $95 \%$ azote, $5 \%$ oxygène).

Figure 6.7 : Effet du taux de chauffage sur le thermogramme du coke YPF, en milieu oxydant ( $95 \%$ azote, $5 \%$ oxygène).

Figure 6.8 : Effet du taux de chauffage sur le thermogramme du coke CONOCO, en milieu oxydant ( $95 \%$ azote, $5 \%$ oxygène).

Figure 6.9: Comparaison des thermogrammes en milieux neutre et oxydant, à $150^{\circ} \mathrm{C} / \mathrm{min}$, pour le coke TEXACO.

Figure 6.10 : Comparaison des thermogrammes en milieux neutre et oxydant, à $100^{\circ} \mathrm{C} / \mathrm{min}$, pour le coke TEXACO. 
Figure 6.11: Comparaison des thermogrammes en milieux neutre et oxydant, à $50^{\circ} \mathrm{C} / \mathrm{min}$, pour le coke TEXACO.

Figure 6.12 : Effet du taux de chauffage sur l'hydrogène et le méthane en milieu oxydant (95\% azote, $5 \%$ oxygène).

Figure 6.13 : Effet du taux de chauffage sur l'hydrogène et le méthane pour le coke CONOCO

Figure 6.14 : Comparaison de l'hydrogène et du méthane à $150^{\circ} \mathrm{C} / \mathrm{min}$, en milieu oxydant (95\% azote, $5 \%$ oxygène).

Figure 6.15 : Comparaison de l'hydrogène et du méthane à $100^{\circ} \mathrm{C} / \mathrm{min}$, en milieu oxydant (95\% azote, $5 \%$ oxygène).

Figure 6.16: Comparaison de l'hydrogène et du méthane à $50^{\circ} \mathrm{C} / \mathrm{min}$, en milieu oxydant (95\% azote, $5 \%$ oxygène).

Figure 6.17 : Effet du taux de chauffage sur l'hydrogène du coke TEXACO, en milieu oxydant ( $95 \%$ azote, $5 \%$ oxygène).

Figure 6.18 : Effet du taux de chauffage sur le méthane du coke TEXACO, en milieu oxydant ( $95 \%$ azote, $5 \%$ oxygène).

Figure 6.19 : Comparaison des monoxyde et dioxyde de carbone à $150^{\circ} \mathrm{C} / \mathrm{min}$, en milieu oxydant (95\% azote, $5 \%$ oxygène).

Figure 6.20 : Comparaison des monoxyde et dioxyde de carbone à $100^{\circ} \mathrm{C} / \mathrm{min}$, en milieu oxydant (95\% azote, $5 \%$ oxygène).

Figure 6.21 : Comparaison des monoxyde et dioxyde de carbone à $50^{\circ} \mathrm{C} / \mathrm{min}$, en milieu oxydant ( $95 \%$ azote, $5 \%$ oxygène).

Figure 6.22 : Effet du taux de chauffage sur le monoxyde de carbone du coke TEXACO, en milieu oxydant ( $95 \%$ azote, $5 \%$ oxygène).

Figure 6.23 : Effet du taux de chauffage sur le dioxyde de carbone du coke TEXACO, en milieu oxydant ( $95 \%$ azote, $5 \%$ oxygène).

Figure 6.24 : Comparaison des valeurs instantanées en milieux neutre et oxydant, à $150^{\circ} \mathrm{C} / \mathrm{min}$, pour le coke TEXACO.

Figure 6.25: Comparaison des valeurs instantanées en milieux neutre et oxydant, à $100^{\circ} \mathrm{C} / \mathrm{min}$, pour le coke TEXACO.

Figure 6.26 : Comparaison des valeurs instantanées en milieux neutre et oxydant, à $50^{\circ} \mathrm{C} / \mathrm{min}$, pour le coke TEXACO.

Figure 6.27 : Comparaison des valeurs cumulatives en milieux neutre et oxydant, à $150^{\circ} \mathrm{C} / \mathrm{min}$, pour le coke TEXACO. 
Figure 6.28 : Comparaison des valeurs cumulatives en milieux neutre et oxydant, à $100^{\circ} \mathrm{C} / \mathrm{min}$, pour le coke TEXACO.

Figure 6.29 : Comparaison des valeurs cumulatives en milieux neutre et oxydant, à $50^{\circ} \mathrm{C} / \mathrm{min}$, pour le coke TEXACO.

Figure 6.30 : Effet du taux de chauffage sur l'hydrogène en milieu oxydant ( $95 \%$ azote, $5 \%$ oxygène).

Figure 6.31 : Effet du taux de chauffage sur le méthane en milieu oxydant ( $95 \%$ azote, $5 \%$ oxygène).

Figure 6.32 : Effet du taux de chauffage sur la combustion de l'hydrogène du coke TEXACO, en milieu oxydant.

Figure 6.33 : Effet du taux de chauffage sur la combustion du méthane du coke TEXACO, en milieu oxydant.

Figure 6.34: Combustion des quatres types de coke à $50^{\circ} \mathrm{C} / \mathrm{min}$, en milieu oxydant ( $95 \%$ azote, $5 \%$ oxygène).

Figure 6.35: Valeurs cumulatives de perte de poids, pour le calcul de la combustion du coke à $50^{\circ} \mathrm{C} / \mathrm{min}$.

Figure 6.36 : Effet du taux de chauffage sur la combustion du coke CONOCO.

Figure 6.37 : Effet de la pression partielle en oxygène dans le milieu sur le thermogramme, pour le coke TEXACO à $150^{\circ} \mathrm{C} / \mathrm{min}$.

Figure 6.38 : Effet de la pression partielle en oxygène dans le milieu sur l'hydrogène et le méthane, pour le coke TEXACO à $150^{\circ} \mathrm{C} / \mathrm{min}$.

Figure 6.39 : Effet de la pression partielle en oxygène dans le milieu sur les monoxyde et dioxyde de carbone, pour le coke TEXACO à $150^{\circ} \mathrm{C} / \mathrm{min}$.

Figure 6.40: Comparaison de l'équilibre avec l'expêrience pour l'hydrogène.

Figure 6.41: Comparaison de l'équilibre avec l'expérience pour le méthane.

Figure 6.42 : Comparaison de l'équilibre avec l'expérience pour le monoxyde de carbone.

Figure 6.43 : Comparaison de l'équilibre avec l'expérience pour le dioxyde de carbone.

Figure 6.44: Comparaison de l'équilibre avec l'expérience pour l'eau.

Figure 7.1: Cinétique de dévolatilisation du coke TEXACO à $150^{\circ} \mathrm{C} / \mathrm{min}$, en milieux neutre et oxydant. 
Figure 7.2 : Cinétique de dévolatilisation du coke TEXACO à $100^{\circ} \mathrm{C} / \mathrm{min}$, en milieux neutre et oxydant.

Figure 7.3 : Cinétique de combustion du coke TEXACO à $50^{\circ} \mathrm{C} / \mathrm{min}$, en milieu oxydant ( $95 \%$ azote, $5 \%$ oxygène).

Figure 7.4: Cinétique de combustion du coke RTW à $50^{\circ} \mathrm{C} / \mathrm{min}$, en milieu oxydant ( $95 \%$ azote, $5 \%$ oxygène).

Figure 7.5: Cinétique de combustion du coke YPF à $50^{\circ} \mathrm{C} / \mathrm{min}$, en milieu oxydant ( $95 \%$ azote, $5 \%$ oxygène).

Figure 7.6: Cinétique de combustion du coke CONOCO à $50^{\circ} \mathrm{C} / \mathrm{min}$, en milieu oxydant ( $95 \%$ azote, $5 \%$ oxygène).

Figure 7.7 : Effet du taux de chauffage sur la combustion du coke CONOCO, en milieu oxydant ( $95 \%$ azote, $5 \%$ oxygène)

Figure 8.1: Effet de la température de calcination sur l'épaisseur cristalline.

Figure 8.2 : Effet de la température de calcination sur la porosité apparente.

Figure 8.3 : Effet de la tempêrature de calcination sur la densité réelle.

Figure 8.4: Effet de la température de calcination sur la densité apparente.

Figure 8.5 : Effet du taux de chauffage sur l'épaisseur cristalline.

Figure 8.6 : Effet du taux de chauffage sur la densité réelle.

Figure 8.7 : Effet du palier de température sur l'épaisseur cristalline.

Figure 8.8 : Effet du palier de température sur la porositê apparente.

Figure 8.9: Effet du palier de température sur la densité réelle.

Figure 8.10 : Effet du palier de température sur la densité apparente.

Figure 8.11: Effet du pourcentage d'oxygène dans le milieu sur l'épaisseur cristalline.

Figure 8.12 : Effet du pourcentage d'oxygène dans le milieu sur la densité réelle. 


\section{Liste des tableaux}

Tableau 2.1: Conditions d'analyse en chromatographie gazeuse pour l'hydrogène et le méthane.

Tableau 2.2: Conditions d'analyse en chromatographie gazeuse pour les monoxyde et dioxyde de carbone.

Tableau 2.3 : Propriétés des cokes verts.

Tableau 2.4: Conditions expérimentales de calcination.

Tableau 4.1: Paramètres caractérisant l'évolution des températures en milieu neutre $(100 \%$ azote).

Tableau 4.2: Paramètres caractérisant l'évolution des températures en milieu oxydant ( $95 \%$ azote, $5 \%$ oxygène).

Tableau 5.1 : Perte de poids totale en fin de calcination $\left(1200^{\circ} \mathrm{C}\right)$ en milieu neutre $(100 \%$ azote).

Tableau 5.2 : Perte de poids totale relative à chaque produit en milieu neutre.

Tableau 5.3 : Paramètres cinétiques relatifs à chaque produit en milieu neutre.

Tableau 6.1 : Perte de poids totale en fin de calcination $\left(1200^{\circ} \mathrm{C}\right)$ en milieu oxydant $(95 \%$ azote, $5 \%$ oxygène).

Tableau 6.2: Combustion des produits volatils et du coke en milieu oxydant ( $95 \%$ azote, $5 \%$ oxygène).

Tableau 6.3: Composition du mélange pour le calcul des concentrations à l'équilibre.

Tableau 7.1: Paramètres cinétiques globaux de dévolatilisation en milieux neutre et oxydant.

Tableau 7.2: Paramètres cinétiques globaux de combustion du coke en milieu oxydant.

Tableau 8.1: Paramètres des corrélations relatives aux propriétés structurales des cokes. 


\section{Liste des annexes}

Annexe I : Effet du taux de chauffage sur l'hydrogène et le méthane recueillis en milieu neutre (100\% azote), pour les trois autres cokes (RTW, YPF et CONOCO).

Annexe II : Dërivée du thermogramme, valeurs instantanées et cumulatives, relatives au coke TEXACO, à 100 et $50^{\circ} \mathrm{C} / \mathrm{min}$.

Annexe III : Comparaison des conversions expérimentales et issues du modèle pour le coke TEXACO, à 100 et $50^{\circ} \mathrm{C} / \mathrm{min}$.

Annexe IV: Comparaison des thermogrammes en milieux neutre et oxydant, pour les trois autres cokes (RTW, YPF et CONOCO).

Annexe V : Comparaison des valeurs instantanées en milieux neutre et oxydant, pour les trois autres cokes (RTW, YPF et CONOCO).

Annexe VI : Combustion de l'hydrogène et du méthane pour les trois autres cokes (RTW, YPF et CONOCO) en milieu oxydant.

Annexe VII : Comparaison des résultats expérimentaux avec les résultats à l'équilibre, pour le coke TEXACO à $50^{\circ} \mathrm{C} / \mathrm{min}$.

Annexe VIII : Diagramme d'Arrhénius pour la dévolatilisation des trois autres cokes (RTW, YPF et CONOCO). 


\section{Nomenclature}

A Facteur pré-exponentiel d'Arrhénius [s $\left.\mathbf{s}^{-1}\right]$

$A_{i} \quad$ Aire sous le pic du composé $i$

dans l'èchantillon $\left[\mathrm{cm}^{2}\right]$

$\mathrm{A}^{\prime} \quad \quad$ Aire sous le pic du composé $\mathrm{i}$

dans le mélange étalon $\left[\mathrm{cm}^{2}\right]$

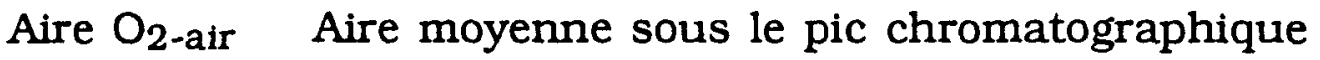

relatif à l'oxygène de l'air

$\left[\mathrm{cm}^{2}\right]$

Aire(gaz)corr Aire corrigée pour l'air infiltré,

relative au gaz analysé $\quad\left[\mathrm{cm}^{2}\right]$

a Pente de la droite de montée en

température $\quad\left[{ }^{\circ} \mathrm{C} / \mathrm{s}\right]$

b Ordonnée à l'origine $\quad\left[{ }^{\circ} \mathrm{C}\right]$

c Vitesse de chauffage $\quad\left[{ }^{\circ} \mathrm{C} / \mathrm{s}\right]$

$\mathrm{C}_{\mathrm{i}} \quad$ Concentration du composé i

dans l'échantillon [ppm]

$\mathrm{C}^{\prime}{ }_{\mathrm{i}} \quad$ Concentration du composé $\mathrm{i}$

dans le mélange étalon [ppm]

Cn Relatif aux produits condensables en milieu neutre

Co Relatif aux produits condensables en milieu oxydant

$\mathrm{D}_{\mathrm{a}} \quad$ Densité apparente $\left[\mathrm{g} / \mathrm{cm}^{3}\right]$

$D_{r} \quad$ Densité réelle $\left[\mathrm{g} / \mathrm{cm}^{3}\right]$

$\mathrm{d}_{\mathrm{o}} \quad$ Epaisseur des courants induits [m]

DTGA Dérivée de la courbe thermogravimétrique

E Energie d'activation $[\mathrm{J} / \mathrm{mol}]$

$F_{1}, F_{2} \quad$ Forces dues au champ magnétique [N]

f Fréquence

[Hz]

FR Facteur de réponse

Hn Relatif à l'hydrogène en milieu neutre

Ho Relatif à l'hydrogène en milieu oxydant

$k \quad$ Constante cinétique de réaction

$\left[\mathbf{s}^{-1}\right]$

$\mathbf{k}_{\text {diff }} \quad$ Constante cinétique en diffusion

externe

[g/(s.atm $\left.\left.{ }^{\mathrm{n}}\right)\right]$ 


\begin{tabular}{|c|c|c|}
\hline $\mathbf{k}_{\exp }$ & \multicolumn{2}{|c|}{ Constante cinétique issue de l'expérience $\left[\mathrm{s}^{-1}\right]$} \\
\hline $\mathbf{k}_{\bmod }$ & Constante cinétique issue du modèle & {$\left[\mathbf{s}^{-1}\right]$} \\
\hline $\mathbf{k}_{\mathbf{s}}$ & Constante cinétique en diffusion & \\
\hline & externe et réaction chimique & {$\left[\mathrm{g} /\left(\mathrm{s} \cdot \operatorname{atm}^{\mathrm{n}}\right)\right]$} \\
\hline $\mathrm{L}_{c}$ & Epaisseur cristalline & [Å] \\
\hline m & Ordre réel de la réaction & \\
\hline $\mathbf{M}_{\mathbf{S}}$ & Masse de solide & [g] \\
\hline $\mathrm{Mn}$ & Relatif au méthane en milieu neutre & \\
\hline Mo & Relatif au méthane en milieu oxydant & \\
\hline $\mathbf{n}$ & Ordre apparent de la réaction & \\
\hline $\mathrm{pg}$ & Pression partielle en oxygène & \\
\hline & dans le milieu réactionnel & [atm] \\
\hline $\mathrm{P}_{\mathrm{a}}$ & Porosité apparente & [\%] (en volume) \\
\hline pt & Palier de température à $1200^{\circ} \mathrm{C}$ & [min] \\
\hline $\mathrm{pO}_{2}$ & Pourcentage d'oxygène dans le milieu & [\%] \\
\hline $\mathrm{q}$ & $\begin{array}{l}\text { Vitesse de combustion } \\
\text { de la particule solide }\end{array}$ & [g/s] \\
\hline $\mathbf{R}$ & Constante des gaz parfaits & {$[\mathrm{J} /(\mathrm{mol} . \mathrm{K})]$} \\
\hline $\mathbf{r}$ & Nombre de moles de résidu final & \\
\hline $\mathrm{RD}$ & Ratio de dilution pour analyse chromatog & raphique \\
\hline Rés. & Résidu final & \\
\hline $\mathbf{s}$ & Nombre de moles de solide initial & \\
\hline Sol. & Solide initial & \\
\hline $\mathrm{T}$ & Température absolue & [K] \\
\hline $\mathrm{t}$ & Temps & [s] \\
\hline $\mathrm{T}_{\mathrm{c}}$ & Température au centre de l'échantillon & {$\left[{ }^{\circ} \mathrm{C}\right]$} \\
\hline tc & Taux de chauffage & {$\left[{ }^{\circ} \mathrm{C} / \mathrm{min}\right]$} \\
\hline $\mathrm{T}_{\mathrm{d}}$ & Température au dessus de l'échantillon & {$\left[{ }^{\circ} \mathrm{C}\right]$} \\
\hline $\mathrm{T}_{\text {ext }}$ & $\begin{array}{l}\text { Température sur la paroi externe du } \\
\text { creuset }\end{array}$ & {$\left[{ }^{\circ} \mathrm{C}\right]$} \\
\hline$T_{f}$ & Température au fond de l'échantillon & {$\left[{ }^{\circ} \mathrm{C}\right]$} \\
\hline TGA & Courbe thermogravimetrique ou thermog & ramme \\
\hline$T_{\text {int }}$ & $\begin{array}{l}\text { Température sur la paroi interne du } \\
\text { creuset }\end{array}$ & {$\left[{ }^{\circ} \mathrm{C}\right]$} \\
\hline $\operatorname{Tn}$ & Dérivée totale en milieu neutre & \\
\hline To & Dérivée totale en milieu oxydant & \\
\hline
\end{tabular}


$\mathrm{T}_{\mathrm{py}} \quad$ Température de surface lue par le

pyromètre

$\left[{ }^{\circ} \mathrm{C}\right]$

$\mathbf{v}$

Nombre de moles de produit volatil

$\mathrm{V}_{\mathrm{S}}$

Volume de solide

$\left[\mathrm{cm}^{3}\right]$

$V_{\mathrm{f}}$

Volume des pores fermés

$\left[\mathrm{cm}^{3}\right]$

$V_{0}$

Volume des pores ouverts

$\left[\mathrm{cm}^{3}\right]$

Vol.

Produit volatil

$\mathrm{X}_{\mathrm{i}}$

Fraction massique du composé i

$\mathrm{x}, \mathrm{y}$

Concentration en $\mathrm{CH}_{4}$ et $\mathrm{H}_{2}$ dans le mélange étalon

[ppm]

$\mu$

Perméabilité magnétique

$[\mathrm{H} / \mathrm{m}]$

$\rho$ Résistivité électrique

[ $\Omega . \mathrm{m}]$

$\% \mathrm{O}_{2 \text {-ech }} \quad$ Pourcentage d'oxygène infiltré dans

la seringue lors de la prise manuelle

de l'échantillon gazeux

\%alr-ech Pourcentage d'air infiltré dans la

seringue lors de la prise manuelle de

l'échantillon gazeux

\section{Indices}
air
Relatif à l'air
corr
Valeur corrigée pour l'air infiltré dans la seringue
êch
Relatif à l'échantillon gazeux
$\exp$
Valeur expérimentale
i
Relatif au composé i
$\bmod$
Valeur issue du modèle 


\section{CHAPITRE I}

\section{INTRODUCTION}

\section{I.1 Préambule}

Tous les corps organiques ont la propriété de se décomposer sous l'effet de l'élévation de la température. Cette décomposition présente toujours l'aspect d'un fractionnement. Par exemple, un hydrocarbure à taux d'hydrogène élevé, se sépare en fractions plus riches en hydrogène s'éliminant sous forme de gaz, et en un résidu plus pauvre en hydrogène. Vers $1000^{\circ} \mathrm{C}$, ce résidu est presqu'uniquement composé de carbone. Dans tous les cas, il se produit d'une part un craquage donnant des molécules légères (matières volatiles) et, d'autre part, une polymérisation forcée de la phase condensée dont la forme solide finale est désignée par le vocable "carbone".

Le coke de pétrole ou coke vert est un sous-produit obtenu par la pyrolyse $\left(1000-1400^{\circ} \mathrm{C}\right)$ des résidus du craquage des produits pétroliers. Naguère déchet de fabrication, le coke est devenu, par suite de progrès considérables dans la distillation du pétrole, une matière première que l'on fabrique aujourd'hui pour divers usages.

Une des applications importantes de ce matériau est son utilisation comme matière première dans la fabrication des anodes en électro-métallurgie, et plus particulièrement dans l'industrie de l'aluminium.

Parmi ses nombreuses qualités, la bonne conductivité électrique du coke après sa calcination, constitue un atout majeur dans son choix pour la fabrication des électrodes. 


\section{I.2 La calcination du coke: une étape importante dans le processus de fabrication des électrodes carbonées}

Le coke de pétrole renferme un pourcentage élevé de carbone (85\%) ainsi que des quantités plus ou moins importantes d'hydrogène et d'hydrocarbures légers qui s'échappent sous forme de matières volatiles au cours d'un traitement thermique à haute température. On procède donc, prealablement à son utilisation, à une calcination du coke, dont le but est de réduire l'indice des matières volatiles du coke calciné et d'en améliorer la structure cristalline ainsi que la conductivité électrique.

Dans l'industrie de l'aluminium, la calcination du coke est conduite dans des fours rotatifs où la température atteint environ $1200^{\circ} \mathrm{C}$. Après broyage et tamisage, le coke calciné est acheminé dans des malaxeurs où il est mélangé à un brai liant, pour la mise en forme des électrodes sous forme de blocs; ensuite les blocs subissent une cuisson finale. La cuisson des électrodes a pour but d'en améliorer les propriétés mécaniques et la conductivité électrique, suite au dégagement des matières volatiles du brai et à la carbonisation des parties lourdes de ce même brai.

On comprend donc aisément qu'il est primordial d'éliminer les matières volatiles du coke au cours de l'étape préalable de la calcination, car un indice élevé en volatils causerait entre autre la fissuration des blocs au cours de la cuisson des électrodes.

La figure 1.1 représente le schéma général de la fabrication des électrodes dans l'industrie de l'aluminium.

L'intêrêt que l'on porte à la calcination du coke est guidé par deux aspects essentiels de ce traitement thermique à haute température: d'une part, au niveau de la qualité des électrodes car les propriétés du coke calciné conditionnent directement la qualité des électrodes; et, d'autre part, au niveau du bilan énergétique des fours auquel participe la chaleur dégagée par les réactions se produisant au cours de la calcination (réactions autothermes).

Les conditions de calcination (vitesse de chauffage, débit d'air, etc.) et la qualitê intrinsèque du coke vert influencent les propriétés du coke 
calciné. Au cours du chauffage, le coke subit de nombreuses modifications structurales qui définiront, dans une large mesure, la qualité des futures électrodes, tant au niveau des propriétés mécaniques qu'électriques ou de leur performance.

De plus, les réactions exothermiques qui se déroulent au cours de la calcination produisent une énergie calorifique importante (dégagement des matières volatiles, combustion en présence d'air), pouvant contribuer à l'apport énergétique nécessaire au traitement thermique (auto-calcination du coke).

Une meilleure connaissance de la cinétique de dégagement des matières volatiles permet de mieux évaluer la contribution des gaz au bilan énergétique des fours. Une analyse des changements structuraux subis par le coke au cours de la calcination permet de mieux contrôler la qualité des produits finis, en l'occurrence les électrodes. 


\section{Coke vert}

(coke de pétrole)
CALCINATION $\left(1000-1200^{\circ} \mathrm{C}\right)$

Buts :

- teneur en matières volatiles

- \résistivité électrique des particules de coke (agrégats)<smiles>[13CH3]</smiles>

Coke calciné<smiles>C1CCCC1</smiles>

- Broyage - Tamisage

- Mélange : coke calciné + Brai liant

- Mise en forme

1

Bloc:Coke + Brai

1

CUISSON $\left(1100^{\circ} \mathrm{C}\right)$

Buts :

- \teneur en produits volatils du brai

- cokéfaction des parties lourdes du brai

- amélioration des propriétés mécaniques et électriques

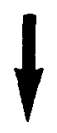

Electrodescartonées

Figure 1.1: Schéma général de la fabrication des électrodes carbonées dans l'industrie de l'aluminium. 


\section{I.3 Phénomènes physico-chimiques se produisant au cours de la calcination}

Au cours de la calcination du coke vert, on assiste à une série de phénomènes physico-chimiques complexes. A partir de $100^{\circ} \mathrm{C}$, la déshydratation du coke a lieu, suivie par la dévolatilisation débutant aux alentours de $450^{\circ} \mathrm{C}$. La dévolatilisation est le dégagement des matières volatiles de la masse solide de coke, suite à une êlévation de la température.

Les produits volatils se divisent en deux groupes: les produits dits condensables composés en grande partie d'hydrocarbures lourds et les produits non condensables dont les constituants majeurs sont le méthane et l'hydrogène. Les matières condensables se dégagent dans un domaine de températures comprises entre $450^{\circ} \mathrm{C}$ et $700^{\circ} \mathrm{C}$ approximativement. Les matières non condensables sont caractérisées par des zones de dégagement qui se chevauchent entre $600^{\circ} \mathrm{C}$ et $1200^{\circ} \mathrm{C}$.

Si la calcination est conduite dans un milieu oxydant (en présence d'air), les matières volatiles non condensables contiennent également les produits de la combustion, des volatils d'une part, et du coke d'autre part $\left(\mathrm{H}_{2} \mathrm{O}, \mathrm{CO}\right.$, $\left.\mathrm{CO}_{2}\right)$.

Le départ des matières volatiles du coke se traduit par une diminution de masse du coke (environ 10 à 12\%).

L'accroissement de l'épaisseur cristalline observée au cours du chauffage ainsi que le départ des volatils contribuent à la diminution de la résistivité électrique des particules de coke.

\section{I.4 . Travaux antérieurs}

De nombreux auteurs se sont penchés sur l'étude des phénomènes complexes se déroulant au cours du traitement thermique des produits carbonés, tant au niveau de la dégazéification qu'au niveau de la combustion en atmosphère oxydante. 
Au début des années 1970, au sein du Centre de recherche d'Alcan à Arvida, Belleau et Polak ont étudié la dévolatilisation du coke de pétrole en portant leur attention sur l'analyse des différents gaz émis au cours de la calcination [17,21]. La variation de poids est calculée par la différence entre les pesées avant et après le traitement thermique, sans se préoccuper de la cinétique proprement dite.

En ce qui concerne l'aspect cinétique, Wallouch et al. proposent un modèle basé sur l'analyse thermogravimétrique non isotherme, dans lequel la fixation de l'ordre de la réaction permet de déduire les autres paramètres cinétiques par la méthode différentielle [19,3,6]. En 1975, Anthony et Howard proposent un modèle de dévolatilisation des charbons en milieu non participant, basé sur une distribution gaussienne des énergies d'activation des nombreuses réactions, toutes d'ordre 1 [18]. Beér utilisera le modèle d'Anthony dans ses études sur la dévolatilisation et combustion des cokes en lit fluidisé (1985) [10,11].

En ce qui concerne l'aspect physique de la calcination, Rhedey a réalisé de nombreuses études au sujet des modifications structurales se produisant au cours de la pyrolyse du coke de pétrole, sous différentes conditions de température, pression, vitesse de chauffage, etc. $[23,25,26]$.

Parmi les études menées sur la combustion proprement dite des charbons, les travaux de Smith, au début des années 1980, constituent une excellente référence à la fois théorique et expérimentale $[7,14,15,20]$. Smith et Field, dans leurs êtudes, décrivent les différents régimes de combustion possibles en fonction de la température et donnent une expression générale de la vitesse de combustion des produits carbonés [14,22]. Smith a également accordé une attention particulière aux modifications structurales, surtout en ce qui concerne la porosité, la densité et la taille des particules, au cours des traitements thermiques [20].

On peut constater que la plupart des études menées sur la dévolatilisation et la combustion des produits carbonés ont été réalisées sur des échantillons de petite taille $(<1 \mathrm{~g})$ en vue de réduire l'influence du gradient de température $[4,14,15,16]$. Ainsi, elles s'éloignent de la réalité industrielle où des volumes importants de coke sont calcinés dans les fours pour constituer la matière première nécessaire à l'êlaboration des 
électrodes. De plus, la combustion de la particule de coke en atmosphère oxydante a été étudiée de manière approfondie, mais indépendamment de la dévolatilisation préalable; le taux de consommation de la particule étant proche de $100 \%[4,8,10,11,15,18,20]$.

Récemment néanmoins, une étude a été entreprise par le groupe de recherche GRIPS de l'UQAC sur la cinétique de calcination du coke de pétrole en milieu neutre, avec des échantillons de grande taille (20g) [2]. Toutefois, la présence d'air dans les fours industriels de calcination, nécessite des connaissances sur le comportement du coke en atmosphère oxydante, afin de maitriser de manière plus efficace le procédé.

Le travail entrepris dans le cadre de ce mémoire répond donc à un réel besoin. L'objectif est de comparer le comportement cinétique et l'évolution structurale d'échantillons représentatifs de coke de pétrole (éliminant les effets de non-homogénéité), au cours de sa calcination sous atmosphères neutre et oxydante.

Une attention particulière est apportée à la combustion, même si celle-ci est seulement amorcée et n'atteint que quelques pourcents ( 1 à $2 \%$ ), à $1200^{\circ} \mathrm{C}$ en fin de calcination.

L'originalité de cette étude réside dans le fait qu'elle porte sur deux aspects fondamentaux de la calcination: dévolatilisation et combustion, jusqu'ici généralement étudiés de façon indépendante par de nombreux auteurs $[2,4,5,6,8,10,11,18,20]$. Toutefois, deux études relatent les deux phénomènes à savoir dévolatilisation et combustion subséquente: l'une par Hargrave sur de petits échantillons de coke de pétrole entre 500 et $1000^{\circ} \mathrm{C}$ [9], l'autre par Goetz sur différents charbons entre 900 et $1800^{\circ} \mathrm{C}$ [13]. Toutes deux utilisent la thermogravimétrie isotherme et la relation d'Arrhénius pour déterminer les paramètres cinétiques.

\section{I.5 Contribution du présent travail dans la recherche}

\section{I.5.1 Objectif général}

Le présent travail vise à mieux comprendre la cinétique de "dévolatilisation-combustion" du coke vert au cours de sa calcination. 
En présence d'air, le carbone brûle à haute température donnant du monoxyde de carbone et du dioxyde de carbone. Au cours du chauffage, on assiste à la combustion des produits volatils provenant de la dévolatilisation (goudron, méthane et hydrogène) qui, en présence d'air, réagissent en libérant une certaine quantité de chaleur (réactions exothermiques) et des produits de combustion. Une fois dégagés de la masse solide de coke, les produits volatils constituent un film protecteur autour de la particule de coke. Ce film rétrécit par combustion plus ou moins rapide au contact de l'oxygène du milieu; si bien que la particule ne commence à brûler qu'à très haute température, une fois que l'oxygène se trouve au contact de la particule et si toutefois le taux de chauffage est suffisamment faible $[7,13,14,22]$.

Comme on peut le constater, le passage du milieu neutre au milieu oxydant ne se fait pas sans peine, puisqu'au phénomène de dévolatilisation vient s'ajouter la combustion des volatils et du coke, dans un contexte déjà très complexe de cinétique hétérogène solide-gaz.

Pour atteindre nos objectifs, la combinaison de l'analyse thermogravimétrique et de l'analyse chromatographique des gaz recueillis constitue un moyen efficace. L'analyse thermogravimétrique permet de déterminer les différents paramètres reliés à la cinétique de dégagement des volatils d'une part, et à la cinétique de combustion du résidu solide de coke d'autre part. L'analyse chromatographique fournit la composition des gaz émis lors de la pyrolyse.

\section{I.5.2 Quelques considérations cinétiques}

\section{I.5.2. A En ce qui concerne la dévolatilisation du coke vert}

La détermination des paramètres cinétiques à partir des résultats thermogravimétriques est basée sur le modèle de Wallouch et al. [3,6,19]. La dévolatilisation irréversible d'un solide au cours de sa pyrolyse est représentée par la réaction suivante:

$$
\text { s Sol -----> r Rés + v Vol }
$$


où Sol, Rés et Vol sont respectivement le solide original (coke vert), le résidu ou solide final (coke calciné) et les matières volatiles.

Le degré d'avancement de la réaction est exprimé par la formule suivante:

$$
\mathrm{dX} / \mathrm{dt}=\mathrm{k}(1-\mathrm{X})^{\mathrm{n}}
$$

où $\mathrm{X}$ représente la fraction des matières volatiles dégagées $(X=0$ au début de la pyrolyse et $\mathrm{X}=1$ à la fin) et $\mathrm{n}$, l'ordre de la réaction.

La constante cinétique $\mathbf{k}$ dépend de la température. En effet, la vitesse d'une réaction croît de manière spectaculaire avec la température selon la loi d'Arrhénius:

$$
k=A \exp ^{(-E / R T)}
$$

où A est le facteur pré-exponentiel en $\mathrm{s}^{-1}, E$ l'énergie d'activation en $\mathrm{J} / \mathrm{mol}$, $\mathrm{R}$ la constante des gaz parfaits $(8.317 \mathrm{~J} / \mathrm{mol} . \mathrm{K})$ et $\mathrm{T}$ la température absolue en $\mathrm{K}$. L'énergie d'activation représente l'énergie nécessaire à la formation du complexe activé (état de transition).

La vitesse de chauffage c étant constante lors des expëriences de pyrolyse, on peut écrire:

$$
\mathrm{dt}=\mathrm{dT} / \mathrm{c}
$$

En combinant les relations (I.3) et (I.4) avec l'équation (I.2), on obtient l'équation générale qui décrit la cinétique de la pyrolyse d'un solide dans un milieu non participant:

$$
\mathrm{dX} / \mathrm{dT}=(\mathrm{A} / \mathrm{c}) \exp ^{(-\mathrm{E} / \mathrm{RT})}(1-\mathrm{X})^{\mathrm{n}}
$$

Les paramètres cinétiques $\mathrm{A}, \mathrm{E}$ et $\mathrm{n}$ sont déterminés graphiquement (méthode différentielle). 


\section{I.5.2.B En ce qui concerne la combustion des volatils et du résidu de coke}

Il existe une différence fondamentale entre la réaction de combustion homogène des volatils en phase gazeuse, et la réaction subséquente de combustion hétérogène solide-gaz entre le résidu carboné et l'oxygène de l'air. La réaction en phase gazeuse débute dès que les volatils entrent en contact avec l'oxygène comburant; cette réaction est très rapide, comparativement à la réaction plus lente de combustion du coke solide. En effet, pour que la combustion du coke ait lieu, il faut que l'oxygène diffuse à travers les produits et les volatils pour atteindre la surface réactive solide; à ce moment là seulement, la réaction chimique se produit $[7,13,22]$.

Dans le processus de combustion des volatils, il y a deux étapes essentielles: une première étape pendant laquelle les volatils sont mélangés à l'oxygène et une deuxième étape pendant laquelle la réaction chimique a lieu [22,27]. L'étape limitante est soit la faculté que possèdent les volatils à se mélanger à l'oxygène de l'air par diffusion moléculaire, si la réaction chimique est rapide; soit la réaction chimique elle-même, dans le cas où le mélange intime se fait rapidement.

Les volatils, en se dégageant de la particule de coke, forment une protection autour de celle-ci, de telle sorte que l'oxygène comburant réagit avec les volatils mais ne peut atteindre la surface réactive solide de la particule, et ce aussi longtemps que le processus de dévolatilisation engendre une couche protectrice suffisamment épaisse pour éviter le contact.

Dans le processus de combustion de la particule poreuse de coke, il y a trois étapes essentielles: une première étape pendant laquelle l'oxygène diffuse à travers les gaz environnants pour atteindre la surface de la particule, une deuxième étape pendant laquelle l'oxygène diffuse plus ou moins profondément dans la structure interne poreuse de la particule et une troisième étape au cours de laquelle la réaction chimique a lieu $[22,27]$.

Ces trois étapes jouent un rôle important dans la détermination de la vitesse de combustion de la particule; et, suivant la température, le processus est limité par l'une, l'autre ou la combinaison de ces étapes. 
A basse température, la combustion est contrôlée par la réaction chimique, l'énergie d'activation apparente est égale à l'énergie réelle et il n'y a pas de gradient de concentration dans le solide; le gaz peut atteindre tous les sites actifs internes de la particule (Régime I).

A température moyenne, la combustion est contrôlée par la réaction chimique et la diffusion interne dans la particule; le gaz atteint la surface mais sa concentration diminue progressivement à l'intérieur de la particule. L'énergie d'activation apparente vaut la moitié de l'énergie réelle, l'ordre apparent de la réaction (n) est relié à l'ordre réel (m) par la relation: $\mathrm{n}=(\mathrm{m}+1) / 2$ (Régime II).

A haute température, la combustion est contrôlée par la diffusion externe dans les gaz environnants, la concentration du réactant est très faible à la surface de la particule et seule la diffusion de l'oxygène peut contribuer à accroître cette concentration (Régime III).

L'expression générale de la vitesse de combustion de la particule solide fait apparaître deux résistances: une première résistance " $1 / k_{\text {diff }}$ pour la diffusion externe des gaz et une deuxième résistance " $1 / \mathrm{k}_{\mathrm{s}}$ " qui englobe la diffusion interne et la réaction chimique. La vitesse de combustion q en [g/s] prend la forme suivante (pour un ordre de réaction unitaire) [14,22]:

$$
\mathrm{q}=\mathrm{pg}_{\mathrm{g}} /\left(1 / \mathrm{k}_{\text {diff }}+1 / \mathrm{k}_{\mathrm{s}}\right)
$$

où $\mathrm{pg}$ est la pression partielle en oxygène en atm, $\mathbf{k}_{\text {diff }}$ le coefficient de vitesse en diffusion externe et $k_{s}$ le coefficient de vitesse en réaction chimique et diffusion interne, en $\mathrm{g} / \mathrm{s}$.atm (pour un ordre de réaction unitaire). Le coefficient $\mathrm{k}_{\mathbf{s}}$ est relié à la température par la loi d'Arrhénius. Il est rapporté dans la littérature que la diffusion prend le contrôle à haute température pour de grosses particules; alors que la réaction chimique prend le contrôle pour de petites particules à basse température [22]. Dans notre contexte de travail (température moyenne, grosses particules) la combustion du résidu solide de coke après dévolatilisation se déroule en admettant deux étapes limitantes: la réaction chimique et la diffusion interne dans les pores. Cette affirmation est confirmée à l'examen de la structure du coke calciné: si le diamètre de la particule diminue au cours de la combustion en admettant une densité plus ou moins constante, la combustion se fait bien en régime II $[14,15,20,22]$. 


\section{I.5.3 Objectifs spécifiques}

Les objectifs spécifiques de la recherche sont les suivants:

1- L'influence des conditions de travail sur la cinétique de "dévolatilisation" du coke vert. Les paramètres variables sont la qualité du coke vert (principalement l'indice des matières volatiles), la vitesse de chauffage et la nature du milieu (neutre ou oxydant).

2- L'évolution structurale du coke (porosité, densité et épaisseur cristalline) au cours du traitement thermique. L'effet de la vitesse de chauffage, de la température et du palier de température sur la structure du coke calciné, en milieux neutre et oxydant. L'effet du pourcentage d'oxygène dans le milieu sur les propriétés du coke calciné.

3- La détermination des paramètres cinétiques globaux de la "dévolatilisation" du coke vert en atmosphères oxydante et neutre. Par paramètres cinétiques, on entend les paramètres qui permettent de définir complètement la cinétique du phénomène, c'est-à-dire l'ordre de la réaction $\mathrm{n}$, l'énergie d'activation $\mathrm{E}$ et la constante cinétique $\mathrm{k}$.

4- Enfin, la meilleure compréhension des mécanismes réactionnels complexes liés à la "dévolatilisation-combustion" du coke vert en atmosphère oxydante.

A cette fin, un dispositif thermogravimétrique a été mis au point. Des échantillons de coke vert $(10 \mathrm{~g})$ sont soumis à la pyrolyse à différents régimes de chauffage $\left(50\right.$ à $\left.150^{\circ} \mathrm{C} / \mathrm{min}\right)$. La perte de poids et la concentration des gaz sont enregistrées tout au long de la montée en température. 


\section{CHAPITRE II}

\section{TECHNIgUES D'ANALYSE ET MONTAGE EXPERIMENTAL}

\section{IIIIntroduction}

Le présent chapitre décrit les techniques d'analyse et donne une description détaillée du montage expérimental. Soulignons qu'une attention particulière a été portée à la réalisation d'un creuset qui se prête bien pour l'étude en milieu oxydant. Le contexte de cinétique hétérogène solide-gaz ainsi que le milieu oxydant "agressif" sont deux aspects directeurs dans la mise au point du montage et le choix du matériel expérimental.

\section{II.2 Techniques d'analyse}

Dans bien des études de cinétique chimique, c'est la vitesse de réaction qui est exprimée en fonction de différents paramètres mesurables, au moyen d'une équation différentielle déterminée; la loi d'évolution du système est alors déduite de l'intégration de cette équation et des conditions aux limites imposées par l'expérience.

La thermogravimétrie est la démarche inverse qui consiste, à partír des fractions de gaz recueillies et de la perte de poids enregistrée de manière continue, à définir l'évolution cinétique du phénomène étudié, en l'occurrence ici la dévolatilisation du coke vert lors de sa calcination.

La thermogravimétrie est l'association de la pesée continue de substances participant à une réaction, avec une variation linéaire de la température en fonction du temps. D'une manière générale, pour qu'un système soit justiciable de la thermogravimétrie, il faut que les substances soumises à la pesée subissent une variation de masse traduisant l'évolution du système. La dévolatilisation du coke vert au cours de sa calcination est un 
système qui répond à cette condition. En effet, au cours du chauffage jusqu'à $1200^{\circ} \mathrm{C}$, le départ des matières volatiles se traduit par une diminution de masse du coke. La thermogravimétrie est donc la technique appropriée pour étudier l'évolution du système dans le temps ou sa cinétique.

L'analyse des gaz prélevés à chaque température repère, au cours de la calcination, est réalisée à l'aide de la chromatographie en phase gazeuse. Les pics constituants les résultats chromatographiques ou "chromatogrammes" permettent d'identifier (temps de rétention) et de quantifier (aire sous le pic) les différents gaz émis. Une calibration est au préalable réalisée sur base d'un mélange étalon de gaz dont les concentrations sont connues exactement. Les caractéristiques du matériel chromatographique sont données dans le tableau 2.1 pour la colonne à tamis moléculaire et dans le tableau 2.2 pour la colonne capillaire. La colonne à tamis moléculaire permet la détection du méthane et de l'hydrogène; la colonne capillaire permet la détection des monoxyde et dioxyde de carbone.

Tableau 2.1: $\quad$ Conditions d'analyse en chromatographie gazeuse pour l'hydrogène et le méthane.

\begin{tabular}{|lll|}
\hline Chromatographe & $:$ & VARIAN 3400 \\
\hline Détecteur & $:$ & TCD (conductivité thermique) \\
\hline Colonne & $:$ & Tamis moléculaire 13X \\
\hline Conditions: & & \\
\hline & & $80^{\circ} \mathrm{C}$ \\
Temp. injecteur & $\vdots$ & $120^{\circ} \mathrm{C}$ \\
Temp. détecteur & $\vdots$ & $190^{\circ} \mathrm{C}$ \\
Temp. filament & $0.5 \mathrm{~mL}$ \\
Volume d'injection & $\vdots$ & Argon \\
Gaz porteur & $\vdots$ & $20 \mathrm{~mL} /$ min \\
Débit gaz porteur & $\vdots$ & Injection manuelle (seringue) \\
\hline
\end{tabular}


Tableau 2.2: $\quad$ Conditions d'analyse en chromatographie gazeuse pour les monoxyde et dioxyde de carbone.

\begin{tabular}{|lll|}
\hline Chromatographe & $:$ & VARIAN 3400 \\
\hline Détecteur & $:$ & TCD (conductivité thermique) \\
\hline Colonne & $:$ & Carbo plot 007 (capillaire) \\
\hline Conditions: & & \\
Temp. injecteur & $\vdots$ & $60^{\circ} \mathrm{C}$ \\
Temp. colonne & $\vdots$ & $60^{\circ} \mathrm{C}$ \\
Temp. détecteur & $\vdots$ & $200^{\circ} \mathrm{C}$ \\
Volume d'injection & $\vdots$ & $0.1 \mathrm{~mL}$ \\
Gaz porteur & Argon \\
Débit gaz porteur & $:$ & $20 \mathrm{~mL} / \min$ (colonne) \\
Injecteur & $:$ & $4.6 \mathrm{~mL} / \mathrm{min}$ (total) \\
& \\
\hline
\end{tabular}

\section{II.3 Montage expérimental}

\section{II.3.1 Description du montage}

Le montage expérimental peut être divisé en trois parties:

- l'analyse thermogravimétrique;

- l'analyse chromatographique;

- et l'acquisition des données.

Un schéma général du montage expérimental est donné à la figure 2.1.

La pyrolyse du coke vert est effectuée dans un four à induction vertical $(5 \mathrm{~kW})$, réalisé dans un tube de quartz dans lequel suspend le creuset destiné à recevoir l'échantillon. Le réacteur transparent présente l'avantage de visualiser le phénomène de dévolatilisation-combustion du coke tout au long de sa calcination. Le chauffage par induction permet de chauffer l'échantillon directement dans sa masse avec des vitesses de chauffage élevées (jusqu'à $150^{\circ} \mathrm{C}$ par minute), de manière à se rapprocher le plus possible des conditions qui prévalent dans les fours de calcination 
industriels. Un isolant entoure le creuset de sorte à réduire les pertes de chaleur vers l'ambiance au voisinage du creuset. Un courant de gaz préchauffé (azote ou azote+air) est envoyé dans le four sous une pression constante de une atmosphère.

Le creuset est suspendu à une balance de type METTLER 2000 qui mesure de façon continue la perte de poids de l'échantillon. La température est mesurée en continu à partir de $430^{\circ} \mathrm{C}$ à l'aide d'un pyromètre à infrarouge de type OMEGA OS1200, connecté à un contrôleur de température de type MICRISTAR et au générateur de hautes fréquences $(450 \mathrm{kHz})$, afin d'obtenir une montée linéaire en température. Durant la pyrolyse, les mesures de poids et de températures sont enregistrées à intervalle régulier (la seconde) par un système d'acquisition de données LABTECH NOTEBOOK, donnant ainsi la variation du poids en fonction de la température.

Une sonde d'échantillonnage placée dans la partie supérieure du four récolte les échantillons de gaz émis. Deux types de sondes ont été testées: la première présentant un plateau perforé sur la base inférieure de la tige; la seconde perforée sur toute la hauteur de la tige (figure 2.2). Une expérience préliminaire dévoile des résultats très similaires quant à la concentration des gaz recueillis par la sonde, pour les deux types de sondes; ce qui prouve que le mélange gazeux prélevé est bien représentatif du milieu réel, homogène dans le réacteur.

Les gaz prélevés à l'aide d'une seringue sont injectés dans le chromatographe pour l'analyse. L'analyse chromatographique ne porte que sur les gaz non condensables. L'échantillon de gaz est débarrassé de toute trace d'humidité et de goudron par passage dans un condenseur et des filtres de résine successivement, avant d'être envoyé au chromatographe. 


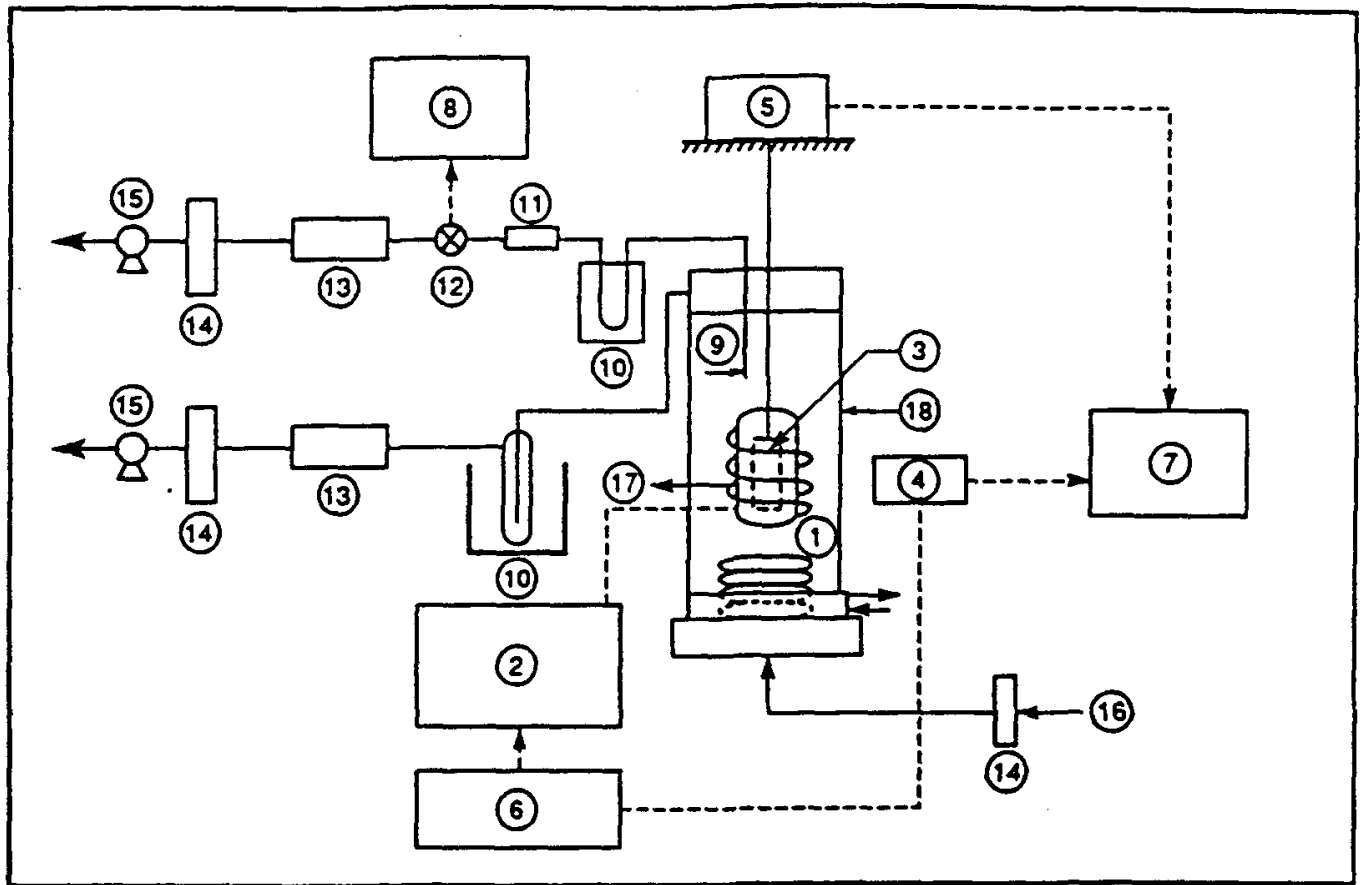

1) Four a induction

2) Générateur

3) Echanttllon

4) Pyromètre a infra-rouge

5) Balance

6) Controleur de température

7) Ordinateur

8) Chromatographe

9) Sonde d'échantillonnage gazeux
10) Condenseur

11) Filtres

12) Prise échantillon gazeux

13) Filtres

14) Rotamètre

15) Pompe aspirante

16) Gaz: azote et air

17) Isolant

18) Tube de quartz

Figure 2.1: Montage expérimental. 


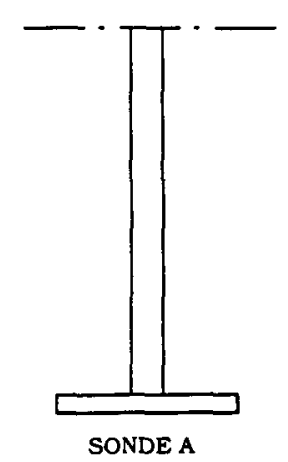

SONDE A

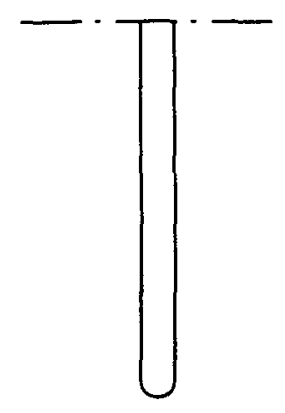

SONDE B

Figure 2.2: Types de sondes d'échantillonnage.

\section{II.3.2 Conditions expérimentales}

Durant les expériences de calcination du coke vert, la température augmente linéairement en fonction du temps selon trois taux de chauffage couvrant la gamme des taux de chauffage observês dans les fours industriels de calcination: $150^{\circ} \mathrm{C} / \mathrm{min}, 100^{\circ} \mathrm{C} / \mathrm{min}$ et $50^{\circ} \mathrm{C} / \mathrm{min}$. Les trois taux de chauffage sont présentés à la figure 2.3.

Quatre types de cokes verts ont fait l'objet de l'étude: les cokes TEXACO, RTW, YPF et CONOCO. Les caractéristiques des cokes sont données dans le tableau 2.3.

Les conditions expérimentales de calcination sont reprises dans le tableau 2.4. Les expériences ont été répétées plusieurs fois afin de juger de la reproductibilité des résultats. Ainsi, on peut affirmer que dans tous les cas une bonne reproductibilité est obtenue aussi bien en ce qui concerne les thermogrammes qu'en ce qui concerne l'analyse des gaz. 


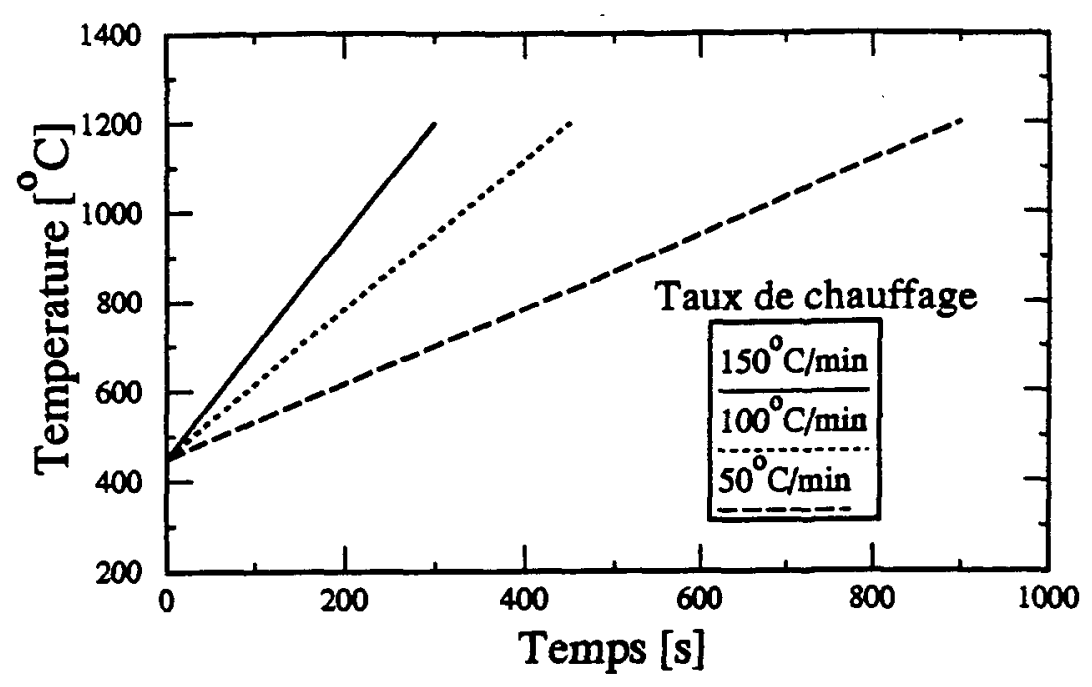

Figure 2.3: Taux de chauffage au cours des expériences de calcination.

Tableau 2.3: $\quad$ Propriétés des cokes verts.

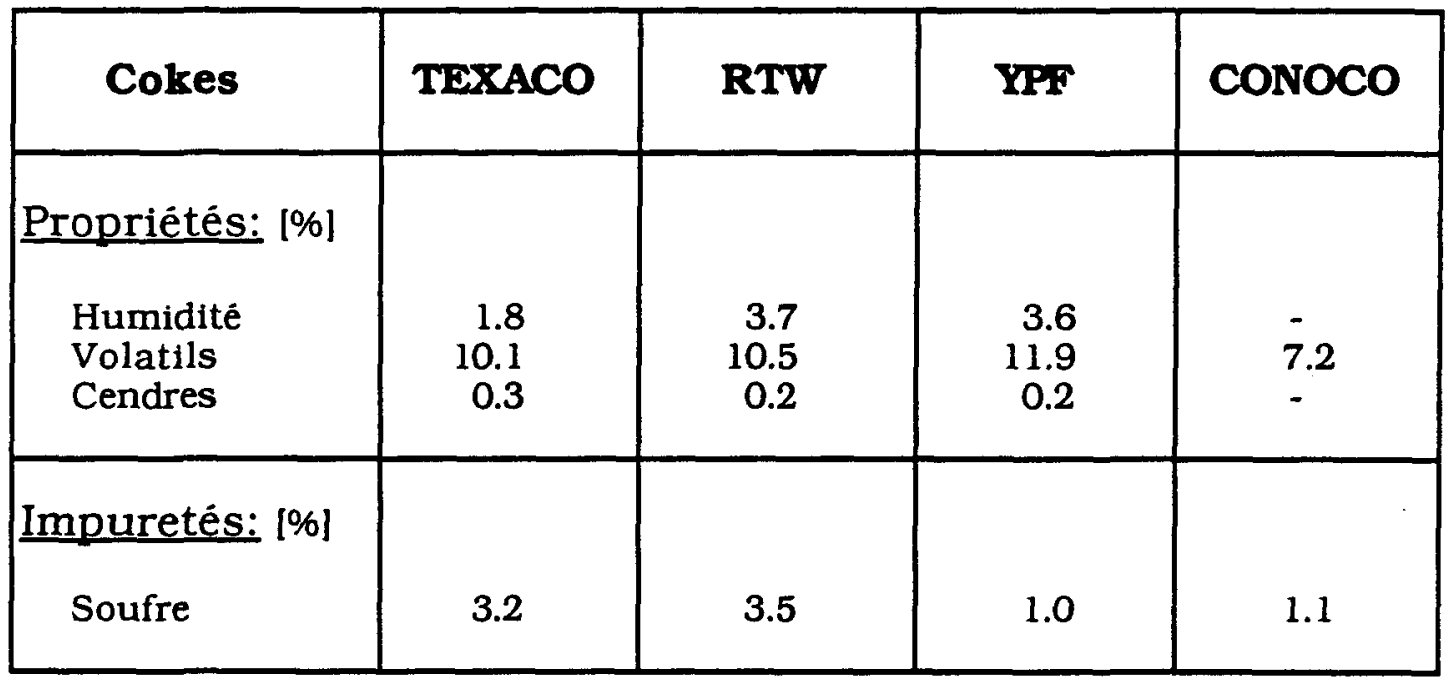


Le gaz ambiant (azote ou azote+air) est préchauffé dans la partie inférieure du tube de quartz et envoyé sous un débit de 10.6 litres/minute dans l'enceinte, de manière à éviter toute entrée d'air extérieur dans le tube.

Des expériences préliminaires ont mis en évidence l'existence d'un délai de temps (ou de température) entre le moment où les gaz sont aspirés par la sonde d'êchantillonnage et le moment où ils sont prélevés à l'aide de la seringue pour l'analyse. En effet, les gaz sont débarrassés de toute trace d'humidité et de goudron avant d'être envoyés au chromatographe pour l'analyse. On constate expérimentalement que l'augmentation du débit d'aspiration de $98 \mathrm{~mL} / \mathrm{min}$ à $313 \mathrm{~mL} / \mathrm{min}$ déplace les courbes de concentration des gaz de 1 minute vers la gauche, ou $100^{\circ} \mathrm{C}$ et $150^{\circ} \mathrm{C}$ pour les deux taux de chauffage respectifs de $100^{\circ} \mathrm{C} / \mathrm{min}$ et $150^{\circ} \mathrm{C} / \mathrm{min}$. Au delà de $313 \mathrm{~mL} / \mathrm{min}$, le dẻbit d'aspiration n'influence plus les résultats. Par contre, pour le taux de chauffage le plus faible de $50^{\circ} \mathrm{C} / \mathrm{min}$, l'augmentation du débit d'aspiration des gaz n'a aucune influence sur la concentration des gaz recueillis.

Par conséquent, le débit d'aspiration des gaz est fixé à $313 \mathrm{~mL} / \mathrm{min}$ pour les trois taux de chauffage, afin de supprimer le délai dû au parcours des gaz dans la ligne de préparation pour le chromatographe. En fait, plus le taux de chauffage est élevé, plus vite doivent être aspirés les gaz pour éviter tout problème de délai. 
Tableau 2.4: Conditions expérimentales de calcination en milieux neutre et oxydant.

\begin{tabular}{|c|c|c|c|c|}
\hline Cokes & $\begin{array}{l}\text { Nature du } \\
\text { milieu }\end{array}$ & $\begin{array}{l}\text { Taux de } \\
\text { chauffage } \\
{\left[{ }^{\circ} \mathrm{C} / \mathrm{min}\right]}\end{array}$ & $\begin{array}{c}\text { Nombre } \\
\text { d'expériences }\end{array}$ & $\begin{array}{l}\text { Type de } \\
\text { creuset }\end{array}$ \\
\hline TEXACO & $\begin{array}{l}100 \% \mathrm{~N}_{2} \\
97 \% \mathrm{~N}_{2}, 3 \% \mathrm{O}_{2} \\
95 \% \mathrm{~N}_{2}, 5 \% \mathrm{O}_{2} \\
93 \% \mathrm{~N}_{2}, 7 \% \mathrm{O}_{2} \\
90 \% \mathrm{~N}_{2}, 10 \% \mathrm{O}_{2}\end{array}$ & $\begin{array}{l}150^{(* * *)} \\
100 \\
50 \\
150^{(* * *)} \\
150^{(* * *)} \\
100^{(* 0} \\
50 \\
150^{(*)}\left(^{(* * *)}\right. \\
150^{(*)}\left(^{(* *)}\right)\end{array}$ & $\begin{array}{l}5 \\
4 \\
4 \\
3 \\
\\
\\
5 \\
5 \\
4 \\
3\end{array}$ & $\begin{array}{l}\text { ouvert, fermé } \\
\text { ouvert, fermé } \\
\text { ouvert, fermé } \\
\text { ouvert } \\
\text { ouvert } \\
\text { ouvert } \\
\text { ouvert } \\
\text { ouvert } \\
\text { ouvert }\end{array}$ \\
\hline RTW & $\begin{array}{l}100 \% \mathrm{~N}_{2} \\
95 \% \mathrm{~N}_{2}, 5 \% \mathrm{O}_{2}\end{array}$ & $\begin{array}{r}150^{(* * *)} \\
100^{(* * *)} \\
50^{(* * *)} \\
150^{(* * *)} \\
100^{(* * *)} \\
50^{(* * *)}\end{array}$ & $\begin{array}{l}3 \\
3 \\
3 \\
\\
5 \\
6 \\
4\end{array}$ & $\begin{array}{l}\text { ouvert } \\
\text { ouvert } \\
\text { ouvert } \\
\text { ouvert } \\
\text { ouvert } \\
\text { ouvert }\end{array}$ \\
\hline YPF & $\begin{array}{l}100 \% \mathrm{~N}_{2} \\
95 \% \mathrm{~N}_{2}, 5 \% \mathrm{O}_{2}\end{array}$ & $\begin{array}{r}150 \\
100 \\
50 \\
150 \\
100 \\
50\end{array}$ & $\begin{array}{l}2 \\
2 \\
2 \\
\\
5 \\
4 \\
3\end{array}$ & $\begin{array}{l}\text { ouvert } \\
\text { ouvert } \\
\text { ouvert } \\
\text { ouvert } \\
\text { ouvert } \\
\text { ouvert }\end{array}$ \\
\hline CONOCO & $\begin{array}{l}100 \% \mathrm{~N}_{2} \\
95 \% \mathrm{~N}_{2}, 5 \% \mathrm{O}_{2}\end{array}$ & $\begin{array}{c}150^{(* *)} \\
100 \\
50 \\
150^{(* *)} \\
100 \\
50\end{array}$ & $\begin{array}{l}4 \\
2 \\
2 \\
\\
6 \\
5 \\
4\end{array}$ & $\begin{array}{l}\text { ouvert } \\
\text { ouvert } \\
\text { ouvert } \\
\text { ouvert } \\
\text { ouvert } \\
\text { ouvert }\end{array}$ \\
\hline
\end{tabular}


(*) : Les expériences sont limitées à $150^{\circ} \mathrm{C} / \mathrm{min}$ à cause de la détérioration du creuset et l'explosion mineure se produisant à faible taux de chauffage (50 et $100^{\circ} \mathrm{C} / \mathrm{min}$ ) et $\%$ élevé en oxygène (à partir de $7 \%$ ).

$(* *) \quad$ : Les essais au cours desquels les échantillons de coke sont prélevés pour l'analyse structurale ultérieure. Des paliers de 15 et $30 \mathrm{~min}$ à $1200^{\circ} \mathrm{C}$ ont êté réalisés. La durée maximale du palier est fixée à 30 min à cause de la détérioration des attaches supportant le creuset audelà de $30 \mathrm{~min}$ à haute température et en présence d'air.

(***) : Les essais au cours desquels les échantillons de coke sont prélevés pour l'analyse structurale ultérieure. Les échantillons sont récoltés à différentes températures au cours du chauffage.

Creuset de type "ouvert": Creuset mis au point dans le cadre de cette présente étude. Creuset de type "fermê": Creuset utilisé dans une étude antérieure [2,3].

\section{II.4 Conception originale du creuset}

\section{II.4.1 Guant au matériau}

Le choix d'un matériau adéquat pour la réalisation du creuset ainsi que la conception même de la forme du creuset ont fait l'objet d'une étude préliminaire. Ces deux aspects, qualitatif et conceptionnel du creuset méritent toute notre attention car ils conditionnent le bon déroulement des expẻriences et la validité des résultats.

Le graphite est un matériau réfractaire aux multiples propriétés qui lui assurent de nombreuses applications à haute température, et notamment dans la fabrication des creusets pour les fours à induction. Parmi les qualités du graphite, citons par exemple sa remarquable tenue aux chocs thermiques, son usinage facile, sa bonne conductibilité électrique et thermique. Toutefois, à partir de $650^{\circ} \mathrm{C}$, en présence de l'oxygène de l'air, le graphite s'oxyde en se consommant, de telle sorte que son utilisation en temps que tel ne peut être envisagée dans nos conditions de travail, à cause des phénomènes de combustion occasionnant la détérioration rapide et inévitable du creuset.

Plusieurs alternatives ont été envisagées pour palier ces risques de combustion du creuset en présence d'oxygène. Parmi celles-ci citons par exemple l'emploi d'un matériau non combustible tel que le platine. Ce 
dernier offre une bonne résistance à l'oxydation; toutefois, il présente l'inconvénient d'être fragile à haute température et coûteux. Une autre alternative serait d'utiliser les matériaux céramiques réfractaires de type alumine ou zircone; toutefois, à haute température, il peut naitre dans ces matériaux caractérisés par une faible résistance aux chocs thermiques, des contraintes capables de briser le creuset en raison des variations locales de températures occasionnées par le chauffage ou le refroidissement. De plus, la conductivité électrique des céramiques ne permet pas d'utiliser ces matériaux dans le contexte d'un chauffage par induction.

Notre choix s'est alors porté sur le creuset en graphite auquel on fait subir au préalable un traitement de protection qui lui confère une bonne résistance en milieu oxydant. Ce traitement consiste en la réalisation d'un recouvrement superficiel du creuset par un enduit protecteur spécifique, à base de résine et de poudre céramique $\mathrm{TiB}_{2}$ (GRAPHI COAT 623). Cet enduit s'est révélé être très résistant en atmosphère oxydante. Compte tenu de la variêté des conditions expérimentales utilisées, il est difficile de quantifier de façon précise la durabilité de l'enduit. Cependant, à titre d'exemple, on peut mentionner que l'enduit a résisté 2 heures à $1200^{\circ} \mathrm{C}$ dans un milieu contenant $5 \%$ d'oxygène.

\section{II.4.2 Guant à la forme}

Dans un contexte de cinétique hétérogène solide-gaz, il est nécessaire d'avoir un contact entre les particules solides de coke et le gaz comburant (oxygène de l'air) car le processus de combustion du coke ne s'amorce que dans ces conditions. Afin d'assurer une surface réactionnelle solide-gaz maximale, la forme du creuset a dû être révisée plusieurs fois et améliorée.

Au cours des essais préliminaires sur un creuset de forme annulaire, on a pu remarquer la nécessité de prévoir une section de préchauffage des gaz dans la partie inférieure du creuset afin d'assurer un contact intime entre les gaz chauds et les particules de coke.

En milieu neutre, les expériences antérieures ont été réalisées sur des échantillons de coke vert de granulométrie fine $(0.42$ à $0.83 \mathrm{~mm})$ [2]. En 
milieu oxydant, on a pu constater que ce lit de particules fines offrait une résistance trop élevée au passage du courant gazeux, si bien que l'oxydation s'effectuait sur une très petite superficie en contact avec le gaz. L'emploi d'une granulométrie plus grosse $(1.65$ à $2.36 \mathrm{~mm})$ a permis de résoudre le problème en offrant une circulation plus facile des gaz.

Plusieurs conceptions ont été testées expérimentalement pour finalement en arriver à un creuset d'une conception tout à fait originale qui se révèle être bien adapté à ce type d'étude en milieu oxydant; une préoccupation importante étant de maintenir le coke à une température la plus uniforme possible au cours des expériences de calcination.

Le creuset comprend deux parties:

1 - Une partie inférieure destinée au préchauffage des gaz; constituée d'un cylindre plein de $4.44 \mathrm{~cm}$ de diamètre sur $4.5 \mathrm{~cm}$ de hauteur; ce cylindre est perforé circonférentiellement de 6 petits cylindres creux de $0.9 \mathrm{~cm}$ de diamètre, sur toute la hauteur du cylindre plein.

2- Une partie supérieure destinée à recevoir l'échantillon; constituée d'un cylindre plein de $4.44 \mathrm{~cm}$ de diamètre sur $4.5 \mathrm{~cm}$ de hauteur, dans lequel on aménage un anneau circonférentiel. Les parois de l'anneau sont perforées d'une multitude de trous permettant un contact intime entre les particules de coke et les gaz.

Un schéma du creuset est donné à la figure 2.4. Un couvercle perforé est déposé sur l'échantillon de manière à éviter l'échappement de particules au cours de l'expérience. Dans cet ouvrage, le creuset est qualifié de creuset de type "ouvert", par opposition au creuset de type "fermé" utilisé dans une étude antérieure en milieu neutre [2,3]. 


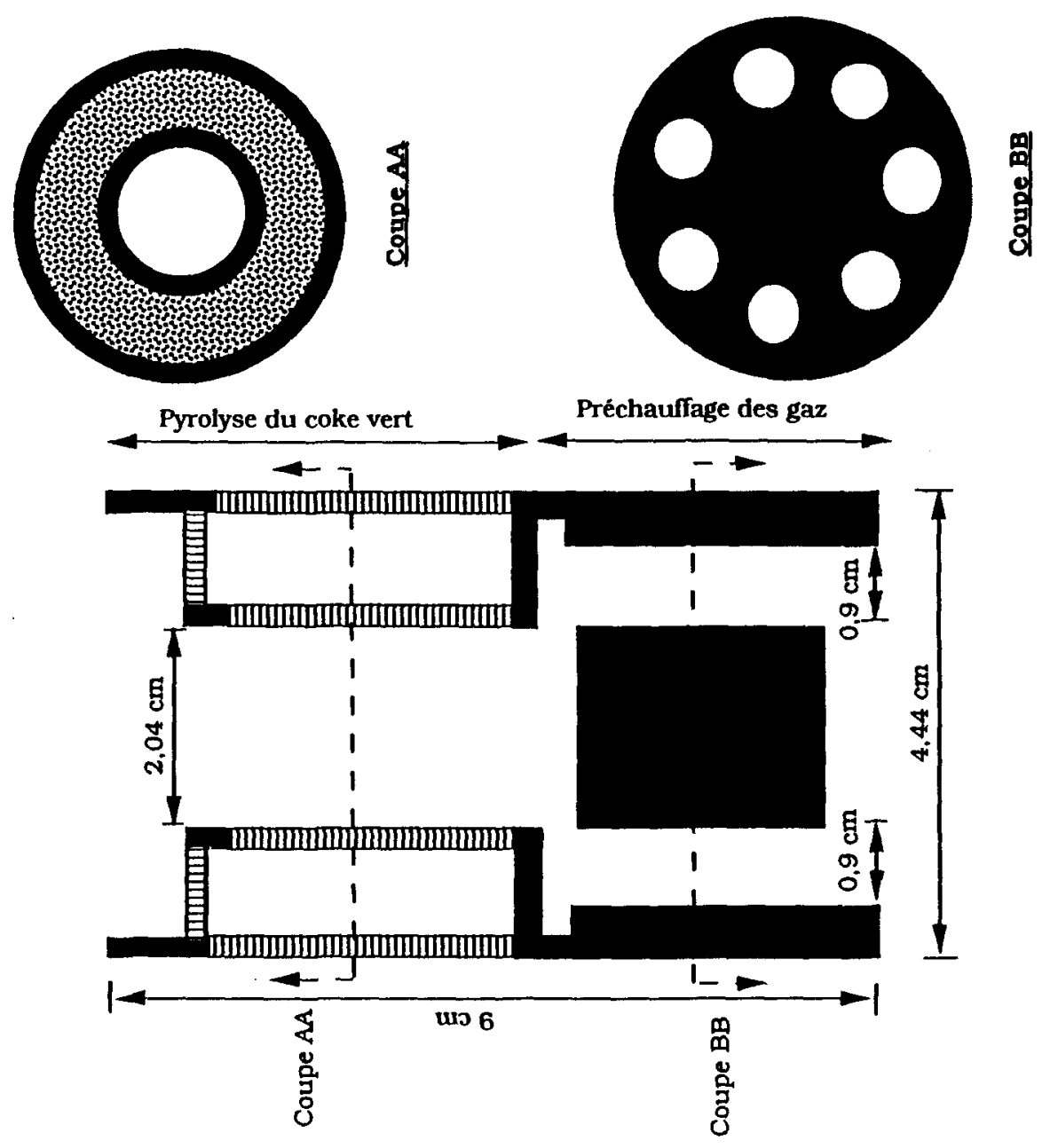

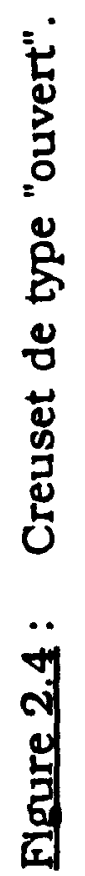




\section{Creuset de type "ouvert"}

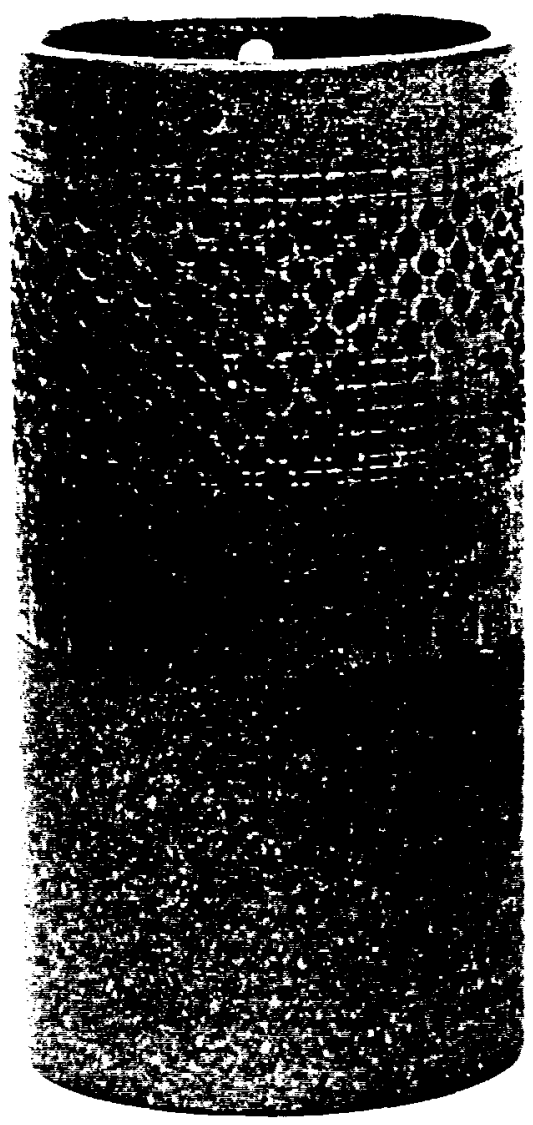

Prèchauffage des gaz

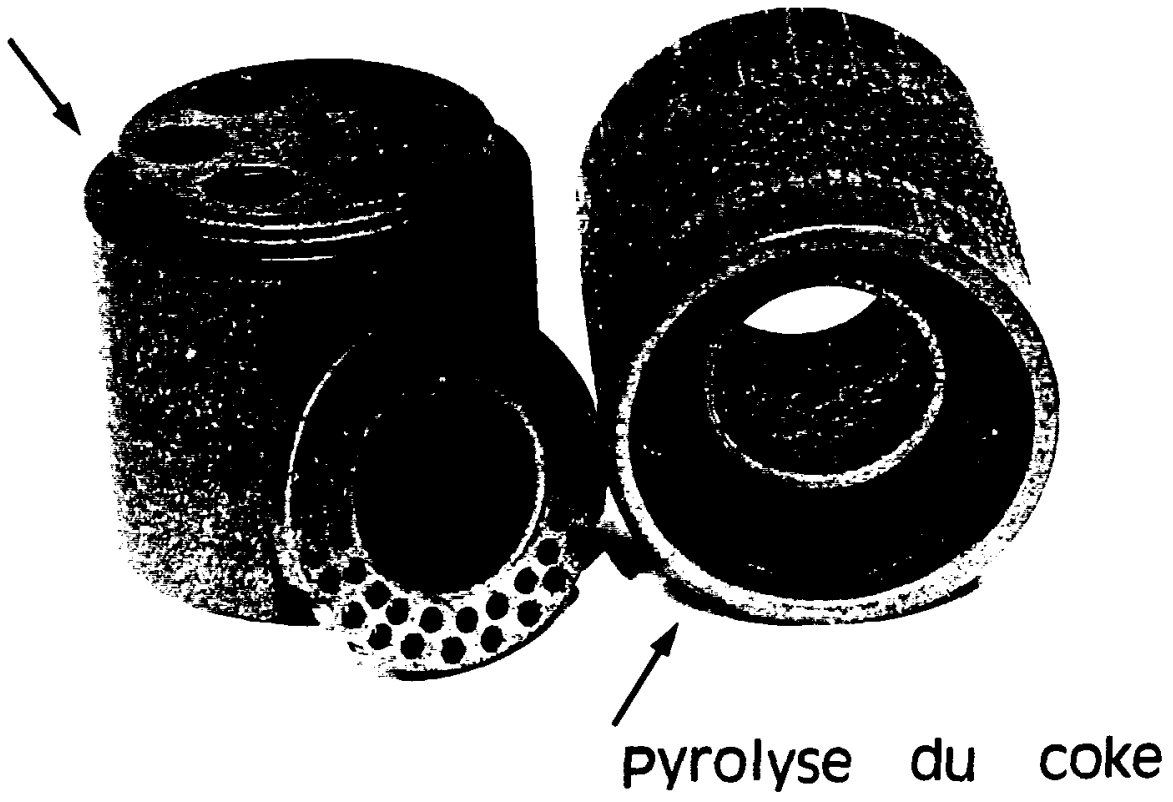

Figure 2.5 : Photo du creuset de type "ouvert". 


\section{CHAPITRE III}

\section{PRESENTATION DES THERMOGRAMMES ET DES CHROMATOGRAMMES}

\section{III.1 Thermogrammes}

\section{III.1.1 Correction à apporter aux résultats thermogravimétriques}

Les résultats thermogravimêtriques ou "thermogrammes" représentent l'évolution de la perte de poids de l'échantillon en fonction du temps ou de la température, au cours d'un cycle de chauffage de $450^{\circ} \mathrm{C}$ à $1200^{\circ} \mathrm{C}$.

Deux corrections sont apportées aux résultats thermogravimétriques: une première correction tient compte de la force induite par le champ magnétique; une seconde correction tient compte de la poussée des gaz chauds sur le creuset supportant l'échantillon. L'ensemble "creuset+échantillon" pèse environ 130 grammes.

\section{III.1.1.A Force induite par le champ magnétique}

L'existence d'une telle force a été mise en évidence lors d'essais préliminaires réalisés sur des échantillons de coke calciné, exempt de matières volatiles.

Rappelons que le chauffage de l'échantillon supporté par le creuset est réalisé dans un four à induction. On sait qu'un conducteur symétrique, de forme régulièrement uniforme, placé dans un champ magnétique uniforme produit par un courant traversant un solénoïde régulier, subit une force égale en amplitude et dirigée de bas en haut pour la partie supérieure du conducteur, et de haut en bas pour la partie inférieure. Ces deux forces $F_{1}$ et $F_{2}$ directement opposées et égales s'annulent et n'ont donc aucun effet sur le conducteur (voir figure 3.1). Rappelons utilement que cette force est 
maximale aux endroits où le gradient du champ magnétique est le plus élevé, c'est-à-dire aux extrémités de la bobine; et non au centre de la bobine où le champ est maximal.

Dans notre cas, le conducteur constitué par le creuset n'est pas symétrique si bien qu'il est impossible de trouver la position idéale du creuset dans le solénoïde qui permettrait d'annuler ces deux forces d'extrémité. La valeur de la force induite par le champ magnétique a été déterminée expérimentalement au cours d'un cycle de chauffage. C'est alors que l'on a pu constater qu'il existe une position idéale du creuset qui minimise cette force (à $10 \%$ de la perte de poids totale de l'échantillon). L'amplitude maximale de la force induite par le champ magnétique est observée en fin de cycle de chauffage (à $1200^{\circ} \mathrm{C}$ ), lorsque l'on arrête le gênérateur de hautes fréquences. La force induite par le champ magnétique est nulle ou négligeable au début du chauffage jusqu'à environ $450^{\circ} \mathrm{C}$; ensuite au fur et à mesure que l'on accroît la puissance du générateur pour assurer le chauffage, la force augmente linéairement jusqu'à $1200^{\circ} \mathrm{C}$ où elle atteint sa valeur maximale.

Ces constatations sont celles observées dans nos conditions expérimentales bien spécifiques. Le but de ce travail n'est pas d'expliquer plus en détail le phénomène électro-magnétique observé, mais de mettre en évidence les éléments perturbateurs de nos résultats afin d'y apporter une correction adéquate. Signalons quand même que les observations faites au cours de l'expérience préliminaire sont en accord avec la propriété diamagnétique du graphite qui est attiré dans la direction où l'intensité du champ magnétique est plus faible c'est-à-dire vers les extrémités du solénoïde (démontré à température ambiante). Nos conditions expérimentales nous imposant un chauffage jusqu'à $1200^{\circ} \mathrm{C}$, il est certain qu'à haute température de nombreux phénomènes, que nous ne tenterons pas d'expliquer ici dans le cadre de ce travail, s'ajoutent au phénomène déjà complexe de magnétisme. 


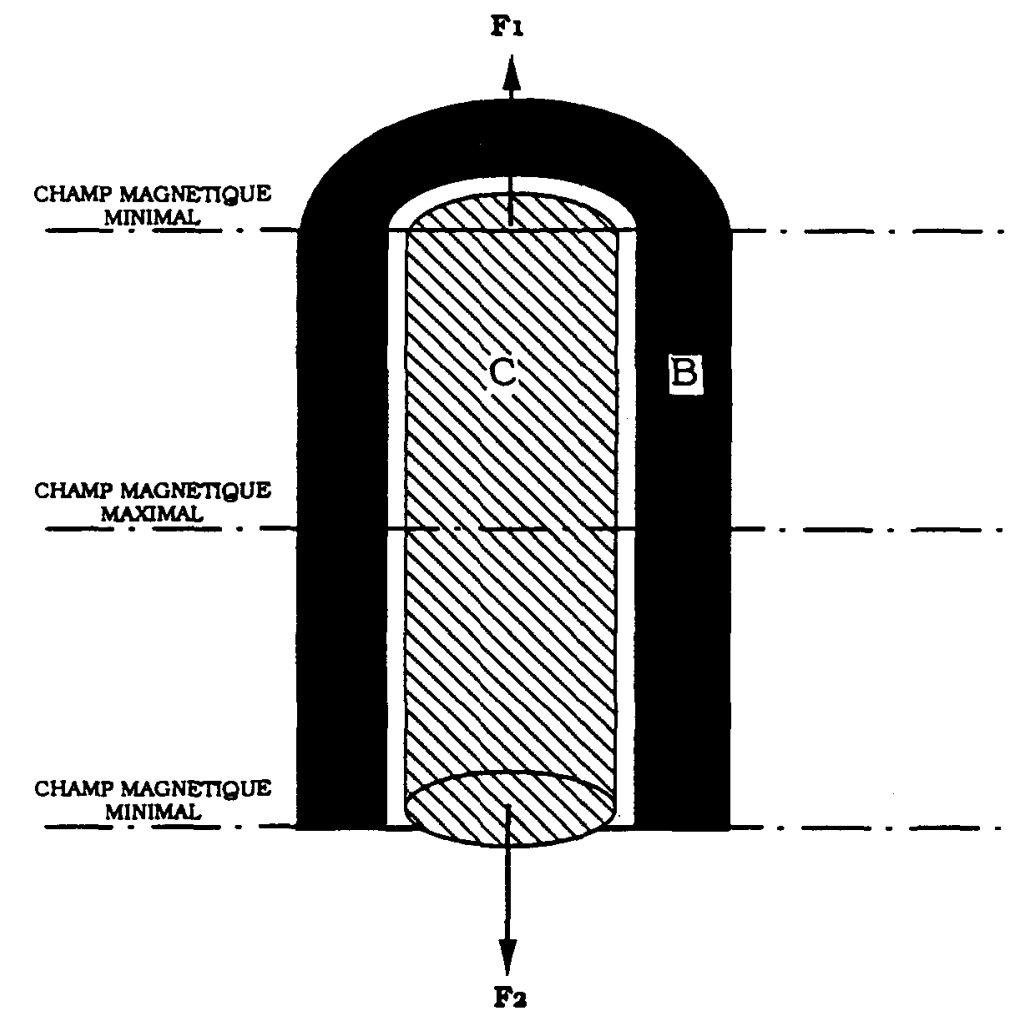

B : Bobine parcourue par courant

C : $\quad$ Conducteur diamagnétique au centre de la bobine

$F_{1}, F_{2} \quad$ : $\quad$ Forces dues au champ magnétique

Figure 3.1: Forces induites par un champ magnétique sur un conducteur symétrique (en coupe transversale). 


\section{III.1.1.B Poussée des gaz chauds}

Une deuxième correction tient compte de la poussée des gaz chauds qui agit sur le creuset supportant l'échantillon. Lorsqu'on chauffe un gaz, la masse volumique diminuant, la poussée d'Archimède diminue également; et l'ensemble "creuset+échantillon" se conduit comme s'il était plus lourd. Toutefois, au cours du chauffage, on observe expérimentalement une poussée des gaz chauds dirigée de bas en haut qui tend à soulever le creuset. Ceci signifie que l'accroissement de vitesse des gaz chauds l'emporte sur la diminution de la poussée d'Archimède lorsque la température augmente; ce qui engendre l'existence d'une poussée verticale de bas en haut.

L'amplitude de cette poussée des gaz chauds croît linéairement depuis la température ambiante où elle est nulle jusqu'à $1200^{\circ} \mathrm{C}$ où elle est maximale. A $1200^{\circ} \mathrm{C}$, elle vaut environ 4 à $5 \%$ de la perte de poids totale de l'échantillon. Cette poussée des gaz chauds doit être retranchée des résultats thermogravimétriques.

\section{III.1.2 Présentation des thermogrammes}

Tous les thermogrammes présentés dans cet ouvrage ont étê corrigés en considérant la variation linéaire de la force induite par le champ magnétique d'une part, et de la poussée des gaz chauds d'autre part. Ils représentent l'évolution de la perte de poids en fonction du temps ou de la température, au cours de la calcination du coke vert.

\section{III.2 Chromatogrammes}

\section{III.2.1 Correction à apporter aux résultats chromatographiques}

Les échantillons de gaz prélevés au cours de la calcination, font l'objet d'une analyse chromatographique. Au préalable, les résultats chromatographiques sont corrigés afin de tenir compte de l'air éventuellement infiltré dans la seringue au cours de la prise manuelle de 
l'échantillon gazeux. En milieu neutre, la quantitē d'air infiltrée est estimée par le volume d'oxygène présent dans l'échantillon gazeux. Par contre, en milieu oxydant, l'oxygène varie continuellement au cours du traitement thermique, on ne peut donc plus se baser sur l'oxygène pour estimer l'air infiltré dans la seringue.

\section{III.2.1.A En milieu neutre}

On réalise au préalable la calibration de l'air composé de $21 \%$ d'oxygène et de $79 \%$ d'azote; cette calibration permet la détermination de l'aire moyenne sous le pic relatif à l'oxygène de l'air:

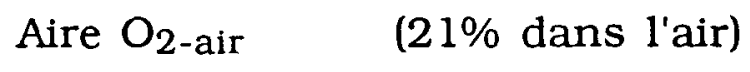

Au cours de la chromatographie des échantillons gazeux, le pourcentage d'oxygène infiltré lors de la prise est calculé comme suit:

$$
\% \mathrm{O}_{2 \text {-êch }}=21\left(\text { Aire } \mathrm{O}_{2} / \text { Aire } \mathrm{O}_{2 \text {-air }}\right)
$$

Le ratio de dilution est le rapport entre le volume de gaz prélevé et le volume total de gaz duquel on retranche le volume d'air:

$$
\begin{aligned}
& \% \text { airéch }=\left(\% \mathrm{O}_{2 \text {-êch }}\right)(100 / 21) \\
& \mathrm{RD}=\text { Volume totaléch } /(\text { Volume totaléch-Volume d'air }) \\
& \mathrm{RD}=1 /\left[\left(100-\left(\% \mathrm{O}_{2 \text {-êch }}(100 / 21)\right)\right) / 100\right]
\end{aligned}
$$

Après simplification de la relation (III.3), le ratio de dilution s'exprime selon la formule suivante:

$$
\mathrm{RD}=21 /\left(21-\% \mathrm{O}_{2 \text {-éch })}\right.
$$

Le ratio de dilution est toujours positif, ou ègal à l'unité s'il n'y a aucune trace d'air dans l'échantillon. 
L'aire corrigée, relative au gaz analysé prend la forme suivante:

$$
\text { Aire }(g a z)_{\text {corr }}=(R D) \text { Airegaz }
$$

\section{III.2.1.B En milieu oxydant}

Etant donné la variation continuelle du pourcentage d'oxygène au cours des expériences de calcination en présence d'air, aucune correction n'a été apportée aux résultats chromatographiques obtenus en milieu oxydant. Signalons cependant que le pourcentage d'air maximal obtenu dans les analyses chromatographiques réalisées en milieu neutre avoisine les quelques pourcents seulement (de l'ordre de 1 à 4\%); si bien que cet air infiltré au cours de l'échantillonnage manuel n'occasionne pas de correction majeure et que les résultats chromatographiques en milieu oxydant restent très fiables.

\section{III.2.2 Calcul de la concentration des gaz par la méthode des standards externes}

La calibration par la méthode des standards externes consiste à injecter un mélange étalon contenant les gaz d'intérêt dans des concentrations connues. Par exemple, en milieu neutre, le mélange étalon contient $\mathrm{x}$ ppm de $\mathrm{CH}_{4}$ et y ppm d'H $\mathrm{H}_{2}$; le reste étant l'azote. Le chromatogramme obtenu à partir du mélange étalon permet de calculer le facteur de réponse relatif à chaque composé d'intérêt de la manière suivante:

$$
F R_{i}=A_{i}^{\prime} / C_{i}^{\prime}
$$

où $\mathrm{A}_{\mathrm{i}}^{\prime}$ est l'aire sous le pic du composé $\mathrm{i}$ dans le mélange étalon et $\mathrm{C}_{\mathrm{i}}^{\prime}$ la concentration du composé i dans le mélange étalon exprimée en ppm (dans les mêmes unités que celles désirées pour l'analyse des gaz réels). 
Après la calibration, lors des injections de gaz, le calcul des concentrations de chaque composé s'effectue de la sorte:

$$
\mathrm{C}_{\mathrm{i}}=\mathrm{A}_{\mathrm{i}} / \mathrm{FR}_{\mathrm{i}}
$$

où $A_{i}$ est l'aire sous le pic du composé i de l'échantillon gazeux et $C_{i}$ la concentration du composé i dans l'échantillon exprimée en ppm.

La figure 3.2 donne un exemple de chromatogramme obtenu avec la colonne à tamis moléculaire (partie supérieure de la figure) et la colonne capillaire (partie infërieure de la figure).

a) Colonne à tamis moléculaire:

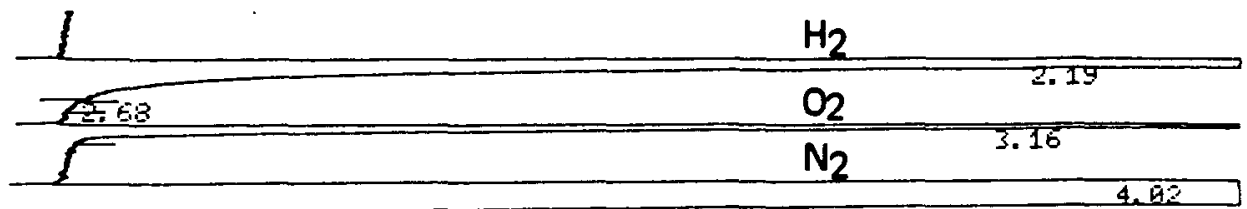

4. 67

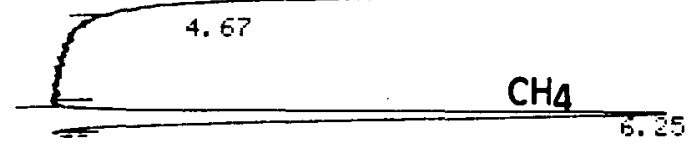

b) Colonne capillaire:

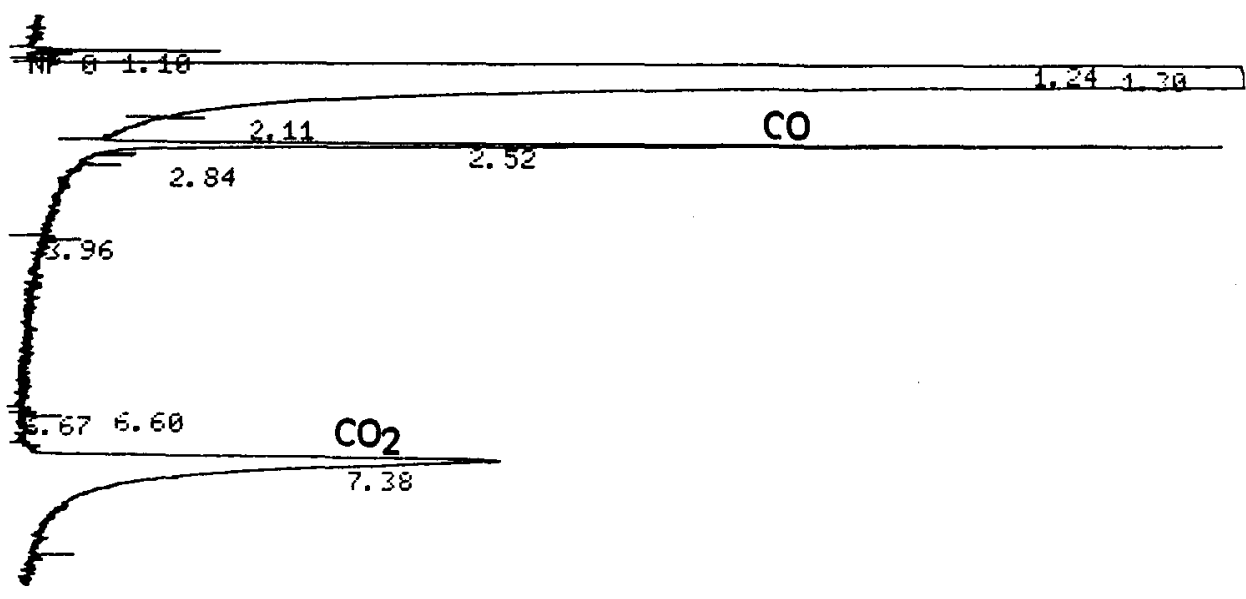

Figure 3.2: Exemple de chromatogrammes. 
CHAPITRE IV

VERIFICATION DU GRADIENT DE TEMPERATURE DANS LE CREUSET

\section{IV.1 Introduction}

Préalablement aux expériences proprement dites de calcination du coke vert, on a vérifié le gradient de température dans l'échantillon supporté par le creuset de type "ouvert". Les gradients de température radial et vertical dans l'échantillon de coke TEXACO ont été vérifiés pour les trois taux de chauffage utilisés dans nos expériences, en atmosphères neutre ( $100 \%$ azote) et oxydante ( $5 \%$ oxygène et $95 \%$ azote).

\section{IV.2 Quelques considérations sur le chauffage par induction}

Le chauffage du creuset en graphite enduit se fait par induction c'està-dire par l'intermédiaire de courants induits dans le creuset qui se dissipent sous forme de chaleur par effet Joule. On sait que dans un corps chauffé par induction, les courants induits décroissent exponentiellement de la périphérie vers le centre et tout se passe comme si le courant était localisé dans une épaisseur $d_{o}$ définie par la relation:

$$
\mathrm{d}_{\mathrm{o}}=\sqrt{\rho /(\mu . \mathrm{f})} / 2 \pi
$$

La profondeur qui caractérise le phénomène appelé "effet pelliculaire" est donc inversement proportionnelle à la racine carrée de la fréquence. Le gênérateur produit un courant alternatif de fréquence $\mathrm{f}(450 \mathrm{kHz}), \mu$ est la perméabilité magnétique et $\rho$ la résistivité électrique du graphite. Dans ces conditions, l'épaisseur des courants induits dans le creuset vaut $d_{o}=2.2 \mathrm{~mm}$. Les courants induits dans le creuset sont instantanément transformés en chaleur par effet Joule. Ensuite, cette chaleur est transmise par conduction dans tout le creuset. La chaleur transmise aux particules de coke se fait par 
conduction et rayonnement (pour une plus faible proportion). Les pertes de chaleur du creuset vers l'environnement sont minimisées par le placement d'un isolant entre les spires et le creuset.

\section{IV.3 Position des thermocouples}

Le gradient vertical a été déterminé en plaçant un thermocouple dans la partie supérieure de l'échantillon $\left(T_{d}\right)$ et un autre dans la partie inférieure $\left(\mathrm{T}_{\mathrm{f}}\right)$. Une attention particulière est accordée au gradient radial dans l'échantillon, étant donné que le gradient vertical est très faible, voire même négligeable, en milieu neutre comme en milieu oxydant. Le gradient vertical fluctue entre 2 et $4^{\circ} \mathrm{C}$.

Le gradient radial a été déterminé grâce à la pose de trois thermocouples: un thermocouple lit $T_{\text {ext }}$ sur la paroi interne du creuset du côté extérieur, un autre thermocouple lit $T_{c}$ la température au centre de l'échantillon et un troisième thermocouple lit $T_{\text {int }}$ la température sur la paroi interne du creuset du côté intérieur. La température $\mathrm{T}_{\mathrm{py}}$ est lue par le pyromètre sur la surface externe du creuset. La position des thermocouples est repérée sur la figure 4.1 . 


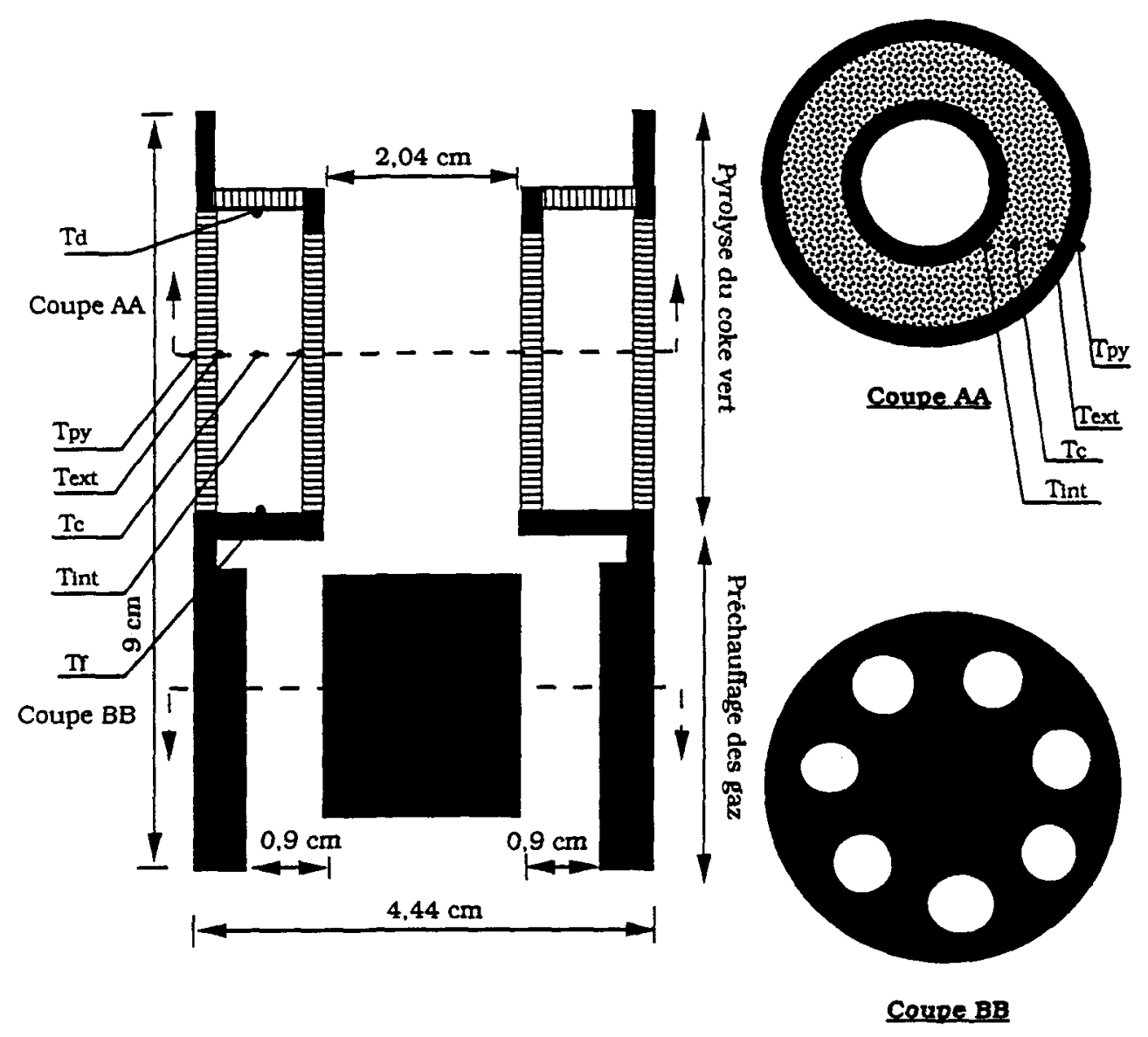

Figure 4.1: Position des thermocouples dans l'échantillon. 


\section{IV.4 Résultats}

\section{IV.4.1 Gradient radial en milieu neutre}

Les figures 4.2 à 4.4 donnent l'évolution des températures au cours du chauffage linéaire de l'échantillon en milieu neutre. Le tableau 4.1 récapitule les résultats concernant le gradient de température radial en milieu neutre. Dans les tableaux de résultats, a est la pente de la droite d'évolution de la température en ${ }^{\circ} \mathrm{C} / \mathrm{s}$ et $\mathrm{b}$, l'ordonnée à l'origine en ${ }^{\circ} \mathrm{C}$ (à $\mathrm{t}=0 \mathrm{~s}$ et $\mathrm{T}=450^{\circ} \mathrm{C}$ ).

\section{IV.4.2 Constatations en milieu neutre}

On peut constater que la température $T_{\text {int }}$ est très proche de la température au centre de l'échantillon $T_{c}$, ceci démontre l'efficacité de la section de préchauffage des gaz dans la partie inférieure du creuset. En effet, les gaz chauds arrivant au contact des particules contribuent à accroitre la température $T_{\text {int }}$ de sorte que durant tout le chauffage $T_{\text {int }}$ est même légèrement supérieur à $T_{c}$. Les deux droites $T_{i n t}$ et $T_{c}$ sont caractérisées par des pentes quasiment identiques (tableau 4.1); les deux températures suivent donc la même évolution au cours du chauffage.

D'autre part, on remarque que le faible gradient de température $T_{\text {int }}$ $T_{c}$ (environ $6^{\circ} \mathrm{C}$ ) ne varie pas pour les trois taux de chauffage.

Le gradient de température $T_{\text {ext }}-T_{c}$ diminue avec le taux de chauffage car un chauffage lent contribue à une meilleure répartition de la chaleur dans le lit de particules. Par contre, au cours du chauffage, le gradient $T_{\text {ext }}-T_{c}$ diminue progressivement et linéairement depuis $450^{\circ} \mathrm{C}$ où il est maximum jusqu'à $1200^{\circ} \mathrm{C}$ où il est minimum, et ce pour les trois taux de chauffage.

Dans les expériences de calcination, la température de l'échantillon est une température moyenne calculée en considérant un gradient radial moyen égal à $35^{\circ} \mathrm{C}$ (à $150^{\circ} \mathrm{C} / \mathrm{min}$ ), à $20^{\circ} \mathrm{C}\left(\right.$ à $100^{\circ} \mathrm{C} / \mathrm{min}$ ) et à $15^{\circ} \mathrm{C}$ (à $50^{\circ} \mathrm{C} / \mathrm{min}$ ). Ce gradient moyen est obtenu en se basant sur toutes les températures entre $T_{\text {ext }}$ et $T_{\text {int }}$. 


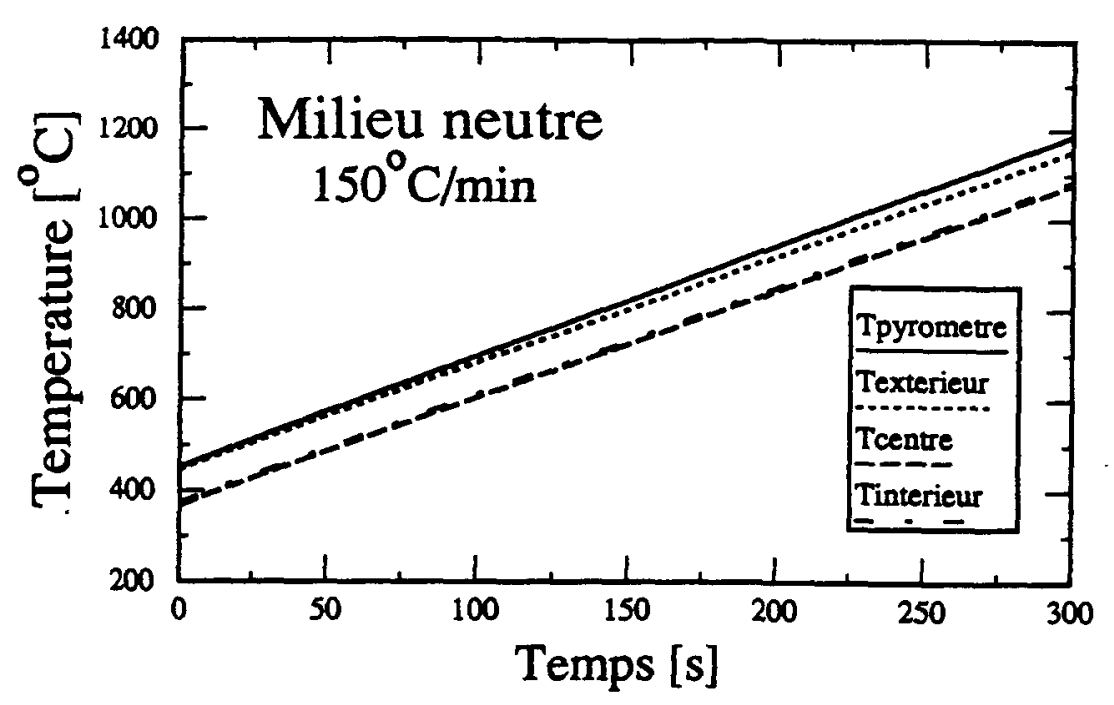

Figure 4.2 : Evolution des températures à $150^{\circ} \mathrm{C} / \mathrm{min}$, en milieu neutre (100\% azote).

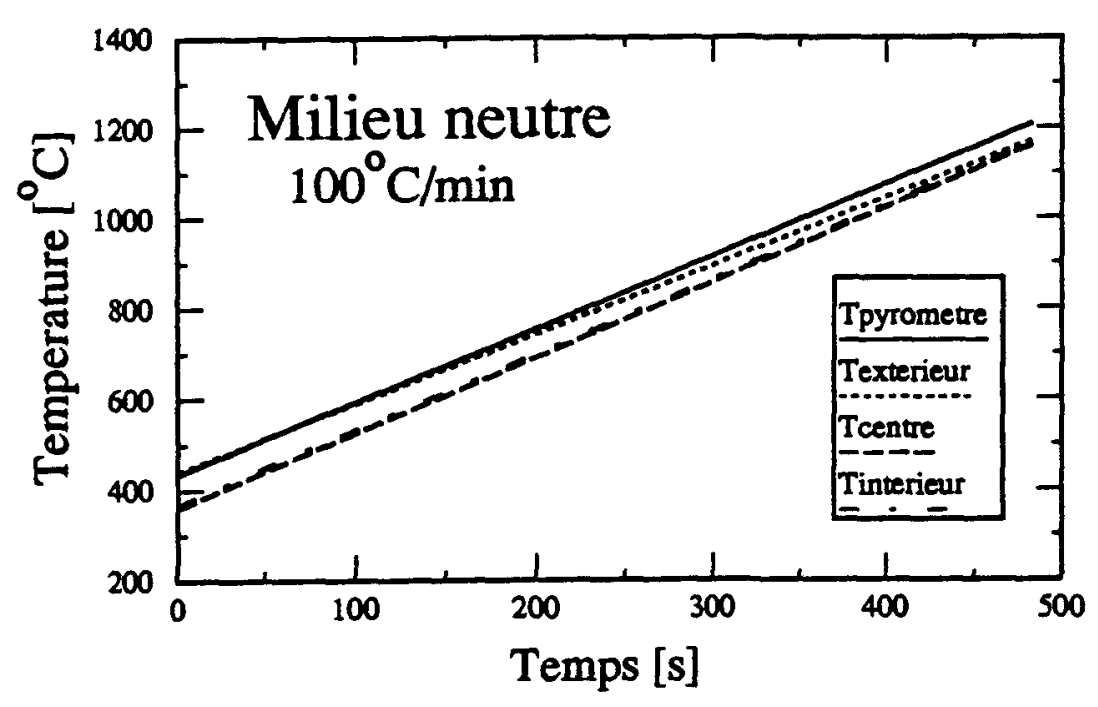

Figure 4.3: Evolution des températures à $100^{\circ} \mathrm{C} / \mathrm{min}$, en milieu neutre (100\% azote). 


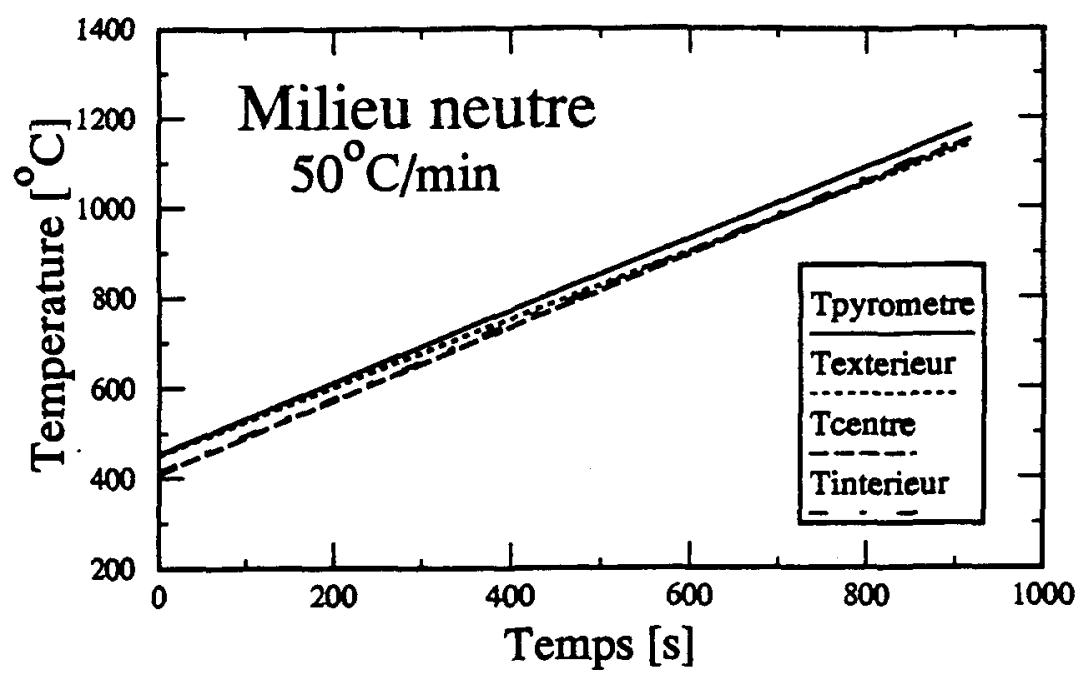

Figure 4.4: Evolution des températures à $50^{\circ} \mathrm{C} / \mathrm{min}$, en milieu neutre (100\% azote).

Tableau 4.1: $\quad$ Paramètres caractérisant l'évolution des températures en milieu neutre $\left(100 \% \mathrm{~N}_{2}\right)$.

\begin{tabular}{|c|c|c|c|c|}
\hline \multicolumn{5}{|c|}{$T=a t+b\left(^{*}\right)$} \\
\hline $\begin{array}{l}\text { Taux de } \\
\text { chauffage } \\
{\left[{ }^{\circ} \mathrm{C} / \mathrm{min}\right]}\end{array}$ & $\begin{array}{c}T_{\text {pyromètre }} \\
\text { a [ }\left[^{\circ} \mathrm{C} / \mathrm{s}\right] \\
\left.\mathrm{b} /{ }^{\circ} \mathrm{C}\right]\end{array}$ & Textérieur & $T_{\text {centre }}$ & $\mathrm{T}_{\text {intérieur }}$ \\
\hline 150 & $\begin{array}{l}a=2.45 \\
b=451.2\end{array}$ & $\begin{array}{l}a=2.35 \\
b=446.0\end{array}$ & $\begin{array}{l}a=2.37 \\
b=367.1\end{array}$ & $\begin{array}{l}a=2.38 \\
b=373.0\end{array}$ \\
\hline 100 & $\begin{array}{l}a=1.61 \\
b=433.0\end{array}$ & $\begin{array}{l}a=1.52 \\
b=438.7\end{array}$ & $\begin{array}{l}a=1.66 \\
b=358.6\end{array}$ & $\begin{array}{l}a=1.65 \\
b=366.9\end{array}$ \\
\hline 50 & $\begin{array}{l}a=0.79 \\
b=452.8\end{array}$ & $\begin{array}{l}a=0.71 \\
b=450.5\end{array}$ & $\begin{array}{l}a=0.81 \\
b=409.5\end{array}$ & $\begin{array}{l}a=0.81 \\
b=414.2\end{array}$ \\
\hline
\end{tabular}

$(*)$

$\begin{array}{lll}\mathrm{T} & \vdots & \text { Température }\left[{ }^{\circ} \mathrm{C}\right] \\ \mathrm{t} & \vdots & \text { Temps }[\mathrm{s}] \\ \mathrm{a} & \vdots & \text { Pente de la droite }\left[{ }^{\circ} \mathrm{C} / \mathrm{s}\right] \\ \mathrm{b} & \vdots & \text { Ordonnée à l'origine à } \mathrm{t}=0 \text { s et } \mathrm{T}=450^{\circ} \mathrm{C}\left[{ }^{\circ} \mathrm{C}\right]\end{array}$




\section{IV.4.3 Gradient radial en milieu oxydant}

Les figures 4.5 à 4.7 donnent l'évolution des températures au cours du chauffage linéaire en milieu oxydant. Le tableau 4.2 récapitule les résultats en milieu oxydant.

\section{IV.4.4 Constatations en milieu oxydant}

En milieu oxydant, la combustion des produits volatils émis par la dégazéification du coke vert et la combustion du carbone dégagent des quantités de chaleur importantes, contribuant à diminuer le gradient de température dans le lit de particules. Ces constatations ont été vérifiées expérimentalement.

Au taux de chauffage de $150^{\circ} \mathrm{C} / \mathrm{min}$ par exemple, le passage du milieu neutre au milieu oxydant diminue le gradient $\mathrm{T}_{\text {ext }}-\mathrm{T}_{\mathrm{c}}$ de $55 \%$. La contribution de la chaleur émise par les réactions exothermiques en présence d'oxygène n'est donc pas négligeable et améliore considérablement le gradient de température dans l'échantillon. Cette constatation n'a pas pu être mise en évidence à $100^{\circ} \mathrm{C} / \mathrm{min}$ mais il ne s'agit là que d'un fait isolé car il est difficile de reproduire exactement les mêmes conditions de travail en ce qui concerne la combustion du coke vert dans deux expériences successives à cause de la non homogénéité des échantillons de coke vert et d'une éventuelle faible variation du débit d'air.

Contrairement à ce que l'on avait pu observer en milieu neutre, en milieu oxydant la température au centre de l'échantillon $T_{c}$ est dans tous les cas supérieure à $T_{i n t}$, et ceci grâce au dégagement de chaleur de combustion. On a dans tous les cas $T_{c}>T_{i n t}$.

De plus, les deux droites $T_{c}$ et $T_{i n t}$ sont caractérisées par une pente supérieure pour $T_{c}$ par rapport à la pente de $T_{\text {int }}$; ce qui signifie que la température au centre de l'échantillon croît plus vite que $T_{\text {int }}$, de sorte que $\mathrm{T}_{\mathrm{c}}$ arrive à être supérieure à $\mathrm{T}_{\mathrm{py}}$ la température lue au pyromètre sur la surface extérieure du creuset (tableau 4.2). 
Il est important aussi de remarquer qu'en milieu oxydant, au cours du chauffage, la température $T_{\text {ext }}$ devient supérieure à la tempẻrature lue au pyromètre $T_{p y}$. La pente de la droite $T_{\text {ext }}$ est supérieure à la pente de la droite $\mathrm{T}_{\mathrm{py}}$; ce qui signifie que le taux de chauffage des particules situées dans l'environnement immédiat de la paroi externe du creuset est supérieur au taux de chauffage global imposé par le contrôleur de température relié au pyromètre et au générateur de hautes fréquences.

Dans les expériences de calcination, la température de l'échantillon est une température moyenne calculée en considérant un gradient radial moyen égal à $\left.3^{\circ} \mathrm{C}(a ̀) 150^{\circ} \mathrm{C} / \mathrm{min}\right)$, à $18^{\circ} \mathrm{C}\left(\mathbf{a} 100^{\circ} \mathrm{C} / \mathrm{min}\right)$ et à $8^{\circ} \mathrm{C}$ (à $\left.50^{\circ} \mathrm{C} / \mathrm{min}\right)$.

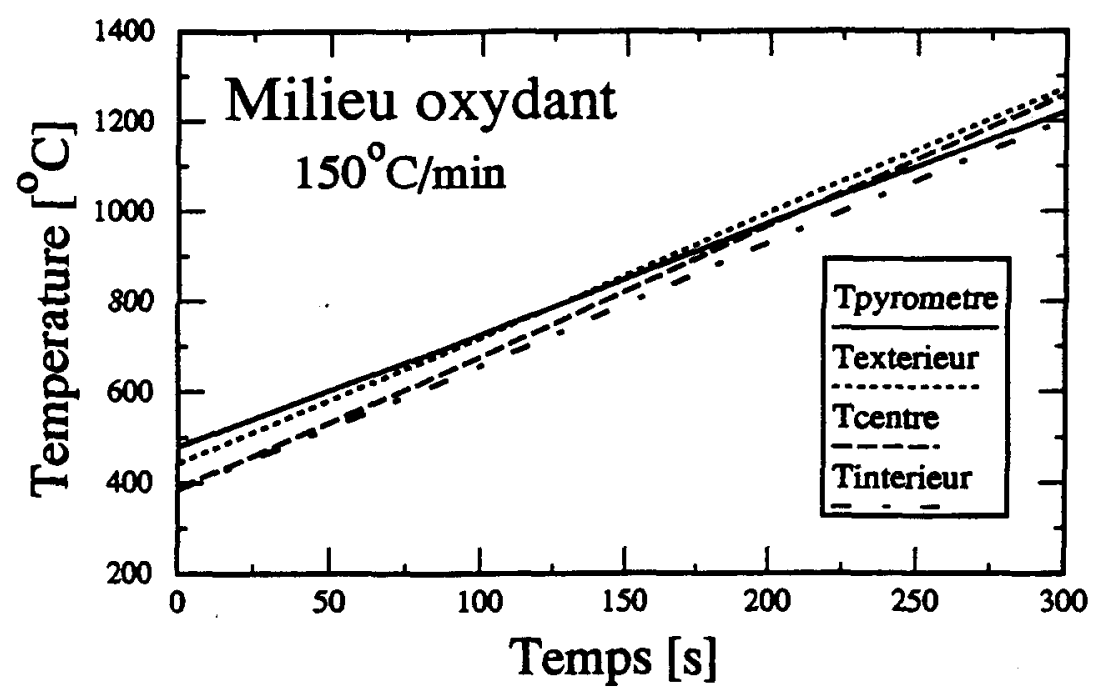

Figure 4.5: Evolution des températures à $150^{\circ} \mathrm{C} / \mathrm{min}$, en milieu oxydant (95\% azote, $5 \%$ oxygène). 


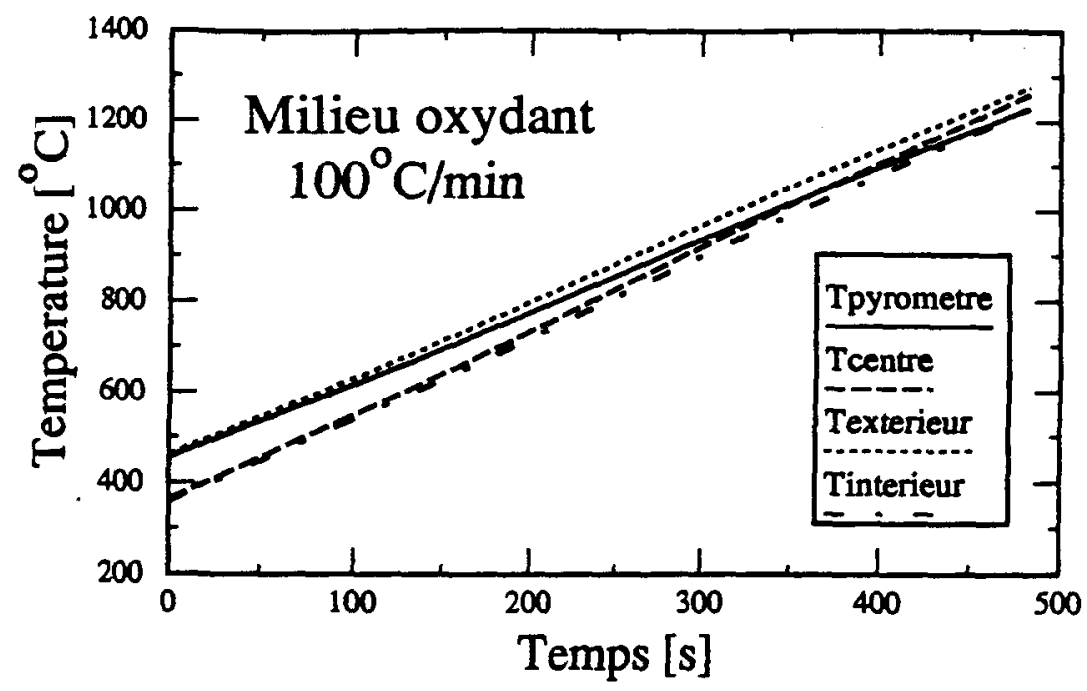

Figure 4.6 : Evolution des températures à $100^{\circ} \mathrm{C} / \mathrm{min}$, en milieu oxydant ( $95 \%$ azote, $5 \%$ oxygène).

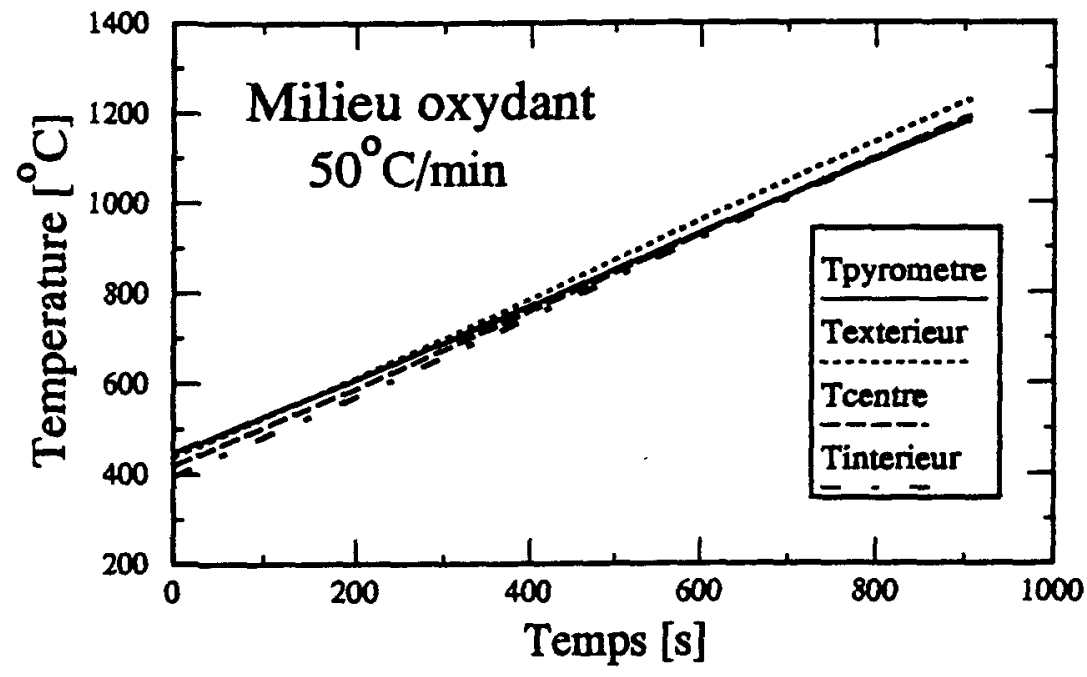

Figure 4.7: Evolution des températures à $50^{\circ} \mathrm{C} / \mathrm{min}$, en milieu oxydant ( $95 \%$ azote, $5 \%$ oxygène). 
Tableau 4.2: $\quad$ Paramètres caractérisant l'évolution des températures en milieu oxydant $\left(5 \% \mathrm{O}_{2}-95 \% \mathrm{~N}_{2}\right)$.

\begin{tabular}{|c|c|c|c|c|}
\hline \multicolumn{5}{|c|}{$\mathbf{T}=\mathbf{a} \mathbf{t}+\mathbf{b}\left(^{*}\right)$} \\
\hline $\begin{array}{l}\text { Taux de } \\
\text { chauffage } \\
{\left[{ }^{\circ} \mathrm{C} / \mathrm{min}\right]}\end{array}$ & $\begin{array}{c}\text { Tpyromètre } \\
\left.\text { a [ }{ }^{\circ} \mathrm{C} / \mathrm{s}\right] \\
\mathrm{b}\left[{ }^{\circ} \mathrm{C}\right]\end{array}$ & $T_{\text {extérieur }}$ & $\mathrm{T}_{\text {centre }}$ & $T_{\text {intérieur }}$ \\
\hline 150 & $\begin{array}{l}a=2.47 \\
b=479.1\end{array}$ & $\begin{array}{l}\mathrm{a}=2.77 \\
\mathrm{~b}=442.4\end{array}$ & $\begin{array}{l}a=2.91 \\
b=388\end{array}$ & $\begin{array}{l}a=2.73 \\
b=384\end{array}$ \\
\hline 100 & $\begin{array}{l}a=1.61 \\
b=454.9\end{array}$ & $\begin{array}{l}a=1.68 \\
b=460.7\end{array}$ & $\begin{array}{l}a=1.86 \\
b=362.3\end{array}$ & $\begin{array}{l}a=1.81 \\
b=357.9\end{array}$ \\
\hline 50 & $\begin{array}{l}a=0.81 \\
b=446.3\end{array}$ & $\begin{array}{l}a=0.87 \\
b=437.8\end{array}$ & $\begin{array}{l}a=0.85 \\
b=418.2\end{array}$ & $\begin{array}{l}a=0.88 \\
b=394.8\end{array}$ \\
\hline$(*)$ & $\begin{array}{l}\mathrm{T} \\
\mathrm{t} \\
\mathrm{a} \\
\mathrm{b}\end{array}$ & $\begin{array}{l}\text { Température } \\
\text { Temps [s] } \\
\text { Pente de la dr } \\
\text { Ordonnée à l'c }\end{array}$ & $\begin{array}{l}\mathrm{C} / \mathrm{sl} \\
\mathrm{e} \text { a } t=0 \text { s et } 7\end{array}$ & ${ }^{\circ} \mathrm{C}\left[{ }^{\circ} \mathrm{C}\right]$ \\
\hline
\end{tabular}

\section{IV.5 Conclusions}

D'une manière générale, on peut dire que le passage du milieu neutre au milieu oxydant réduit considérablement le gradient radial de température dans l'échantillon grâce aux réactions exothermiques de combustion. Le gradient vertical par contre est négligeable dans les deux milieux. On constate que le gradient radial diminue avec le taux de chauffage et on peut affirmer qu'il est acceptable dans les conditions de travail que sont les nôtres. En effet, l'hétérogénéité du milieu de travail nous impose la conception d'un creuset "ouvert" permettant un contact entre le solide et les gaz. On se trouve donc face à un compromis entre les limites d'un gradient de température acceptable et la forme à donner au creuset pour respecter les exigences de la cinétique hétérogène.

Tous les résultats présentés dans cet ouvrage ont été corrigés pour le gradient de température radial dans l'échantillon. 
CHAPITRE V

\section{PYROLYSE DU COKE VERT EN MILIEU NEUTRE}

\section{V.1 Introduction}

Si le thermogramme obtenu par la pyrolyse dépend de la nature du corps qui en est l'objet, il dépend beaucoup également des conditions dans lesquelles cette pyrolyse est effectuée. Aussi, est-il vain de vouloir caractériser la pyrolyse d'un corps par un thermogramme unique, établi une fois pour toute car ce thermogramme n'aurait de valeur que dans les conditions très précises où il aurait été obtenu. Parmi les éléments prépondérants influençant l'allure du thermogramme, citons par exemple la forme du creuset, la vitesse de chauffage et la nature de l'atmosphère dans laquelle l'expérience est menée.

Une première étude préliminaire est réalisée sur le coke TEXACO pour déterminer l'influence éventuelle de la taille des particules sur les courbes thermogravimétriques et les gaz recueillis, en comparant le coke de granulométrie fine $(-20+35$ mailles Tyler: $0.42-0.83 \mathrm{~mm})$ dans un creuset de type "fermé" [2,3], avec le coke de granulométrie plus grossière $(-8+10$ mailles Tyler: 1.65-2.36mm) dans le même creuset (paragraphe V.2).

Une seconde étude préliminaire est menée afin d'apprécier l'effet de la forme du creuset sur les résultats en comparant le creuset de type "fermé" [2,3] avec le creuset de type "ouvert" mis au point dans le cadre de ce travail (paragraphe V.3).

Les thermogrammes et l'analyse des gaz en milieu neutre sont présentés dans les paragraphes V.4 et V.5 respectivement. L'influence du taux de chauffage sur l'émission des gaz est détaillée pour le coke TEXACO au paragraphe V.2.2; les trois autres cokes sont traités en annexe I. En ce qui concerne le modèle cinétique de dévolatilisation, les résultats sont présentés au paragraphe V.6 pour le coke TEXACO à $150^{\circ} \mathrm{C} / \mathrm{min}$; les 
résultats obtenus aux deux autres taux de chauffage se trouvent en annexes II et III.

\section{V.2 Influence de la taille des particules sur la cinétique de dévolatilisation}

Les expériences préliminaires sont menées dans les mêmes conditions que celles de $\mathrm{D}$. Kocaefe et $\mathrm{A}$. Charette dans une étude antérieure en milieu neutre [2,3], avec du coke de granulométrie grossière, dans un creuset de type "fermé".

Les conditions expérimentales sont les suivantes:

Creuset

Coke vert TEXACO

Poids échantillon

Débit d'azote

Débit d'aspiration des gaz :

Taux de chauffage
: $\quad$ type "fermé" $[2,3]$

: $\quad-8+10$ mailles $(1.65-2.36 \mathrm{~mm})$

: $20 \mathrm{~g}$

: $\quad 10.6 \mathrm{~L} / \mathrm{min}\left(\right.$ à $0^{\circ} \mathrm{C}$ et $\left.1 \mathrm{~atm}\right)$

$313 \mathrm{~cm}^{3} / \mathrm{min}$

$150^{\circ} \mathrm{C} / \mathrm{min}, 100^{\circ} \mathrm{C} / \mathrm{min}$ et $50^{\circ} \mathrm{C} / \mathrm{min}$

On constate que le changement de granulométrie $-20+35$ mailles pour $-8+10$ mailles n'a aucune influence en ce qui concerne les courbes thermogravimétriques et les gaz recueillis.

\section{V.3 Influence de la forme du creuset sur la cinétique de dévolatilisation}

- On démontre expérimentalement que la forme du creuset influence considérablement les courbes thermogravimétriques ainsi que les gaz recueillis. En effet, le creuset de type "ouvert" permet une évacuation plus aisée des produits volatils formés au cours de la calcination du coke, comparativement au creuset de type "fermé" [2,3]. Ainsi, la concentration des gaz est plus importante car les gaz de dévolatilisation du coke sont directement evacués à l'extérieur du creuset par les nombreux orifices percés dans celui-ci. Il faut aussi remarquer que les particules $-8+10$ 
mailles étant plus grosses, les porosités interstitielles sont donc de diamètre plus important, comparativement au $-20+35$ mailles, ce qui améliore la diffusion des volatils au travers du lit de particules.

Les conditions expérimentales en milieu neutre sont les suivantes:

$\begin{array}{lcl}\text { Creuset } & : & \text { type"ouvert" } \\ \text { Coke TEXACO } & : & -8+10 \text { mailles }(1.65-2.36 \mathrm{~mm}) \\ \text { Poids échantillon } & : & 10 \mathrm{~g} \\ \text { Débit d'azote } & : & 10.6 \mathrm{~L} / \mathrm{min}\left(\mathrm{à} 0^{\circ} \mathrm{C} \text { et } 1 \mathrm{~atm}\right) \\ \text { Débit d'aspiration des gaz } & : & 313 \mathrm{~cm} / \mathrm{min} \\ \text { Taux de chauffage } & : & 150^{\circ} \mathrm{C} / \mathrm{min}, 100^{\circ} \mathrm{C} / \mathrm{min} \text { et } 50^{\circ} \mathrm{C} / \mathrm{min}\end{array}$

Ces conditions expérimentales ont été établies en se basant sur des expériences préliminaires visant à déterminer l'influence de la taille des particules et de la forme du creuset.

\section{V.4 Résultats thermogravimétriques}

\section{V.4.1 Comparaison des cokes}

Les figures $5.1,5.2$ et 5.3 comparent les thermogrammes obtenus en milieu neutre sur les quatre types de coke, et ce pour les trois taux de chauffage respectivement. La perte de poids la plus élevée est observée pour le coke YPF; la plus faible est observée pour le coke CONOCO; les cokes TEXACO et RTW étant caractérisés par des thermogrammes très proches. L'allure des thermogrammes est reliée aux propriẻtés intrinsèques des cokes verts; la perte de poids la plus élevée est observée pour le coke contenant le plus haut pourcentage de matières volatiles. Le tableau 5.1 donne le pourcentage de perte de poids total observé à $1200^{\circ} \mathrm{C}$, pour les quatre types de coke et les trois taux de chauffage. 


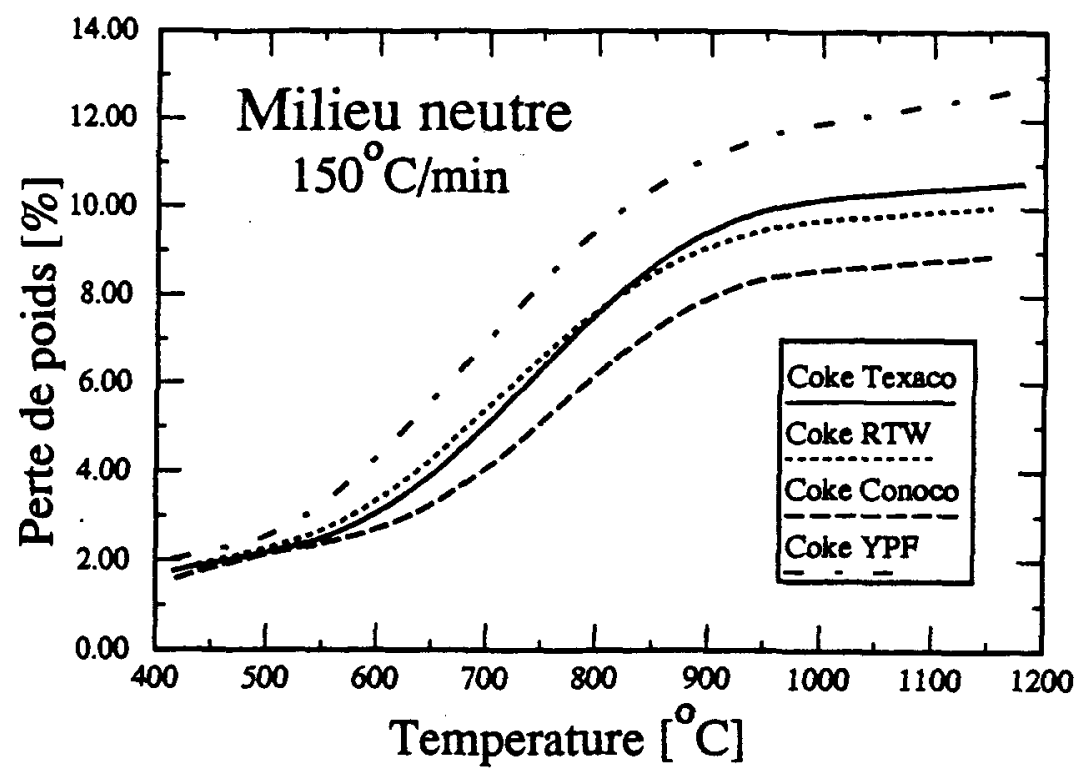

Figure 5.1: Comparaison des thermogrammes à $150^{\circ} \mathrm{C} / \mathrm{min}$, en milieu neutre (100\% azote).

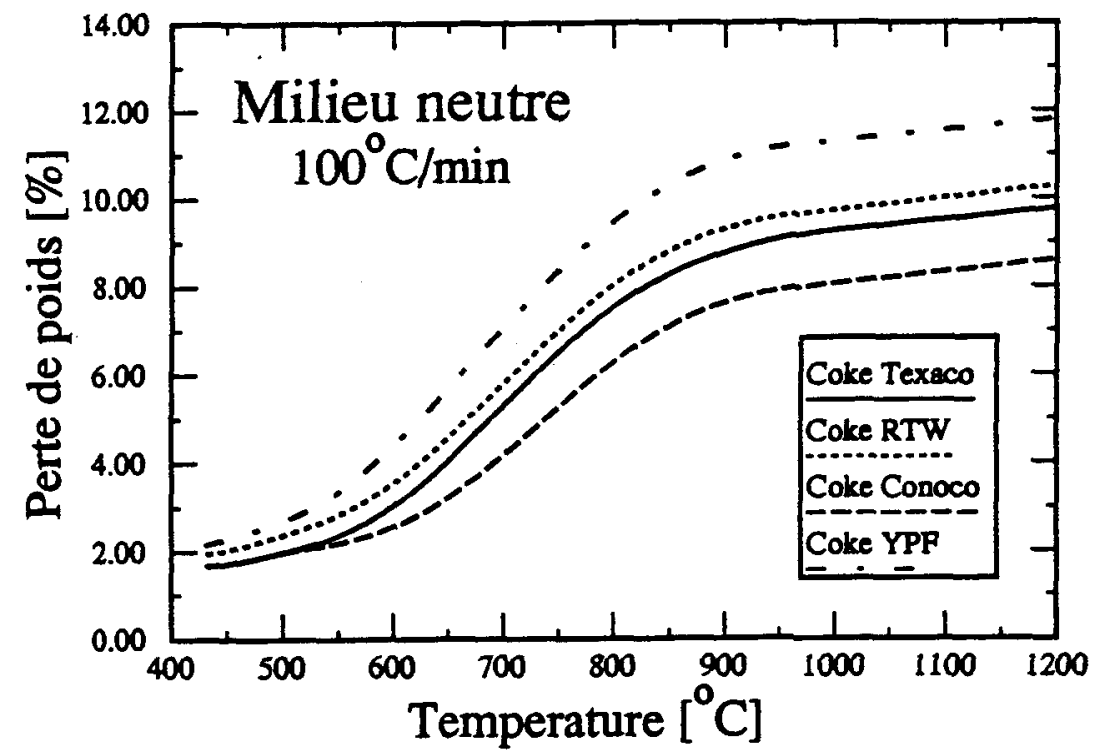

Figure 5.2: Comparaison des thermogarmmes à $100^{\circ} \mathrm{C} / \mathrm{min}$, en milieu neutre $(100 \%$ azote). 


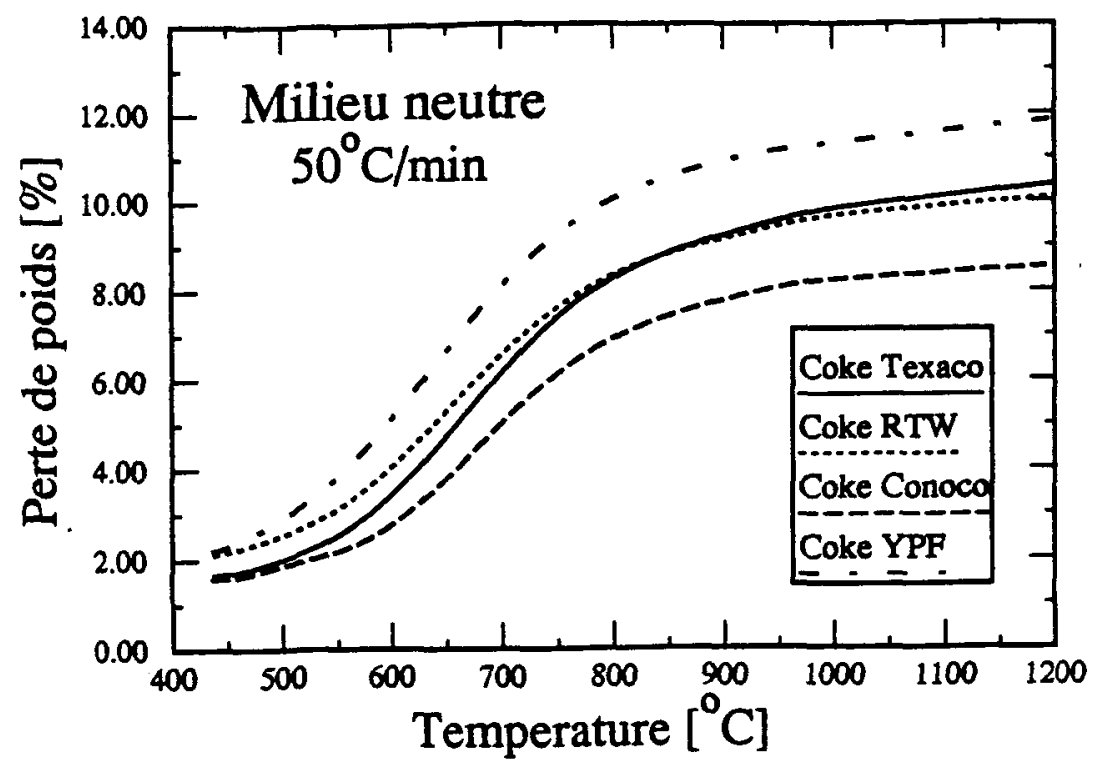

Figure 5.3: Comparaison des thermogrammes à $50^{\circ} \mathrm{C} / \mathrm{min}$, en milieu neutre $(100 \%$ azote).

Tableau 5.1: $\quad$ Perte de poids totale en fin de calcination $\left(1200^{\circ} \mathrm{C}\right)$, en milieu neutre $\left(100 \% \mathrm{~N}_{2}\right)$.

\begin{tabular}{|l|c|c|}
\hline Cokes & $\begin{array}{c}\text { Taux de chauffage } \\
{\left[{ }^{\circ} \mathrm{C} / \mathrm{min}\right]}\end{array}$ & $\begin{array}{c}\text { Perte de poids } \\
{[\%]}\end{array}$ \\
\hline TEXACO & 153.2 & 10.57 \\
& 98.5 & 9.77 \\
& 48.7 & 10.40 \\
\hline RTW & 147.5 & 10.20 \\
& 100.8 & 10.30 \\
& 49.7 & 10.11 \\
\hline YPF & 150.6 & 12.65 \\
& 97.2 & 11.82 \\
& 48.0 & 11.83 \\
\hline CONOCO & 147.2 & 8.89 \\
& 97.4 & 8.62 \\
& 47.8 & 8.52 \\
\hline
\end{tabular}




\section{V.4.2 Effet du taux de chauffage}

Les figures 5.4 à 5.7 présentent les thermogrammes obtenus pour les trois taux de chauffage et ce respectivement pour les quatre types de coke. Un taux de chauffage élevé retarde la dévolatilisation du coke vert au cours de sa calcination [22].

Comme il a déjà été constaté auparavant par plusieurs auteurs, on voit qu'à basse tempêrature $\left(<900^{\circ} \mathrm{C}\right)$, le pourcentage de perte de poids augmente avec la diminution du taux de chauffage [2,22]. Plus la durée du traitement thermique est courte (haut taux de chauffage), plus élevée est la température requise pour atteindre un niveau de dévolatilisation fixé [22].

Par contre, à haute température $\left(>900^{\circ} \mathrm{C}\right)$, le pourcentage de perte de poids augmente avec le taux de chauffage. La perte de poids totale est plus élevée pour le taux de chauffage le plus élevé $\left(150^{\circ} \mathrm{C} / \mathrm{min}\right)$. Ceci a d'ailleurs déjà été observê dans des études antérieures [22].

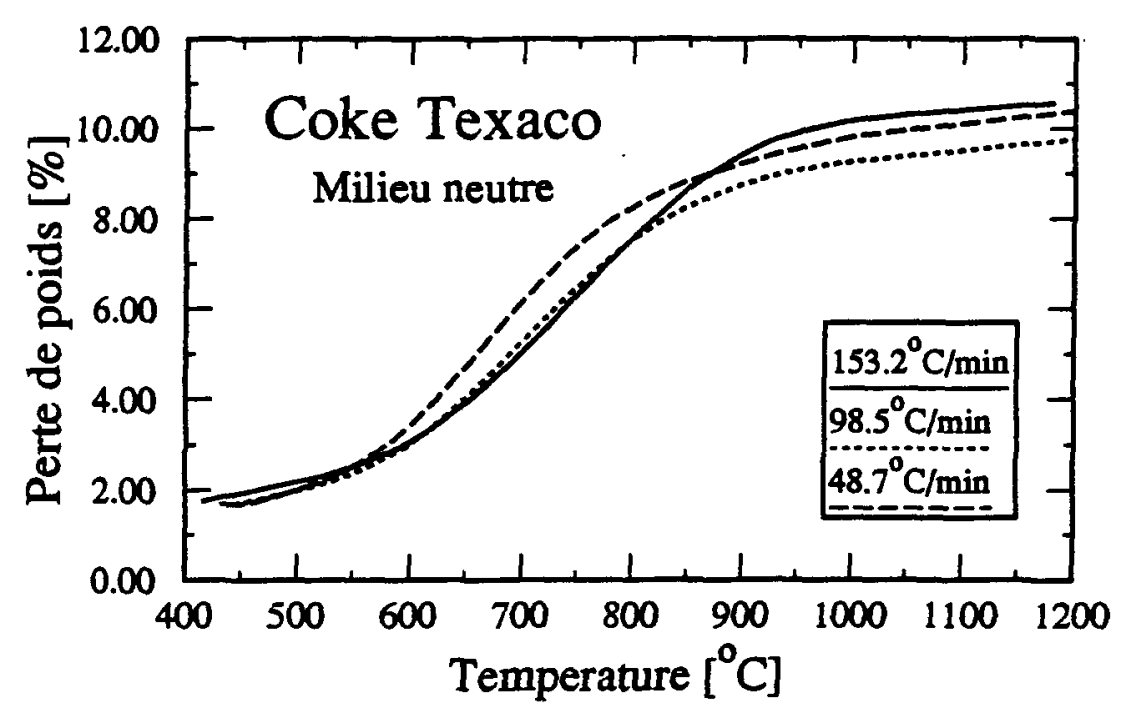

Figure 5.4: Effet du taux de chauffage sur le thermogramme du coke TEXACO, en milieu neutre ( $100 \%$ azote). 


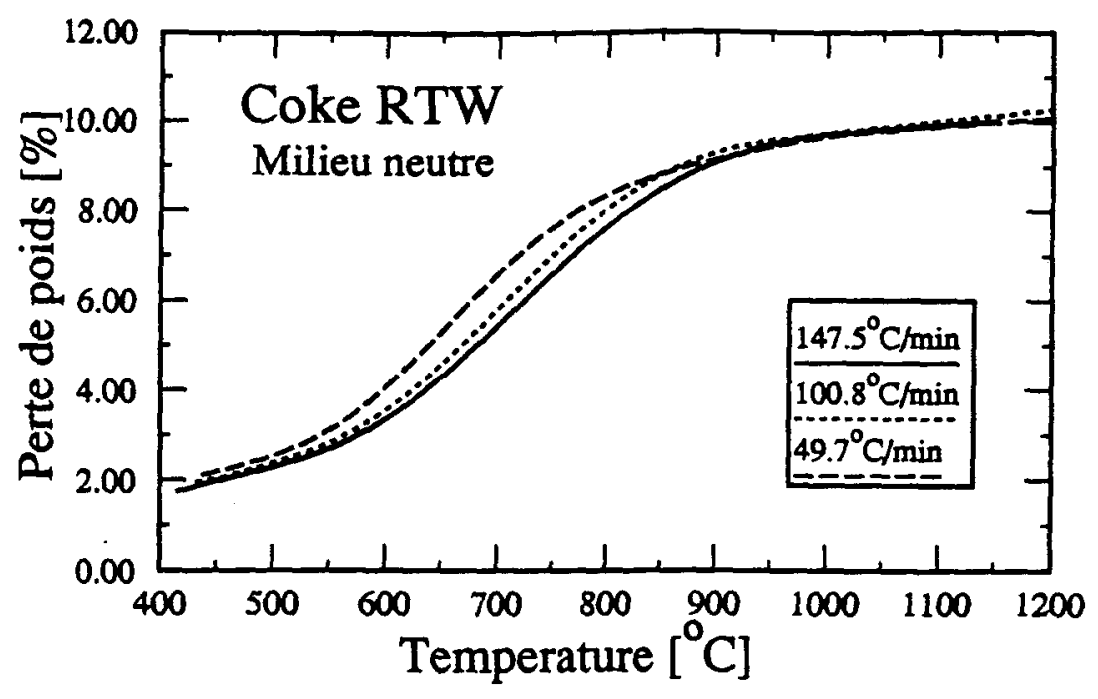

Figure 5.5: Effet du taux de chauffage sur le thermogramme du coke RTW, en milieu neutre (100\% azote).

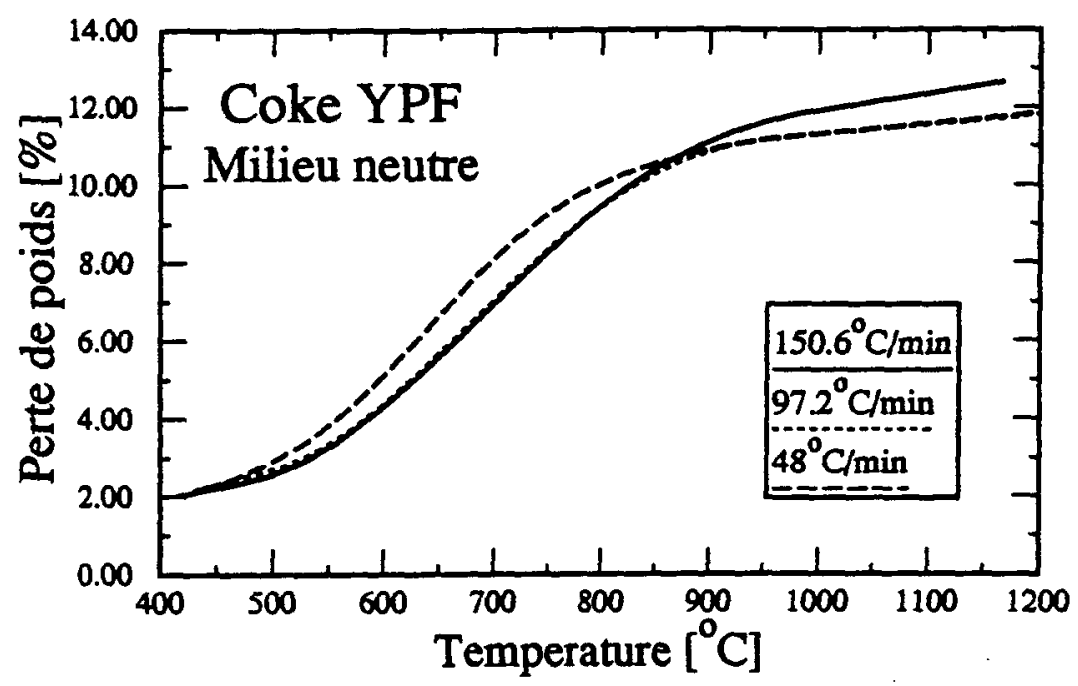

Figure 5.6 : Effet du taux de chauffage sur le thermogramme du coke YPF, en milieu neutre ( $100 \%$ azote). 


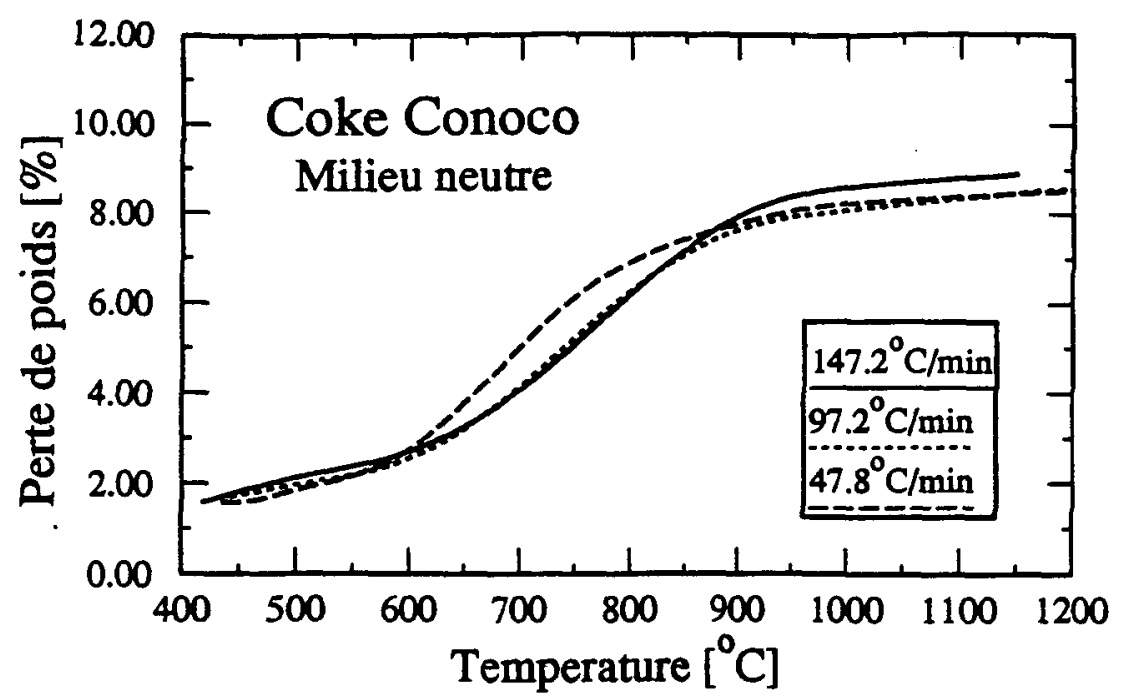

Figure 5.7: Effet du taux de chauffage sur le thermogramme du coke CONOCO, en milieu neutre ( $100 \%$ azote).

\section{V.5 Analyse des gaz}

\section{V.5.1 Comparaison des cokes}

Les figures $5.8,5.9$ et 5.10 donnent les concentrations d'hydrogène et de méthane obtenues pour les quatres types de coke et ce pour les trois taux de chauffage respectivement. La concentration des gaz est exprimée en ppm (part par million) et rapportée à $100 \mathrm{~g}$ de coke vert.

L'aire sous la courbe est proportionnelle à la perte de poids cumulative due au départ des produits volatils $\mathrm{CH}_{4}$ et $\mathrm{H}_{2}$. L'aire la plus élevée est observée pour le coke YPF contenant le plus haut pourcentage de matières volatiles; la plus petite aire est observée pour le coke CONOCO. La concentration maximale en $\mathrm{CH}_{4}$ est observée à la même température quel que soit le type de coke vert; il en est de même pour la concentration maximale en $\mathrm{H}_{2}$. On constate que la dévolatilisation n'est pas complète en ce qui concerne l'hydrogène, une fois la température de $1200^{\circ} \mathrm{C}$ atteinte. Par contre, pour le $\mathrm{CH}_{4}$, la dévolatilisation est complète aux alentours de $1000^{\circ} \mathrm{C}$. 


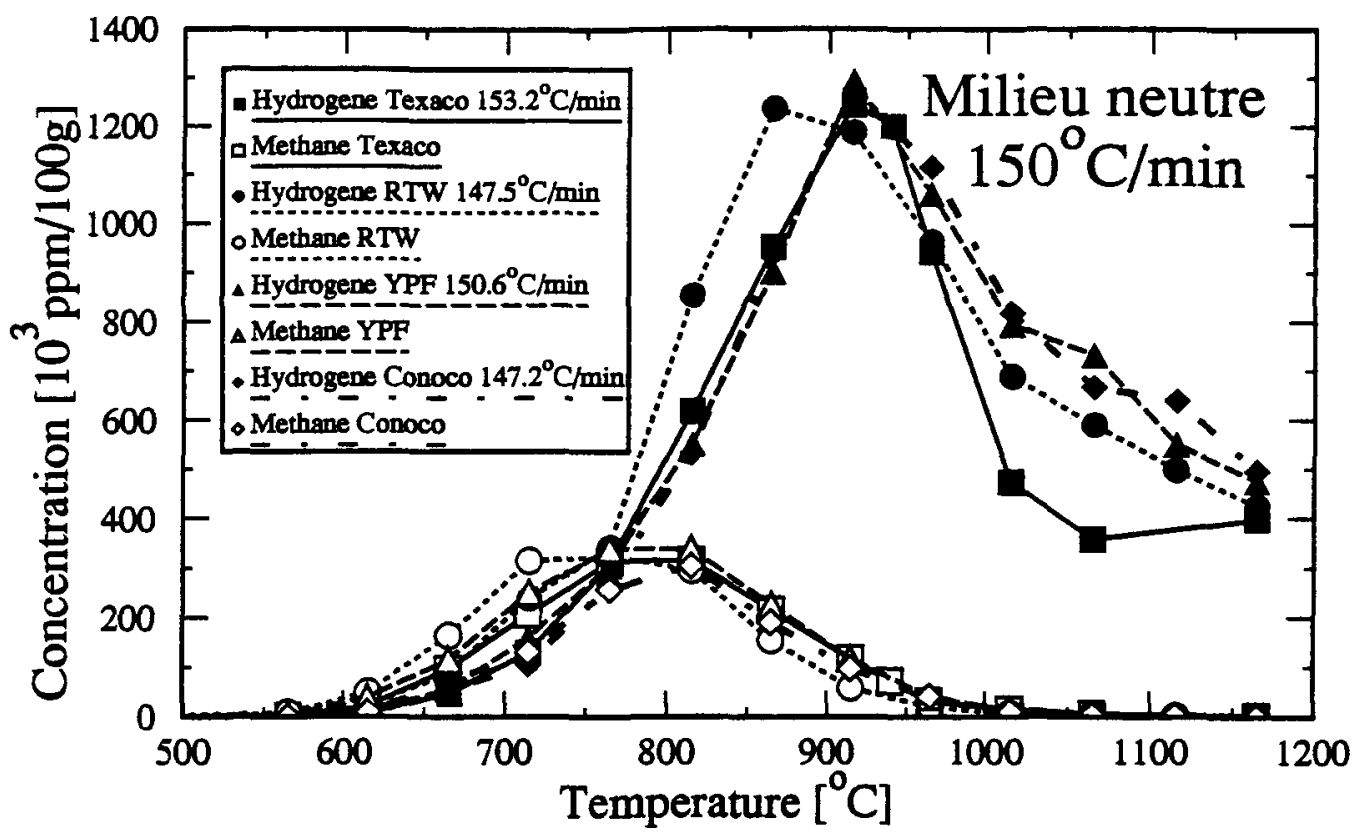

Figure 5.8: Comparaison de l'hydrogène et du méthane à $150^{\circ} \mathrm{C} / \mathrm{min}$, en milleu neutre (100\% azote).

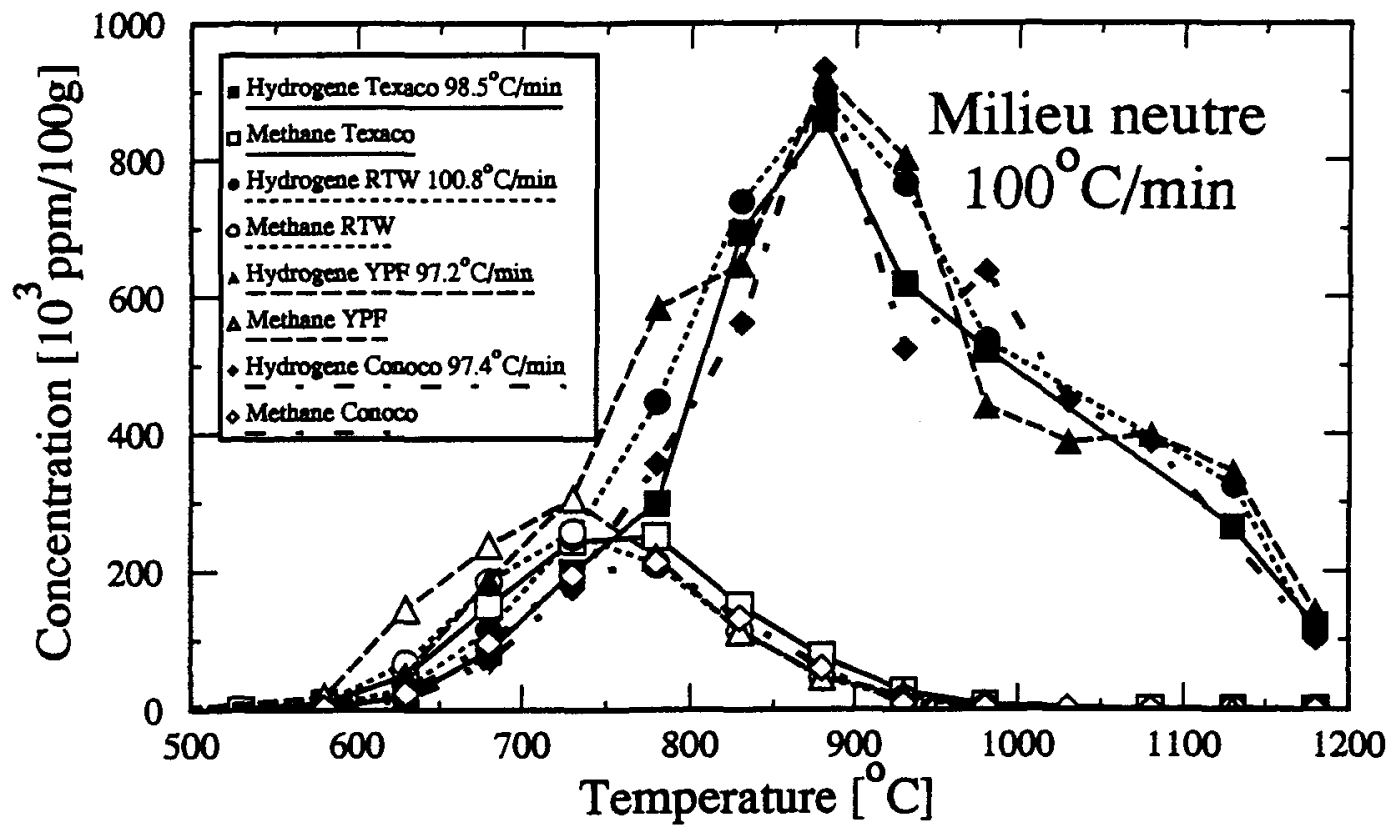

Figure 5.9 : Comparaison de $1^{\prime h y d r o g e ̀ n e ~ e t ~ d u ~ m e ́ t h a n e ~ a ̀ ~} 100^{\circ} \mathrm{C} / \mathrm{min}$, en milieu neutre (100\% azote). 




Figure 5.10: Comparaison de l'hydrogène et du méthane à $50^{\circ} \mathrm{C} / \mathrm{min}$, en milieu neutre (100\% azote).

\section{V.5.2 Effet du taux de chauffage}

Les figures 5.11 et 5.12 révèlent l'effet du taux de chauffage sur les concentrations en $\mathrm{H}_{2}$ et en $\mathrm{CH}_{4}$ pour le coke TEXACO.

On constate que le dégagement de méthane et d'hydrogène débute plus tôt lorsque le taux de chauffage est plus faible. Un taux de chauffage élevé engendre un retard dans l'émission des produits volatils $[2,22]$. Pour $\mathrm{l}^{\prime} \mathrm{H}_{2}$, le maximum est observé à $900^{\circ} \mathrm{C}$ à $150^{\circ} \mathrm{C} / \mathrm{min}$, à $850^{\circ} \mathrm{C}$ à $100^{\circ} \mathrm{C} / \mathrm{min}$ et à $800^{\circ} \mathrm{C}$ à $50^{\circ} \mathrm{C} / \mathrm{min}$. Pour le $\mathrm{CH}_{4}$, le maximum est observé à $800^{\circ} \mathrm{C}$ à $150^{\circ} \mathrm{C} / \mathrm{min}$, à $750^{\circ} \mathrm{C}$ à $100^{\circ} \mathrm{C} / \mathrm{min}$ et à $700^{\circ} \mathrm{C}$ à $50^{\circ} \mathrm{C} / \mathrm{min}$. Le maximum se déplace vers les hautes températures, lorsque le taux de chauffage croît.

Il semblerait, au regard des figures 5.11 et 5.12, que la quantité de gaz émis augmente avec le taux de chauffage (aire sous la courbe plus grande pour $150^{\circ} \mathrm{C} / \mathrm{min}$ ); or ce n'est pas le cas du tout car il faut comparer l'aire sous la courbe en fonction du temps et non de la température. En effet, les 
valeurs instantanées sont plus élevées à $150^{\circ} \mathrm{C} / \mathrm{min}$; toutefois, la durée de $l^{\prime}$ expérience est de 300 secondes approximativement. Pour $100^{\circ} \mathrm{C} / \mathrm{min}$ et $50^{\circ} \mathrm{C} / \mathrm{min}$, la durée de l'expérience est de 450 et 950 secondes respectivement. Les figures 5.13 et 5.14 présentent les concentrations des gaz $\mathrm{H}_{2}$ et $\mathrm{CH}_{4}$ en fonction du temps pour les trois taux de chauffage. On constate bien que les valeurs instantanées diminuent avec le taux de chauffage mais les valeurs cumulatives (aire sous la courbe) augmentent avec la diminution du taux de chauffage. La même tendance en ce qui concerne l'effet du taux de chauffage sur la concentration des gaz recueillis est observée pour les trois autres types de coke (annexe I).

La figure 5.15 montre l'effet du taux de chauffage sur le CO recueilli au cours de la pyrolyse du coke TEXACO, en milieu neutre. Comme déjà observé pour les autres gaz (hydrogène et méthane), on remarque que le pic du CO se déplace vers les hautes températures et que son amplitude croit avec la vitesse de chauffage. Le monoxyde de carbone dégagé lors du traitement thermique du coke en milieu neutre provient exclusivement de la réaction entre le carbone et l'oxygène atomique qui lui est intimement lié.

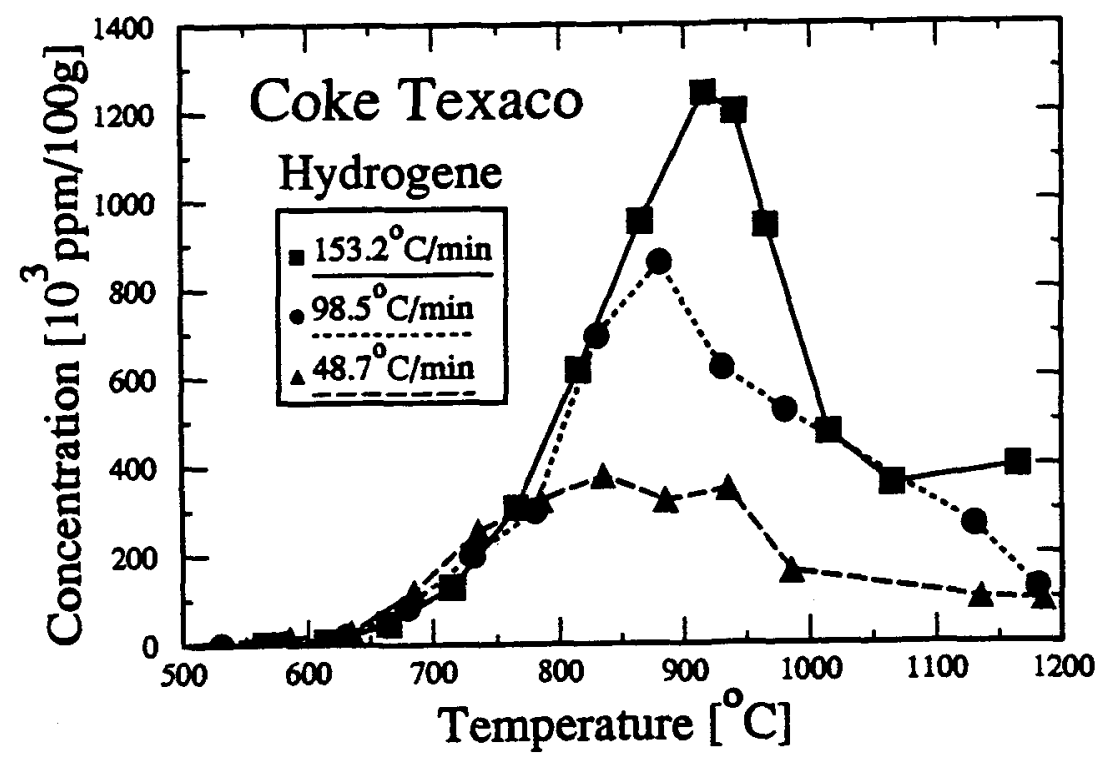

Figure 5.11 : Effet du taux de chauffage sur l'hydrogène du coke TEXACO, en milieu neutre (100\% azote). 


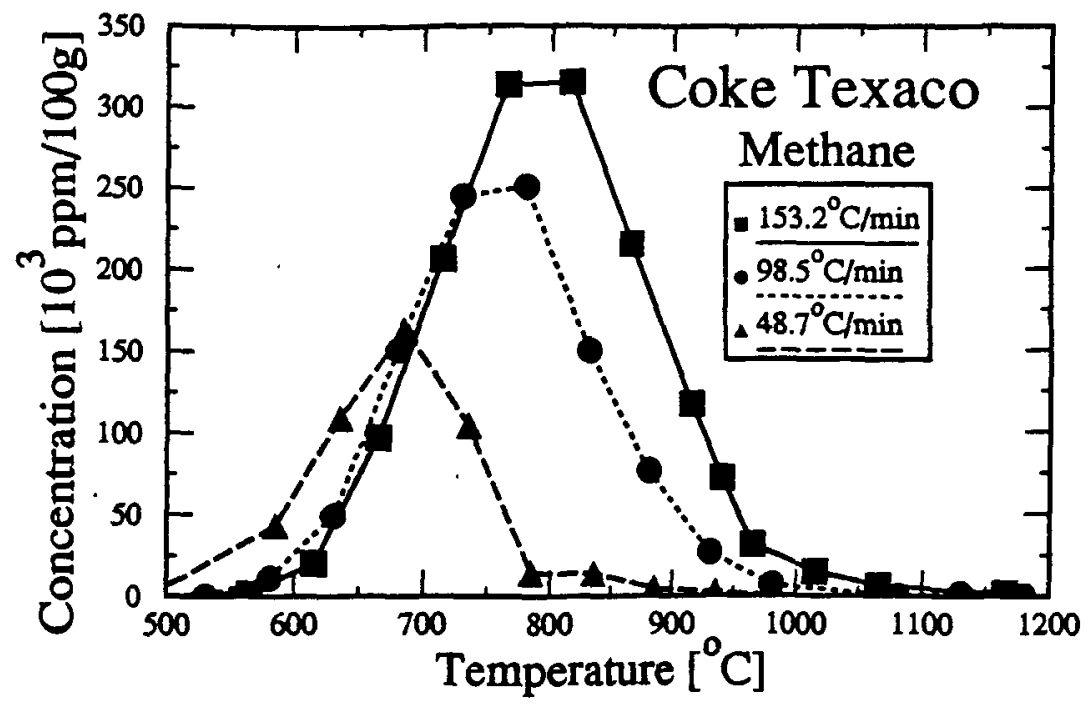

Figure 5.12: Effet du taux de chauffage sur le méthane du coke TEXACO, en milieu neutre (100\% azote).

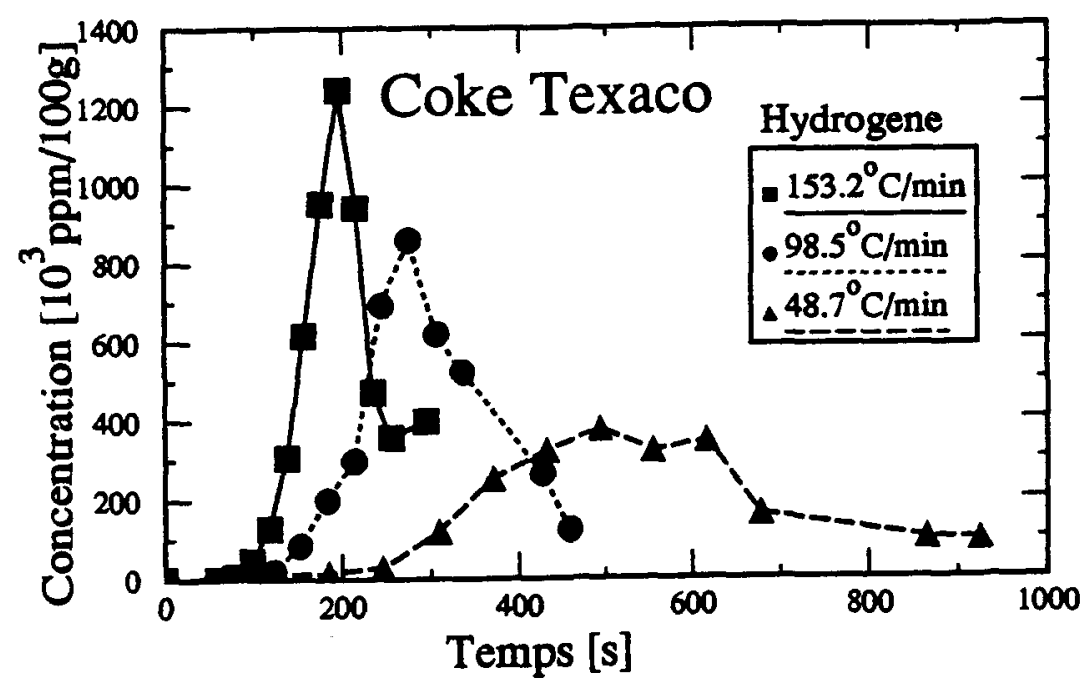

Figure 5.13: Effet du taux de chauffage sur l'hydrogène du coke TEXACO, en fonction du temps. 


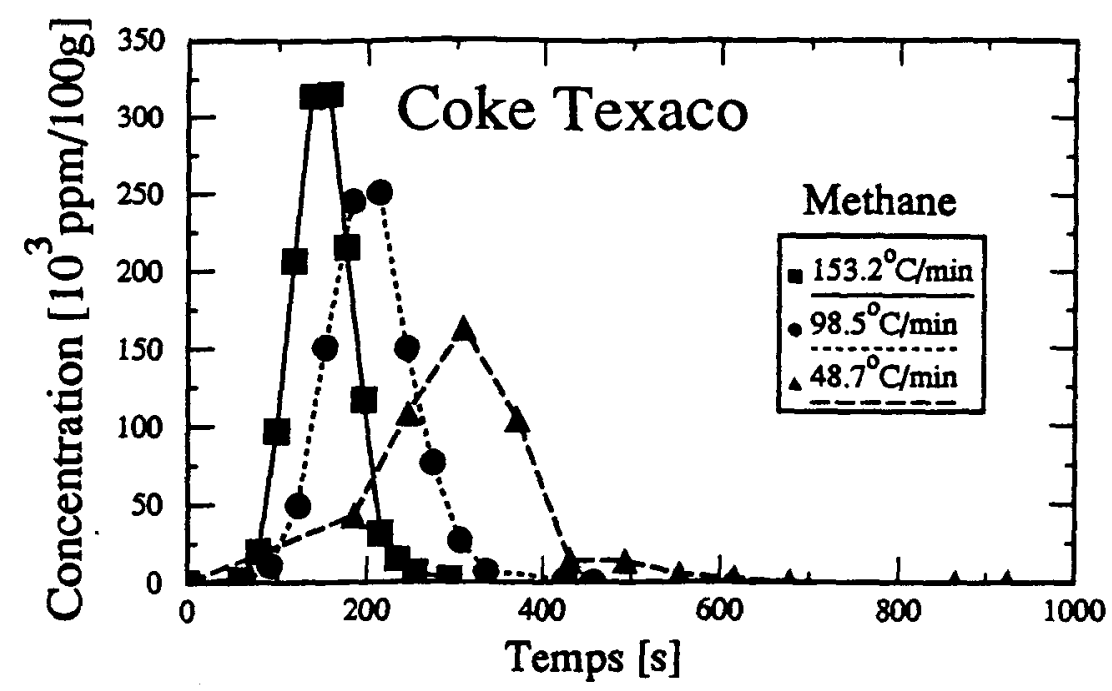

Figure 5.14: Effet du taux de chauffage sur le mëthane du coke TEXACO, en fonction du temps.

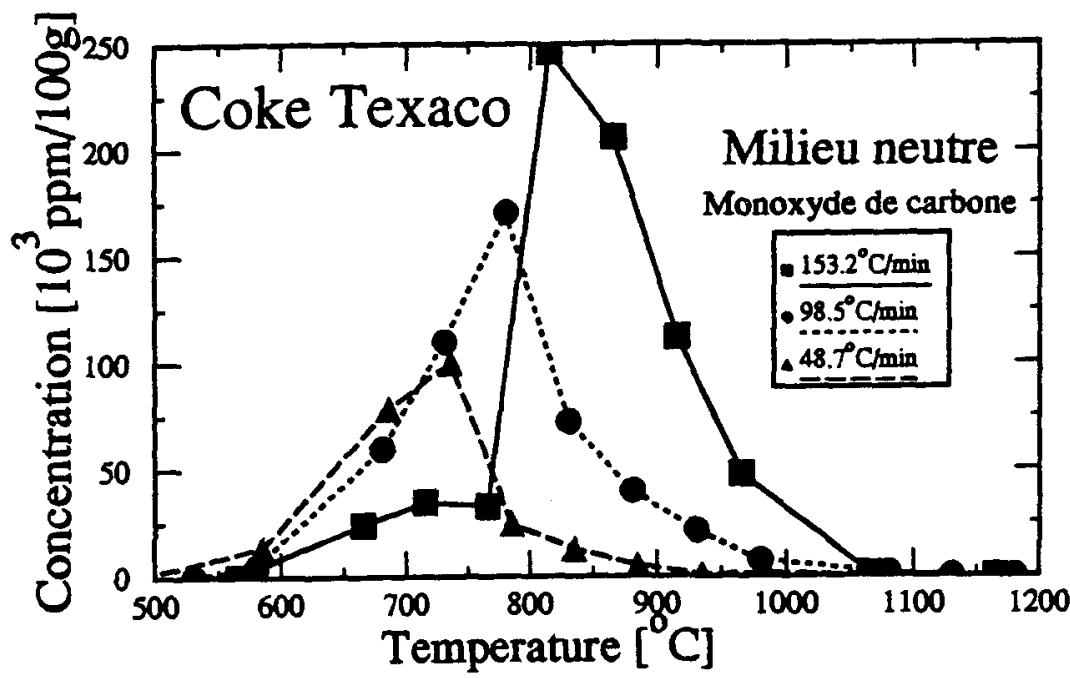

Figure 5.15: Effet du taux de chauffage sur le monoxyde de carbone du coke TEXACO, en milieu neutre ( $100 \%$ azote). 


\section{V.6 Modèle cinétique de dévolatilisation du coke vert en milieu neutre}

Un modèle similaire à celui de Wallouch et al. est utilisé pour interpréter les résultats expérimentaux en milieu neutre $[3,5,6,19]$. Les paramètres cinétiques sont déterminés pour chacun des produits volatils émis $\left(\mathrm{CH}_{4}, \mathrm{H}_{2}\right.$ et condensables).

Les résultats thermogravimétriques sont analysés par la méthode différentielle. La linéarisation de l'équation générale de la vitesse donne l'expression suivante:

$$
\begin{array}{cll}
\ln \left[\left(\mathrm{dX}_{\mathrm{i}} / \mathrm{dT}\right) /\left(1-\mathrm{X}_{\mathrm{i}}\right)^{\mathrm{n}}\right]=\ln (\mathrm{A} / \mathrm{c})-\mathrm{E} /(\mathrm{RT}) & (\mathrm{V} .1) \\
\mathrm{X}_{\mathrm{i}} & : \quad \begin{array}{l}
\text { fraction massique des matières volatiles dégagées } \\
\text { (conversion) }
\end{array} \\
\mathrm{n} & : \quad \text { ordre de la réaction } \\
\mathrm{A} & : & \text { facteur pré-exponentiel }\left[\mathrm{s}^{-1}\right] \\
\mathrm{C} & : & \text { vitesse de chauffage }\left[{ }^{\circ} \mathrm{C} / \mathrm{min}\right] \\
\mathrm{E} & : & \text { énergie d'activation }[\mathrm{J} / \mathrm{mol}] \\
\mathrm{R} & : & \text { constante des gaz parfaits }[\mathrm{J} / \mathrm{mol} \mathrm{K}]
\end{array}
$$

Les paramètres cinétiques sont déduits graphiquement. Si on porte en graphique $\ln \left[\left(\mathrm{dX}_{\mathrm{i}} / \mathrm{dT}\right) /\left(1-\mathrm{X}_{\mathrm{i}}\right)^{\mathrm{n}}\right]$ en fonction de $1 / \mathrm{T}$ pour différentes valeurs de $n$, l'ordre de la réaction est celui qui correspond à la meilleure droite obtenue, le facteur pré-exponentiel A est donné par l'ordonnée à l'origine de la droite et l'énergie d'activation $\mathrm{E}$ par la pente de la droite.

\section{V.6.1 Méthode de calcul}

1- Le pourcentage total de perte de poids de l'échantillon est calculé en divisant la perte de poids enregistrée au cours de l'expérience de calcination (fréquence=la seconde) par le poids initial de l'échantillon et en multipliant par 100. Cela permet de rapporter tous les résultats à $100 \mathrm{~g}$ de coke vert. On obtient ainsi la courbe TGA expérimentale, exprimant l'évolution de la perte de poids en fonction du temps. 
2- On dérive la courbe TGA expérimentale en fonction du temps. La courbe obtenue est ajustée par un ajustement polynomial à l'aide du logiciel commercial TEMPLE GRAPH; elle portera le nom de DTGA. On obtient ainsi la courbe DTGA expérimentale et la courbe DTGA ajustée.

3- La courbe DTGA ajustée est intégrée pour obtenir la TGA ajustée; ceci afin de comparer les résultats thermogravimétriques issus de l'expérience (TGA expérimentale) avec la courbe issue de l'ajustement (TGA ajustée).

4- On convertit les gaz analysés par chromatographie en unité de poids, en supposant que l'échantillon gazeux prélevé provient d'un mélange homogène sous un débit total constant de 10.6 litres par minute (égal au débit d'entrée), en utilisant la formule suivante:

(Concentration X Débit X Masse molaire) / (Volume molaire)

$\begin{array}{lll}\text { Concentration } & : & \text { [ppm] } \\ \text { Débit des gaz } & : & 10.6[\mathrm{~L} / \mathrm{min}]\left(\mathrm{à} 0^{\circ} \mathrm{C} \text { et } 1 \mathrm{~atm}\right) \\ \text { Masse molaire } & : & {[\mathrm{g} / \mathrm{mol}]} \\ \text { Volume molaire } & : & 22.4[\mathrm{~L} / \mathrm{mol}]\end{array}$

On obtient ainsi les courbes instantanées pour les gaz $\mathrm{CH}_{4}$ et $\mathrm{H}_{2}$, exprimant la vitesse de perte de poids en g/s relative à chaque produit (rapportée à 100 grammes de coke vert).

5- La courbe instantanée pour le goudron s'obtient en soustrayant les deux courbes instantanées de $\mathrm{l}^{\prime} \mathrm{H}_{2}$ et du $\mathrm{CH}_{4}$ de la courbe DTGA ajustée trouvée en 2. Le goudron représente ici tous les produits volatils émis, exceptés le $\mathrm{CH}_{4}$ et $\mathrm{l}^{\prime} \mathrm{H}_{2}$.

6- Les courbes instantanées sont intégrées afin d'obtenir les valeurs cumulatives de perte de poids pour les trois composants en fonction du temps. Les courbes instantanées peuvent également être tracées en fonction de la température. 
7- On calcule ensuite les conversions expérimentales pour $1^{\prime} \mathrm{H}_{2}, \mathrm{le}$ $\mathrm{CH}_{4}$ et le goudron en normalisant la courbe de perte de poids trouvée en 6 , en divisant la perte de poids instantanée par la perte de poids totale observée en fin d'expérience à $1200^{\circ} \mathrm{C}$. La conversion vaut $1(X=1)$ à la fin de l'expérience et 0 au début $(X=0)$. On obtient ainsi les conversions expérimentales.

8- Les paramètres cinétiques du modèle (A et $E$ ) sont déduits graphiquement à partir des conversions expérimentales pour les trois composants, en portant en graphique In $\left[\left(\mathrm{dX}_{\mathrm{i}} / \mathrm{dT}\right) /\left(1-\mathrm{X}_{\mathrm{i}}\right)^{\mathrm{n}}\right]$ en fonction de $1 / T$. L'ordre de la réaction est celui qui correspond à la meilleure droite (meilleure corrélation possible); la pente de la droite donne l'énergie d'activation $E$ et l'ordonnée à l'origine le facteur pré-exponentiel $A$. Afin de ne pas représenter la dévolatilisation par un mécanisme trop complexe dans le modèle, l'ordre de la réaction varie de 0.5 à 4 , par pas de 0.5 . La valeur de l'ordre qui donne la meilleure corrélation fixe l'ordre pour le calcul des paramètres E. et A. Dans notre cas, la corrélation fluctue entre 92 et $99.9 \%$.

9- Une fois les paramètres cinétiques connus pour les trois composants, ceux-ci sont introduits dans l'équation du modèle cinétique:

$$
\mathrm{dX}_{\mathrm{i}} / \mathrm{dt}=\mathrm{A}_{\mathrm{i}} \exp ^{\left(-\mathrm{E}_{1} / \mathrm{RT}\right)}\left(1-\mathrm{X}_{\mathrm{i}}\right)^{\mathrm{n} !}
$$

En procédant ensuite à une intégration numérique, on obtient les conversions issues du modèle. On peut ainsi juger de la pertinence du modèle proposé pour les trois composants.

\section{V.6.2 Ilustration de la méthode de calcul sur un exemple}

Afin d'illustrer la méthode de calcul détaillée dans le paragraphe précédant, prenons un exemple: le coke TEXACO à $150^{\circ} \mathrm{C} / \mathrm{min}$.

- Les figures 5.16 et 5.17 comparent les TGA et DTGA, issues des données expérimentales et de l'ajustement. On constate que les données expérimentales sont bien représentées par la courbe ajustée. 
- La figure 5.18 montre les courbes instantanées de perte de poids par unité de temps pour les gaz $\mathrm{H}_{2}, \mathrm{CH}_{4}$ et les condensables ainsi que la DTGA totale ajustée, en g/s. Ces valeurs sont rapportées à 100 grammes de coke vert. La courbe dérivée pour les condensables est obtenue par soustraction des deux courbes $\mathrm{CH}_{4}$ et $\mathrm{H}_{2}$ de la courbe totale. En annexe II, on trouve les courbes relatives aux deux autres taux de chauffage pour le même type de coke.

- La figure 5.19 montre les courbes cumulatives de perte de poids en fonction de la température pour les trois composants. Elles sont obtenues par intégration des courbes instantanées.

Le tableau 5.2 présente la perte de poids totale due au départ des trois composants: $\mathrm{H}_{2}, \mathrm{CH}_{4}$ et condensables, pour les trois taux de chauffage et les quatre cokes. La différence entre la perte de poids totale et la somme des pertes de poids dues aux trois composants provient de l'humidité contenue dans l'échantillon de coke vert (environ 1 à $2 \%$ ).

- Les figures 5.20 à 5.22 montrent l'influence du taux de chauffage sur la courbe cumulative de perte de poids pour les trois composants respectivement.

D'une manière générale, un taux de chauffage élevé retarde le départ des produits volatils légers de type hydrogène et méthane, comme déjà observé antérieurement par d'autres auteurs $[3,6,22]$.

La quantité d'hydrogène émise augmente avec la diminution du taux de chauffage durant toute la durée de la calcination pour le coke TEXACO (figure 5.20) ou jusqu'à $950-1000^{\circ} \mathrm{C}$ pour les trois autres cokes. En effet, poür les cokes RTW, CONOCO et YPF, la quantité totale maximale d' $\mathrm{H}_{2}$ est observée à $100^{\circ} \mathrm{C} / \mathrm{min}$ (tableau 5.2 ).

La quantité de méthane émise augmente avec la diminution du taux de chauffage jusqu'à $800-850^{\circ} \mathrm{C}$ pour les cokes TEXACO (figure 5.21), RTW et CONOCO; et jusqu'à $750^{\circ} \mathrm{C}$ pour le coke YPF. En effet, la quantité totale maximale de $\mathrm{CH}_{4}$ est observée à 100 ou $150^{\circ} \mathrm{C} / \mathrm{min}$ (tableau 5.2). 
En ce qui concerne les condensables, on remarque qu'ils suivent la même évolution jusqu'à $650^{\circ} \mathrm{C}$ quel que soit le taux de chauffage; ensuite la quantité cumulative de condensables augmente avec le taux de chauffage pour le coke TEXACO (figure 6.22). Dans le tableau 5.2, on constate que la quantité totale maximale de condensables est également observée à $150^{\circ} \mathrm{C} / \mathrm{min}$ pour les cokes YPF et CONOCO; par contre pour le coke RTW elle est observée à $100^{\circ} \mathrm{C} / \mathrm{min}$.

- Le tableau 5.3 donne les paramètres cinétiques des trois composés (hydrogène, méthane et condensables) pour les trois taux de chauffage et les quatre types de cokes. L'ordre de la réaction est fixé à 1.5 ou 2 pour le méthane et l'hydrogène; il est fixé à 1 ou 2 pour les condensables. Les figures 5.23 à 5.25 comparent les conversions expérimentales issues de l'expérience avec les conversions calculées d'après le modèle cinétique, pour les trois composants: hydrogène, méthane et condensables. Les conversions obtenues pour le coke TEXACO aux deux autres taux de chauffage sont données en annexe III.

On remarque, au vu des figures 5.23 à 5.25, que les données expérimentales sont bien représentées par le modèle cinétique proposé ( $r>99.2 \%$ ) pour le coke TEXACO à $150^{\circ} \mathrm{C} / \mathrm{min}$. La comparaison de l'expérience avec le modèle est donnée en annexe III pour les deux autres taux de chauffage.

D'une manière générale, on constate que le modèle de dévolatilisation proposé pour l'hydrogène et le méthane est bien représenté par les résultats expérimentaux et ce pour les quatre types de coke. Un coefficient de corrélation fluctuant entre 92.8 et $99.9 \%$ en est la preuve (tableau 5.3). Par contre, pour les condensables, le modèle reflète moins bien les résultats expérimentaux et ce pour les quatre cokes. Rappelons à ce sujet que les condensables représentent, dans le cadre de ce travail, tous les produits volatils autres que l'hydrogène et le méthane. Cette généralisation du terme "condensables" peut expliquer la moins bonne corrélation entre les résultats expérimentaux (obtenus par soustraction des courbes relatives à $l^{\prime} \mathrm{H}_{2}$ et au $\mathrm{CH}_{4}$ de la courbe totale) et le modèle cinétique. 
Temperature $\left[{ }^{\circ} \mathrm{C}\right]$

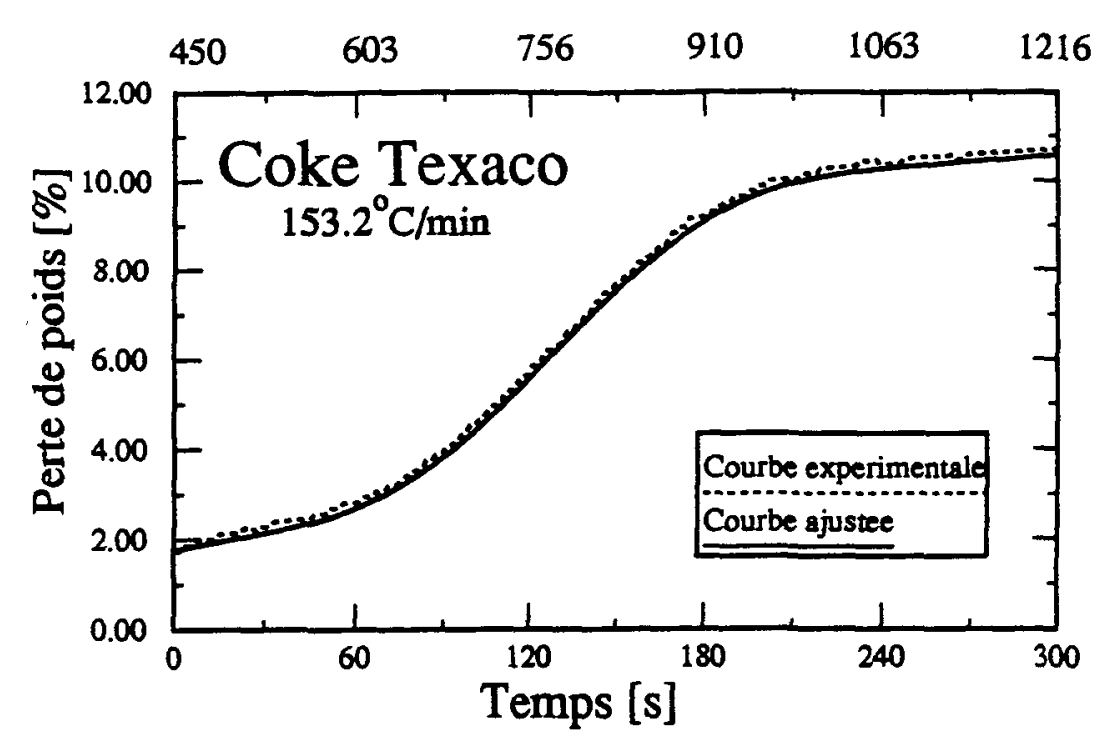

Figure 5.16: Comparaison des courbes ajustée et expérimentale pour le coke TEXACO à $150^{\circ} \mathrm{C} / \mathrm{min}$, en milieu neutre ( $100 \%$ azote).

Temperature $\left[{ }^{\circ} \mathrm{C}\right]$

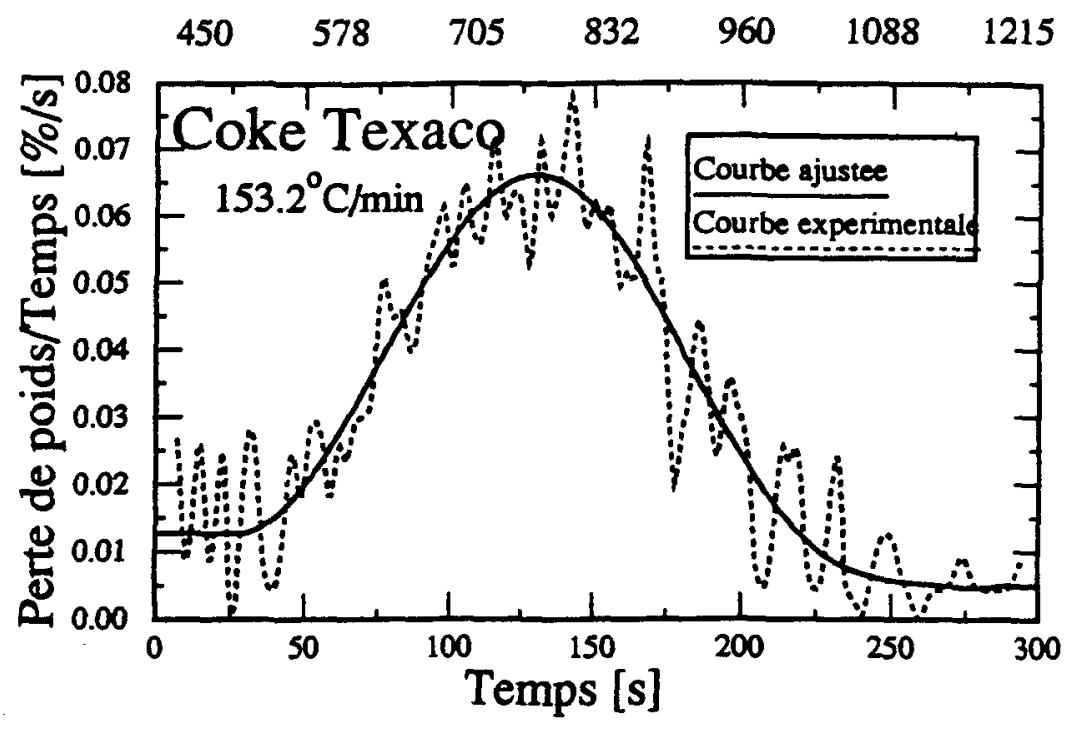

Figure 5.17 : Comparaison des dérivées des courbes expérimentale et ajustée du coke TEXACO à $150^{\circ} \mathrm{C} / \mathrm{min}$, en milieu neutre. 


\section{Temperature $\left[{ }^{\circ} \mathrm{C}\right]$}

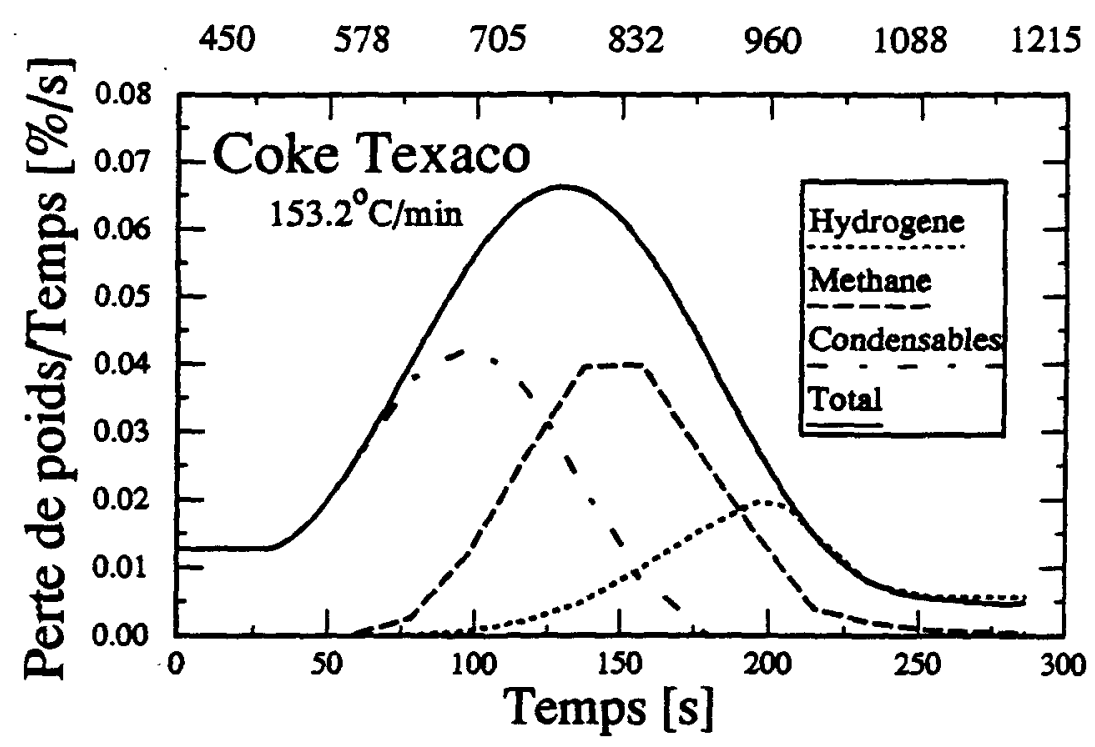

Figure 5.18: Valeurs instantanées pour le coke TEXACO à $150^{\circ} \mathrm{C} / \mathrm{min}$, en milieu neutre (100\% azote).

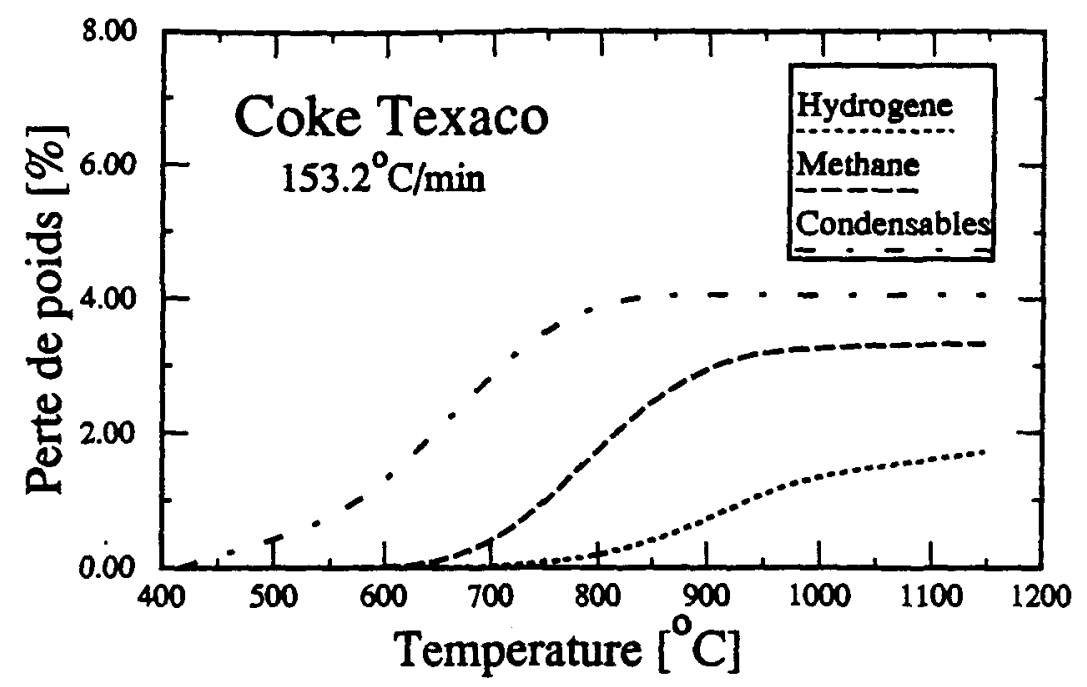

Figure 5.19: Valeurs cumulatives pour le coke TEXACO à $150^{\circ} \mathrm{C} / \mathrm{min}$, en milieu neutre ( $100 \%$ azote). 


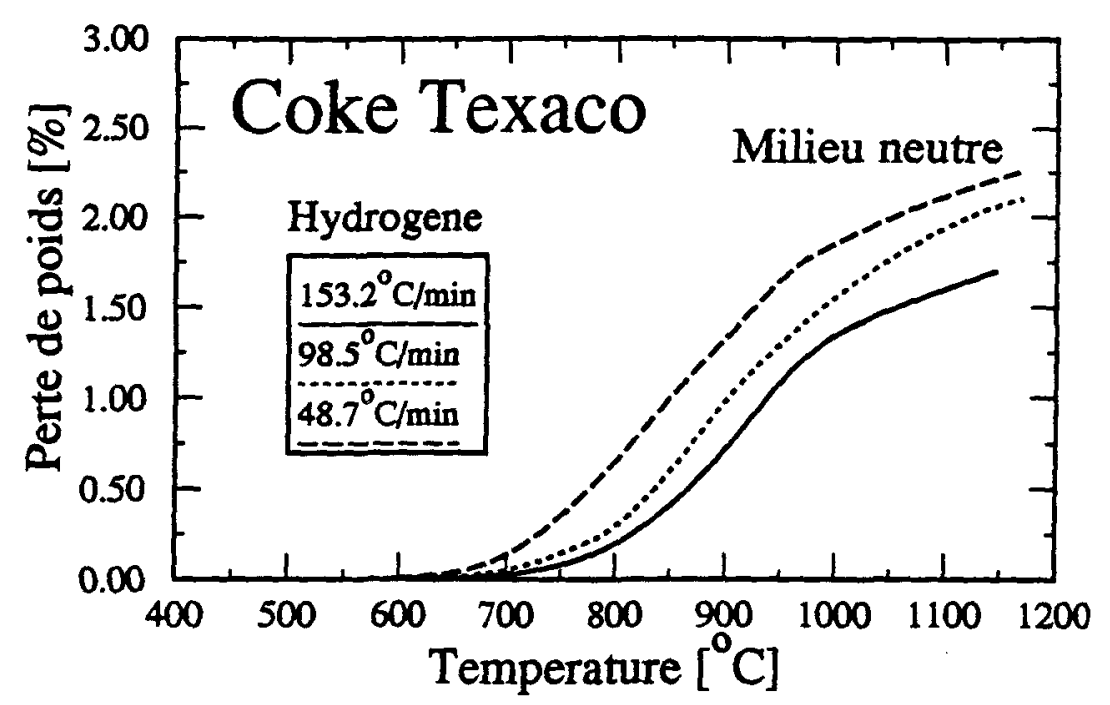

Figure 5.20 : Effet du taux de chauffage sur l'émission d'hydrogène pour le coke TEXACO, en milieu neutre ( $100 \%$ azote).

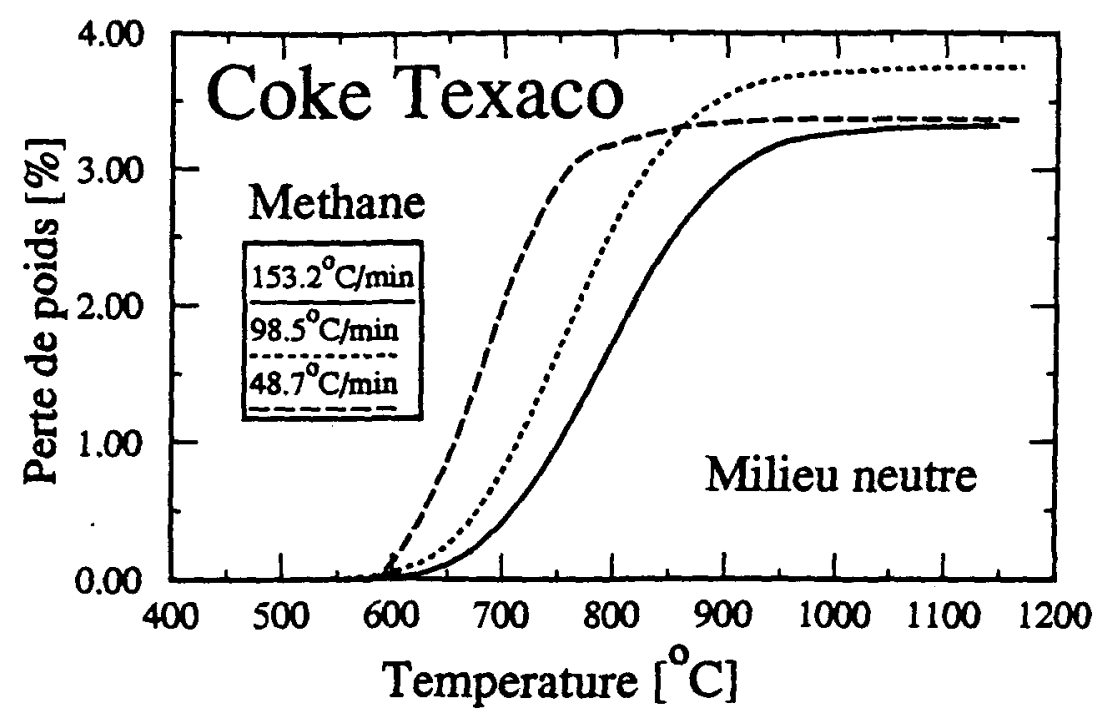

Figure 5.21 : Effet du taux de chauffage sur l'émission de méthane pour le coke TEXACO, en milieu neutre (100\% azote). 


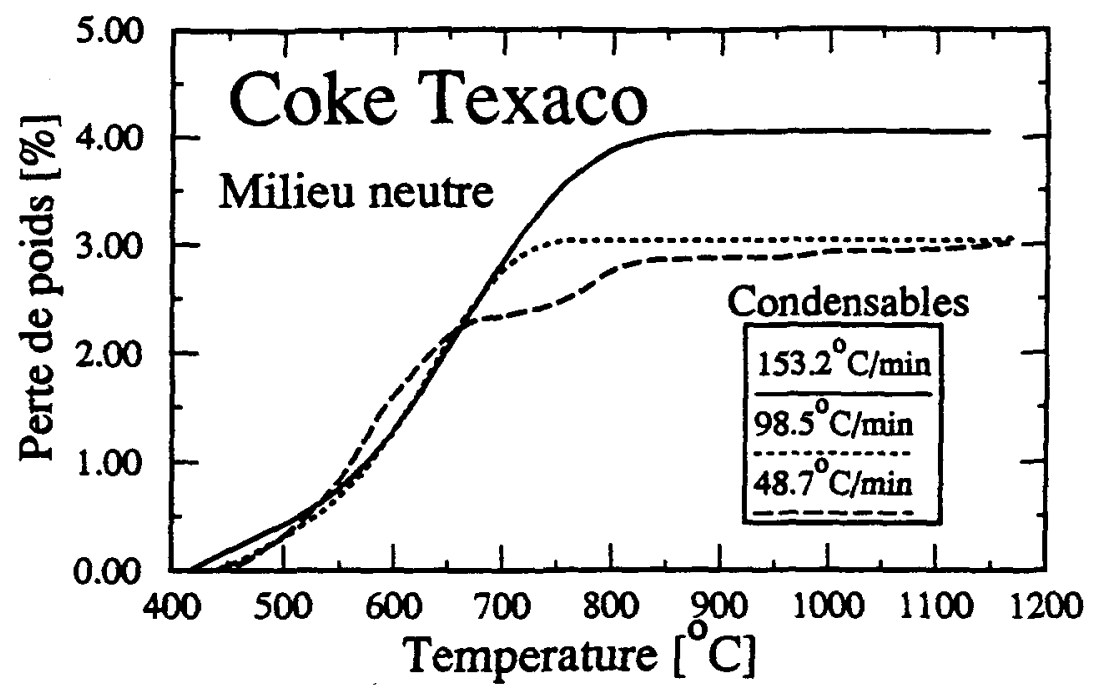

Figure 5.22 : Effet du taux de chauffage sur l'émission des condensables pour le coke TEXACO, en milieu neutre ( $100 \%$ azote).

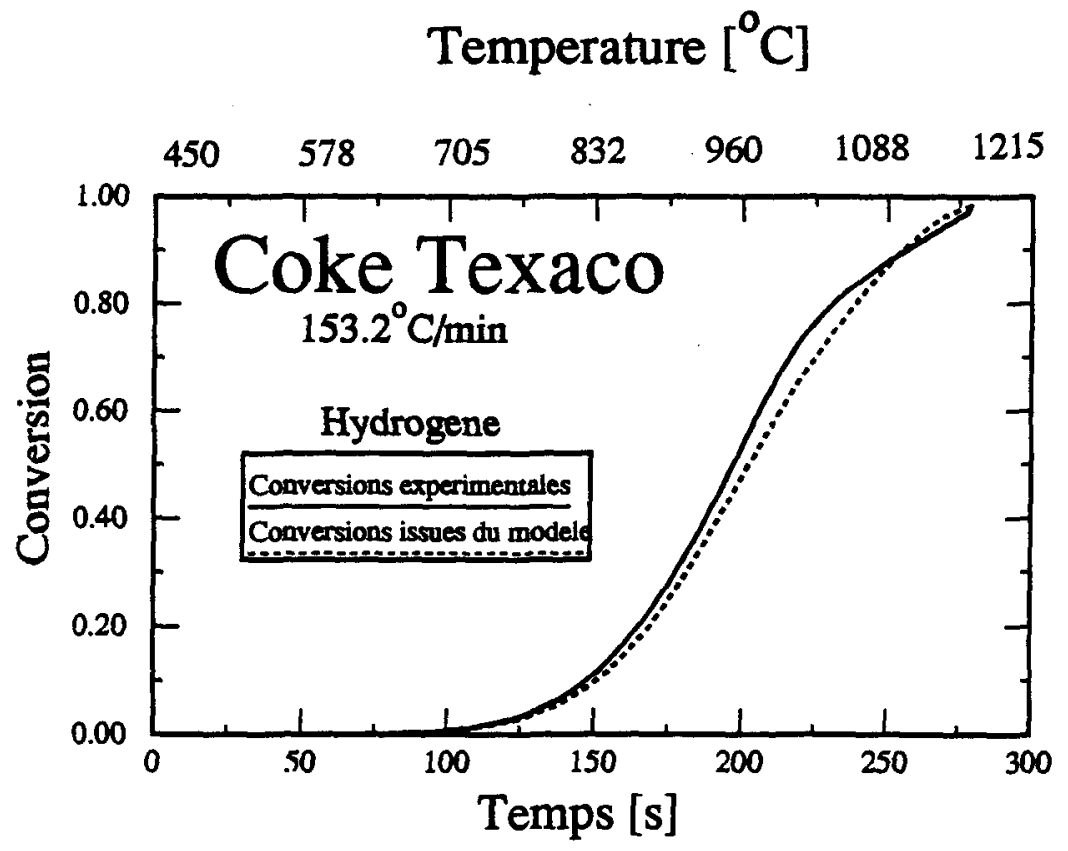

Figure 5.23 : Conversions pour l'hydrogène du coke TEXACO à $150^{\circ} \mathrm{C} / \mathrm{min}$, en milleu neutre ( $100 \%$ azote). 


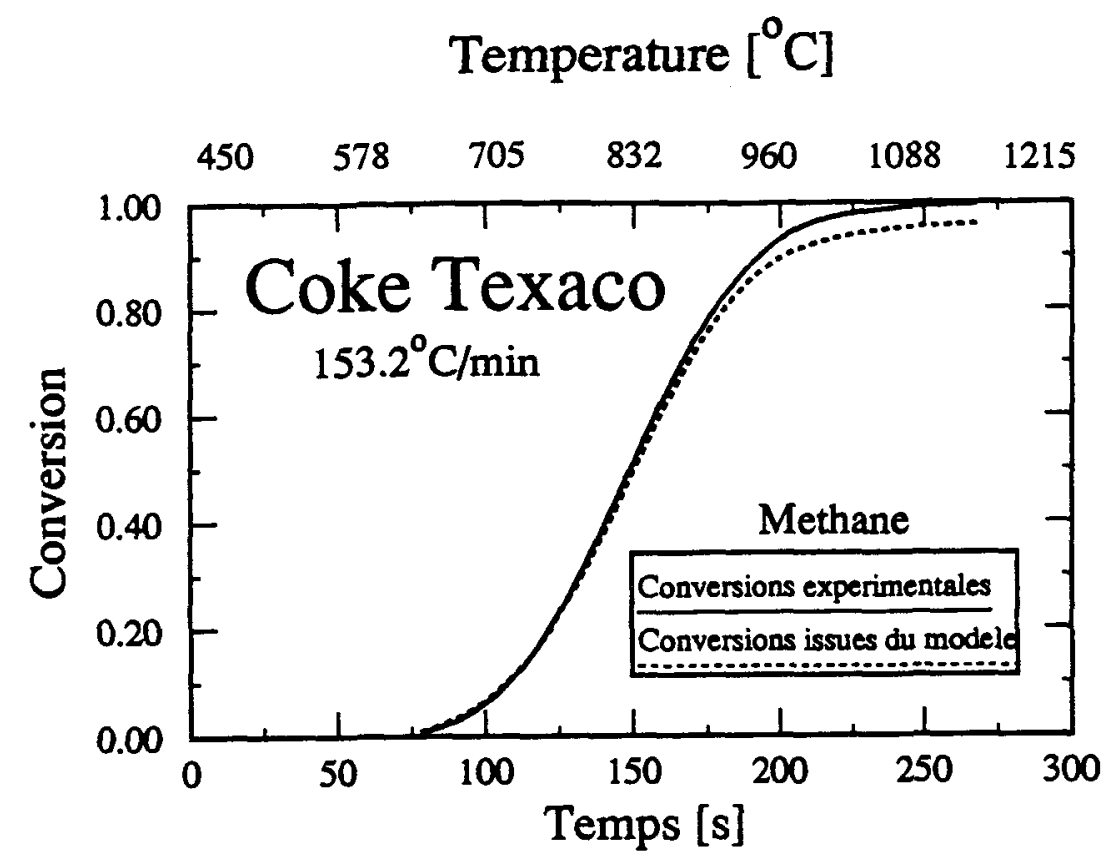

Figure 5.24: Conversions pour le méthane du coke TEXACO à $150^{\circ} \mathrm{C} / \mathrm{min}$, en milieu neutre (100\% azote).

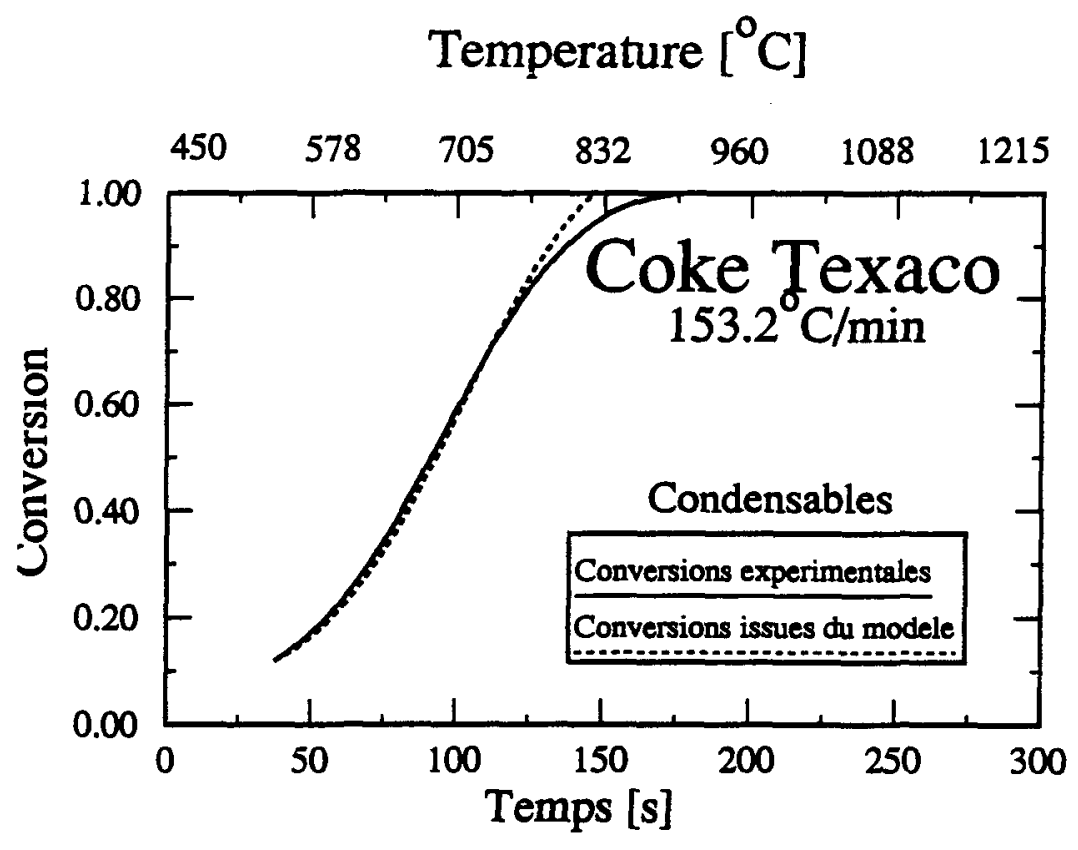

Figure 5.25 : Conversions pour les condensables du coke TEXACO à $150^{\circ} \mathrm{C} / \mathrm{min}$, en milieu neutre $(100 \%$ azote $)$. 
Tableau 5.2: $\quad$ Perte de poids totale $\left(\mathrm{a} 1200^{\circ} \mathrm{C}\right)$ relative à chaque produit en milieu neutre $\left(100 \% \mathrm{~N}_{2}\right)$.

\begin{tabular}{|c|c|c|c|c|c|}
\hline Cokes & $\begin{array}{l}\text { Taux de } \\
\text { chauffage } \\
\left.\text { [ }{ }^{\circ} \mathrm{C} / \mathrm{min}\right]\end{array}$ & $\begin{array}{l}\text { Hydrogène } \\
{[\%]}\end{array}$ & $\begin{array}{l}\text { Méthane } \\
{[\%]}\end{array}$ & $\begin{array}{c}\text { Condensables } \\
{[\%]}\end{array}$ & $\begin{array}{c}\text { Total } \\
{[\%]}\end{array}$ \\
\hline TEXACO & $\begin{array}{r}153.2 \\
98.5 \\
48.7\end{array}$ & $\begin{array}{l}1.70 \\
2.10 \\
2.25\end{array}$ & $\begin{array}{l}3.32 \\
3.75 \\
3.37\end{array}$ & $\begin{array}{l}4.05 \\
3.05 \\
3.01\end{array}$ & $\begin{array}{r}10.57 \\
9.77 \\
10.40\end{array}$ \\
\hline RTW & $\begin{array}{r}147.5 \\
100.8 \\
49.7\end{array}$ & $\begin{array}{l}2.11 \\
2.34 \\
2.32\end{array}$ & $\begin{array}{l}3.43 \\
3.40 \\
3.26\end{array}$ & $\begin{array}{l}3.05 \\
3.10 \\
2.59\end{array}$ & $\begin{array}{l}10.20 \\
10.30 \\
10.11\end{array}$ \\
\hline YPF & $\begin{array}{r}150.6 \\
97.2 \\
48.0\end{array}$ & $\begin{array}{l}2.06 \\
2.50 \\
2.10\end{array}$ & $\begin{array}{l}3.71 \\
4.30 \\
3.09\end{array}$ & $\begin{array}{l}5.30 \\
3.42 \\
4.54\end{array}$ & $\begin{array}{l}12.65 \\
11.82 \\
11.83\end{array}$ \\
\hline conoco & $\begin{array}{r}147.2 \\
97.4 \\
47.8\end{array}$ & $\begin{array}{l}2.10 \\
2.14 \\
2.00\end{array}$ & $\begin{array}{l}2.86 \\
2.85 \\
2.14\end{array}$ & $\begin{array}{l}3.19 \\
2.40 \\
2.94\end{array}$ & $\begin{array}{l}8.89 \\
8.6 \\
8.52\end{array}$ \\
\hline
\end{tabular}


Tableau 5.3: $\quad$ Paramètres cinétiques relatifs à chaque produit en milieu neutre $\left(100 \% \mathrm{~N}_{2}\right)$, pour les quatres types de cokes: TEXACO (a), RTW (b),YPF (c) et CONOCO (d).

\begin{tabular}{|c|c|c|c|}
\hline \multicolumn{4}{|c|}{ COKE TEXACO } \\
\hline $\begin{array}{l}\text { Taux de } \\
\text { chauffage } \\
{\left[{ }^{\circ} \mathrm{C} / \mathrm{min}\right]}\end{array}$ & $\begin{array}{c}\text { Hydrogène } \\
E\left[\mathrm{~J} / \mathrm{moll}^{-1}\right] \\
\mathrm{A}\left[\mathrm{s}^{-1}\right]\end{array}$ & Méthane & Condensables \\
\hline 153.2 & $\begin{array}{l}\mathrm{n}=1.5 \\
\mathrm{E}=154607 \\
\mathrm{~A}=163344 \\
\mathrm{r}=99.3\end{array}$ & $\begin{array}{l}n=1.5 \\
E=134335 \\
A=121406 \\
r=99.8\end{array}$ & $\begin{array}{l}n=1 \\
E=81073 \\
A=832 \\
r=99.2\end{array}$ \\
\hline 98.5 & $\begin{array}{l}n=2 \\
E=163250 \\
A=390264 \\
r=99.2\end{array}$ & $\begin{array}{l}n=2 \\
E=185872 \\
A=9.7 \quad 10^{7} \\
r=99.7\end{array}$ & $\begin{array}{l}n=1 \\
E=83029 \\
A=1351 \\
r=98.6\end{array}$ \\
\hline 48.7 & $\begin{array}{l}n=2 \\
E=133574 \\
A=14611 \\
r=99\end{array}$ & $\begin{array}{l}n=2 \\
E=246246 \\
A=5.310^{11} \\
r=92.8\end{array}$ & $\begin{array}{l}n=2 \\
E=58563 \\
A=31 \\
r=78\end{array}$ \\
\hline
\end{tabular}

n : $\quad$ Ordre de la réaction

E : Energie d'activation $[\mathrm{J} / \mathrm{mol}]$

A : $\quad$ Facteur pré-exponentiel $\left[\mathrm{s}^{-1}\right]$

r : Coefficient de corrélation modèle-expérience [\%] 


\begin{tabular}{|c|c|c|c|}
\hline \multicolumn{4}{|c|}{ COKE RTW } \\
\hline $\begin{array}{l}\text { Taux de } \\
\text { chauffage } \\
{\left[^{\circ} \mathrm{C} / \mathrm{min}\right]}\end{array}$ & $\begin{array}{c}\text { Hydrogène } \\
\mathrm{E}[\mathrm{J} / \mathrm{mole}] \\
\mathrm{A}\left[\mathrm{s}^{-1}\right]\end{array}$ & Méthane & Condensables \\
\hline 147.5 & $\begin{array}{l}n=2 \\
E=156504 \\
A=368819 \\
r=99.1\end{array}$ & $\begin{array}{l}n=2 \\
E=192181 \\
A=4.34 \quad 10^{8} \\
r=99.6\end{array}$ & $\begin{array}{l}\mathrm{n}=1 \\
\mathrm{E}=70864 \\
\mathrm{~A}=449 \\
\mathrm{r}=94.3\end{array}$ \\
\hline 100.8 & $\begin{array}{l}n=2 \\
E=146365 \\
A=72239 \\
r=99.5\end{array}$ & $\begin{array}{l}n=1.5 \\
E=145938 \\
A=873216 \\
r=99.4\end{array}$ & $\begin{array}{l}n=2 \\
E=127013 \\
A=2.07 \quad 10^{6} \\
r=88.9\end{array}$ \\
\hline 49.7 & $\begin{array}{l}n=1.5 \\
E=94983 \\
A=137 \\
r=97.9\end{array}$ & $\begin{array}{l}\mathrm{n}=1.5 \\
\mathrm{E}=184263 \\
\mathrm{~A}=1.59610^{8} \\
\mathrm{r}=94.1\end{array}$ & $\begin{array}{l}n=2 \\
E=104464 \\
A=46743 \\
r=87.7\end{array}$ \\
\hline
\end{tabular}

\begin{tabular}{|c|c|c|c|}
\hline c) & $\mathrm{COI}$ & & \\
\hline $\begin{array}{l}\text { Taux de } \\
\text { chauffage } \\
{\left[{ }^{\circ} \mathrm{C} / \mathrm{min}\right]}\end{array}$ & $\begin{array}{c}\text { Hydrogène } \\
\mathrm{E}\left[\mathrm{J} / \mathrm{moll}^{-1}\right. \\
\left.\mathrm{A}^{-1} \mathrm{~s}^{-1}\right]\end{array}$ & Méthane & Condensables \\
\hline 150.6 & $\begin{array}{l}n=2 \\
E=156711 \\
A=203932 \\
r=99.8\end{array}$ & $\begin{array}{l}n=1.5 \\
E=140169 \\
A=255886 \\
r=99.9\end{array}$ & $\begin{array}{l}n=2 \\
E=123507 \\
A=1.154 \quad 10^{6} \\
r=91.3\end{array}$ \\
\hline 97.2 & $\begin{array}{l}n=2 \\
E=133899 \\
A=21421 \\
r=99.2\end{array}$ & $\begin{array}{l}\mathrm{n}=1.5 \\
\mathrm{E}=142285 \\
\mathrm{~A}=639532 \\
\mathrm{r}=98.5\end{array}$ & $\begin{array}{l}n=2 \\
E=109627 \\
A=264075 \\
r=85.1\end{array}$ \\
\hline 48.0 & $\begin{array}{l}n=2 \\
E=135041 \\
A=17276 \\
r=98.4\end{array}$ & $\begin{array}{l}n=1.5 \\
E=196561 \\
A=6.43410^{8} \\
r=94.7\end{array}$ & $\begin{array}{l}n=2 \\
E=49346 \\
A=13 \\
r=67.7\end{array}$ \\
\hline
\end{tabular}




\begin{tabular}{|c|c|c|c|}
\hline \multicolumn{4}{|c|}{ COKE CONOCO } \\
\hline $\begin{array}{l}\text { Taux de } \\
\text { chauffage } \\
{\left[{ }^{\circ} \mathrm{C} / \mathrm{min}\right]}\end{array}$ & $\begin{array}{c}\text { Hydrogène } \\
\text { E }\left[\mathrm{J} / \mathrm{mol}^{-1}\right] \\
\mathrm{A}\left[\mathrm{s}^{-1}\right]\end{array}$ & Méthane & Condensables \\
\hline 147.2 & $\begin{array}{l}n=2 \\
E=173896 \\
A=1.148 \quad 10^{6} \\
r=99.2\end{array}$ & $\begin{array}{l}\mathrm{n}=1.5 \\
\mathrm{E}=163936 \\
\mathrm{~A}=2.783 \quad 10^{6} \\
\mathrm{r}=99.2\end{array}$ & $\begin{array}{l}n=1 \\
E=63046 \\
A=91 \\
r=90.9\end{array}$ \\
\hline 97.4 & $\begin{array}{l}n=2 \\
E=163521 \\
A=392691 \\
r=98.9\end{array}$ & $\begin{array}{l}n=1.5 \\
E=171555 \\
A=1.076 \quad 10^{7} \\
r=99.4\end{array}$ & $\begin{array}{l}n=2 \\
E=99797 \\
A=24154 \\
r=89.5\end{array}$ \\
\hline 47.8 & $\begin{array}{l}n=1.5 \\
E=111011 \\
A=817 \\
r=97.8\end{array}$ & $\begin{array}{l}n=1.5 \\
E=199194 \\
A=7.84510^{8} \\
r=94.7\end{array}$ & $\begin{array}{l}n=2 \\
E=103397 \\
A=15381 \\
r=93.8\end{array}$ \\
\hline
\end{tabular}

\section{V.7 Conclusions}

L'expérience démontre que le changement de granulométrie du coke vert de $-20+35$ mailles $(0.42-0.83 \mathrm{~mm})$ à $-8+10$ mailles $(1.65-2.36 \mathrm{~mm}) \mathrm{n}$ 'a pas d'influence sur la cinétique de dévolatilisation du coke au cours de sa calcination jusqu'à $1200^{\circ} \mathrm{C}$, et ce dans les conditions de travail précisées au paragraphe V.2.

Par contre, il n'en est pas de même pour la forme du creuset qui influence considérablement les résultats. Le creuset de type "ouvert" permet une évacuation plus aisée des produits volatils formés au cours du traitement thermique, comparativement au creuset de type "fermé" dans lequel la diffusion des volatils est plus difficile et plus lente [2,3]. Ainsi, l'analyse des gaz recueillis révèle des concentrations plus importantes avec le creuset de type "ouvert". 
Quant à la cinétique de dévolatilisation du coke vert en atmosphère neutre d'azote, le modèle cinétique de Wallouch appliqué à l'hydrogène et au méthane représente bien les résultats expérimentaux $[2,3,6,19]$. Cependant, le modèle cinétique est un peu moins fidèle en ce qui concerne les produits condensables (surtout à $50^{\circ} \mathrm{C} / \mathrm{min}$ ). 


\section{PYROLYSE DU COKE VERT EN MILIEU OXYDANT}

\section{VI.1 Introduction}

Les conditions expērimentales des expériences menées en milieu oxydant sont les suivantes:

Creuset

Types de coke

Poids échantillon

Débit azote+air

Débit d'aspiration des gaz

$\%$ d'oxygène dans le milieu :

Taux de chauffage type "ouvert"

TEXACO, RTW, YPF et CONOCO $-8+10$ mailles $(1.65-2.36 \mathrm{~mm})$

$: \quad 10 \mathrm{~g}$

: $\quad 10.6 \mathrm{~L} / \mathrm{min}\left(\mathrm{à} 0^{\circ} \mathrm{C}\right.$ et $\left.1 \mathrm{~atm}\right)$

: $\quad 313 \mathrm{~mL} / \mathrm{min}$

$3 \%, 5 \%, 7 \%, 10 \%$

$150^{\circ} \mathrm{C} / \mathrm{min}, 100^{\circ} \mathrm{C} / \mathrm{min}, 50^{\circ} \mathrm{C} / \mathrm{min}$

La référence est le milieu constitué d'un mélange d'air et d'azote dans les proportions volumiques respectives de $25 \%$ d'air et $75 \%$ d'azote, ce qui correspond à un pourcentage d'oxygène de $5 \%$. Le choix d'un milieu oxydant à $5 \%$ d'oxygène est le résultat d'un compromis entre un pourcentage suffisamment élevé pour assurer la combustion des volatils et du coke, et un pourcentage d'une "agressivité" acceptable pour permettre une durée de vie raisonnable des creusets au cours des expériences.

Les résultats thermogravimétriques et l'analyse des gaz font l'objet des paragraphes VI.2 à VI.4. La comparaison des thermogrammes en milieux neutre et oxydant est donnée au paragraphe VI.2.3 pour le coke TEXACO; 
l'annexe IV présente les thermogrammes pour les trois autres types de coke. Le paragraphe VI.5 donne les vitesses instantanées pour le coke TEXACO; les trois autres cokes sont présentés en annexe V. Le paragraphe V.6 étudie l'influence de la pression partielle en oxygène dans le milieu sur la calcination du coke TEXACO à $150^{\circ} \mathrm{C} / \mathrm{min}$. Enfin, le chapitre VI.7 est consacré aux calculs d'équilibre pour juger de la cinétique de combustion des matières volatiles au cours de la calcination du coke.

\section{VI.2 Résultats thermogravimétriques}

\section{VI.2.1 Comparaison des cokes}

La figure 6.1 compare la courbe thermogravimêtrique expérimentale issue des données expérimentales de perte de poids enregistrées en fonction de la température au cours de l'expérience de pyrolyse, avec la courbe thermogravimétrique ajustée issue de l'ajustement polynomial de la courbe expérimentale, pour le coke TEXACO, aux trois taux de chauffage. On constate que les données expérimentales sont bien représentées par les courbes ajustées et ce aux trois taux de chauffage. Les résultats qui suivent sont données en fonction des courbes ajustées.

Les figures 6.2 à 6.4 comparent les courbes thermogravimétriques de perte de poids obtenues pour les quatres types de coke, et ce pour les trois taux de chauffage respectivement. Au regard de ces figures, on peut faire les mêmes constatations qu'en milieu neutre, à savoir que la perte de poids maximale est observée pour le coke YPF qui contient le plus haut pourcentage de matières volatiles. La perte de poids minimale est observée pour le coke CONOCO; alors que les cokes RTW et TEXACO contenant des pourcentages très proches en matières volatiles suivent la même tendance. Le tableau 6.1 donne la perte de poids totale observée en fin de calcination aux trois taux de chauffage et ce pour les quatre cokes. 


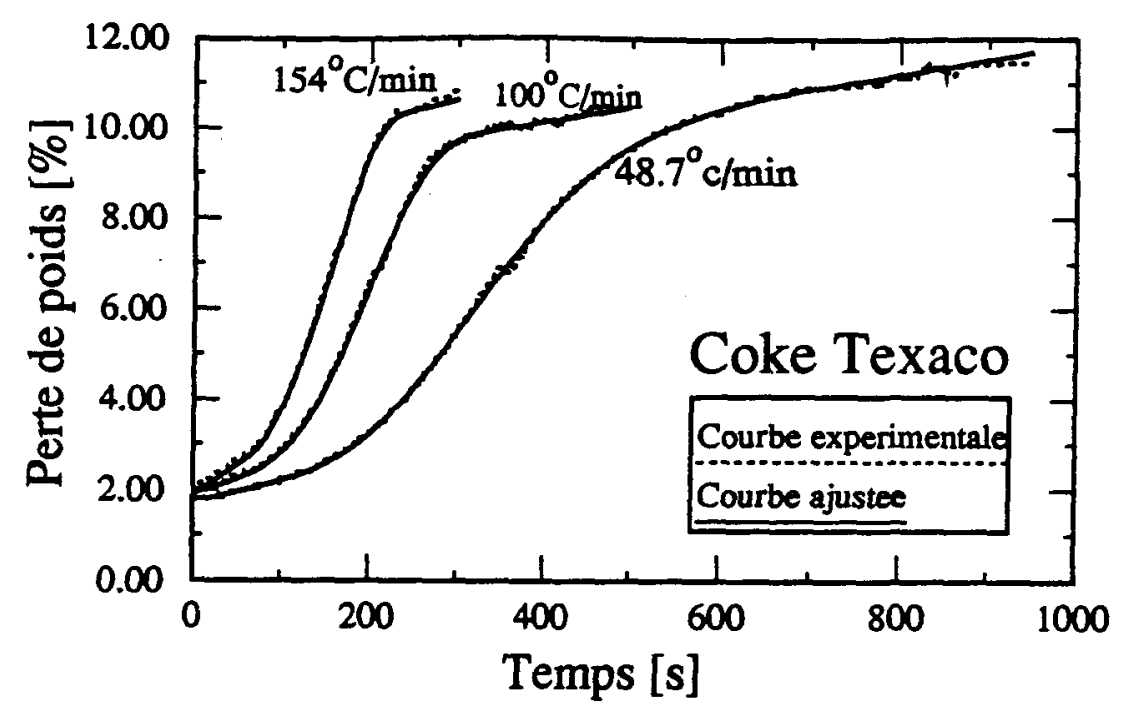

Figure 6.1: Comparaison des courbes expérimentale et ajustée pour le coke TEXACO, aux trois taux de chauffage en milieu oxydant (95\% azote, $5 \%$ oxygène).

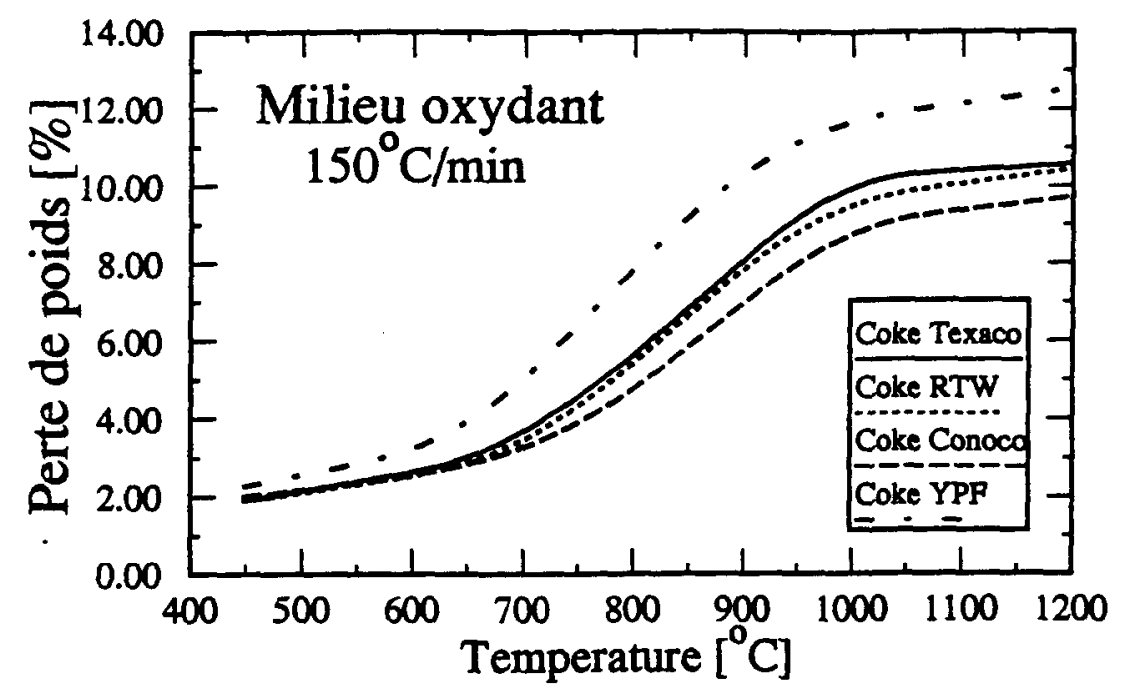

Figure 6.2 : Comparaison des thermogrammes à $150^{\circ} \mathrm{C} / \mathrm{min}$, en milieu oxydant ( $95 \%$ azote, $5 \%$ oxygène). 


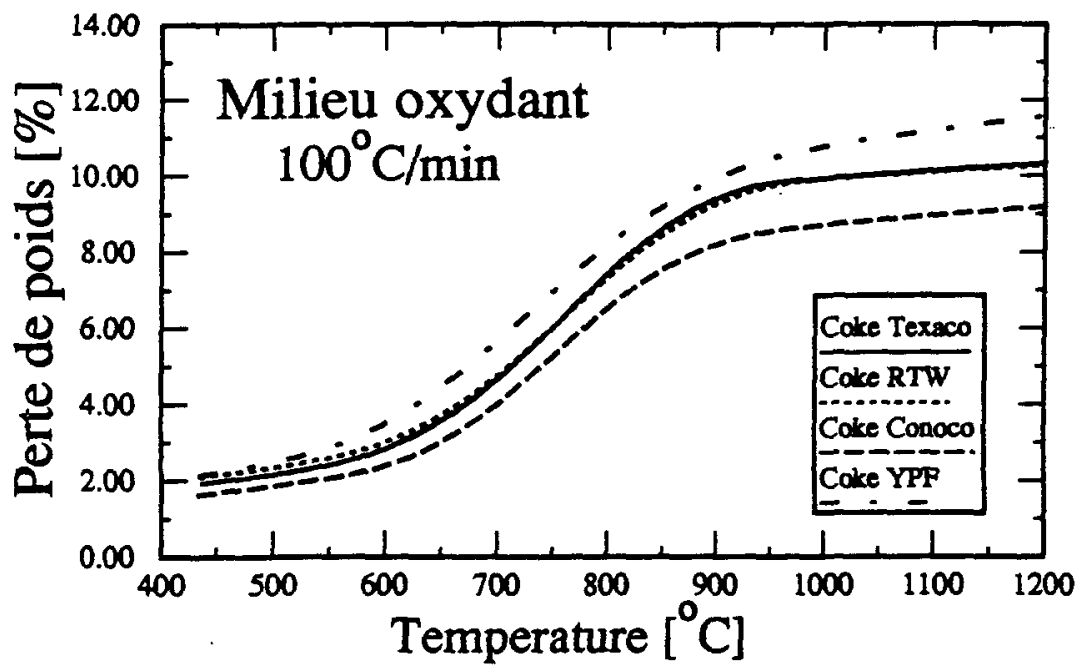

Figure 6.3: Comparaison des thermogrammes à $100^{\circ} \mathrm{C} / \mathrm{min}$, en milieu oxydant ( $95 \%$ azote, $5 \%$ oxygène).

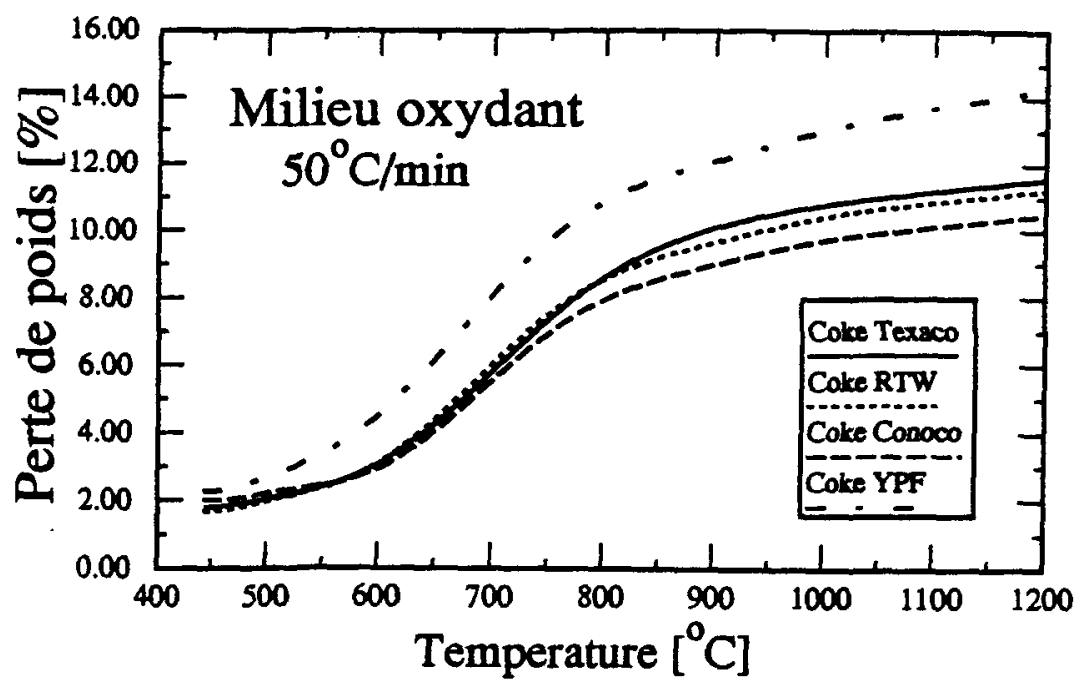

Figure 6.4: Comparaison des thermogrammes à $50^{\circ} \mathrm{C} / \mathrm{min}$, en milieu oxydant (95\% azote, $5 \%$ oxygène). 
Tableau 6.1: $\quad$ Perte de poids totale en fin de calcination $\left(1200^{\circ} \mathrm{C}\right)$ en milieu oxydant $\left(5 \% \mathrm{O}_{2}-95 \% \mathrm{~N}_{2}\right)$

\begin{tabular}{|c|c|c|}
\hline Cokes & $\begin{array}{l}\text { Taux de chauffage } \\
{\left[{ }^{\circ} \mathrm{C} / \mathrm{min}\right]}\end{array}$ & Perte $\underset{[\%]}{\text { de poids }}$ \\
\hline TEXACO & $\begin{array}{r}154.0 \\
100.0 \\
49.7\end{array}$ & $\begin{array}{l}10.60 \\
10.34 \\
11.60\end{array}$ \\
\hline RTW & $\begin{array}{r}158.0 \\
101.0 \\
48.2\end{array}$ & $\begin{array}{l}10.49 \\
10.29 \\
11.27\end{array}$ \\
\hline YPF & $\begin{array}{r}152.9 \\
101.0 \\
49.1\end{array}$ & $\begin{array}{l}12.53 \\
11.61 \\
14.31\end{array}$ \\
\hline CONOCO & $\begin{array}{r}156.7 \\
96.6 \\
47.9\end{array}$ & $\begin{array}{r}9.74 \\
9.20 \\
10.50\end{array}$ \\
\hline
\end{tabular}

\section{VI.2.2 Effet du taux de chauffage}

Les figures 6.5 à 6.8 montrent l'influence du taux de chauffage sur la courbe thermogravimétrique de perte de poids, et ce pour les quatre types de coke respectivement. Comme en milieu neutre, l'accroissement du taux de chauffage en milieu oxydant engendre un retard dans l'émission des produits volatils entre 500 et $900^{\circ} \mathrm{C}[3,22]$. Par contre, à plus haute température, on constate que la perte de poids augmente avec la diminution du taux de chauffage; et ce en raison des réactions de combustion du coke qui débutent à haute température lorsque le taux de chauffage est suffisamment faible (surtout $50^{\circ} \mathrm{C} / \mathrm{min}$ ). Dans le tableau 6.1 , on constate que la perte de poids totale observée en fin de calcination est maximale à $50^{\circ} \mathrm{C} / \mathrm{min}$, et ce pour les quatre types de coke. 
En milieu neutre, la courbe thermogravimétrique à $150^{\circ} \mathrm{C} / \mathrm{min}$ est la courbe caractéristique présentant un retard à basse température $\left(\mathrm{T}<900^{\circ} \mathrm{C}\right)$ et une perte de poids supérieure à haute température $\left(\mathrm{T}>900^{\circ} \mathrm{C}\right)$. La courbe thermogravimétrique à $150^{\circ} \mathrm{C} / \mathrm{min}$ coupe les deux autres courbes à $100^{\circ} \mathrm{C} / \mathrm{min}$ et $50^{\circ} \mathrm{C} / \mathrm{min}$ aux alentours de $900^{\circ} \mathrm{C}$ (figure 5.4 ).

En milieu oxydant, la courbe thermogravimétrique à $50^{\circ} \mathrm{C} / \mathrm{min}$ est la courbe caractéristique présentant une avance à basse température $\left(\mathrm{T}<900^{\circ} \mathrm{C}\right)$ et une perte de poids supérieure à haute température $\left(\mathrm{T}>900^{\circ} \mathrm{C}\right.$ ). La courbe thermogravimétrique à $50^{\circ} \mathrm{C} / \mathrm{min}$ englobe les deux autres courbes à $150^{\circ} \mathrm{C} / \mathrm{min}$ et $100^{\circ} \mathrm{C} / \mathrm{min}$; alors que ces deux dernières se coupent aux alentours de $1000^{\circ} \mathrm{C}$ (figure 6.5).

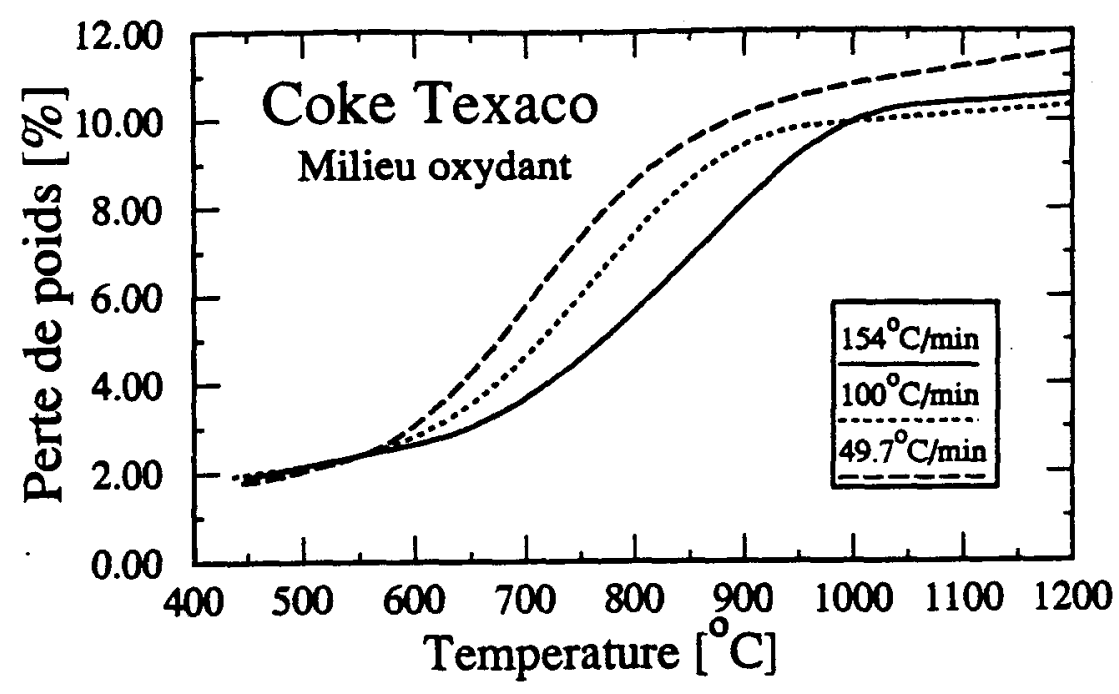

Figure 6.5: Effet du taux de chauffage sur le thermogramme du coke TEXACO, en milieu oxydant ( $95 \%$ azote, $5 \%$ oxygène). 


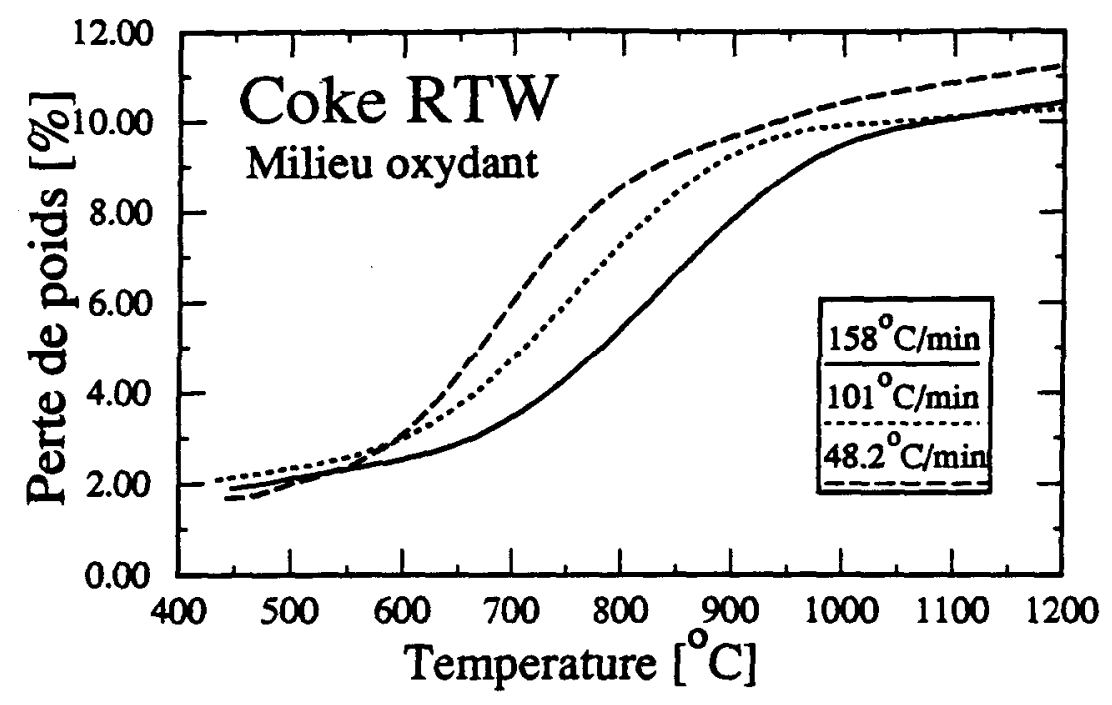

Figure 6.6: Effet du taux de chauffage sur le thermogramme du coke RTW, en milieu oxydant ( $95 \%$ azote, $5 \%$ oxygène).

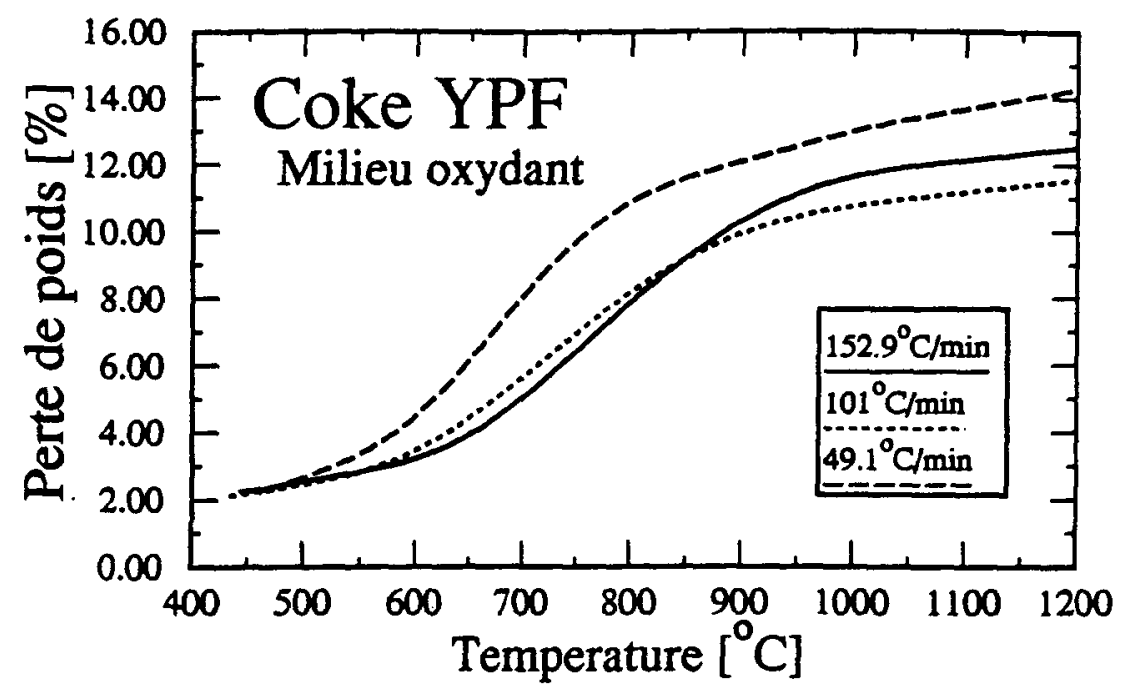

Figure 6.7 : Effet du taux de chauffage sur le thermogramme du coke YPF, en milieu oxydant ( $95 \%$ azote, $5 \%$ oxygène). 


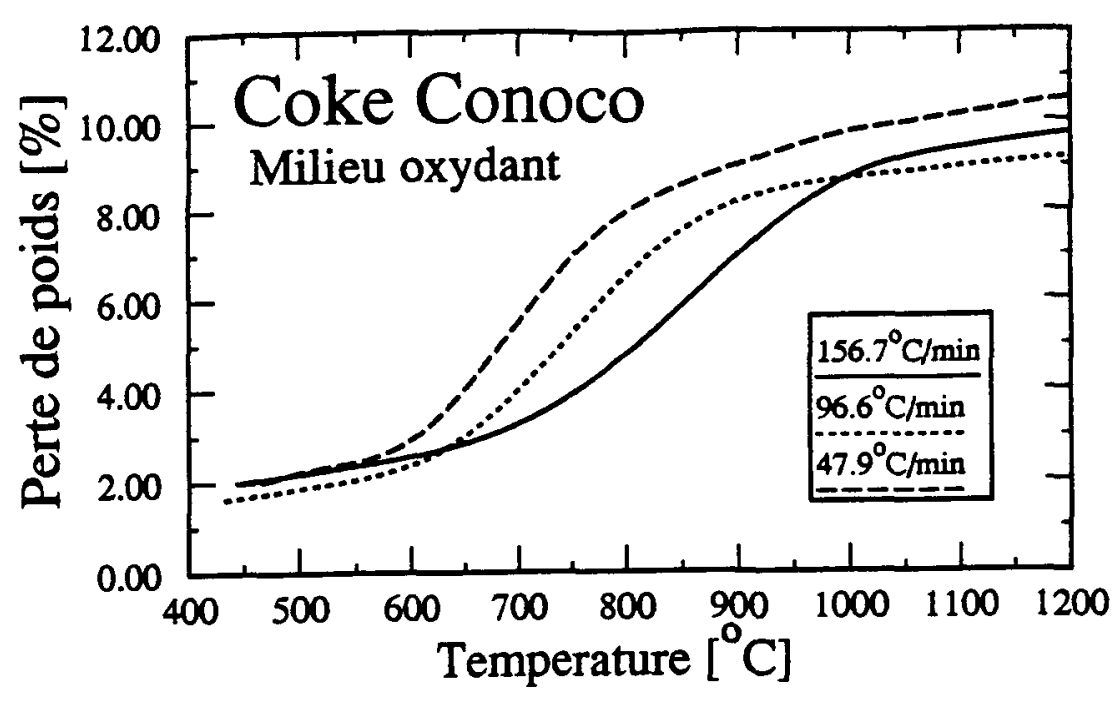

Figure 6.8 : Effet du taux de chauffage sur le thermogramme du coke CONOCO, en milieu oxydant ( $95 \%$ azote, $5 \%$ oxygène).

\section{VI.2.3 Comparaison des thermogrammes obtenus en milieux neutre et oxydant}

Les figures 6.9 à 6.11 montrent l'influence du milieu sur la courbe thermogravimettrique pour le coke TEXACO. On constate que le milieu oxydant retarde la dévolatilisation à basse température $\left(\mathrm{T}<900^{\circ} \mathrm{C}\right)$, surtout lorsque le taux de chauffage est élevé $\left(150^{\circ} \mathrm{C} / \mathrm{min}\right)$. Par contre à $50^{\circ} \mathrm{C} / \mathrm{min}$, la perte de poids suit la même évolution, les deux courbes thermogravimétriques se superposent jusqu'à $750^{\circ} \mathrm{C}$. A haute température $\left(\mathrm{T}>900^{\circ} \mathrm{C}\right.$ ), la différence entre les thermogrammes en milieux neutre et oxydant augmente au fur et à mesure que le taux de chauffage diminue; elle devient significative et croît au cours du chauffage à partir de $50^{\circ} \mathrm{C} / \mathrm{min}$. En effet, lorsque le taux de chauffage est suffisamment faible, la combustion du coke à haute tempêrature engendre une perte de poids cumulative croissante.

A $150^{\circ} \mathrm{C} / \mathrm{min}$, la vitesse de chauffage étant trop élevée, le coke n'entre pas en combustion à haute température. L'oxygène du milieu participe exclusivement à la combustion rapide des produits volatils dégagés de la masse de coke (figure 6.9). Cette affirmation est d'ailleurs vérifiée au regard 
de la figure 6.12, représentant la concentration des gaz restant lors de la pyrolyse du coke TEXACO en milieu oxydant. On constate qu'il n'y a plus de volatils présents dans le milieu à partir de $1100^{\circ} \mathrm{C}$ et que c'est précisément à partir de ce moment là que l'oxygène du milieu peut attaquer le résidu solide de coke, si toutefois une vitesse de chauffage suffisamment faible le permet; ce qui n'est pas le cas à $150^{\circ} \mathrm{C} / \mathrm{min}$ et probablement à $100^{\circ} \mathrm{C} / \mathrm{min}$.

A $50^{\circ} \mathrm{C} / \mathrm{min}$ par contre, les deux courbes thermogravimétriques en milieux neutre et oxydant suivent pratiquement la même évolution jusqu'à $750^{\circ} \mathrm{C}$. A partir de cette température, le thermogramme du milieu oxydant diverge par rapport au milieu neutre; l'oxygène présent dans le milieu réagit avec le coke (figure 6.11). En effet, la figure 6.12 montre qu'il n'y a plus de volatils présents dans le milieu à partir de $800^{\circ} \mathrm{C}$, lors de la pyrolyse du coke TEXACO à $50^{\circ} \mathrm{C} / \mathrm{min}$; ceci facilitant l'attaque du solide carboné par l'oxygène.

Au regard des figures présentées en annexe IV, les mêmes constatations peuvent être faites quant à la combustion du coke à partir de $50^{\circ} \mathrm{C} / \mathrm{min}$ pour les cokes RTW et YPF.

Par contre, le coke CONOCO, présentant le plus faible indice en matières volatiles, est attaqué par l'oxygène du milieu à partir de $100^{\circ} \mathrm{C} / \mathrm{min}$. Cette observation relève du fait que les volatils, en plus faible proportion dans le coke CONOCO, ne protègent plus aussi efficacement le résidu de coke de l'attaque par l'oxygène au cours de sa calcination, comme le montre la figure 6.13 présentant les produits volatils restant en milieu oxydant pour le coke CONOCO. 


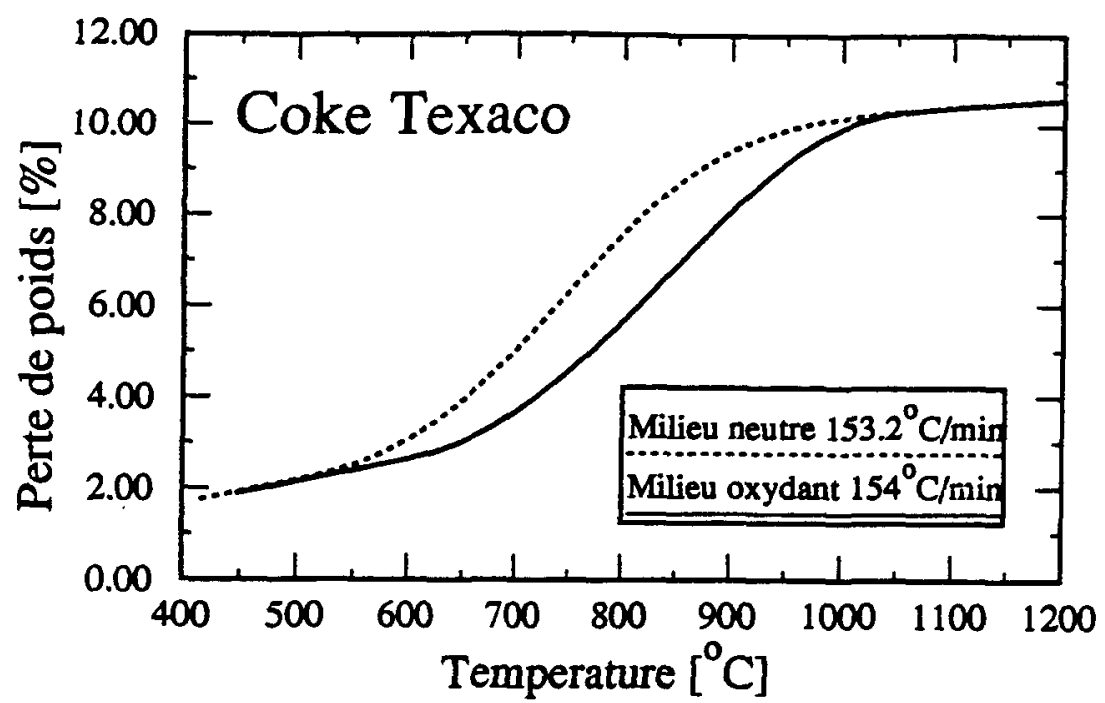

Figure 6.9: Comparaison des thermogrammes en milieux neutre et oxydant, à $150^{\circ} \mathrm{C} / \mathrm{min}$, pour le coke TEXACO.

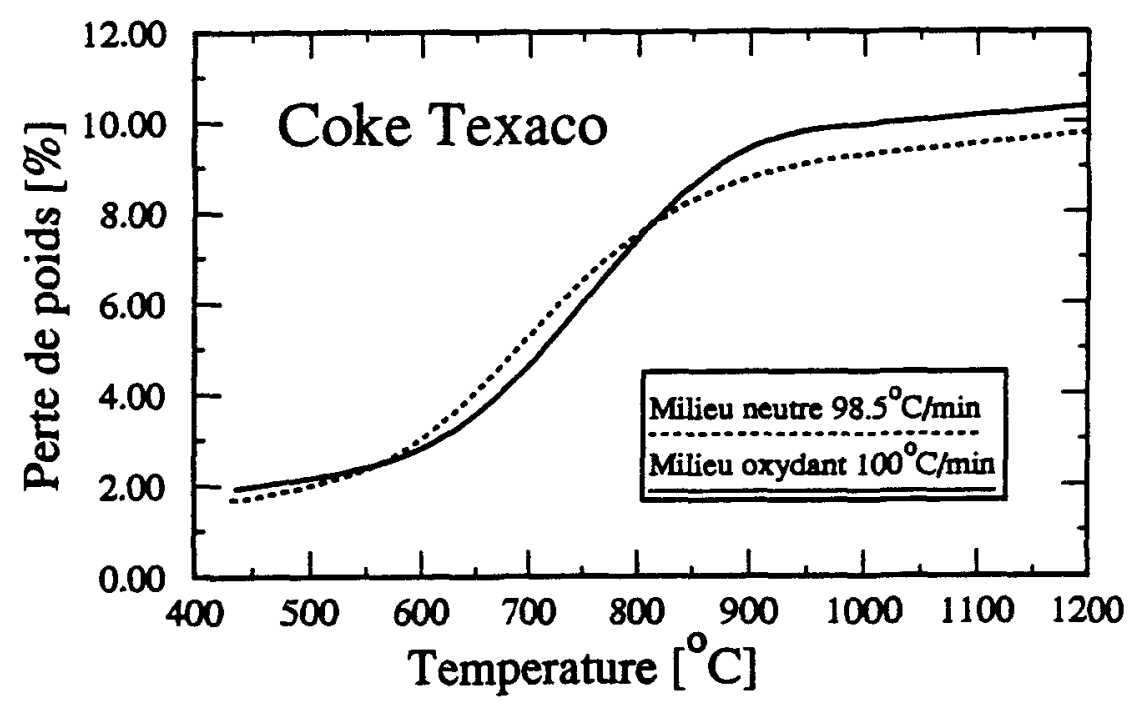

Figure 6.10 : Comparaison des thermogrammes en milieux neutre et oxydant, à $100^{\circ} \mathrm{C} / \mathrm{min}$, pour le coke TEXACO. 


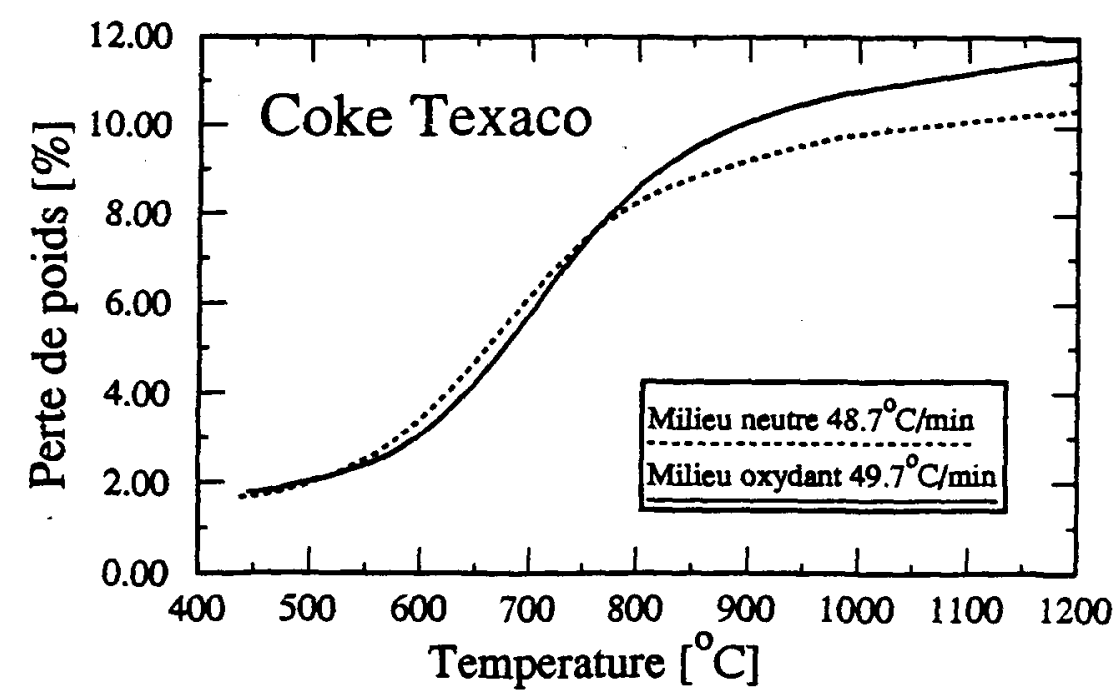

Figure 6.11: Comparaison des thermogrammes en milieux neutre et oxydant, à $50^{\circ} \mathrm{C} / \mathrm{min}$, pour le coke TEXACO.

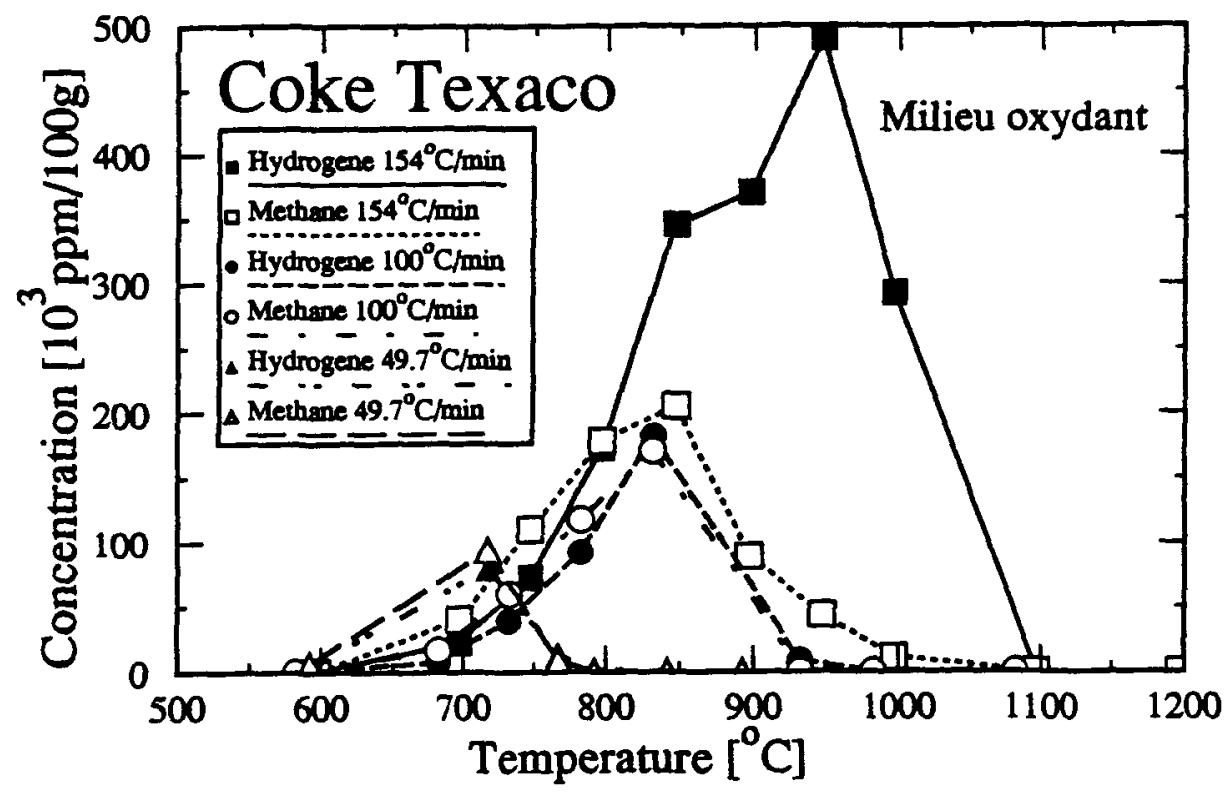

Figure 6.12: Effet du taux de chauffage sur l'hydrogène et le méthane en milieu oxydant (95\% azote, $5 \%$ oxygène). 


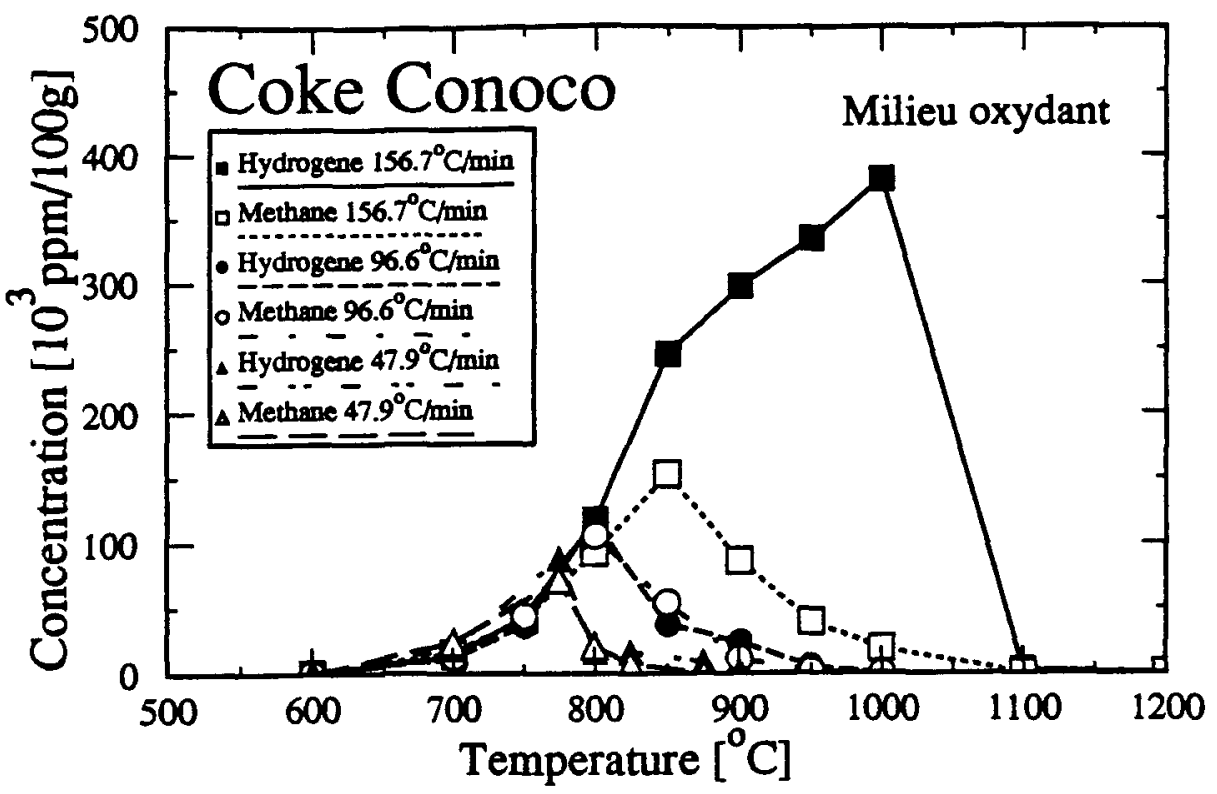

Floure 6.13: Effet du taux de chauffage sur l'hydrogène et le méthane pour le coke CONOCO

\section{VI.3 Analyse des gaz de dévolatilisation: Hydrogène et méthane}

En présence d'air, les produits volatils (condensables, méthane et hydrogène) une fois dégagés de la masse solide de coke, entrent en combustion avec l'oxygène présent dans le milieu. Cette combustion est rapide, et se déroule en phase gazeuse. En milieu neutre, la présence des vapeurs de goudron à partir de $550^{\circ} \mathrm{C}$ se caractérisait par une fumée brune, dense, typique des goudrons. En atmosphère oxydante par contre, les goudrons réagissent avec l'oxygène pour former des produits de combustion contenant entre autre une quantité appréciable d'eau. On peut d'ailleurs constater visuellement la présence d'une fumée blanche, chargée de vapeur d'eau entre $550^{\circ} \mathrm{C}$ et $700^{\circ} \mathrm{C}$, au lieu de la fumée typiquement brune du milieu neutre.

\section{VI.3.1 Comparaison des cokes}

Les figures 6.14 à 6.16 représentent la concentration des gaz restant (hydrogène et méthane) pour les quatre types de coke, et ce pour les trois taux de chauffage. Le coke YPF présente le pic le plus important, il 
correspond au coke contenant le plus haut pourcentage en matières volatiles. Le coke CONOCO, plus faible en matières volatiles, présente le pic le moins important. Les cokes TEXACO et RTW, très proches en ce qui concernent leurs propriétés intrinsèques, présentent des pics voisins en amplitude.

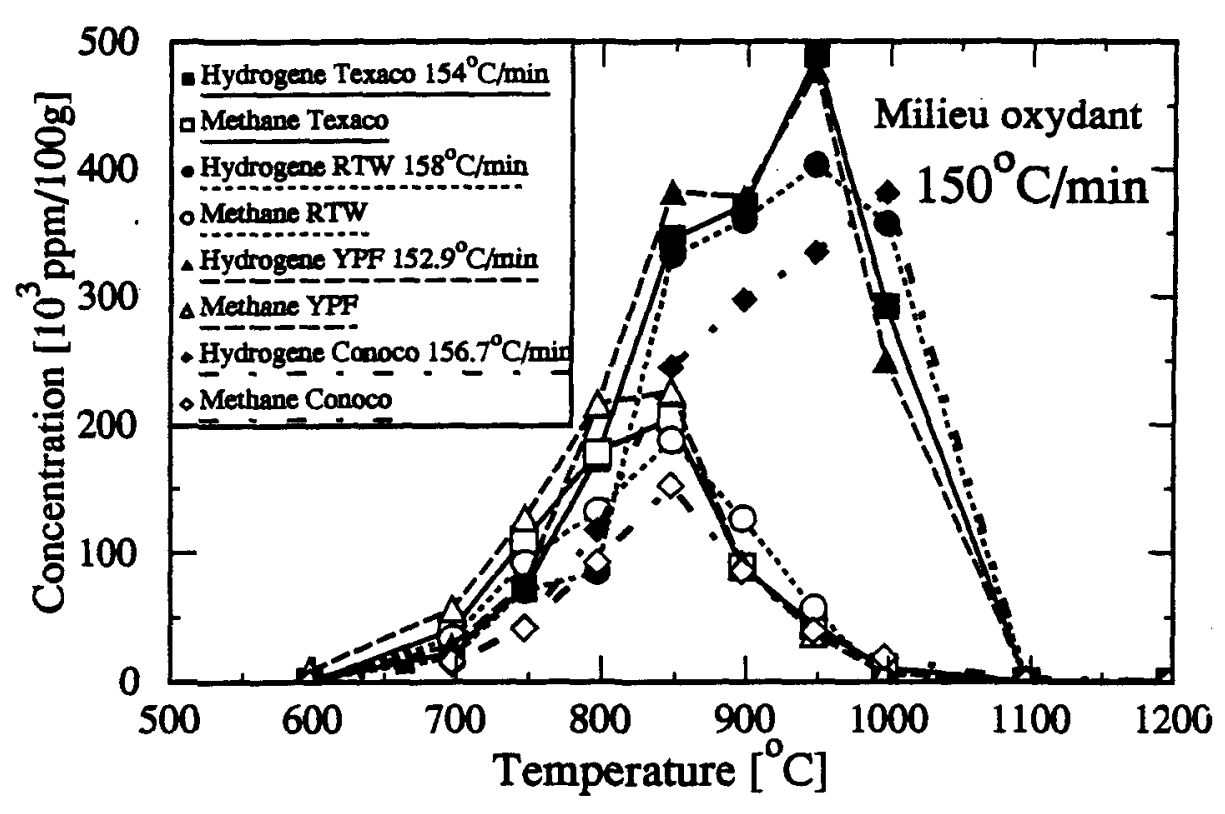

Flgure 6.14: Comparaison de l'hydrogène et du méthane à $150^{\circ} \mathrm{C} / \mathrm{min}$, en milleu oxydant ( $95 \%$ azote, $5 \%$ oxygène). 


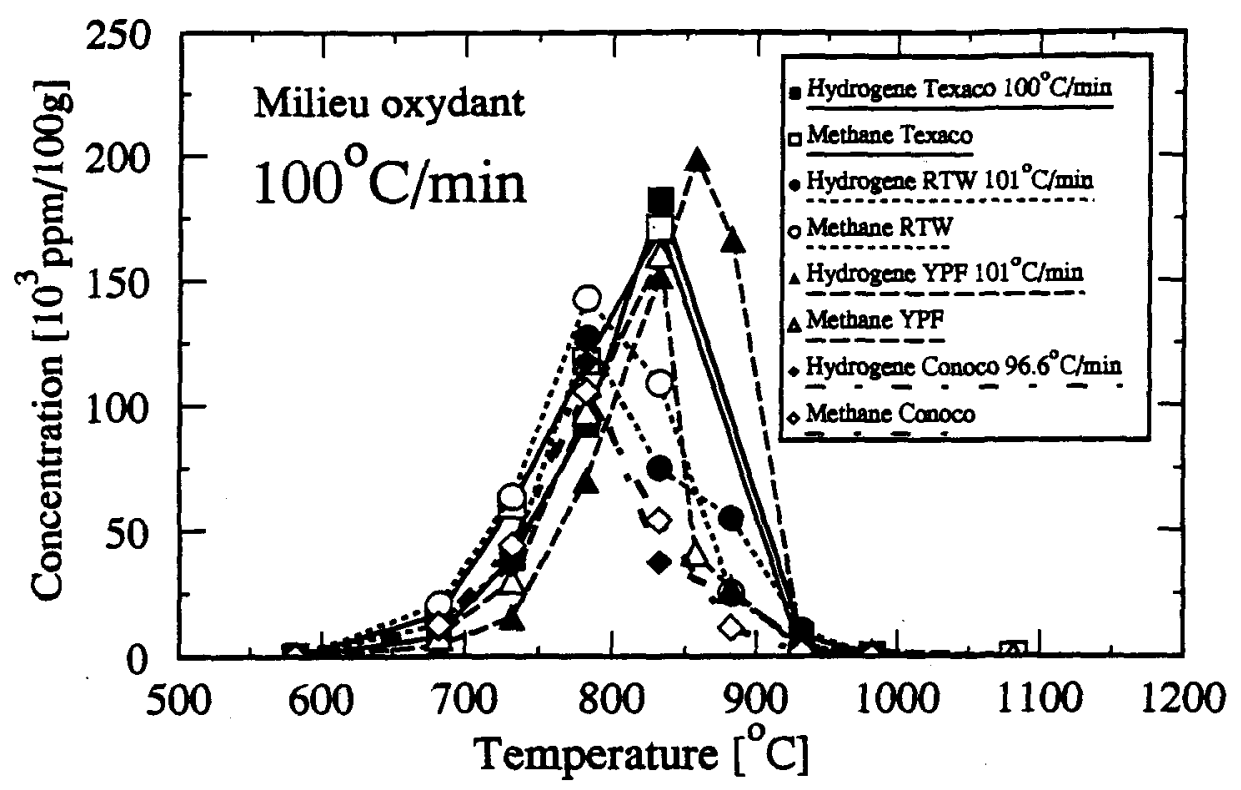

Elgure 6.15: Comparaison de l'hydrogène et du méthane à $100^{\circ} \mathrm{C} / \mathrm{min}$, en milleu oxydant ( $95 \%$ azote, $5 \%$ oxygène).

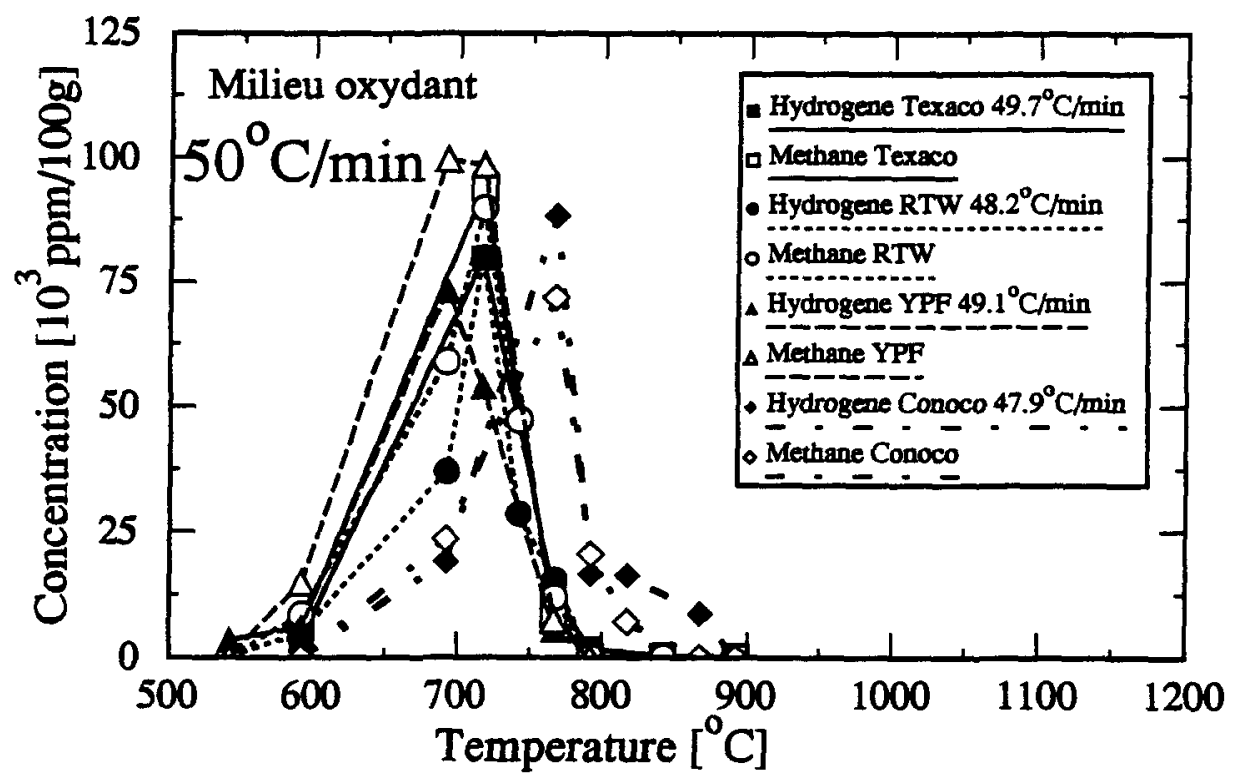

Figure 6.16: Comparaison de l'hydrogène et du méthane à $50^{\circ} \mathrm{C} / \mathrm{min}$, en milleu oxydant ( $95 \%$ azote, $5 \%$ oxygène). 


\section{V.3.2 Effet du taux de chauffage}

Les figures 6.17 et 6.18 révèlent l'effet du taux de chauffage sur les concentrations en hydrogène et mẻthane restant pour le coke TEXACO.

A $150^{\circ} \mathrm{C} / \mathrm{min}$, il ne reste pratiquement plus d'hydrogène à $1100^{\circ} \mathrm{C}$; au fur et à mesure que le taux de chauffage diminue, cette limite se déplace vers les basses températures: à $100^{\circ} \mathrm{C} / \mathrm{min}$ la limite se situe à $950^{\circ} \mathrm{C}$; à $50^{\circ} \mathrm{C} / \mathrm{min}$ elle se situe à $800^{\circ} \mathrm{C}$.

A l'examen des figures 6.14 à 6.16 en milieu oxydant, on constate qu'à $150^{\circ} \mathrm{C} / \mathrm{min}$, le pic du méthane se situe environ $100^{\circ} \mathrm{C}$ avant le pic de l'hydrogène. Ce même écart de $100^{\circ} \mathrm{C}$ a d'ailleurs été constaté en milieu neutre, pour les trois taux de chauffage (voir figures 5.8 à 5.10).

Par contre, lorsque le taux de chauffage diminue, les pics d'hydrogène et de méthane se superposent entre 775 et $850^{\circ} \mathrm{C}$ pour le taux de chauffage de $100^{\circ} \mathrm{C} / \mathrm{min}$ et entre 700 et $775^{\circ} \mathrm{C}$ pour $50^{\circ} \mathrm{C} / \mathrm{min}$. Au cours de la pyrolyse du coke vert en atmosphère oxydante, plus le taux de chauffage est faible $\left(100\right.$ et $\left.50^{\circ} \mathrm{C} / \mathrm{min}\right)$, plus la combustion des produits volatils de dégazéification est favorisée, à cause de la durée de l'expérience qui tient les volatils plus longtemps en présence de l'oxygène comburant. Les mêmes observations sont faites pour les trois autres types de coke.

L'amplitude du pic de concentration des gaz diminue lorsque le taux de chauffage diminue mais il ne s'agit là que d'une valeur instantanée, logiquement plus élevée lorsque le taux de chauffage est élevé. Cela ne signifie pas que le volume total d'hydrogène ou de méthane présent à $150^{\circ} \mathrm{C} / \mathrm{min}$ est plus élevé, car il faut rapporter ces concentrations instantanées sur l'échelle du temps. 


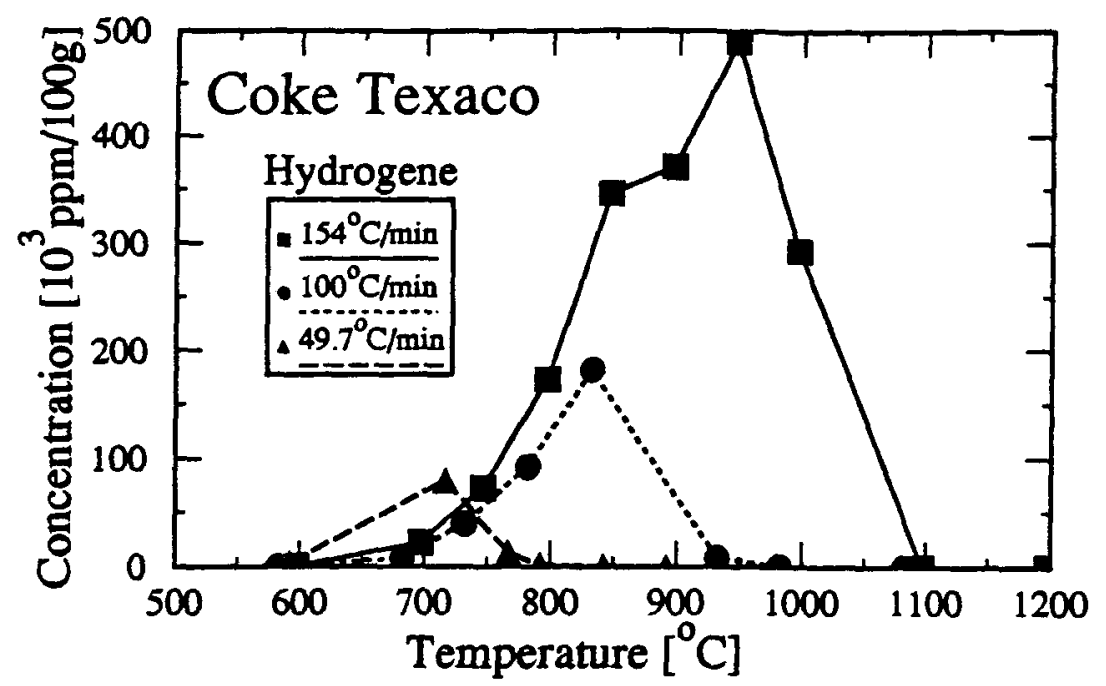

FIgure 6.17: Effet du taux de chauffage sur l'hydrogène du coke TEXACO, en milleu oxydant ( $95 \%$ azote, $5 \%$ oxygène).

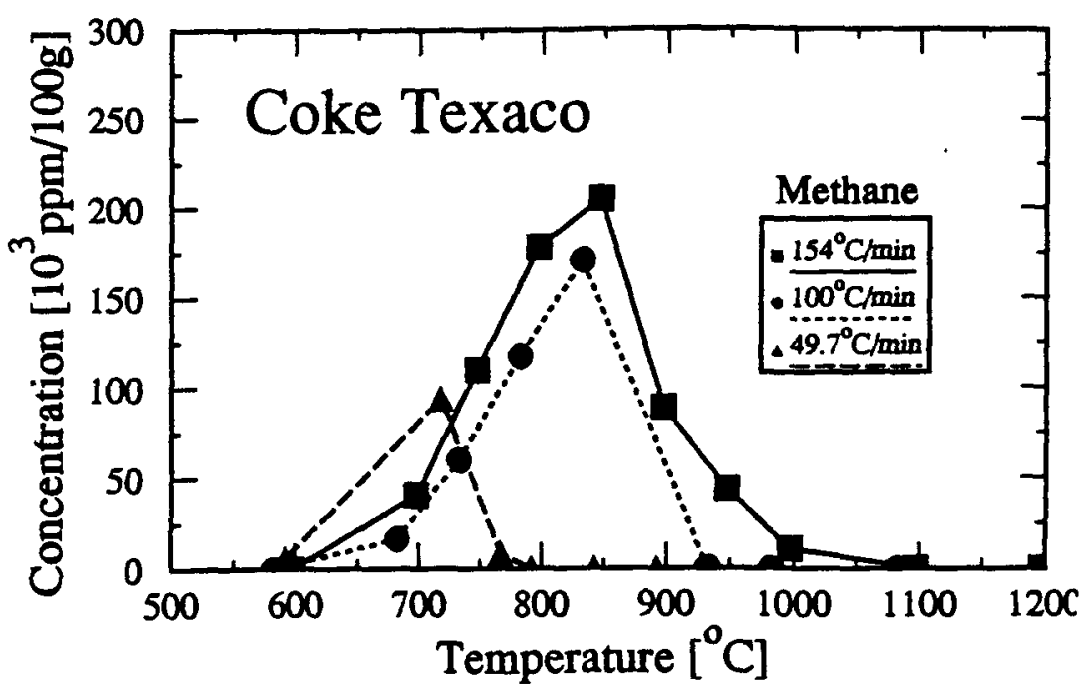

Elgure 6.18: Effet du taux de chauffage sur le méthane du coke TEXACO, en milleu oxydant (95\% azote, $5 \%$ oxygène). 
VI.4 Analyse des gaz: Monoxyde et dioxyde de carbone

Le monoxyde de carbone provient principalement de la réaction entre le carbone du coke et l'oxygène atomique qui lui est intimement lié. Cette affirmation est confirmée par des spectres de concentration en CO très proches, en milieux neutre et oxydant. Le dioxyde de carbone par contre, totalement absent en atmosphère neutre, se retrouve en quantités plus ou moins importantes en milieu oxydant, suite aux réactions de combustion.

\section{VI.4.1 Comparaison des cokes}

Les figures 6.19 à 6.21 représentent les concentrations en monoxyde et dioxyde de carbone au cours de la pyrolyse des quatre types de coke. Le coke YPF contenant le plus haut pourcentage de volatils se distingue par la concentration la plus élevée.

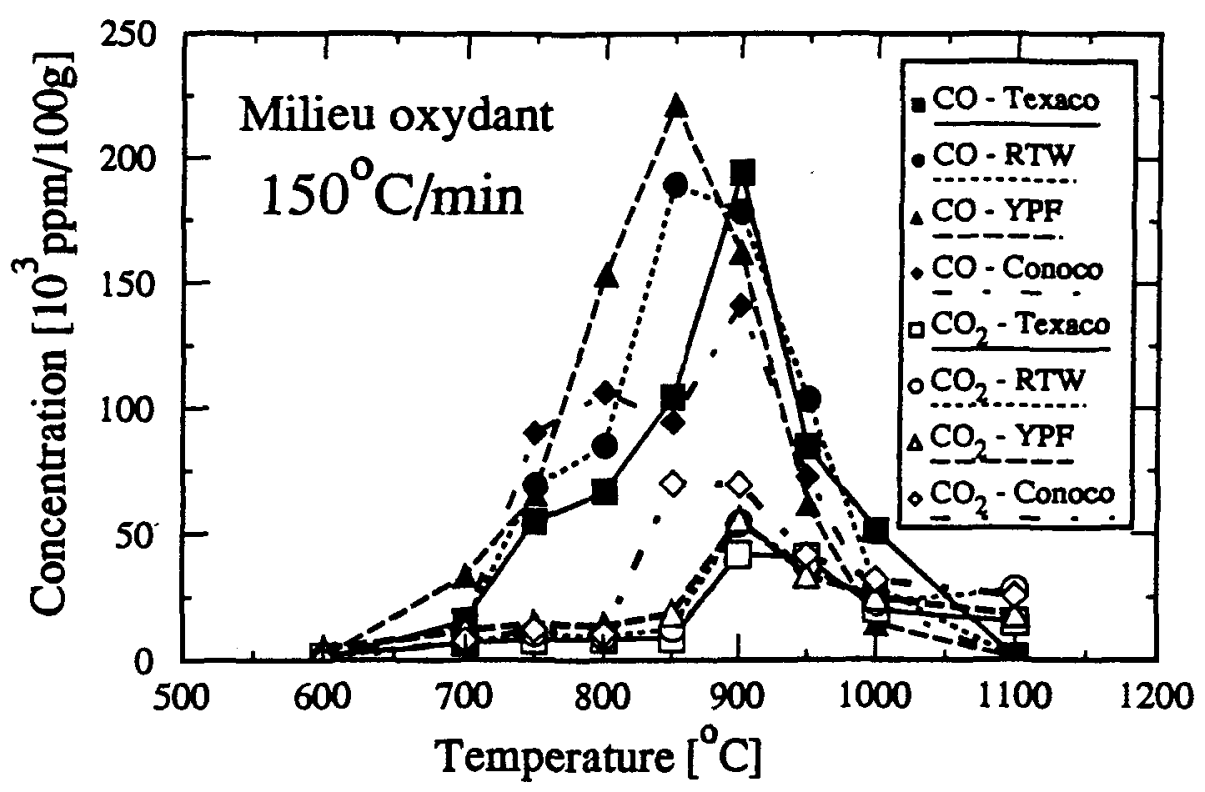

Figure 6.19: Comparaison des monoxyde et dioxyde de carbone à $150^{\circ} \mathrm{C} / \mathrm{min}$, en milleu oxydant ( $95 \%$ azote, $5 \%$ oxygène). 


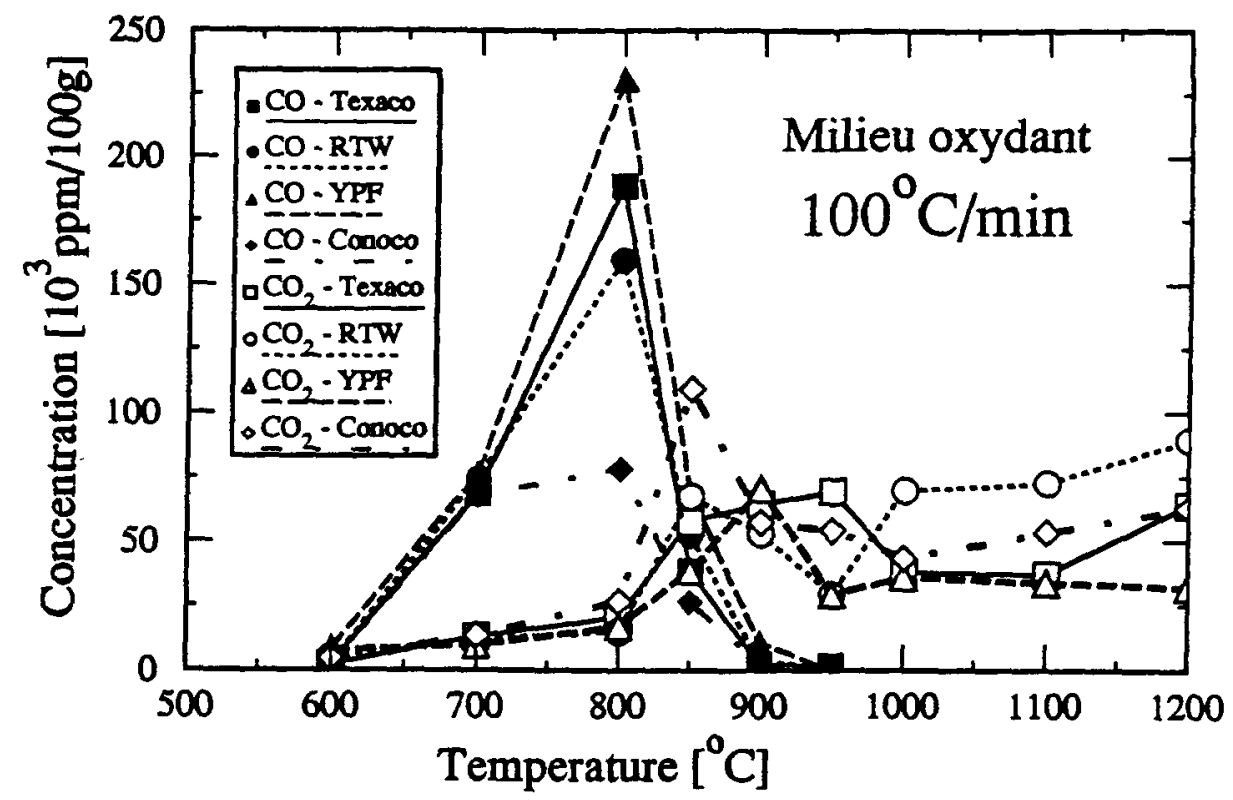

Figure 6.20 : Comparaison des monoxyde et dioxyde de carbone à $100^{\circ} \mathrm{C} / \mathrm{min}$, en milleu oxydant ( $95 \%$ azote, $5 \%$ oxygène).

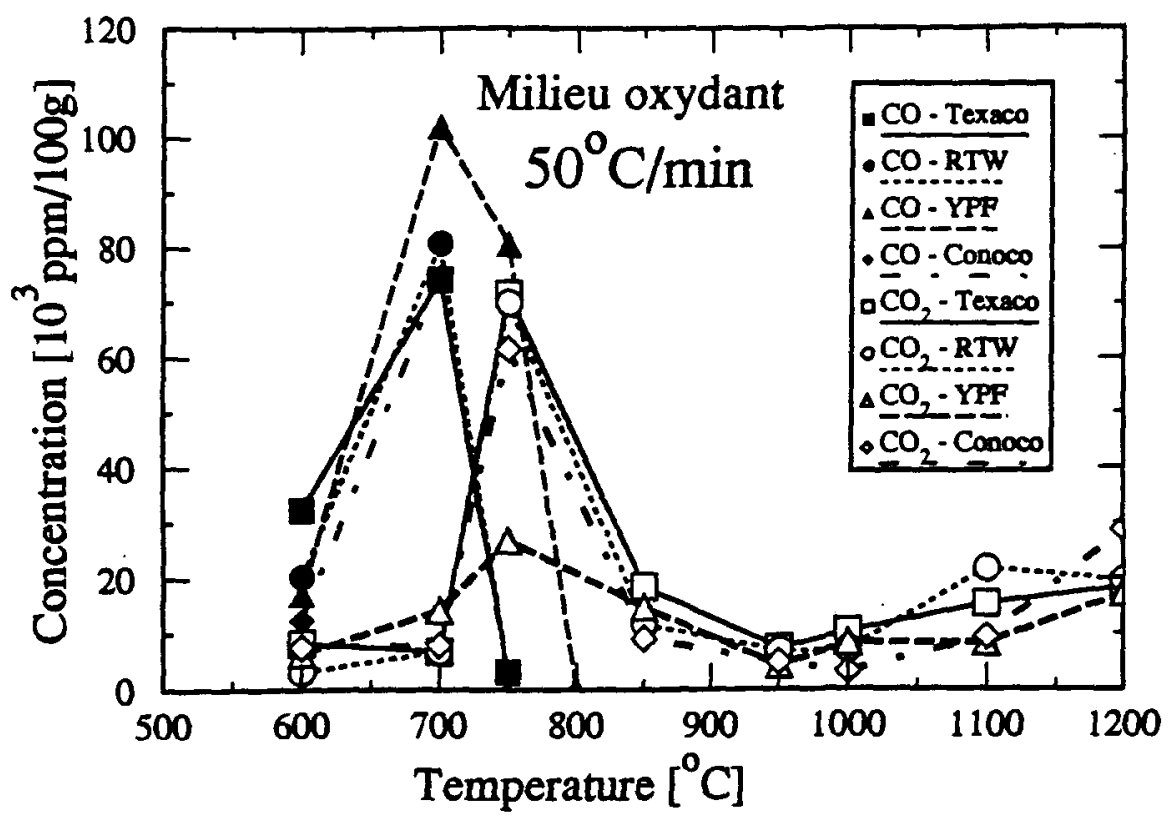

Figure 6.21: Comparaison des monoxyde et dioxyde de carbone à $50^{\circ} \mathrm{C} / \mathrm{min}$, en milleu oxydant $(95 \%$ azote, $5 \%$ oxygène). 


\section{VI.4.2 Effet du taux de chauffage}

Les figures 6.22 et 6.23 révèlent l'effet du taux de chauffage sur les monoxyde et dioxyde de carbone prélevés au cours de la pyrolyse du coke TEXACO. On constate sur ces figures que le $\mathrm{CO}_{2}$ se forme dans le deuxième stade de la calcination, après la formation de $\mathrm{CO}$, et ce quel que soit le taux de chauffage. Le rapport $\mathrm{CO}_{2} / \mathrm{CO}$ augmente rapidement avec la diminution du taux de chauffage à cause des réactions de combustion favorisées lors du chauffage lent.

Si l'on compare la figure 5.15 présentant le CO recueilli en milieu neutre, avec la figure 6.22 relative au milieu oxydant, on constate que l'émission du CO ne semble pas être influencée par la nature du milieu, tout au moins à haute vitesse de chauffage $\left(100\right.$ et $\left.150^{\circ} \mathrm{C} / \mathrm{min}\right)$. A $50^{\circ} \mathrm{C} / \mathrm{min}$ par contre, la quantité de $\mathrm{CO}$ en milieu oxydant est plus faible par rapport au milieu neutre, et ce à cause de la combustion secondaire du $\mathrm{CO}$ en $\mathrm{CO}_{2}$. Ce fait est d'ailleurs confirmé par l'existence d'un pic important de $\mathrm{CO}_{2}$. directement successif au pic de $\mathrm{CO}$ à $50^{\circ} \mathrm{C} / \mathrm{min}$ (figure 6.23). Les mêmes constatations sont faites pour les trois autres types de coke.

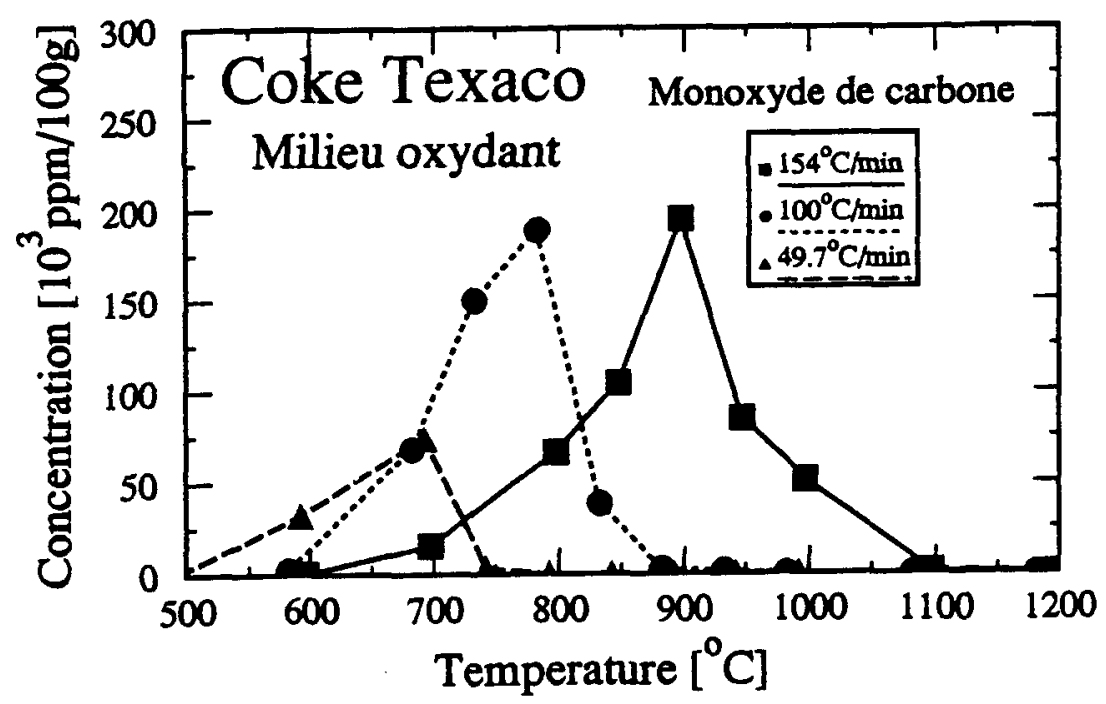

Figure 6.22 : Effet du taux de chauffage sur le monoxyde de carbone du coke TEXACO, en milieu oxydant ( $95 \%$ azote, $5 \%$ oxygène). 


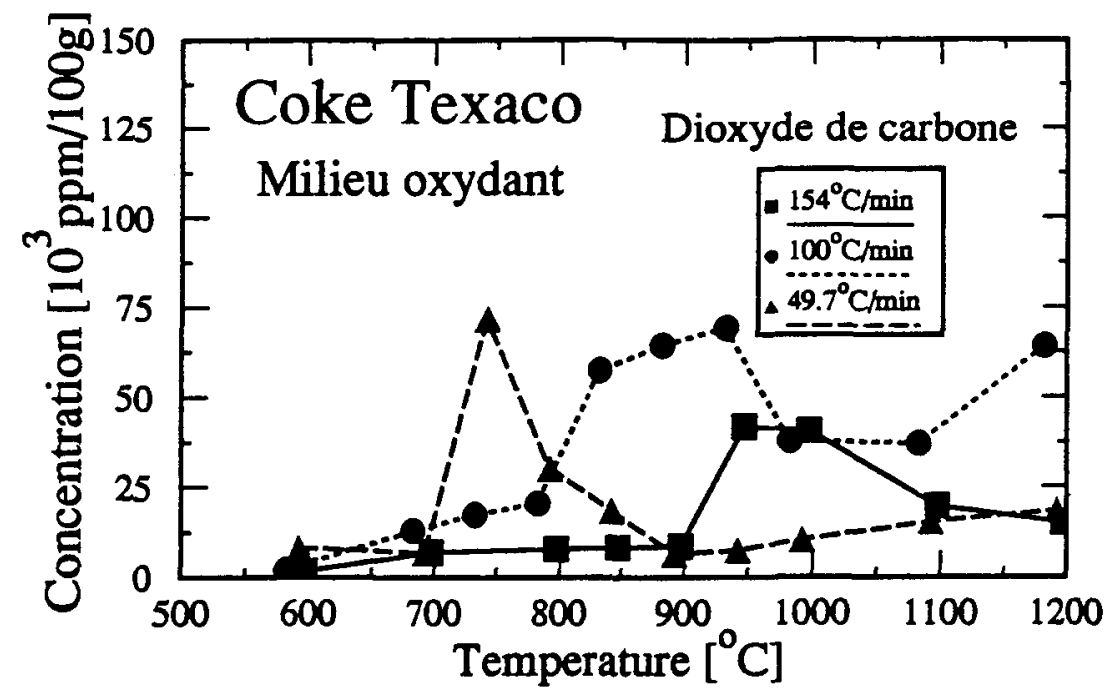

Figure 6.23 : Effet du taux de chauffage sur le dioxyde de carbone du coke TEXACO, en milleu oxydant ( $95 \%$ azote, $5 \%$ oxygène).

\section{VI.5 Comparaison des vitesses instantanées en milieux neutre et oxydant}

\section{VI.5.1 En ce qui concerne les vitesses instantanées totales}

Les figure 6.24 à 6.26 comparent les dérivées des courbes thermogravimétriques obtenues en milieux neutre et oxydant, pour le coke TEXACO aux trois taux de chauffage. On constate que la pente caractéristique de la courbe thermogravimétrique entre 600 et $1000^{\circ} \mathrm{C}$, représentée par l'amplitude de la dérivée, est plus élevée en milieu oxydant. Cette constatation peut être faite pour les trois autres types de coke également (annexe V).

D'autre part, on remarque de nouveau le décalage dans le temps des dérivées totales, comme précédemment cité. Ce décalage est plus marqué à haut taux de chauffage; plus ce dernier diminue plus le décalage s'amoindrit pour finalement être inexistant à $50^{\circ} \mathrm{C} / \mathrm{min}$. En effet, à $50^{\circ} \mathrm{C} / \mathrm{min}$, les deux dérivées en milieux neutre et oxydant se superposent parfaitement, avec la 
seule différence d'amplitude marquant une pente plus accentuée pour le milieu oxydant, à cause de la combustion du résidu de coke à partir de $750^{\circ} \mathrm{C}$ (figure 6.11 ). En annexe $\mathrm{V}$, on trouve les courbes instantanées pour les trois autres types de coke.

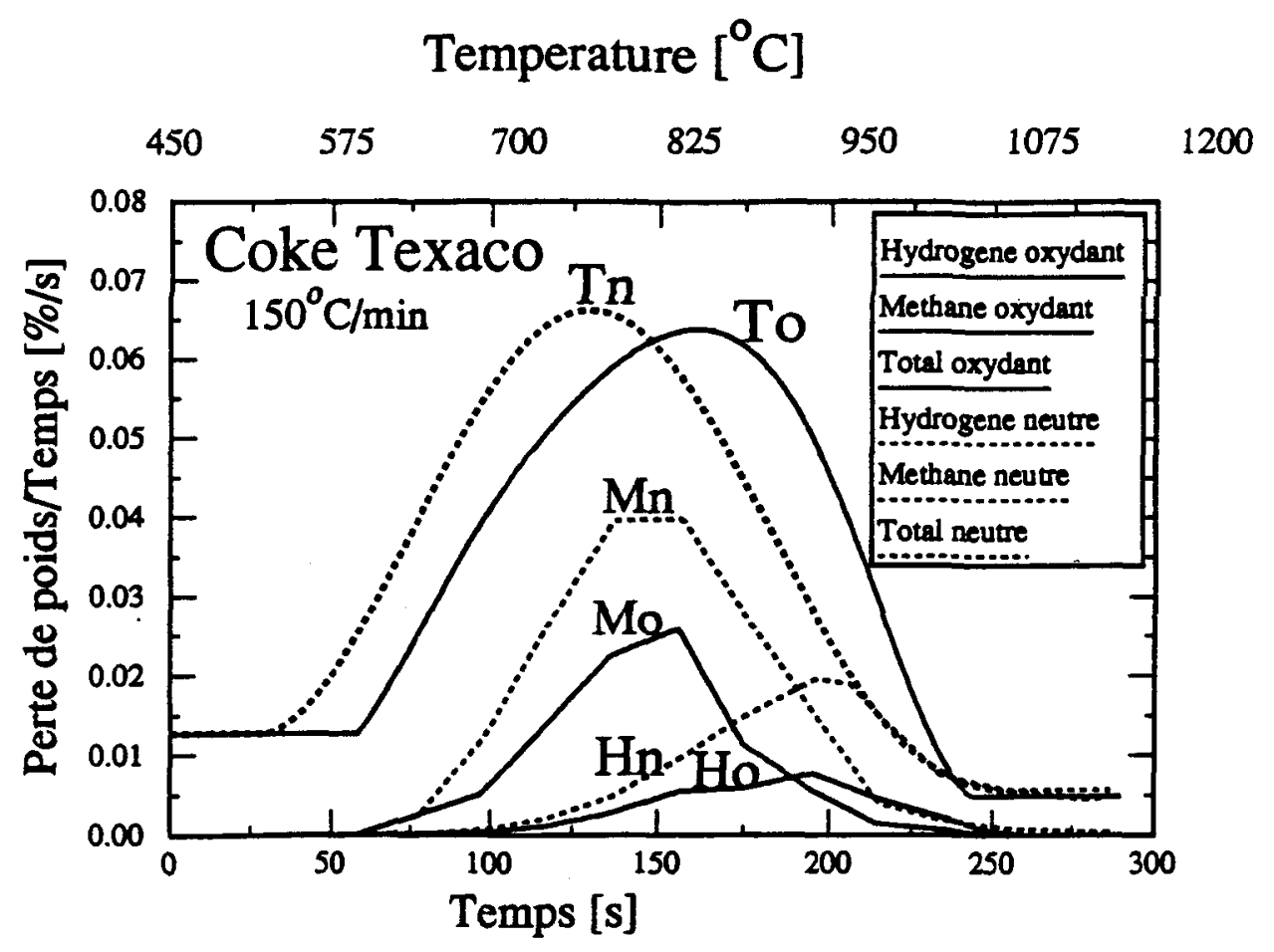

Figure 6.24 : Comparaison des valeurs instantanées en milieux neutre et oxydant, à $150^{\circ} \mathrm{C} / \mathrm{min}$, pour le coke TEXACO. 


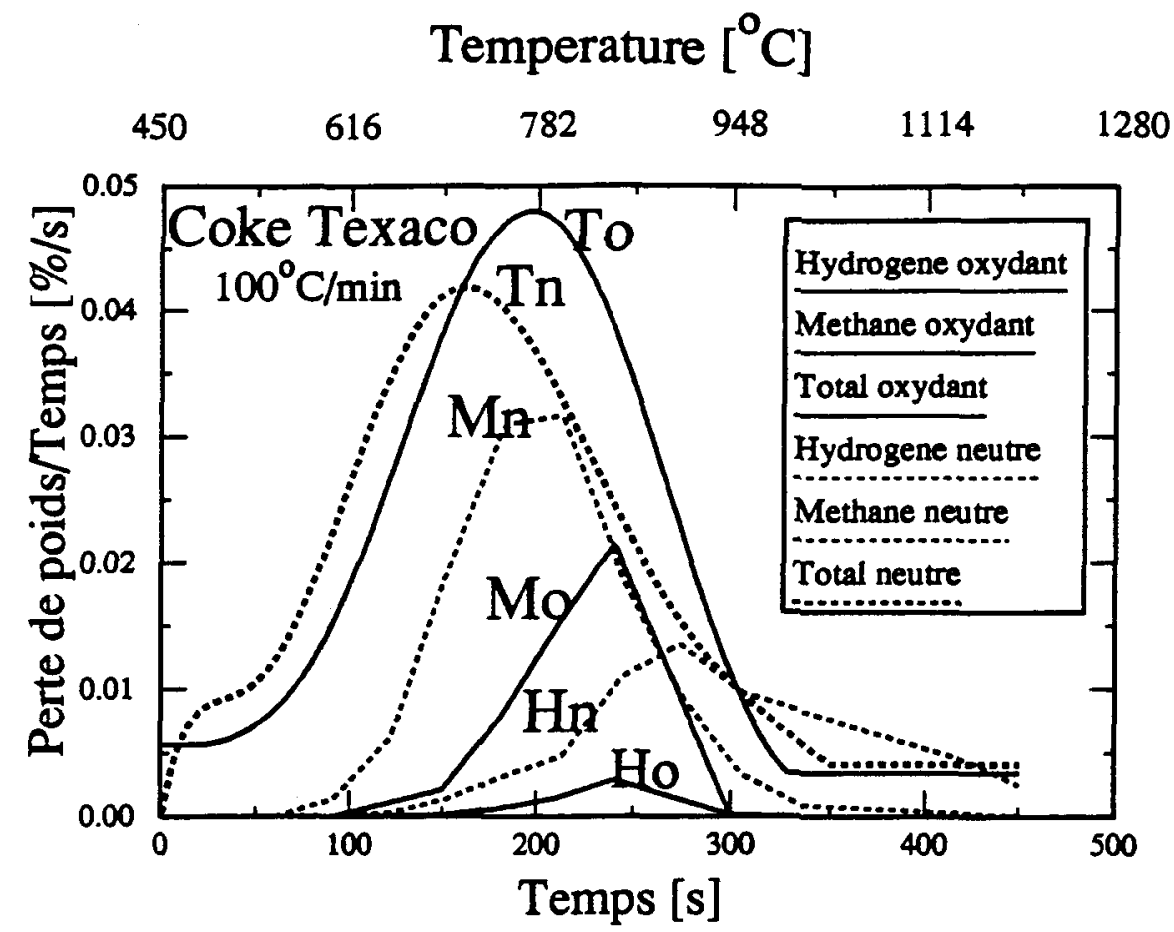

Figure 6.25 : Comparaison des valeurs instantanées en millieux neutre et oxydant, à $100^{\circ} \mathrm{C} / \mathrm{min}$, pour le coke TEXACO.

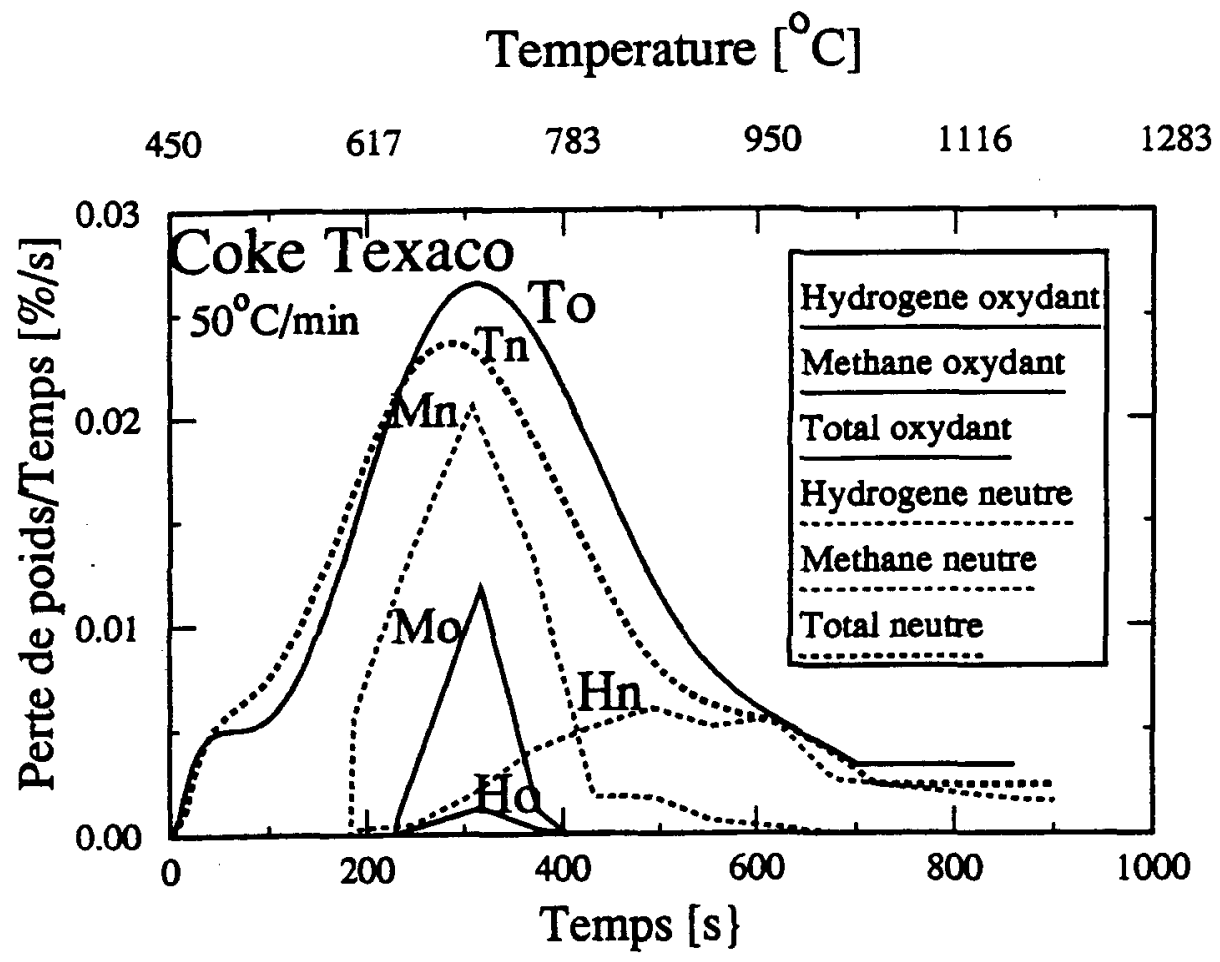

Flgure 6.26: Comparaison des valeurs instantanées en milleux neutre et oxydant, à $50^{\circ} \mathrm{C} / \mathrm{min}$, pour le coke TEXACO. 
V.5.2 En ce qui concerne les vitesses instantanées relatives aux produits: Hydrogène et méthane

La vitesse instantanée relative aux produits s'exprime en g/s et est rapportée à 100 grammes de coke vert. Elle est obtenue en multipliant la concentration en ppm par le débit total $(10.6 \mathrm{~L} / \mathrm{min})$ et le poids molaire du produit en $\mathrm{g} / \mathrm{mol}$, et en divisant par le volume molaire $(22.4 \mathrm{~L} / \mathrm{mol})$.

On constate que la courbe instantanée des produits restant du milieu oxydant est exactement englobée par la courbe instantanée des produits de dévolatilisation du milieu neutre, et ce aux trois taux de chauffage. La différence entre les deux courbes représente la combustion de ces mêmes produits volatils au cours de la calcination (figures 6.24 à 6.26).

\section{VI.5.3 En ce qui concerne les courbes cumulatives relatives aux produits: Hydrogène et méthane}

L'intégration des courbes instantanées donnent les courbes cumulatives relatives aux produits volatils. Les figures 6.27 à 6.29 comparent les courbes cumulatives relatives aux deux milieux, aux trois taux de chauffage respectivement.

En milieu oxydant, les courbes $H_{0}$ et $M_{0}$ représentent l'hydrogène et le méthane dans le milieu au cours de la calcination, c'est-à-dire n'ayant pas subi la combustion (phénomène de dévolatilisation-combustion).

En milieu neutre par contre, les courbes $H_{n}$ et $M_{n}$ représentent l'hydrogène et le méthane dégagés au cours de la calcination (phénomène de dévolatilisation uniquement).

En milieu oxydant à $150^{\circ} \mathrm{C} / \mathrm{min}$ (figure 6.27 ), on remarque le palier présent sur la courbe cumulative de l'hydrogène $\left(\mathrm{H}_{0}\right)$ à partir de 260 secondes $\left(1100^{\circ} \mathrm{C}\right)$, et du méthane $\left(\mathrm{M}_{0}\right)$ à partir de 250 secondes $\left(1075^{\circ} \mathrm{C}\right)$. Ceci signifie qu'après $1100^{\circ} \mathrm{C}$, les produits de la dévolatilisation du coke TEXACO ne se retrouvent plus dans le milieu parce qu'ils sont entièrement consommés par la combustion. En milieu neutre, ce palier existait uniquement pour le méthane dont l'émission était achevée à $1000^{\circ} \mathrm{C}$; par 
contre à $1200^{\circ} \mathrm{C}$, l'émission d'hydrogène n'était pas encore achevée (se référer aux figures 5.20 et 5.21 ).

Les figures 6.30 et 6.31 montrent l'effet du taux de chauffage sur les courbes cumulatives relatives aux produits $\mathrm{H}_{2}$ et $\mathrm{CH}_{4}$ au cours de la pyrolyse du coke TEXACO en atmosphère oxydante. On constate sur la figure 6.30 qu'à faible taux de chauffage $\left(50^{\circ} \mathrm{C} / \mathrm{min}\right)$, il reste de l'hydrogène dans un intervalle de températures très limité (entre 650 et $750^{\circ} \mathrm{C}$ ); le palier existant après $750^{\circ} \mathrm{C}$ signifie qu'il n'y a plus d'hydrogène présent dans le milieu. Au fur et à mesure que l'on augmente la vitesse de chauffage, cet intervalle s'élargit entre 650 et $900^{\circ} \mathrm{C}$ à $100^{\circ} \mathrm{C} / \mathrm{min}$, et entre 650 et $1000^{\circ} \mathrm{C}$ à $150^{\circ} \mathrm{C} / \mathrm{min}$.

Quant au méthane, à $50^{\circ} \mathrm{C} / \mathrm{min}$, on en retrouve entre 650 et $720^{\circ} \mathrm{C}$; cet intervalle s'élargit entre 650 et $900^{\circ} \mathrm{C}$ à $100^{\circ} \mathrm{C} / \mathrm{min}$, et entre 650 et $1000^{\circ} \mathrm{C}$ à $150^{\circ} \mathrm{C} / \mathrm{min}$ (figure 6.31 ).

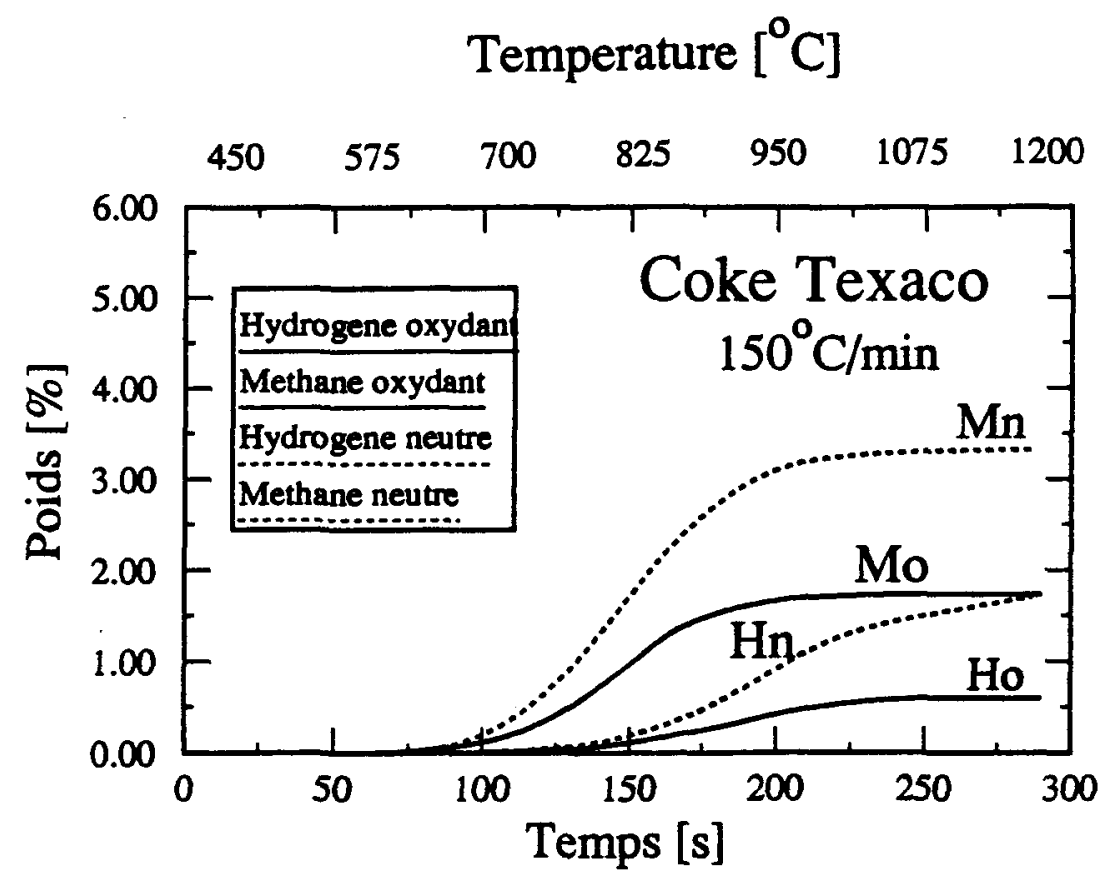

Figure 6.27 : Comparaison des valeurs cumulatives en milieux neutre et oxydant, à $150^{\circ} \mathrm{C} / \mathrm{min}$, pour le coke TEXACO. 
Temperature $\left[{ }^{\circ} \mathrm{C}\right]$

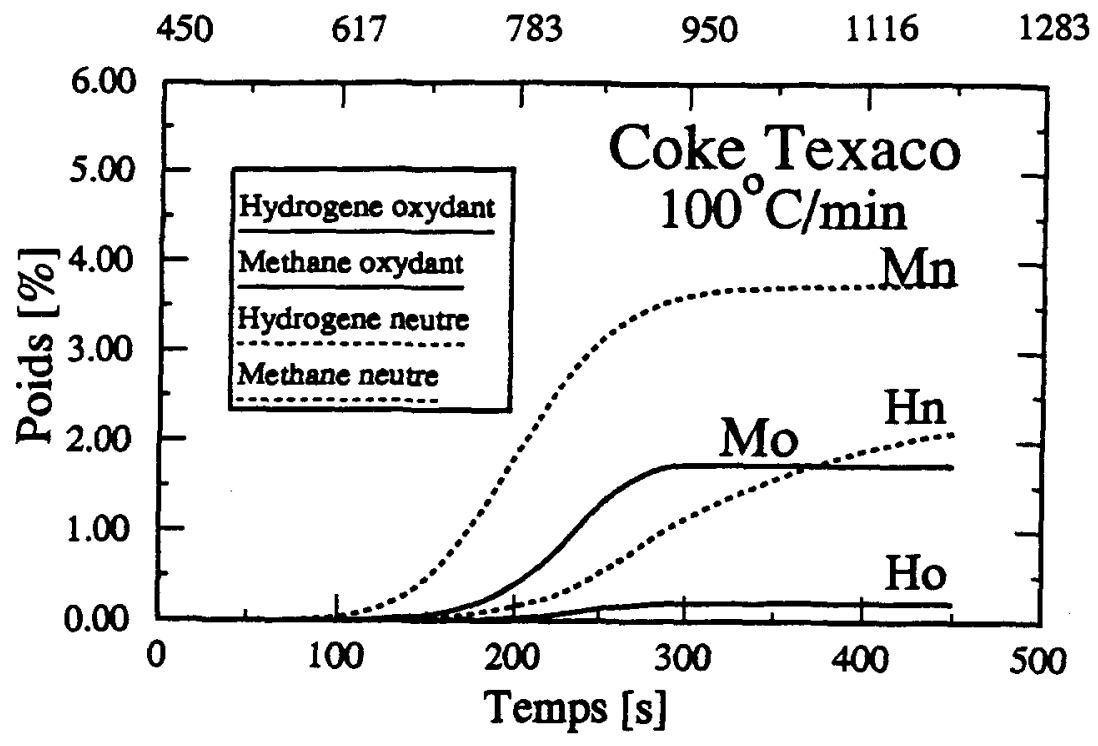

Figure 6.28 : Comparaison des valeurs cumulatives en milieux neutre et oxydant, à $100^{\circ} \mathrm{C} / \mathrm{min}$, pour le coke TEXACO.

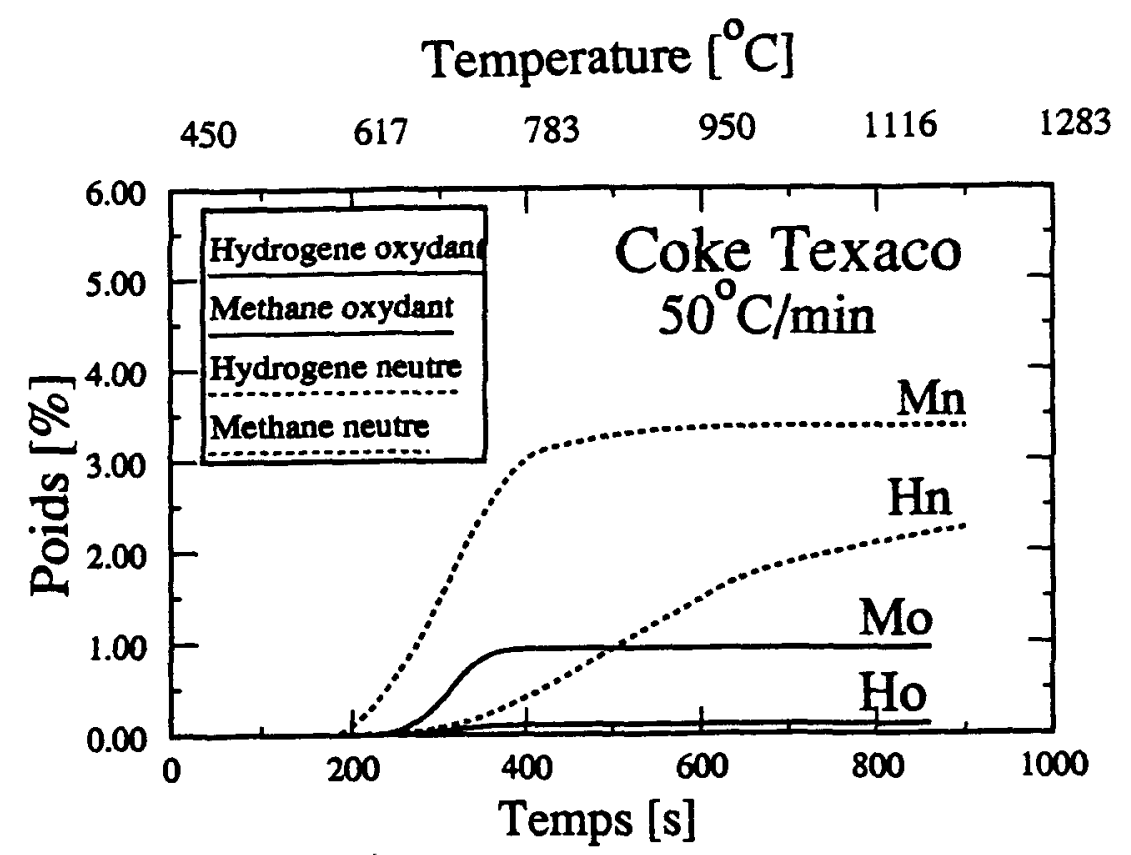

Flgure 6.29: Comparaison des valeurs cumulatives en milleux neutre et oxydant, à $50^{\circ} \mathrm{C} / \mathrm{min}$, pour le coke TEXACO. 


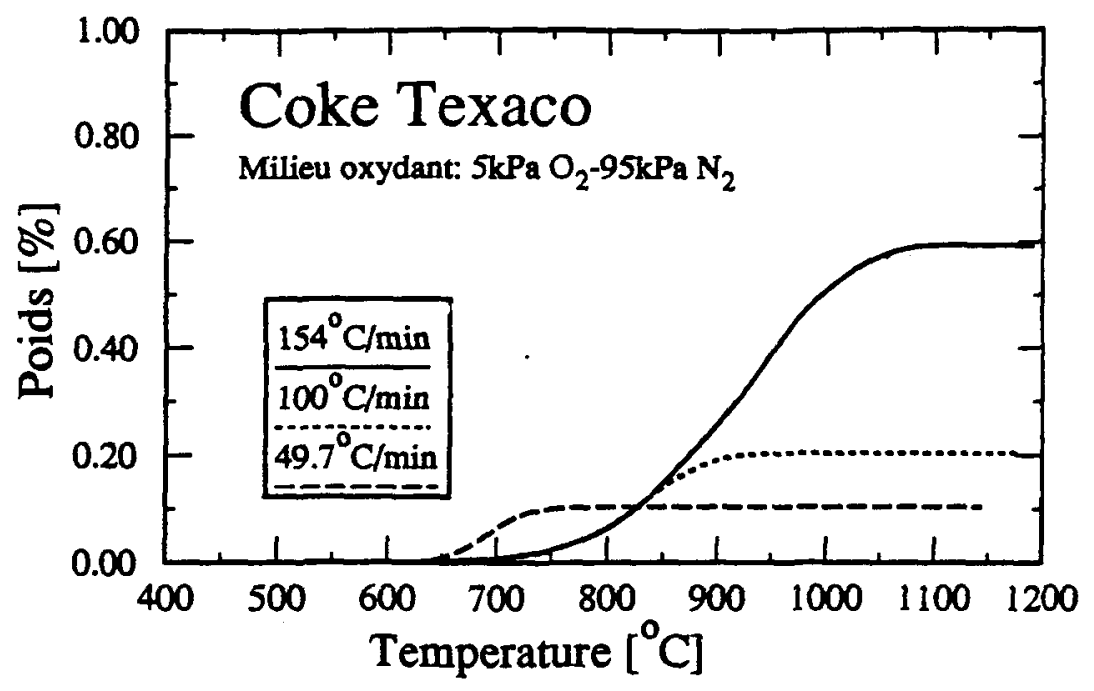

Fygure 6.30 : Effet du taux de chauffage sur l'hydrogène en milleu oxydant (95\% azote, $5 \%$ oxygène).

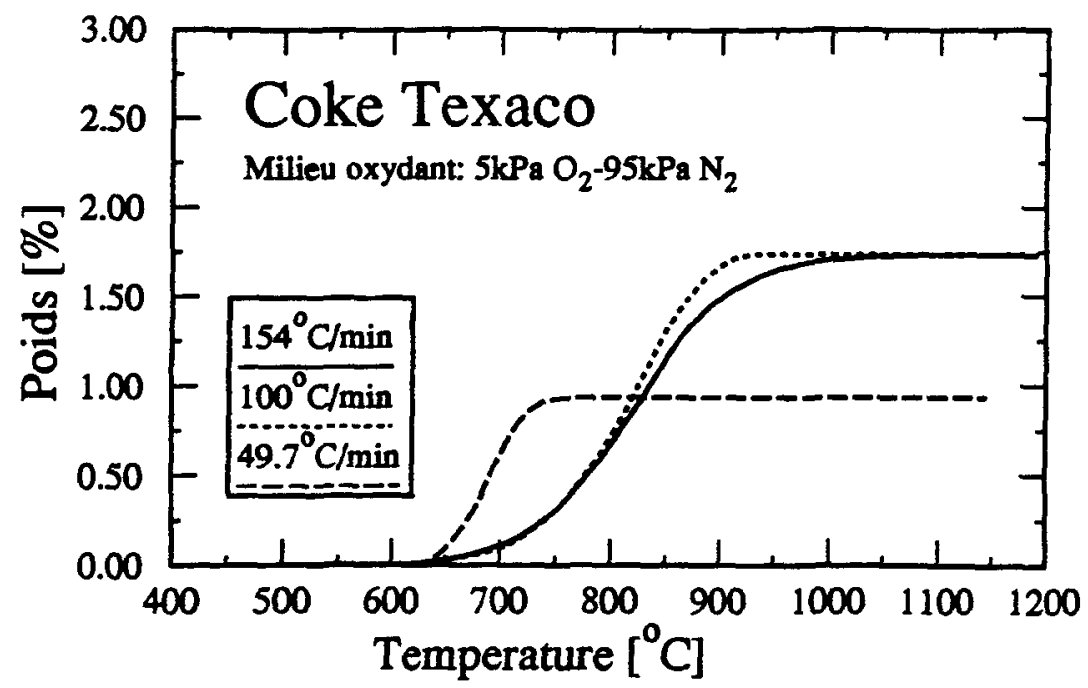

Fygure 6.31: Effet du taux de chauffage sur le méthane en milleu oxydant ( $95 \%$ azote, $5 \%$ oxygène). 


\section{VI.5.4 Estimation des quantités cumulatives d'hydrogène, de méthane et de coke consommées par la combustion en atmosphère oxydante}

La différence entre la courbe cumulative relative au produit dégagé en milieu neutre et la courbe relative au produit restant en milieu oxydant, donne une bonne approximation de la quantité d'hydrogène et de méthane consommée par la combustion au cours de la pyrolyse. Cette approximation est valable dans l'hypothèse où la dévolatilisation du coke vert n'est pas influencée par la nature du milieu. Bien que la comparaison des thermogrammes en milieux neutre et oxydant prouve que ce n'est pas tout à fait le cas lorsque le taux de chauffage est élevé, on peut quand même admettre que cette hypothèse est réaliste; étant donné que l'émission des produits volatils en milieu neutre a lieu dans le même intervalle de températures que la détection de ces mêmes volatils en présence d'air (se référer aux figures 6.24 à 6.26). Cette hypothèse est d'ailleurs soutenue par bon nombre d'auteurs $[10,11,14,15]$; de plus, elle constitue un moyen facile d'apprécier la combustion des produits de dévolatilisation au cours de la calcination sous différentes conditions.

Les figures 6.32 et 6.33 montrent l'influence du taux de chauffage sur le poids cumulatif d'hydrogène et de méthane ayant subi la combustion au cours de la pyrolyse du coke TEXACO en milieu oxydant. On constate que plus le chauffage est lent, plus grandes sont les quantités de produits légers ayant subi la combustion, à cause de la durée de l'expérience qui tient plus longtemps les volatils au contact de l'oxygène comburant $[7,22]$.

De plus, un chauffage rapide retarde la combustion des volatils légers en atmosphère oxydante, tout comme le taux de chauffage rapide retardait l'émission de ces mêmes volatils en atmosphère neutre [3,22].

En ce qui concerne l'hydrogène, on constate que la combustion n'est pas achevée à $1150^{\circ} \mathrm{C}$, en fin d'expérience. En effet, les expériences en milieu neutre démontrent que l'hydrogène s'échappe encore à cette température (voir figures 5.8 à 5.10). En ce qui concerne le méthane, on remarque que la combustion a lieu dans un intervalle de température plus restreint (entre 600 et $750^{\circ} \mathrm{C}$ ) lorsque le taux de chauffage est faible; à $100^{\circ} \mathrm{C} / \mathrm{min}$, cet intervalle s'élargit entre 600 et $850^{\circ} \mathrm{C}$, et entre 600 et 
$900^{\circ} \mathrm{C}$ à $150^{\circ} \mathrm{C} / \mathrm{min}$. Le palier présent ensuite à plus haute température montre que la combustion du méthane est bien achevée.

On remarque que l'intervalle pendant lequel le produit volatil brûle en atmosphère oxydante correspond exactement à l'intervalle d'émission de ce même produit en milieu neutre.

En annexe VI, on trouve les combustions pour les trois autres types de coke. Le tableau 6.2 donne le poids d'hydrogène, de méthane et de coke ayant subi la combustion au cours de la calcination des quatre types de coke, en atmosphère oxydante à $5 \%$ d'oxygène. Pour les cokes CONOCO et YPF, la quantité totale maximale d'hydrogène ou de méthane ayant subi la combustion au cours de la calcination est observée à $100^{\circ} \mathrm{C} / \mathrm{min}$. Des valeurs très proches sont relatées pour le coke $\mathrm{RTW}$ à $100^{\circ} \mathrm{C} / \mathrm{min}$ et à $50^{\circ} \mathrm{C} / \mathrm{min}$. La localisation de ces maxima en ce qui concerne la combustion des produits volatils est en accord avec la localisation des maxima constatés auparavant en ce qui concerne l'émission de ces mêmes produits en milieu neutre (se référer au tableau 5.2).

La figure 6.34 montre la quantité cumulative de coke ayant subi la combustion au cours de la pyrolyse à $50^{\circ} \mathrm{C} / \mathrm{min}$, pour les quatre types de coke. Cette valeur est obtenue soit par la différence entre les deux thermogrammes en milieux neutre et oxydant (figure 6.11), soit par la différence entre la courbe totale en milieu oxydant " $\mathrm{T}_{0}$ " et les trois courbes relatives aux produits $\mathrm{H}_{2}, \mathrm{CH}_{4}$ et condensables en milieu neutre " $H_{n}+M_{n}+C_{n}$ " (figure 6.35). Cette deuxième façon de procéder ramène toutes les courbes sur une même base à $450^{\circ} \mathrm{C}$. On constate que la combustion du coke n'a pas lieu à $100^{\circ} \mathrm{C} / \min$ parce que la différence entre les deux thermogrammes (figure 6.10) en milieux neutre et oxydant ramenés sur une même base est négligeable. De plus, bien que très faible, cette valeur reste constante au cours de la calcination, ce qui signifie qu'aucune perte de poids n'est engendrée par la combustion du coke, autrement dit que la combustion du coke n'a pas lieu dans ces conditions. D'autre part, on constate sur la figure 6.34 que la combustion du coke CONOCO, moins volatil, débute à partir de $600^{\circ} \mathrm{C}$, contrairement aux autres cokes plus volatils qui brûlent à partir de $700-750^{\circ} \mathrm{C}$ seulement. 
La figure 6.36 montre l'effet du taux de chauffage sur la combustion du résidu de coke CONOCO au cours de la calcination; rappelons que c'est le seul coke qui brûle à partir de $100^{\circ} \mathrm{C} / \mathrm{min}$. On constate que la combustion du résidu de coke ne fait que s'amorcer à partir de $100^{\circ} \mathrm{C} / \mathrm{min}$ (surtout entre 750 et $900^{\circ} \mathrm{C}$ ) mais elle ne devient significative qu'à partir de $50^{\circ} \mathrm{C} / \mathrm{min}$.

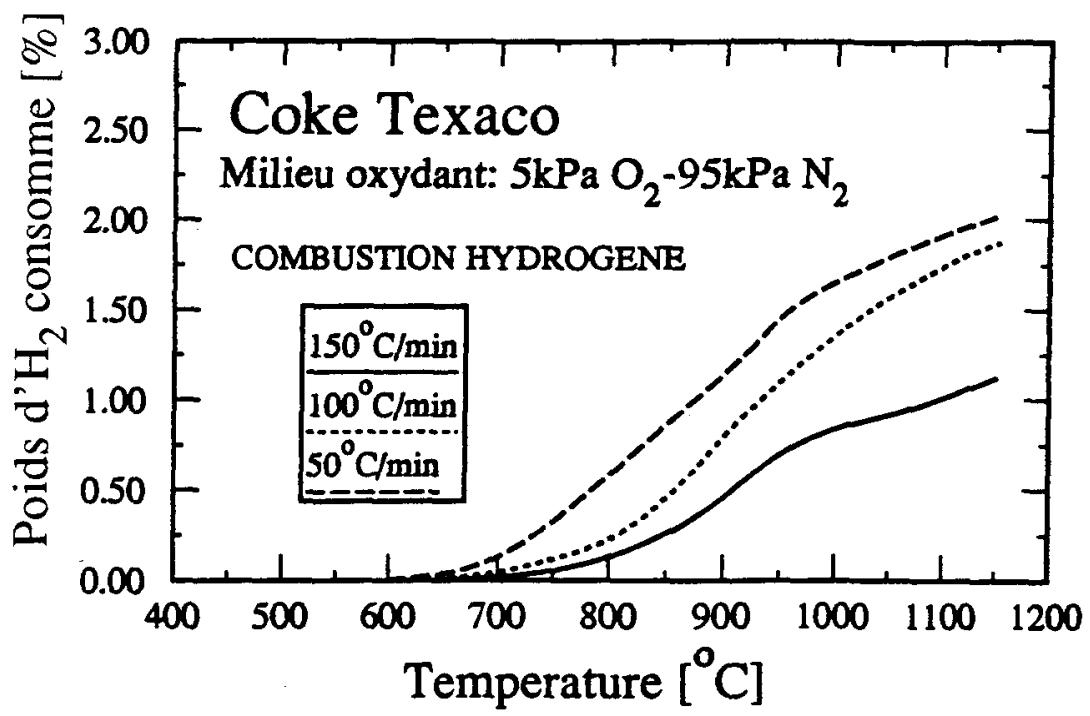

Flgure 6.32 : Effet du taux de chauffage sur la combustion de l'hydrogène du coke TEXACO, en milleu oxydant. 


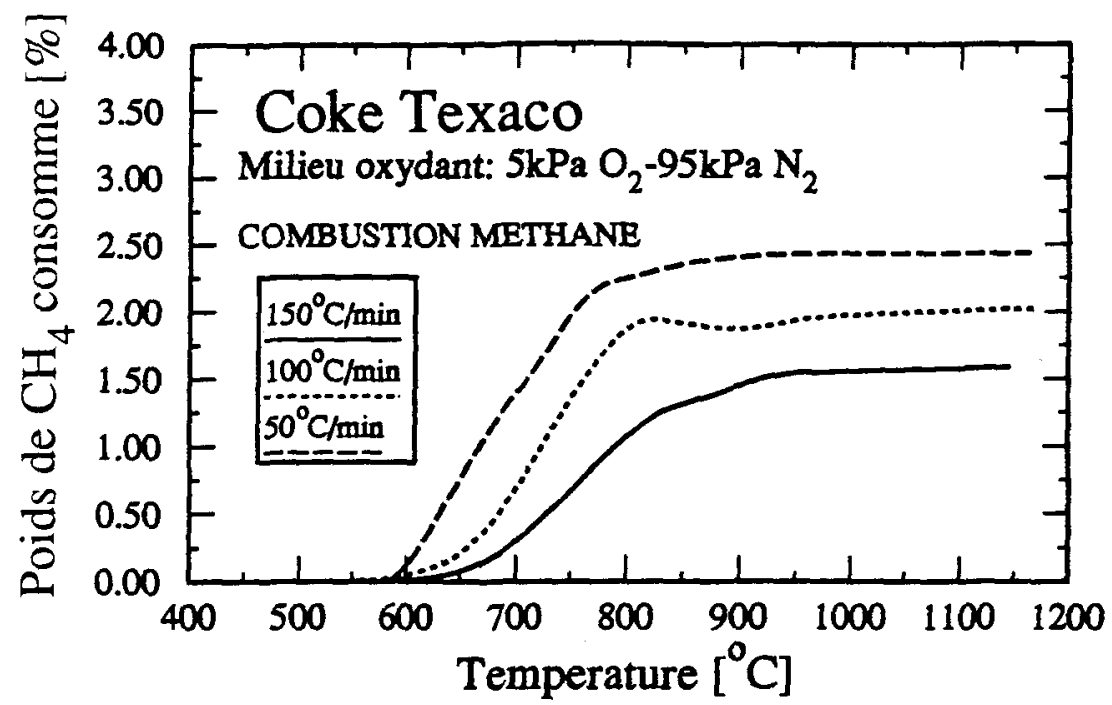

Figure 6.33 : Effet du taux de chauffage sur la combustion du méthane du coke TEXACO, en millieu oxydant.

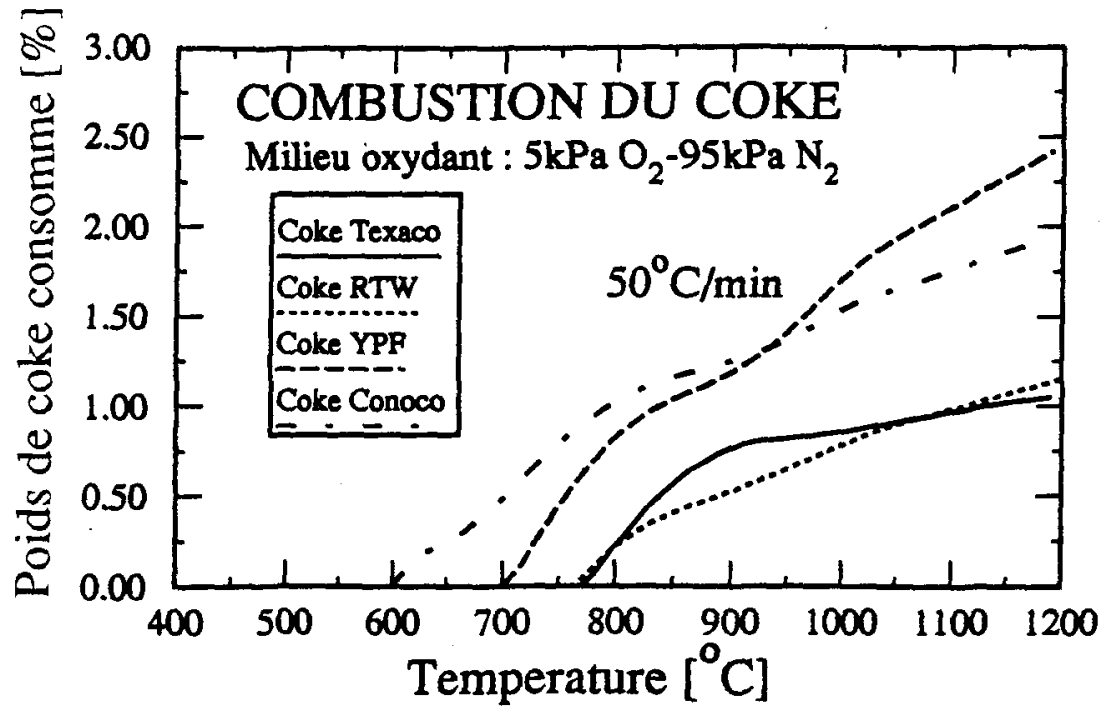

Flgure 6.34: Combustion des quatres types de coke à $50^{\circ} \mathrm{C} / \mathrm{min}$, en millieu oxydant ( $95 \%$ azote, $5 \%$ oxygène). 


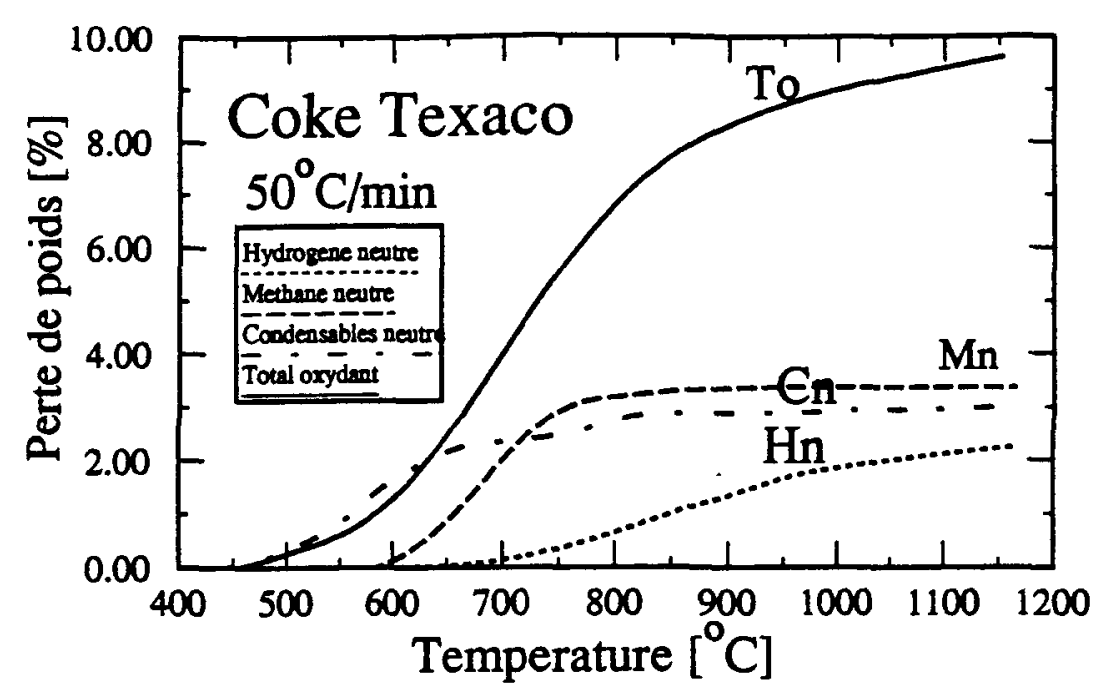

Flgure 6.35: Valeurs cumulatives de perte de poids, pour le calcul de la combustion du coke à $50^{\circ} \mathrm{C} / \mathrm{min}$.

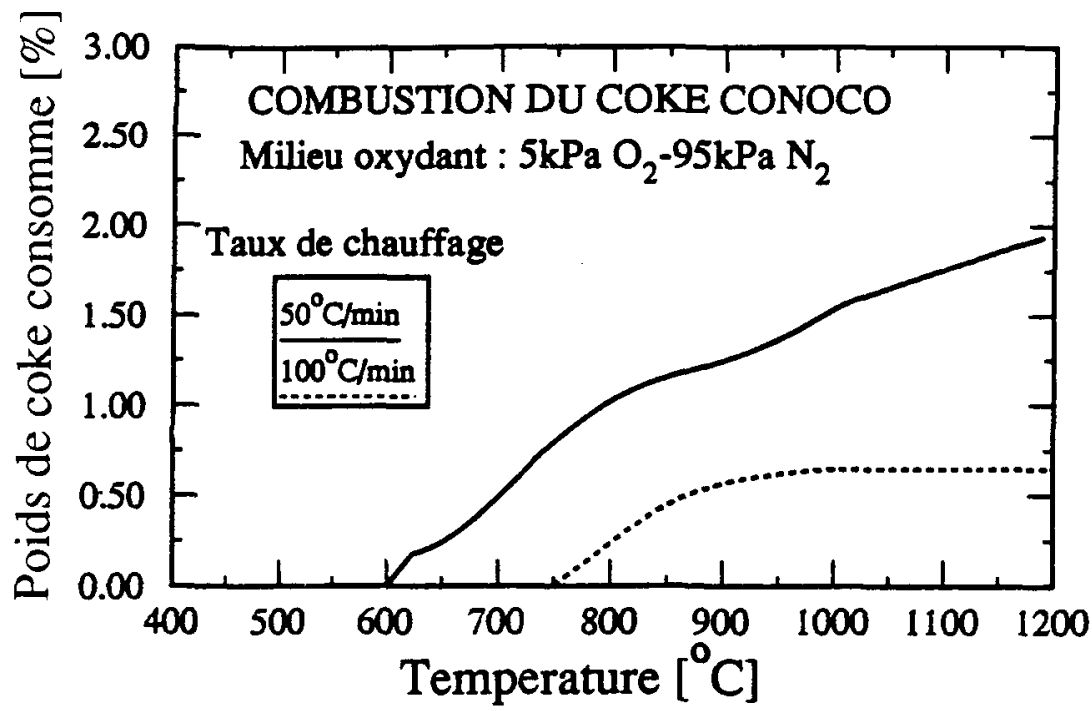

Figure 6.36 : Effet du taux de chauffage sur la combustion du coke CONOCO 
Tableau 6.2: $\quad$ Combustion des volatils et du coke en milieu oxydant $\left(5 \% \mathrm{O}_{2}-95 \% \mathrm{~N}_{2}\right)$.

\begin{tabular}{|c|c|c|c|c|}
\hline Cokes & $\begin{array}{l}\text { Taux de } \\
\text { chauffage } \\
{\left[^{\circ} \mathrm{C} / \text { min }\right]}\end{array}$ & $\begin{array}{c}\text { Hydrogène } \\
{[\%]}\end{array}$ & $\begin{array}{c}\text { Méthane } \\
{[\%]}\end{array}$ & $\begin{array}{c}\text { Résidu de } \\
\text { colke } \\
{[\%]}\end{array}$ \\
\hline TEXACO & $\begin{array}{r}154.0 \\
100.0 \\
49.7\end{array}$ & $\begin{array}{l}1.12 \\
1.88 \\
2.02\end{array}$ & $\begin{array}{l}1.59 \\
2.02 \\
2.43\end{array}$ & $\begin{array}{l}- \\
- \\
1.05\end{array}$ \\
\hline RTW & $\begin{array}{r}158.0 \\
101.0 \\
48.2\end{array}$ & $\begin{array}{l}1.57 \\
2.19 \\
2.21\end{array}$ & $\begin{array}{l}1.88 \\
1.99 \\
2.04\end{array}$ & $\begin{array}{l}- \\
- \\
1.15\end{array}$ \\
\hline YPF & $\begin{array}{r}152.9 \\
101.0 \\
49.1\end{array}$ & $\begin{array}{l}1.47 \\
2.30 \\
1.96\end{array}$ & $\begin{array}{l}1.73 \\
3.20 \\
1.40\end{array}$ & $\begin{array}{l}- \\
2.42\end{array}$ \\
\hline CONOCO & $\begin{array}{r}156.7 \\
96.6 \\
47.9\end{array}$ & $\begin{array}{l}1.61 \\
2.03 \\
1.87\end{array}$ & $\begin{array}{l}1.76 \\
2.02 \\
1.32\end{array}$ & $\begin{array}{l}- \\
0.65 \\
1.94\end{array}$ \\
\hline
\end{tabular}


VI.6 Effet de la pression partielle en oxygène dans le milieu réactionnel

Etant donné les difficultés expérimentales rencontrées à faible vitesse de chauffage, sous des pressions partielles en oxygène supérieures à $5 \mathrm{kPa}$ (détérioration rapide du creuset, explosion mineure), on se limitera à l'étude de l'influence du pourcentage d'oxygène dans le milieu à $150^{\circ} \mathrm{C} / \mathrm{min}$ uniquement.

\section{VI.6.1 Effet sur la courbe thermogravimétrique}

La figure 6.37 compare les thermogrammes obtenus à $150^{\circ} \mathrm{C} / \mathrm{min}$ avec le coke TEXACO, en atmosphère oxydante, sous différentes pressions partielles en oxygène: $3,5,7$ et $10 \mathrm{kPa}$. On constate que le pourcentage d'oxygène dans le milieu affecte très peu le thermogramme. Les thermogrammes obtenus avec 3 et $5 \mathrm{kPa}$ d'oxygène sont très proches et suivent la même évolution; il en est de même pour les thermogrammes obtenus avec 7 et $10 \mathrm{kPa}$ sur lesquels on peut remarquer un accroissement de la pente à haute température à partir de $7 \mathrm{kPa}$, à cause de la combustion du résidu carboné qui est amorcée à $150^{\circ} \mathrm{C} / \mathrm{min}$ lorsque le milieu contient un pourcentage plus élevé en oxygène. Rappelons qu'à $5 \mathrm{kPa}$ de pression partielle en oxygène dans le milieu, le coke n'entre pas en combustion (figure 6.9).

\section{VI.6.2 Effet sur les produits de la dévolatilisation: Hydrogène et méthane}

La figure 6.38 montre l'influence du pourcentage d'oxygène dans le milieu sur la concentration en $\mathrm{H}_{2}$ et $\mathrm{CH}_{4}$ au cours de la pyrolyse du coke TEXACO à $150^{\circ} \mathrm{C} / \mathrm{min}$. On constate qu'à partir de $7 \mathrm{kPa}$ de pression partielle en oxygène dans le milieu réactionnel, les produits volatils non consommés par la combustion sont en très faibles proportions, de l'ordre de quelques dizaines de ppm seulement pour 100 grammes de coke vert. Cette situation favorise l'attaque du résidu de coke par l'oxygène à haute température. 


\section{VI.6.3 Effet sur les gaz: Monoxyde et dioxyde de carbone}

La figure 6.39 montre l'influence du pourcentage d'oxygène sur la formation du monoxyde et du dioxyde de carbone, au cours de la pyrolyse du coke TEXACO à $150^{\circ} \mathrm{C} / \mathrm{min}$. On constate que la présence d'oxygène moléculaire en quantité plus importante dans le milieu, favorise la formation de $\mathrm{CO}_{2}$, au détriment du $\mathrm{CO}$ qui se forme en quantitê beaucoup moindre. Le monoxyde de carbone se forme dans tous les cas avant le dioxyde qui se forme à plus haute température. A $7 \mathrm{kPa}$ d'oxygène dans le milieu réactionnel, les quantités maximales de $\mathrm{CO}$ et de $\mathrm{CO}_{2}$ sont quasiment égales et décalées de $50^{\circ} \mathrm{C}$; le maximum se situe à $800^{\circ} \mathrm{C}$ pour le $\mathrm{CO}$ et à $850^{\circ} \mathrm{C}$ pour le $\mathrm{CO}_{2}$.

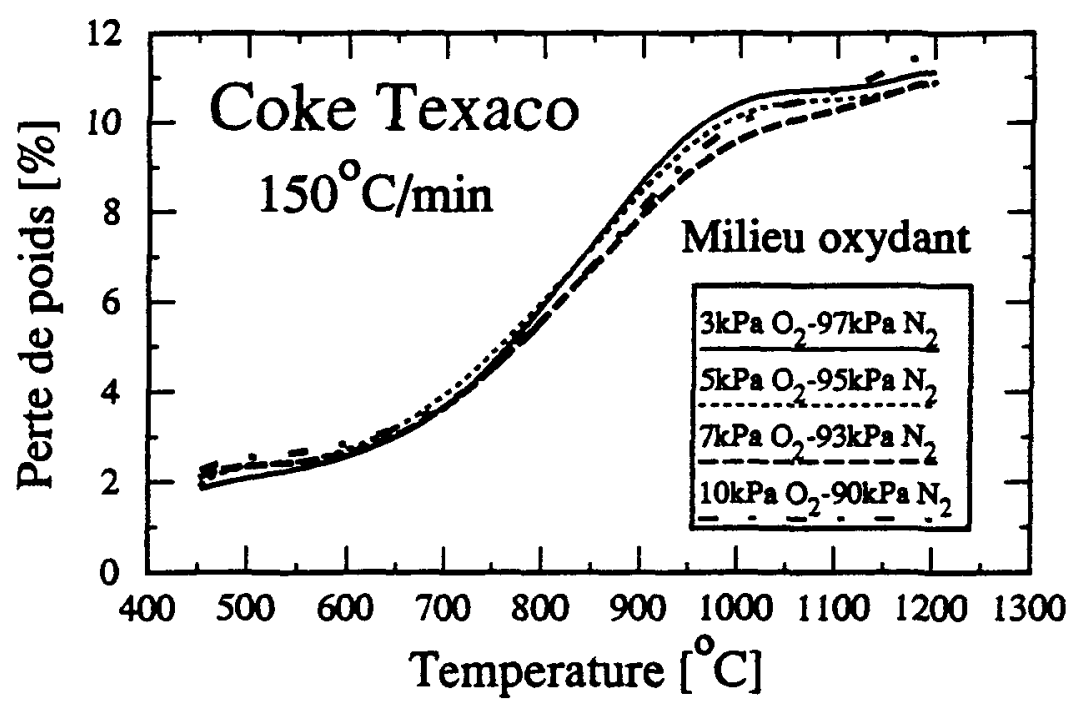

Figure 6.37 : Effet de la pression partielle en oxygène dans le milieu sur le thermogramme, pour le coke TEXACO à $150^{\circ} \mathrm{C} / \mathrm{min}$. 


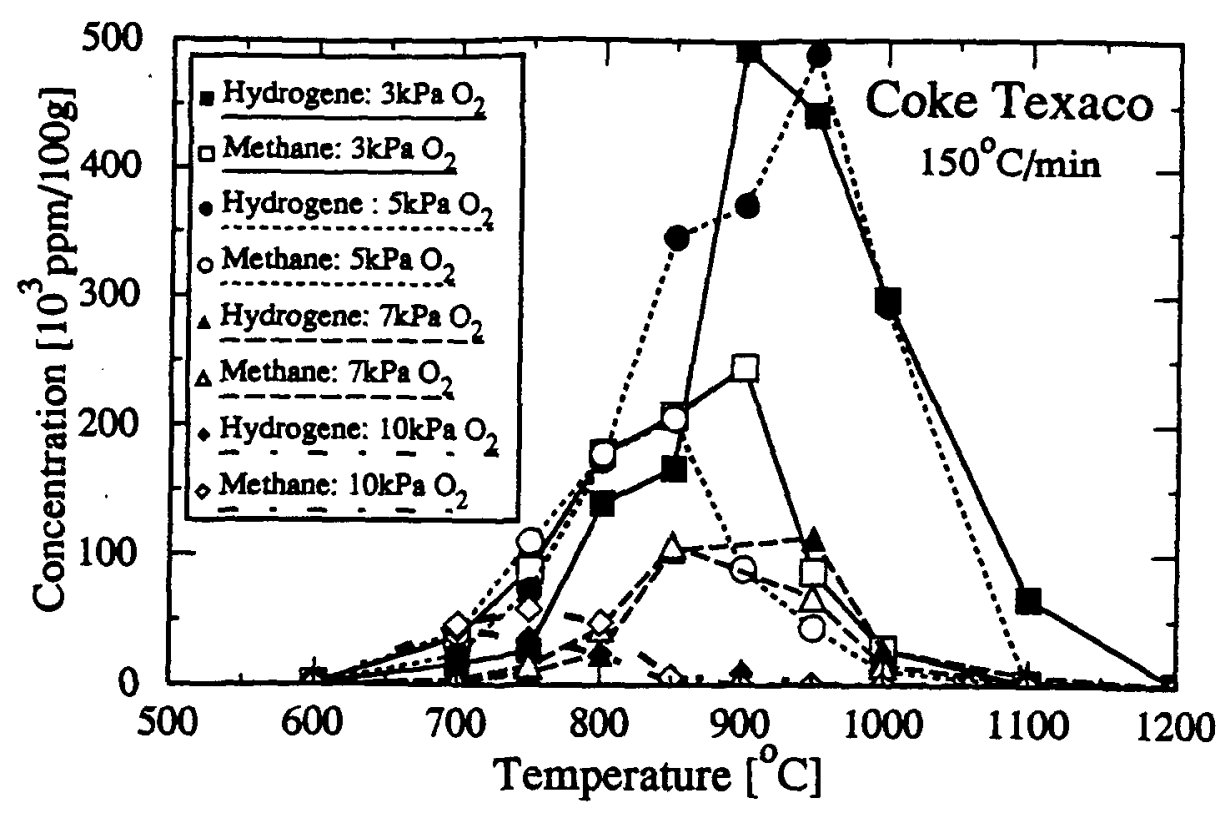

Figure 6.38 : Effet de la pression partielle en oxygène dans le milieu sur l'hydrogène et le méthane, pour le coke TEXACO à $150^{\circ} \mathrm{C} / \mathrm{min}$.

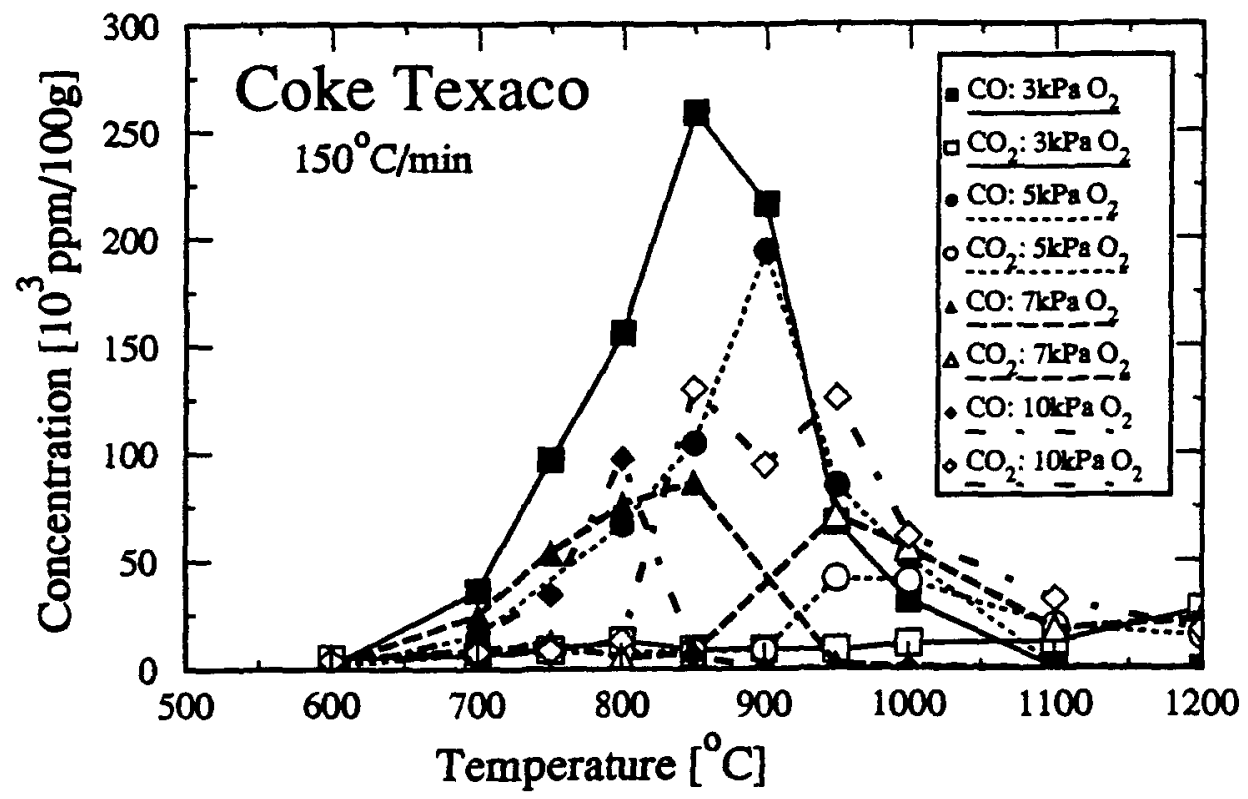

Figure 6.39 : Effet de la pression partielle en oxygène dans le milieu sur les monoxyde et dioxyde de carbone, pour le coke TEXACO à $150^{\circ} \mathrm{C} / \mathrm{min}$. 


\section{VI.7 Calculs d'équilibre}

En se basant sur l'hypothèse que la dévolatilisation du coke vert ne dépend pas du milieu réactionnel dans lequel elle a lieu à faible taux de chauffage, un calcul des concentrations à l'équilibre des composés entrant en jeu a été réalisé. Le principe est de mettre les produits de la dévolatilisation du coke en milieu neutre, en présence d'oxygène, dans des conditions bien précises de température et de pression, afin de pouvoir simuler l'état du système au cours du traitement thermique de calcination. Ces calculs sont réalisés à l'aide du logiciel commercial "OUTOKUMPU HSC version 1.10". Ils donnent une bonne idée de l'état du système, en comparant les résultats obtenus à l'équilibre par calculs thermodynamiques, avec les résultats expérimentaux obtenus en milieu oxydant. Dans le cas où les concentrations à l'équilibre données par le logiciel sont proches des concentrations expérimentales, on dira que les produits volatils entrent instantanément en combustion avec l'oxygène du milieu et que la cinétique est quasiment inexistante. Dans le cas contraire, on dira que la cinétique de combustion des volatils au cours de la calcination du coke vert en atmosphère oxydante est bien réelle.

La comparaison est faite pour deux taux de chauffage: $150^{\circ} \mathrm{C} / \mathrm{min}$ et $50^{\circ} \mathrm{C} / \mathrm{min}$; même si l'hypothèse de base ne s'applique pas tout à fait à $150^{\circ} \mathrm{C} / \mathrm{min}$ à cause du décalage observé dans les thermogrammes en milieux oxydant et neutre (figure 6.9). Néanmoins, l'analyse des gaz montre que les gaz de dévolatilisation (hydrogène et méthane) se retrouvent dans le même intervalle de températures dans les deux milieux (figure 6.24).

Le mélange initial est constitué de deux phases: une phase gazeuse comprenant l'hydrogène, le méthane, le goudron et le monoxyde de carbone dans un milieu contenant $5 \%$ d'oxygène, le reste étant l'azote; et une phase solide composée du résidu de carbone solide (à haute température uniquement). Les concentrations initiales des produits volatils, issues des expériences menées en atmosphère neutre, sont ramenées en fractions molaires dans la phase gazeuse, en supposant un débit constant de $10.6 \mathrm{~L} / \mathrm{min}$. Un exemple de mélange est donné dans le tableau 6.3 , pour le coke Texaco à $150^{\circ} \mathrm{C} / \mathrm{min}$. On remarque sur ce tableau que le goudron, dont 
la forme la plus représentative est le $\mathrm{C}_{18} \mathrm{H}_{12}$ est introduit sous forme de deux composés organiques: le $\mathrm{C}_{2} \mathrm{H}_{6(\mathrm{~g})}$ et le $\mathrm{C}_{(\mathrm{g})}$. En effet, le logiciel ne permettant pas le traitement du composé aromatique $\mathrm{C}_{18} \mathrm{H}_{12}$, et étant donné que ce composé est instable à partir de $600^{\circ} \mathrm{C}$, il a été converti en $\mathrm{C}_{2}$ $\mathrm{H}_{6(\mathrm{~g})}$ et $\mathrm{C}_{(\mathrm{g})}$ selon la transformation suivante:

$$
\mathrm{C}_{18} \mathrm{H}_{12(\mathrm{~g})}-\cdots------>\quad 2 \mathrm{C}_{2} \mathrm{H}_{6(\mathrm{~g})}+14 \mathrm{C}_{(\mathrm{g})}
$$

Les calculs d'équilibre sont basés sur la minimisation de l'énergie de Gibbs pour toutes les réactions possibles, dans des conditions de température et de pression précisées au préalable au début de la simulation. Les résultats sont donnés en nombre de moles et convertis en fraction molaire dans chacune des phases.

Tableau 6.3: $\quad$ Composition du mélange pour le calcul des concentrations à l'équilibre, pour le coke TEXACO à $150^{\circ} \mathrm{C} / \mathrm{min}$.

\begin{tabular}{|c|c|c|c|c|c|c|}
\hline Température & $600^{\circ} \mathrm{C}$ & $700^{\circ} \mathrm{C}$ & $800^{\circ} \mathrm{C}$ & $900^{\circ} \mathrm{C}$ & $1000^{\circ} \mathrm{C}$ & $1200^{\circ} \mathrm{C}$ \\
\hline \multirow{2}{*}{\multicolumn{7}{|c|}{$\begin{array}{c}\text { Phase gazeuse } \\
{[\%]}\end{array}$}} \\
\hline & & & & & & \\
\hline $\mathrm{H}_{2}(\mathrm{~g})$ & 0.0097 & 0.47 & 3.07 & 9.52 & 9.41 & 3.98 \\
\hline $\mathrm{CH}_{4}$ (g) & 0.020 & 0.96 & 3.14 & 2.15 & 0.31 & 0.02 \\
\hline $\mathrm{CO}(\mathrm{g})$ & 0.0038 & 0.24 & 0.32 & 2.06 & 0.48 & 0.00 \\
\hline $\mathrm{O}_{2}(\mathrm{~g})$ & 5 & 5 & 5 & 5 & 5 & 5 \\
\hline $\mathrm{N}_{2}(\mathrm{~g})$ & 92.74 & 89.50 & 86.87 & 81.27 & 84.27 & 90.99 \\
\hline $\mathrm{CO}_{2}(\mathrm{~g})$ & 0 & 0 & 0 & 0 & 0 & 0 \\
\hline $\mathrm{H}_{2} \mathrm{O}(\mathrm{g})$ & 0 & 0 & 0 & 0 & 0 & 0 \\
\hline $\mathrm{C}_{2} \mathrm{H}_{6}(\mathrm{~g})$ & 0.28 & 0.48 & 0.20 & 0 & 0 & 0 \\
\hline $\mathrm{C}(\mathrm{g})$ & 1.95 & 3.35 & 1.40 & 0 & 0 & 0 \\
\hline \multicolumn{7}{|l|}{$\frac{\text { Phase solide }^{(*)}}{[\%]}$} \\
\hline $\mathrm{C}(\mathrm{s})$ & 0 & 0 & 0 & 0 & 0 & 100 \\
\hline
\end{tabular}


(*) : Pas de phase solide de coke présente à $150^{\circ} \mathrm{C} / \mathrm{min}$ puisque la combustion du coke dans un milieu contenant $5 \%$ d'oxygène ne débute qu'à partir de $50^{\circ} \mathrm{C} / \mathrm{min}$.

\section{VI.7.1 Analyse des concentrations à l'équilibre}

\section{VI.7.1.A A haut taux de chauffage: $150^{\circ} \mathrm{C} / \mathrm{min}$}

Les figures 6.40 à 6.44 comparent les concentrations dans le mélange initial (input), les concentrations à l'équilibre données par le logiciel (output-équilibre) et les concentrations expérimentales (output-expérience), et ce pour les composés d'intérêt suivants: l'hydrogène, le méthane, les monoxyde et dioxyde de carbone et l'eau. L'azote, neutre, reste inchangé car il ne participe à aucune réaction.

$\mathrm{Au}$ vu de ces figures et bien que les réactions entrant en jeu soient nombreuses et complexes, il se dégage certaines tendances concernant le comportement des composés conduisant à l'équilibre. Ces tendances peuvent être résumées comme suit:

Il y a formation d'hydrogène entre 600 et $850^{\circ} \mathrm{C}$; par contre après $850^{\circ} \mathrm{C}$, l'hydrogène entre en combustion avec l'oxygène. Or, c'est précisément dans cet intervalle de température (entre 600 et $850^{\circ} \mathrm{C}$ ) que le méthane et le goudron se trouvent en quantités importantes dans le milieu; de plus on constate sur la figure 6.41 que la totalité du méthane est consommée. On peut donc penser que le méthane et le goudron réagissent avec l'oxygène pour former de l'hydrogène entre autre, mais également du $\mathrm{CO}$ et de l'eau (figures 6.42 et 6.44). A partir de $900^{\circ} \mathrm{C}$, les quantités de méthane et de goudron diminuent très significativement dans le milieu et l'hydrogène devient le composé dominant. Sa combustion donne des quantités importantes d'eau surtout à $1000^{\circ} \mathrm{C}$ (figure 6.44); le pic d'eau se produisant à la même température que le pic d'hydrogène observé sur la figure 6.40. Dès que la phase solide de carbone est présente (lorsque les volatils ne protègent plus la particule solide de coke), la combustion de l'hydrogène s'arrête au profit de celle du carbone solide sous forme de $\mathrm{CO}$. 


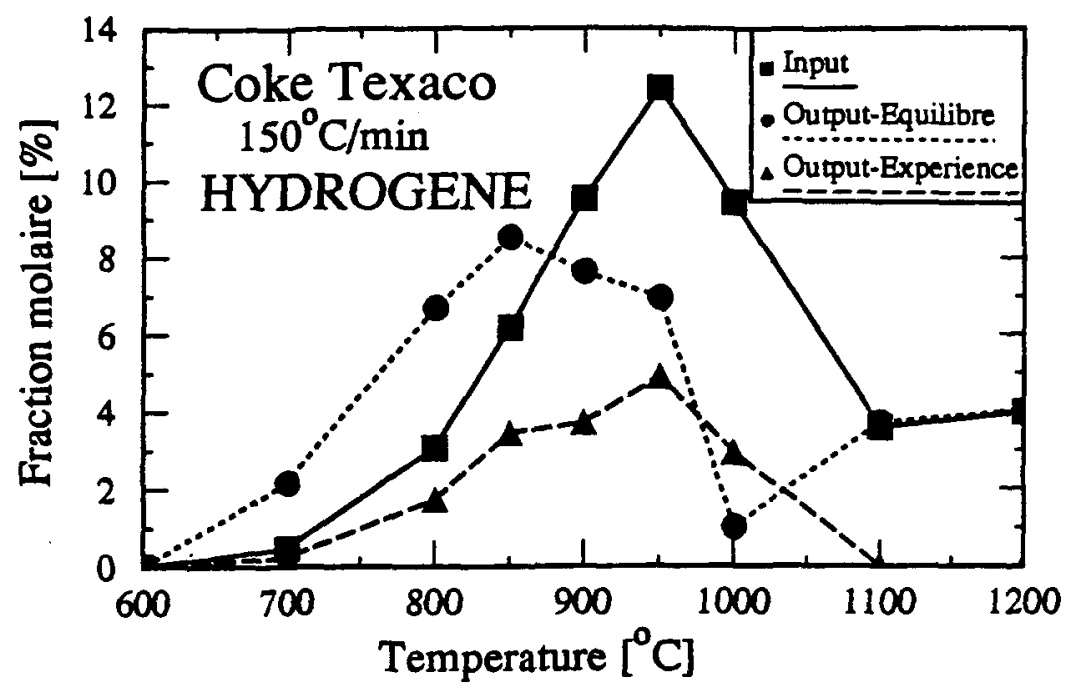

Flgure 6.40 : Comparaison de l'équillbre avec l'expérience pour l'hydrogène.

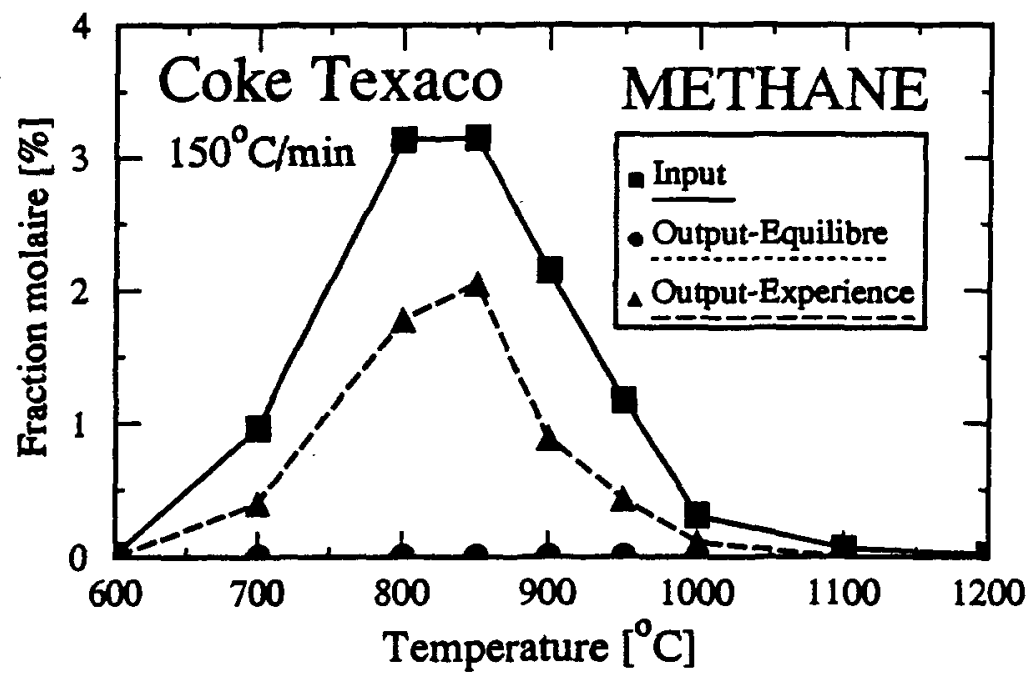

Flgure 6.41 : Comparaison de l'équilibre avec l'expérience pour le méthane. 


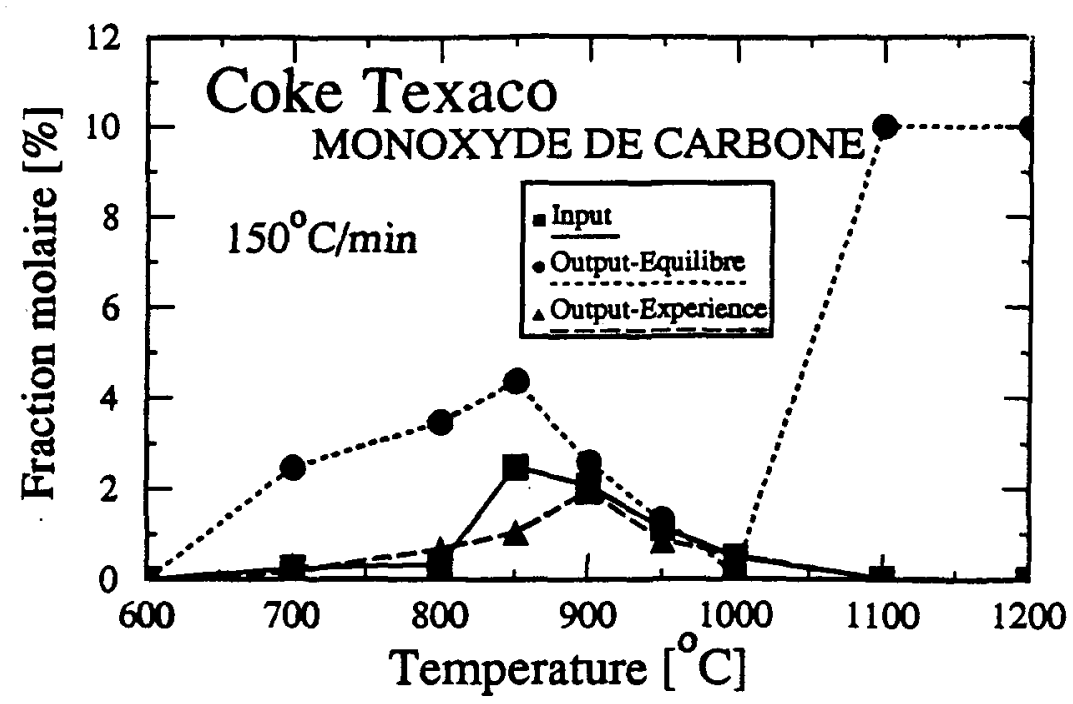

Figure 6.42: Comparaison de l'équilibre avec l'expérience pour le monoxyde de carbone.



Figure 6.43: Comparaison de l'équilibre avec l'expérience pour le dioxyde de carbone. 


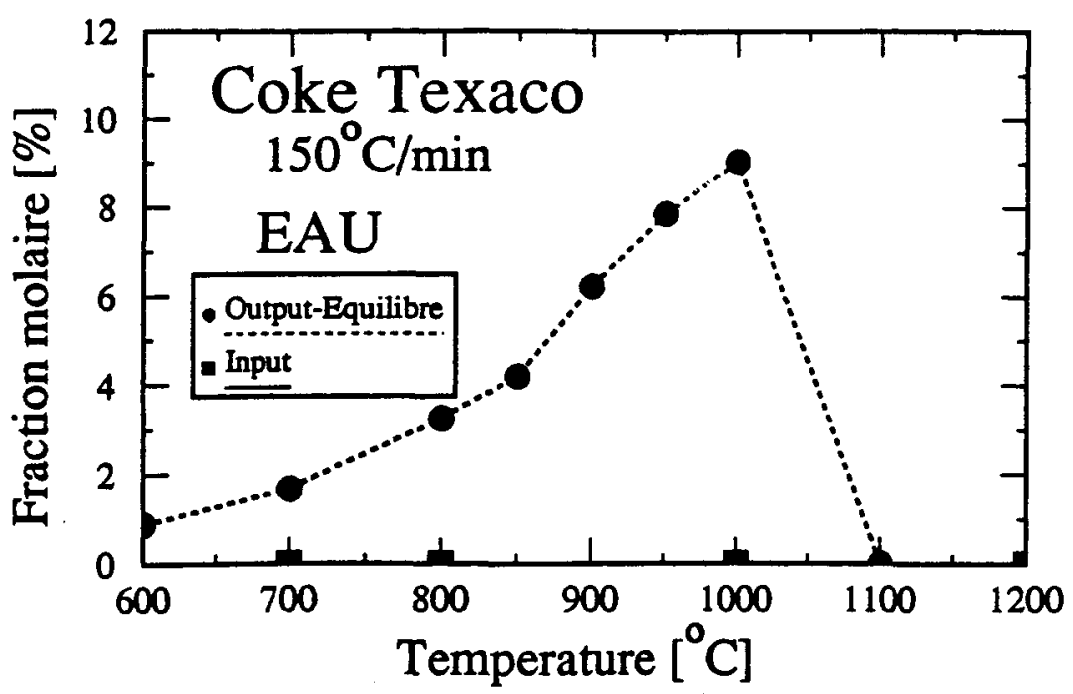

Figure 6.44: Comparaison de l'équilibre avec l'expérience pour l'eau.

\section{VI.7.1.B A faible taux de chauffage: $50^{\circ} \mathrm{C} / \mathrm{min}$}

Les résultats obtenus pour le coke TEXACO à $50^{\circ} \mathrm{C} / \mathrm{min}$ se trouvent en annexe VII.

En conclusion, on peut dire que:

1- la totalité du méthane et du goudron brülent dans le premier stade de la calcination;

2- l'oxygène réagit ensuite avec l'hydrogène et ce aussi longtemps que la phase solide de carbone est inexistante;

3- dès que la phase solide de carbone est présente, la combustion de l'hydrogène s'arrête au profit de celle du carbone.

\section{V.7.2 Comparaison des résultats expérimentaux avec l'équilibre}

Au cours des expériences de pyrolyse en milieu oxydant, l'état d'équilibre n'est jamais atteint et ce pour plusieurs raisons. D'une part, l'équilibre suppose que l'on laisse les composés participant aux réactions en présence pendant un certain temps jusqu'au moment où cet équilibre est 
atteint; or dans les expériences, l'accroissement de la température au cours de la calcination restreint de manière significative ce laps de temps. D'autre part, les calculs d'équilibre ne tiennent pas compte de la forme de la phase solide ni de la diffusion de l'oxygène pour atteindre cette phase; alors qu'au cours des expériences cet aspect a toute son importance.

La cinétique de combustion des produits volatils de dégazéification est donc bien réelle au cours de la calcination du coke vert en milieu oxydant. Les figures 6.32 et 6.33 en témoignent également. Cette constatation va à l'encontre du modèle de Borghi qui prétend qu'au cours de la calcination en atmosphère oxydante, les volatils entrent instantanément en réaction [10].

\section{VI.8 Conclusions}

La présence d'oxygène dans le milieu retarde la dévolatilisation, à haut taux de chauffage seulement $\left(150\right.$ et $\left.100^{\circ} \mathrm{C} / \mathrm{min}\right)$. Par contre, à $50^{\circ} \mathrm{C} / \mathrm{min}$, la dévolatilisation n'est pas influencée par la nature du milieu; toutefois, à partir de $750^{\circ} \mathrm{C}$ le résidu de coke brûle par combustion avec l'oxygène. Avant cette température ou lorsque le taux de chauffage est plus élevé (100 et $150^{\circ} \mathrm{C} / \mathrm{min}$ ), les volatils dégagés par la dévolatilisation constituent une protection autour de la particule, empêchant ainsi sa combustion $[7,13,14,22]$. Cette affirmation est d'ailleurs confirmée par l'analyse des gaz: hydrogène et méthane encore présents dans le milieu.

L'augmentation de la pression partielle en oxygène dans le milieu n'a pas une influence très significative sur le thermogramme à $150^{\circ} \mathrm{C} / \mathrm{min}$, sauf à haute température (à partir de $1100^{\circ} \mathrm{C}$ ) où la combustion du coke est entamée à partir de $7 \mathrm{kPa}$ de pression partielle en oxygène dans le milieu.

Au cours de la calcination du coke vert, un chauffage lent et une disponibilité accrue en oxygène dans le milieu facilite la combustion des produits volatils légers de type hydrogène et méthane. Ainsi, la cinétique de combustion des produits volatils est bien réelle comme le démontre les calculs d'équilibre. De plus, la combustion subséquente du résidu de coke 
est favorisée par un chauffage lent, un indice faible en matières volatiles du coke [7] ou encore une pression partielle élevée en oxygène dans le milieu. 


\section{CHAPITRE VII}

\section{MODELES CINETIgUES GLOBAUX DE DEVOLATILISATION ET DE COMBUSTION DU COKE VERT}

\section{VII.1 Introduction}

Un modèle cinétique global basé sur l'analyse thermogravimétrique non isotherme $[3,6,19]$ est proposé pour caractériser la dévolatilisation des quatre types de coke vert, en atmosphères oxydante et neutre, à 150 et $100^{\circ} \mathrm{C} / \mathrm{min}$ (lorsque la combustion du coke est inexistante en présence d'oxygène). Il permet de déterminer l'influence du milieu sur les paramètres cinétiques tels que l'ordre de la réaction, l'énergie d'activation et le facteur pré-exponentiel. Les résultats sont présentés dans le paragraphe VII.2 pour le coke TEXACO, les autres cokes figurant en annexe VIII.

Un modèle cinétique global est proposé pour la combustion du coke vert, en atmosphère oxydante à $50^{\circ} \mathrm{C} / \mathrm{min}$. les résultats se trouvent détaillés dans le paragraphe VII.3 pour les quatre types de coke.

\section{VII.2 Modèle cinétique global de dévolatilisation}

Le modèle cinétique est basé l'expression générale de la vitesse de perte de poids d'un solide au cours de sa pyrolyse (se référer au paragraphe I.5.2. A du chapitre I) $[3,6,5,19]$ :

$$
\mathrm{dX} / \mathrm{dt}=\mathrm{A} \exp ^{(-\mathrm{E} / \mathrm{RT})}(1-\mathrm{X})^{\mathrm{n}}
$$

Les paramètres cinétiques $\mathrm{E}$ (énergie d'activation) et $\mathrm{A}$ (facteur préexponentiel sont déduits graphiquement à partir des résultats expérimentaux, après avoir fixé l'ordre de la réaction $n$. Les résultats sont 
présentés sous la forme d'un diagramme d'Arrhénius portant (lnk) en fonction de $(1 / T)$ en graphique.

Les valeurs expérimentales sont les suivantes:

$\ln \mathrm{k}_{\exp }=\ln \left[(\mathrm{dX} / \mathrm{dt}) /(1-\mathrm{X})^{\mathrm{n}}\right]$

où $X$ représente la fraction de perte de poids issue de l'expérience de calcination.

Les valeurs issues du modèle sont les suivantes:

$\ln \mathrm{k}_{\bmod }=\ln \mathrm{A}-(\mathrm{E} / \mathrm{RT})$

où $\mathrm{A}$ et $\mathrm{E}$ sont les paramètres cinétiques calculés d'après le modèle.

Les figures 7.1 et 7.2 comparent les résultats obtenus en milieu neutre et en milieu oxydant à 5\% d'oxygène, pour le coke TEXACO à 150 et $100^{\circ} \mathrm{C} / \mathrm{min}$ respectivement. Les paramètres cinétiques globaux de dévolatilisation sont donnés dans le tableau 7.1 pour les quatre types de coke. Le diagramme d'Arrhénius pour les trois autres cokes est donné en annexe VIII.

L'ordre de la réaction n est fixé à 2 pour 100 et $50^{\circ} \mathrm{C} / \mathrm{min}$, et à 1.5 pour le taux de chauffage de $150^{\circ} \mathrm{C} / \mathrm{min}$. Quant à l'énergie d'activation $\mathrm{E}$, on constate que la présence d'oxygène dans le milieu accroît l'énergie; cette tendance est observée pour les quatre types de coke (tableau 7.1), exception faite du coke YPF à $100^{\circ} \mathrm{C} / \mathrm{min}$. 


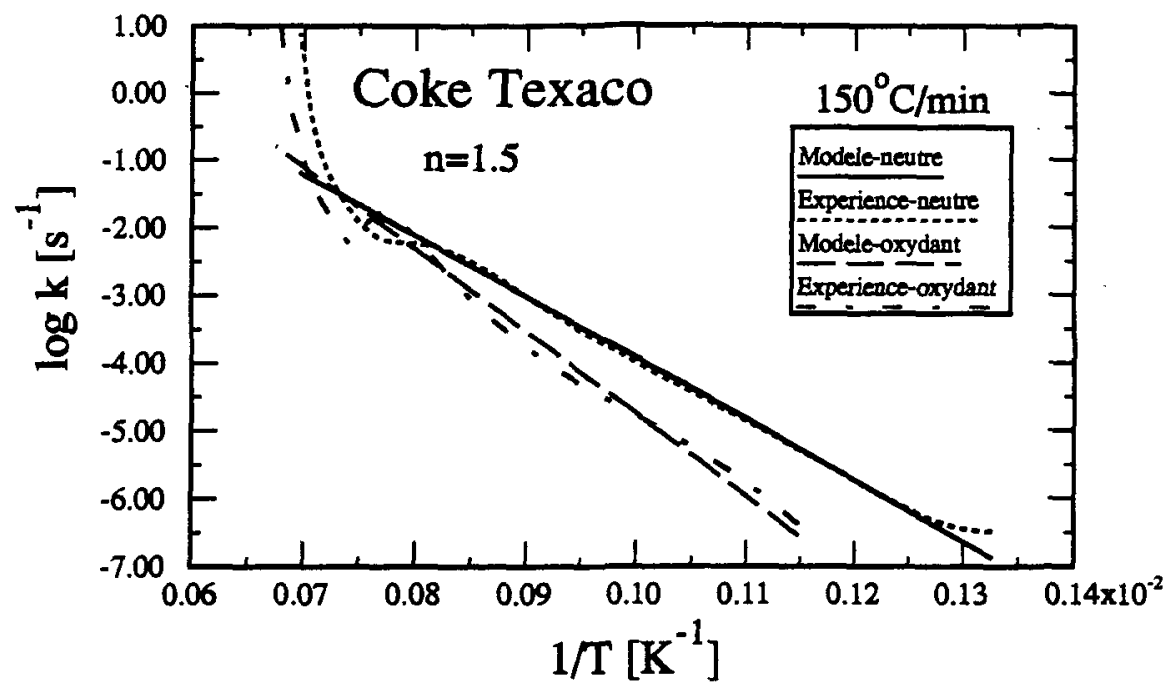

Figure 7.1: Cinétique de dévolatilisation du coke TEXACO à $150^{\circ} \mathrm{C} / \mathrm{min}$, milieux neutre et oxydant.

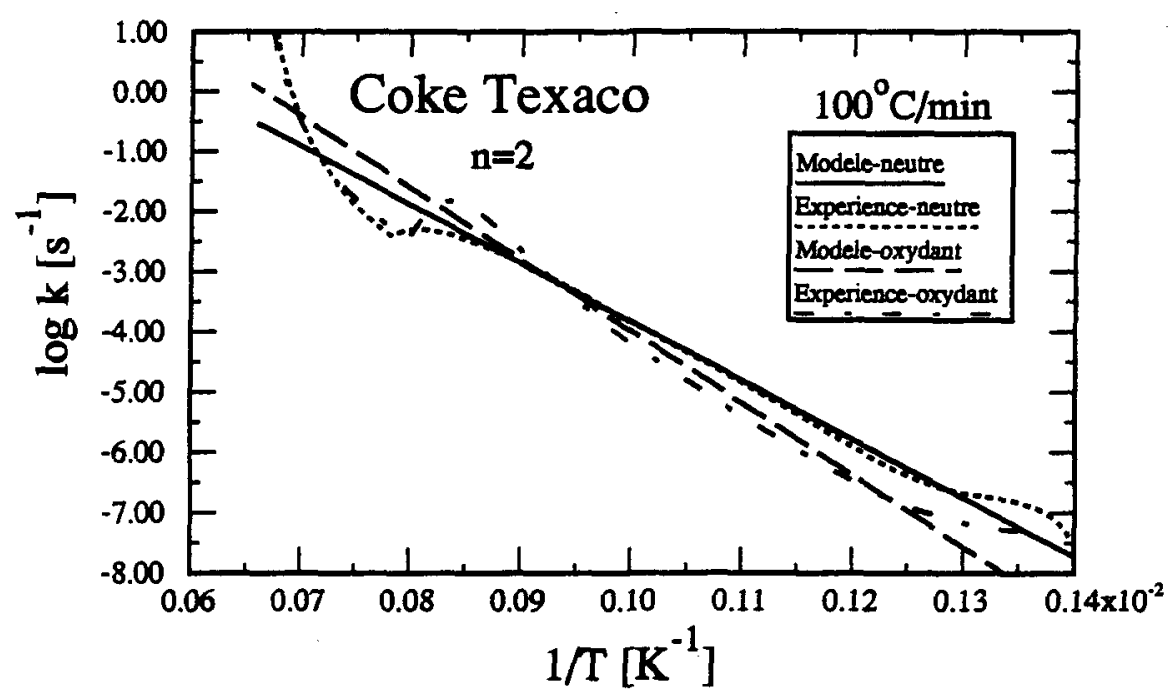

Figure 7.2: Cinétique de dévolatilisation du coke TEXACO à $100^{\circ} \mathrm{C} / \mathrm{min}$, milieux neutre et oxydant. 
Tableau 7.1: Paramètres cinétiques globaux de dévolatilisation en milieux neutre et oxydant pour les quatres types de cokes: TEXACO (a), RTW (b), YPF (c) et CONOCO (d)

\begin{tabular}{|c|c|c|c|}
\hline \multicolumn{4}{|c|}{ COKE TEXACO } \\
\hline $\begin{array}{l}\text { Taux de } \\
\text { chauffage } \\
{ }^{\circ} \mathrm{C} / \mathrm{min} \mid\end{array}$ & $\begin{array}{c}\text { Milieu neutre } \\
\begin{array}{c}\left(100 \% \mathrm{~N}_{2}\right) \\
\mathrm{E}\left[\mathrm{J} / \mathrm{mol}^{-1}\right] \\
\mathrm{A}\left[\mathrm{s}^{-1}\right]\end{array}\end{array}$ & $\begin{array}{l}\text { Taux de } \\
\text { chauffage } \\
{\left[^{\circ} \mathrm{C} / \mathrm{min}\right]}\end{array}$ & $\begin{array}{c}\text { Milieu oxydant } \\
\left(95 \% \mathrm{~N}_{2}-5 \% \mathrm{O}_{2}\right) \\
\mathrm{E}\left[\mathrm{J} / \mathrm{mol}^{-1]}\right. \\
\mathrm{A}\left[\mathrm{s}^{-1}\right]\end{array}$ \\
\hline 153.2 & $\begin{array}{l}n=1.5 \\
E=75056 \\
A=163\end{array}$ & 154 & $\begin{array}{l}n=1.5 \\
E=101074 \\
A=1638\end{array}$ \\
\hline 98.5 & $\begin{array}{l}n=2 \\
E=80916 \\
A=361\end{array}$ & 100 & $\begin{array}{l}n=2 \\
E=99019 \\
A=2732\end{array}$ \\
\hline 48.7 & $\begin{array}{l}n=2 \\
E=80457 \\
A=201\end{array}$ & $(*)$ & \\
\hline
\end{tabular}

(*) : Combustion des cokes TEXACO, RTW et YPF à partir de $50^{\circ} \mathrm{C} / \mathrm{min}$.

\begin{tabular}{|c|c|c|c|}
\hline \multicolumn{4}{|c|}{ COKE RTW } \\
\hline $\begin{array}{c}\text { Taux de } \\
\text { chauffage } \\
{\left[{ }^{\circ} \mathrm{C} / \mathrm{m} m\right]}\end{array}$ & $\begin{array}{c}\text { Milieu neutre } \\
\left(100 \% \mathrm{~N}_{2}\right) \\
\mathrm{E}\left[\mathrm{J} / \mathrm{mol}^{-}\right] \\
\mathrm{A}\left[\mathrm{s}^{-1}\right]\end{array}$ & $\begin{array}{l}\text { Taux de } \\
\text { chauffage } \\
{\left[^{\circ} \mathrm{C} / \mathrm{min}\right]}\end{array}$ & $\begin{array}{c}\text { Milieu oxydant } \\
\left(95 \% \mathrm{~N}_{2}-5 \% \mathrm{O}_{2}\right) \\
\mathrm{E}[\mathrm{J} / \mathrm{mol}] \\
\mathrm{A}\left[\mathrm{s}^{-1}\right]\end{array}$ \\
\hline 147.5 & $\begin{array}{l}n=1.5 \\
E=64218 \\
A=54\end{array}$ & 158 & $\begin{array}{l}n=1.5 \\
E=69749 \\
A=43\end{array}$ \\
\hline 100.8 & $\begin{array}{l}n=2 \\
E=77225 \\
A=245\end{array}$ & 101 & $\begin{array}{l}n=2 \\
E=92204 \\
A=1261\end{array}$ \\
\hline 49.7 & $\begin{array}{l}n=2 \\
E=82146 \\
A=313\end{array}$ & $(*)$ & \\
\hline
\end{tabular}




\begin{tabular}{|c|c|c|c|}
\hline $\begin{array}{c}\text { Taux de } \\
\text { chauffage } \\
{\left[{ }^{\circ} \mathrm{C} / \mathrm{min}\right]}\end{array}$ & $\begin{array}{c}\text { Milieu neutre } \\
\left(100 \% \mathrm{~N}_{2}\right) \\
\mathrm{E}[\mathrm{J} / \mathrm{mol}] \\
\mathrm{A}\left[\mathrm{s}^{-1}\right]\end{array}$ & $\begin{array}{c}\text { Taux de } \\
\text { chauffage } \\
{\left[{ }^{\circ} \mathrm{C} / \mathrm{min}\right]}\end{array}$ & $\begin{array}{c}\text { Milieu orydant } \\
\left(95 \% \mathrm{~N}_{2}-5 \% \mathrm{O}_{2}\right) \\
\mathrm{E}[\mathrm{J} / \mathrm{mol}] \\
\mathrm{A}\left[\mathrm{s}^{-1}\right]\end{array}$ \\
\hline 150.6 & $\begin{array}{c}\mathrm{n}=1.5 \\
\mathrm{E}=58518 \\
\mathrm{~A}=23\end{array}$ & 152.9 & $\begin{array}{l}\mathrm{n}=1.5 \\
\mathrm{E}=75834 \\
\mathrm{~A}=108\end{array}$ \\
\hline 97.2 & $\begin{array}{l}\mathrm{n}=2 \\
\mathrm{E}=84764 \\
\mathrm{~A}=768\end{array}$ & 101.0 & $\begin{array}{l}\mathrm{n}=2 \\
\mathrm{E}=74080 \\
\mathrm{~A}=110\end{array}$ \\
\hline 48.0 & $\mathrm{n}=2$ & & \\
\hline & $\mathrm{E}=77856$ & $(*)$ & \\
\hline
\end{tabular}

\begin{tabular}{|c|c|c|c|}
\hline $\begin{array}{c}\text { (d) } \\
\text { Taux de } \\
\text { chauffage } \\
{\left[{ }^{\circ} \mathrm{C} / \mathrm{min}\right]}\end{array}$ & $\begin{array}{c}\text { Milieu neutre } \\
\left(100 \% \mathrm{~N}_{2}\right) \\
\mathrm{E}\left[\mathrm{J} / \mathrm{mol}^{-1}\right] \\
\mathrm{A}\left[\mathrm{s}^{-1}\right]\end{array}$ & $\begin{array}{c}\text { Taux de } \\
\text { chauffage } \\
{\left[{ }^{\circ} \mathrm{C} / \mathrm{min}\right]}\end{array}$ & $\begin{array}{c}\text { Milieu oxydant } \\
\left(95 \% \mathrm{~N}_{2}-5 \% \mathrm{O}_{2}\right) \\
\mathrm{E}[\mathrm{J} / \mathrm{mol}] \\
\mathrm{A}\left[\mathrm{s}^{-1}\right]\end{array}$ \\
\hline 147.2 & $\begin{array}{c}\mathrm{n}=1.5 \\
\mathrm{E}=65135 \\
\mathrm{~A}=50\end{array}$ & 156.7 & $\begin{array}{c}\mathrm{n}=1.5 \\
\mathrm{E}=82778 \\
\mathrm{~A}=156\end{array}$ \\
\hline 97.2 & $\begin{array}{l}\mathrm{n}=2 \\
\mathrm{E}=82083 \\
\mathrm{~A}=336\end{array}$ & $(* *)$ & \\
\hline 47.8 & $\mathrm{n}=2$ \\
& $\mathrm{E}=96443$ \\
$\mathrm{~A}=1730$ & $(* *)$ & \\
\hline
\end{tabular}

(**) : Combustion du coke CONOCO à partir de $100^{\circ} \mathrm{C} / \mathrm{min}$. 


\section{VII.3 Modèle cinétique global de combustion du résidu de coke}

L'expression générale de la vitesse de combustion d'un solide s'exprime par la relation générale suivante $[9,11,15,20,22]$ :

$$
\mathrm{q}=\mathrm{k}(\mathrm{pg})^{\mathrm{n}}
$$

où $\mathrm{q}$ est la vitesse de combustion du solide en $\mathrm{g} / \mathrm{s}$, pg est la pression partielle en oxygène dans le milieu en atm et $\mathrm{k}$ la constante cinétique en g/s.atm ${ }^{\mathrm{n}}$.

La détermination de la constante cinétique $\mathrm{k}$ à chaque température repère à partir de l'équation (VII.4), nécessite de faire varier la pression partielle en oxygène, dans les conditions où le coke brûle par combustion, c'est-à-dire à $50^{\circ} \mathrm{C} / \mathrm{min}$ au cours de la calcination. En appliquant la relation d'Arrhénius à l'évolution de la constante $k$ avec la température, on détermine graphiquement l'énergie $\mathrm{E}$, l'ordre $\mathrm{n}$ et le facteur préexponentiel A. Or, cette procédure ne peut être envisagée dans le cadre de cette étude. En effet, lorsque le pourcentage d'oxygène dans le milieu dépasse $5 \%$ et que la vitesse de chauffage est lente, le déroulement de l'expérience est perturbé par la détérioration du creuset qui commence à brûler; si bien que les données relatives à la consommation du coke sous des pressions croissantes en oxygène n'ont pu être déterminées.

Néanmoins, une bonne approximation des paramètres cinétiques relatifs à la combustion du coke dans un milieu à $5 \%$ d'oxygène est obtenue à partir de l'équation générale (VII.1) appliquée à la variation du poids de coke par combustion à $50^{\circ} \mathrm{C} / \mathrm{min}$. Les figures 7.3 à 7.6 comparent les résultats expérimentaux et les résultats issus du modèle cinétique (VII.1), pour les quatre types de coke respectivement.

L'ordre de la réaction est fixé à 2; l'énergie d'activation fluctue entre 108 et $162[\mathrm{~kJ} / \mathrm{mol}$ ] pour la combustion des quatre cokes entre 750 et $1200^{\circ} \mathrm{C}$. Ces valeurs d'énergie d'activation se comparent bien avec celles précédemment observées par Smith, dans ses études sur la combustion des cokes, et notamment le coke de pétrole, en utilisant un modèle de type 
(VII.4) $[8,15]$. Les valeurs observées par Goetz, dans son étude sur les charbons, sont comprises entre 83 et $85 \cdot[\mathrm{kJ} / \mathrm{mol}]$ [13]; Hargrave par contre observe une énergie plus élevée $(95 \mathrm{~kJ} / \mathrm{mol})$ pour le coke de pétrole à haute température $\left(1200-2000^{\circ} \mathrm{C}\right)$ [9].

Il est normal de constater une légère fluctuation dans les énergies relatées par les différents auteurs, à cause des différentes techniques utilisées mais également de la large variété de charbons sur lesquels les études ont èté menées ainsi que les différentes conditions opérationnelles des expériences (taux de chauffage, gaz utilisés, granulométrie et taille des échantillons, etc.).

On remarque dans le tableau 7.2 que la combustion du coke Conoco à partir de $100^{\circ} \mathrm{C} / \mathrm{min}$ révèle une énergie d'activation fortement accrue $(365 \mathrm{~kJ} / \mathrm{mole})$, par rapport à $50^{\circ} \mathrm{C} / \mathrm{min}$. La figure 7.7 compare la combustion du coke CONOCO aux deux taux de chauffage 50 et $100^{\circ} \mathrm{C} / \mathrm{min}$.

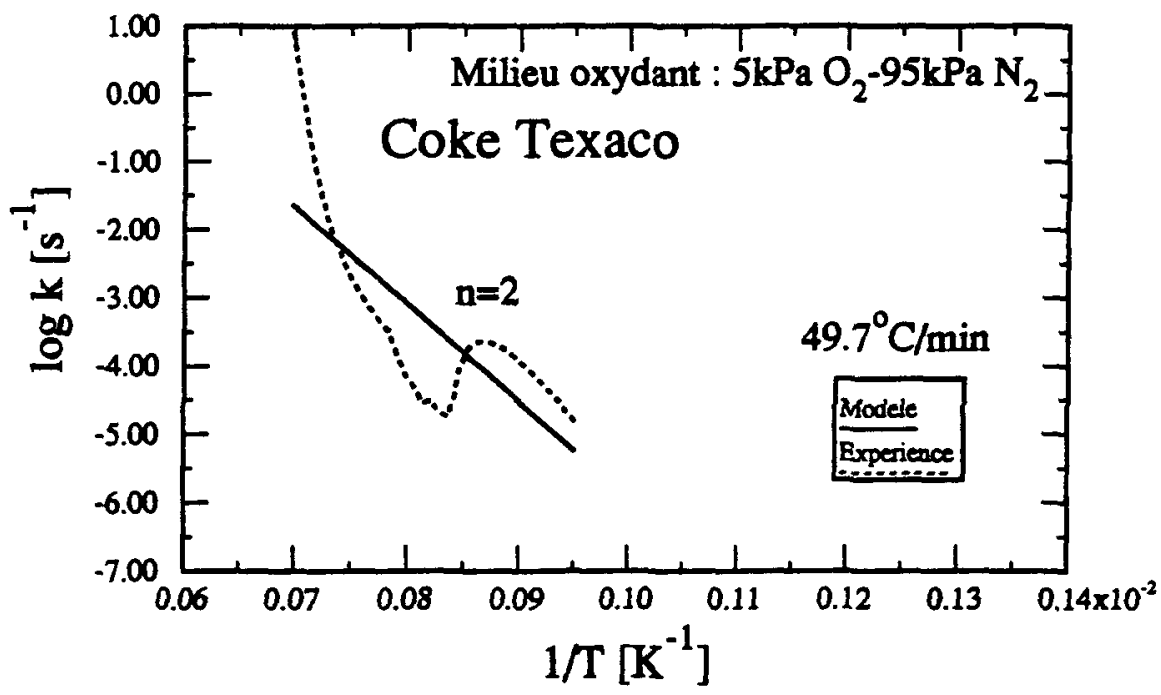

Figure 7.3: Cinétique de combustion du coke TEXACO à $50^{\circ} \mathrm{C} / \mathrm{min}$, en milieu oxydant ( $95 \%$ azote, $5 \%$ oxygène). 


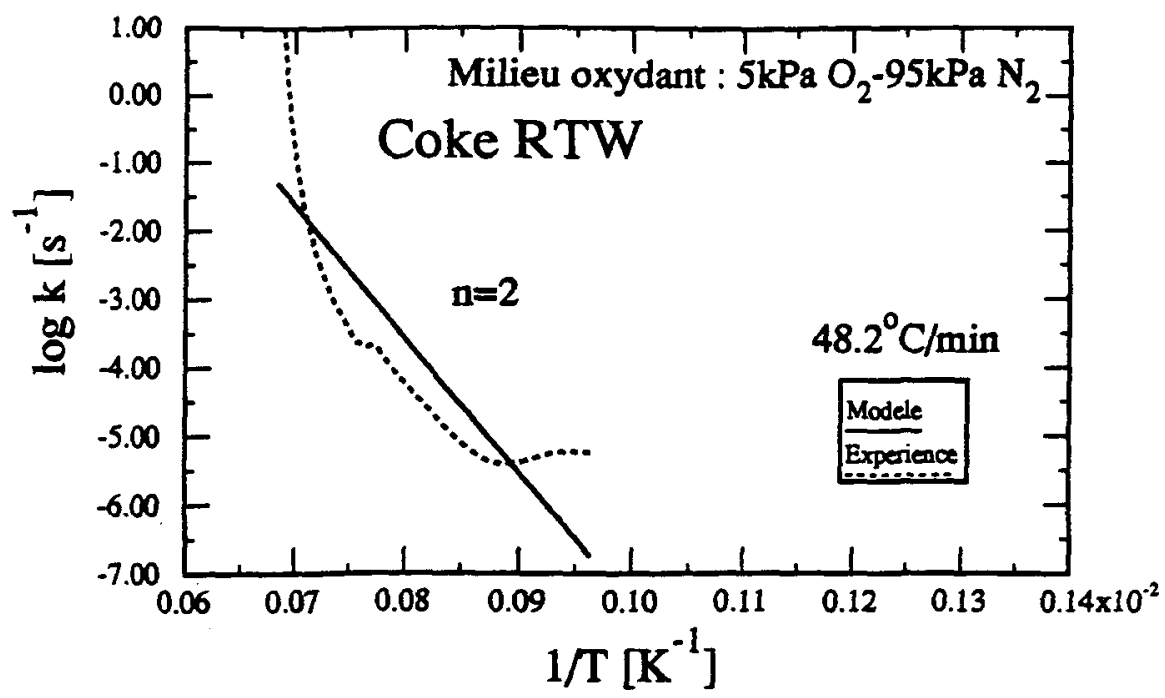

Figure 7.4: Cinétique de combustion du coke RTW à $50^{\circ} \mathrm{C} / \mathrm{min}$, en milieu oxydant ( $95 \%$ azote, $5 \%$ oxygène).

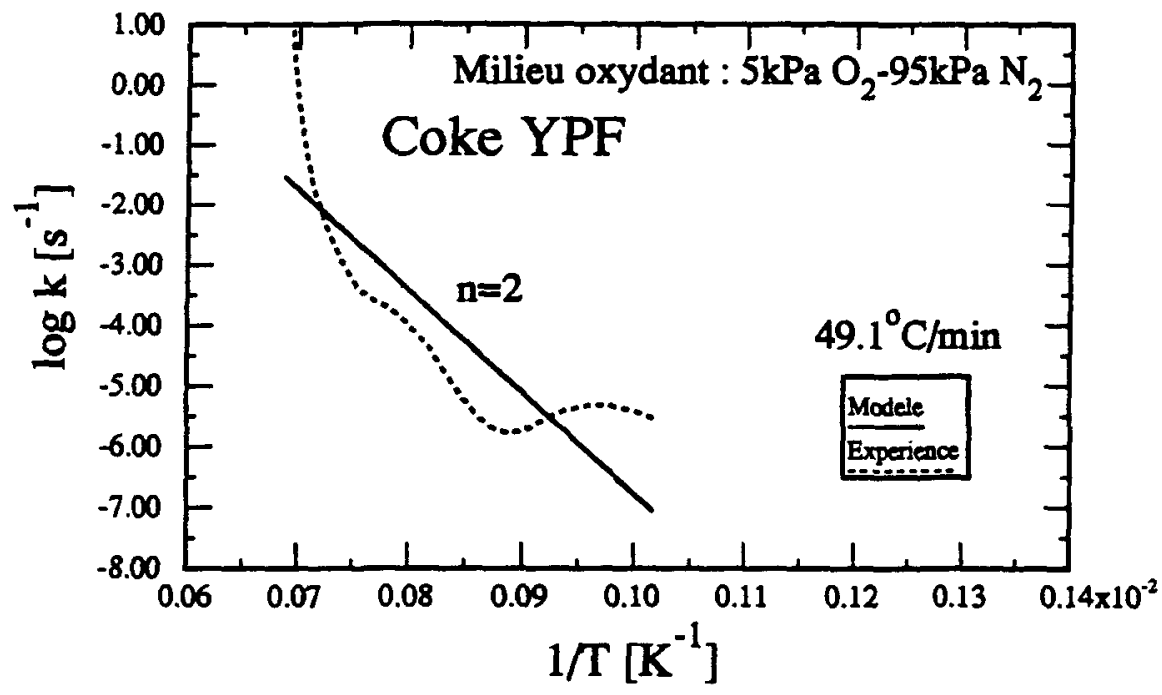

Figure 7.5: Cinétique de combustion du coke YPF à. $50^{\circ} \mathrm{C} / \mathrm{min}$, en milieu oxydant (95\% azote, $5 \%$ oxygène). 


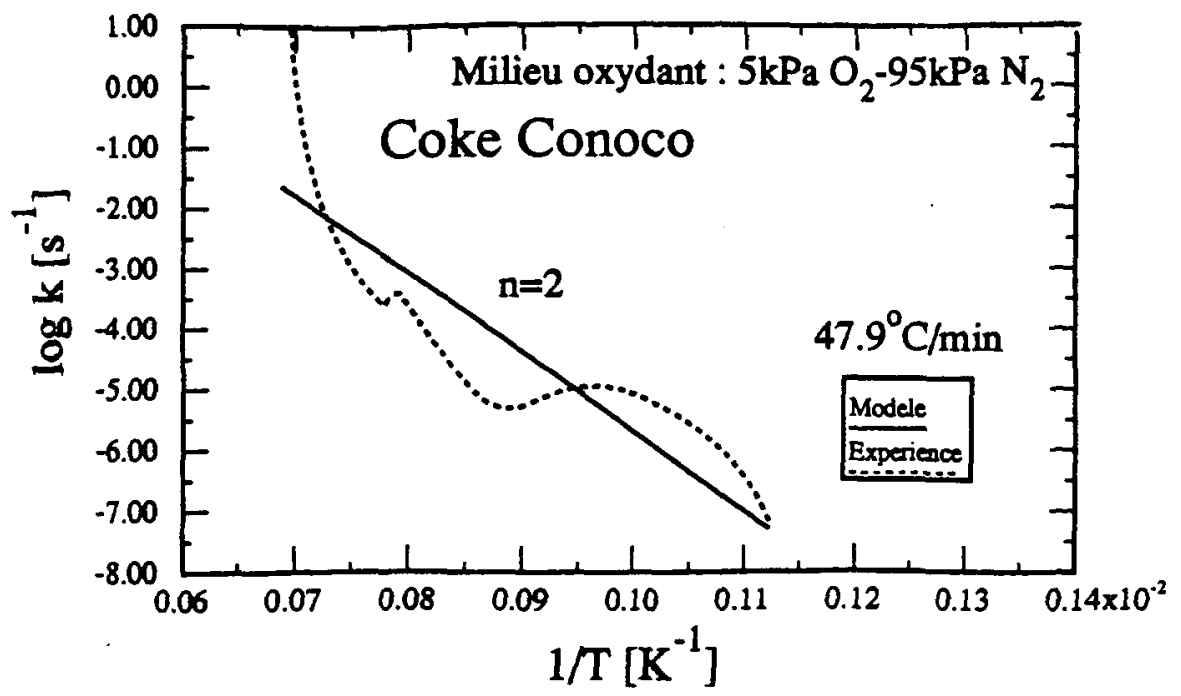

Figure 7.6: Cinétique de combustion du coke CONOCO à $50^{\circ} \mathrm{C} / \mathrm{min}$, en milieu oxydant (95\% azote, $5 \%$ oxygène).

Tableau 7.2: Paramètres cinétiques globaux de combustion du coke en milieu oxydant.

\begin{tabular}{|c|c|c|}
\hline $\begin{array}{c}\text { Taux de } \\
\text { chauffage } \\
\left.{ }^{\circ} \mathrm{C} / \mathrm{min}\right]\end{array}$ & Cokes & $\begin{array}{c}\text { Milieu oxydant } \\
\left(95 \% \mathrm{~N}_{2}-5 \% \mathrm{O}_{2}\right) \\
\mathrm{E} / \mathrm{J} / \mathrm{mol}] \\
\mathrm{A}\left[\mathrm{s}^{-1}\right]\end{array}$ \\
\hline 49.7 & TEXACO & $\begin{array}{l}\mathrm{n}=2 \\
\mathrm{E}=119007 \\
\mathrm{~A}=4243\end{array}$ \\
\hline 48.2 & RTW & $\begin{array}{l}\mathrm{n}=2 \\
\mathrm{E}=162023 \\
\mathrm{~A}=164679\end{array}$ \\
\hline 49.1 & YPF & $\begin{array}{l}\mathrm{n}=2 \\
\mathrm{E}=139952 \\
\mathrm{~A}=22987\end{array}$ \\
\hline 47.9 & CONOCO & $\begin{array}{l}\mathrm{n}=2 \\
\mathrm{E}=108857 \\
\mathrm{~A}=82\end{array}$ \\
\hline 96.6 & CONOCO & $\begin{array}{l}\mathrm{n}=2 \\
\mathrm{E}=365239 \\
\mathrm{~A}=2.410^{6}\end{array}$ \\
\hline & & $\mathrm{n}$ \\
\hline
\end{tabular}




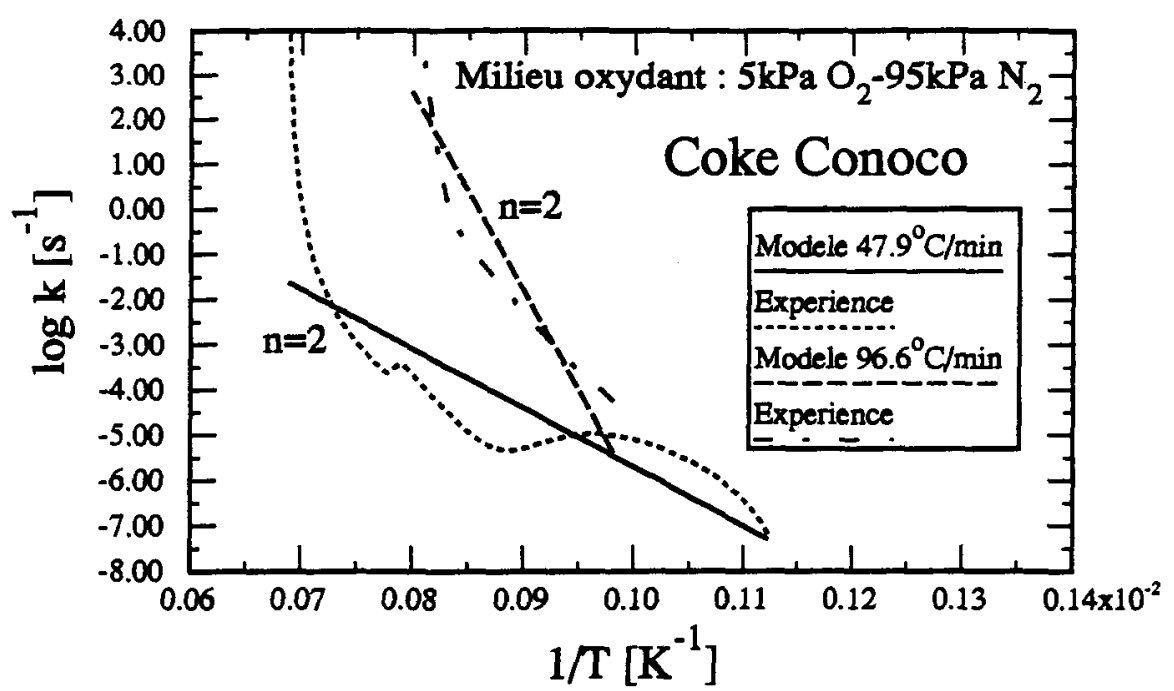

Figure 7.7 : Effet du taux de chauffage sur la combustion du coke CONOCO, en milieu oxydant ( $95 \%$ azote, $5 \%$ oxygène)

\section{VII.4 Conclusions}

Un modèle global basé sur la thermogravimétrie non isotherme [19], met en évidence l'influence de la nature du milieu réactionnel sur les paramètres cinétiques de dévolatilisation. La présence d'air dans le milieu réactionnel accroît l'énergie d'activation. L'ordre de la réaction est fixé à 2 , à haut taux de chauffage ( 100 et $\left.150^{\circ} \mathrm{C} / \mathrm{min}\right)$, et à 1.5 à $50^{\circ} \mathrm{C} / \mathrm{min}$.

De plus, le modèle global (VII.1) donne également une bonne idée de la cinétique de combustion du résidu de coke au cours de la calcination entre 750 et $1200^{\circ} \mathrm{C}$. L'énergie d'activation moyenne pour la combustion du coke de pétrole (moyenne sur les quatre cokes étudiés) entre $750^{\circ} \mathrm{C}$ et $1200^{\circ} \mathrm{C}$, au cours de la calcination à $50^{\circ} \mathrm{C} / \mathrm{min}$, vaut $132[\mathrm{~kJ} / \mathrm{mol}]$ pour un ordre de réaction global égal à 2 . L'énergie d'activation relative à la combustion du coke au cours de la calcination, augmente avec le taux de chauffage. 


\section{CHAPITRE VIII}

\section{ANALYSE STRUCTURALE}

\section{VIII.1 Introduction}

Au cours de la calcination du coke de pétrole, ce dernier subit de nombreuses modifications structurales concernant notamment la densité, la porosité, l'épaisseur cristalline et la résistivité électrique $[23,25,26]$. En effet, le dêpart des matières volatiles engendre un accroissement de la porosité mais également de la densité suite au grossissement et au réarrangement des cristaux au cours de la montée en température [12]. Les conditions de calcination (température, vitesse de chauffage, palier de température et nature du milieu) influencent la structure interne du coke et définissent la qualité des futures électrodes. La vitesse de chauffage est le facteur prédominant définissant le degré de développement des pores, luimême directement lié au pourcentage de matières volatiles [23].

Différentes analyses structurales des échantillons de coke prélevés au cours des expériences ont été effectuées. Etant donné le trop grand nombre d'échantillons recueillis, certaines analyses seulement ont été sélectionnées afin de mettre en évidence l'influence des conditions de travail sur la structure interne du coke calciné. On étudiera notamment l'effet de la température de calcination, du taux de chauffage, du palier de température et de la nature du milieu sur les propriêtés du coke calciné.

L'épaisseur cristalline est mesurée par diffraction des rayons $\mathrm{X}$ à l'aide d'un générateur Philips PW-1710 avec tube de cobalt. La densité réelle est mesurée à l'aide d'un picnomètre Régneault au kérosène. Les porosité et densité apparentes sont mesurées à l'aide du porosimètre au mercure. Des corrélations sont établies entre les conditions de calcination et les propriétés du coke calciné; les paramètres se trouvent dans le tableau 8.1. 


\section{VIII.2 Quelques définitions}

La densité réelle $D_{r}$ est définie comme étant le rapport entre la masse de solide et le volume de solide; alors que la densité apparente $D_{a}$ est donnée par la formule suivante:

$$
D_{a}=M_{s} /\left(V_{s}+V_{f}\right)
$$

où $M_{S}$ est la masse de solide, $V_{S}$ le volume de solide et $V_{f}$ le volume des pores fermés.

La porosité apparente $\mathrm{Pa}$ est définie par la formule suivante:

$$
P_{a}=V_{o} /\left(V_{s}+V_{f}+V_{o}\right)
$$

où $V_{0}$ est le volume des pores ouverts.

La porosité apparente est exprimée en \% et donnée pour des pores ouverts de diamètre supérieur à $64 \AA$. En effet, la pression dans le porosimètre est fixée à $2250 \mathrm{~atm}(33000 \mathrm{psi})$ de telle sorte que le mercure envahisse tous les pores ouverts de diamètre supérieur à $64 \AA$. Les pores ouverts de diamètre inférieur à $64 \AA$ sont considérés comme fermés ou non accessibles.

\section{VIII.3 Effet de la température de calcination}

\section{VIII.3.1 Sur l'épaisseur cristalline}

La figure 8.1 présente l'effet de la température de calcination sur la longueur cristalline du coke RTW en atmosphères neutre et oxydante. On constate que l'épaisseur cristalline augmente avec la température de calcination à partir de $900^{\circ} \mathrm{C}$, et ce dans les deux milieux. Cet accroissement de l'épaisseur cristalline avec la température a été observé par plusieurs auteurs et notamment Rhedey $[1,2,12,23,25,26]$.

Toutefois, la présence d'oxygène dans le milieu réduit l'épaisseur cristalline, et ce de manière plus significative à haute température (à partir de $1200^{\circ} \mathrm{C}$ ). 
Cette constatation signifie que, bien que le coke RTW n'entre pas en combustion à $150^{\circ} \mathrm{C} / \mathrm{min}$ (Annexe IV), la présence d'oxygène dans le milieu réactionnel affecte la structure interne du coke, en l'occurrence l'épaisseur cristalline. Ainsi, il semblerait que c'est la façon dont les produits volatils sont libérés au cours de la calcination qui définit, entre autre, l'épaisseur cristalline du coke calciné.

Il est difficile toutefois d'expliquer pourquoi le milieu oxydant n'agit pas de la même manière sur la longueur cristalline et la densité réelle, en comparant les figures 8.1 et 8.3 .

\section{VIII.3.2 Sur la porosité apparente}

La figure 8.2 montre l'accroissement de la porositê apparente au cours de la calcination du coke RTW à $150^{\circ} \mathrm{C} / \mathrm{min}$, dans les milieux neutre et oxydant. Cette augmentation de la porosité en fonction de la température (et donc du degré de dévolatilisation), est due au départ des matières volatiles et est directement reliée au pourcentage de volatils contenu dans le coke [23]. Comme l'avait observé Rhedey dans son étude sur le coke de pétrole [23], la présence d'oxygène dans le milieu ne modifie pas significativement la porosité apparente.

\section{VIII.3.3 Sur les densités réelle et apparente}

Les figures 8.3 à 8.4 présentent l'effet de la température de calcination sur les densités réelle et apparente du coke $\mathrm{RTW}$, à $150^{\circ} \mathrm{C} / \mathrm{min}$. On constate que la densité réelle croît linéairement au cours du chauffage en milieu neutre comme en milieu oxydant, avec cependant une pente légèrement moins accentuée pour le milieu oxydant. Les valeurs numériques très proches des densités apparentes et réelles signifient que le volume des pores fermés est très faible; néanmoins ce dernier semble croître au cours de la calcination $\left(\mathrm{D}_{\mathrm{a}}<\mathrm{D}_{\mathrm{r}}\right.$ à partir de $\left.1100^{\circ} \mathrm{C}\right)$. 


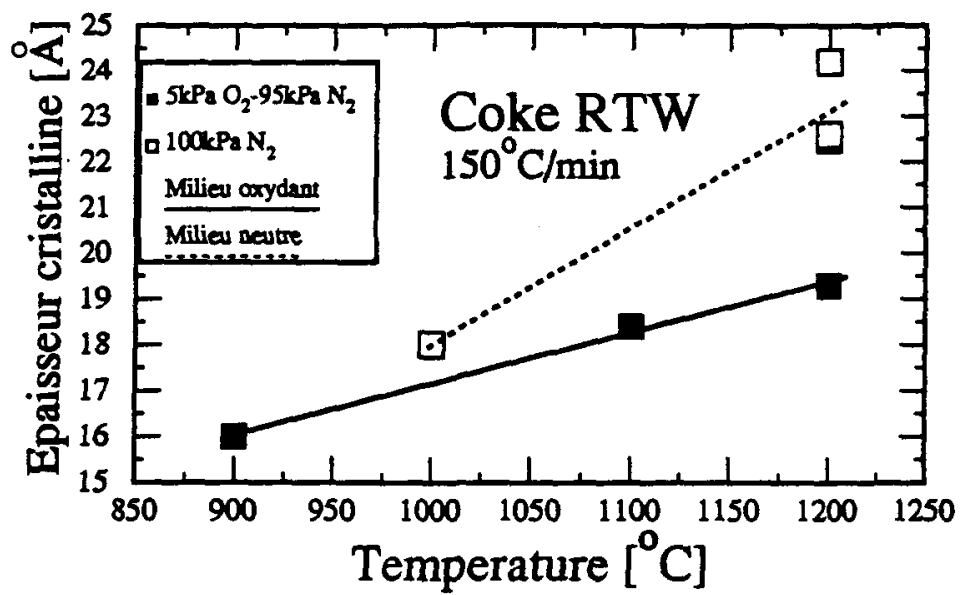

Figure 8.1 : Effet de la température de calcination sur l'êpaisseur cristalline.

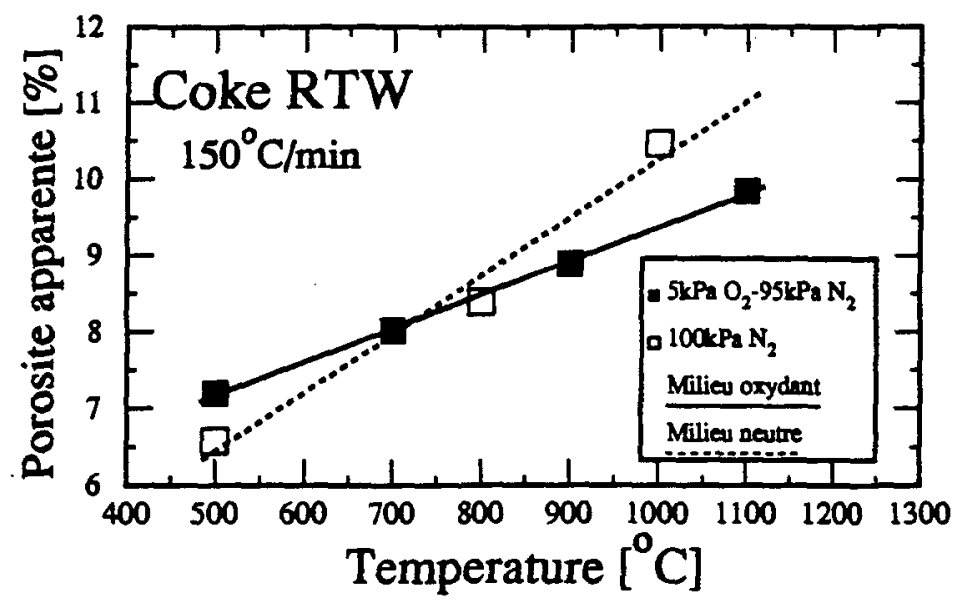

Figure 8.2 : Effet de la température de calcination sur la porosité apparente. 


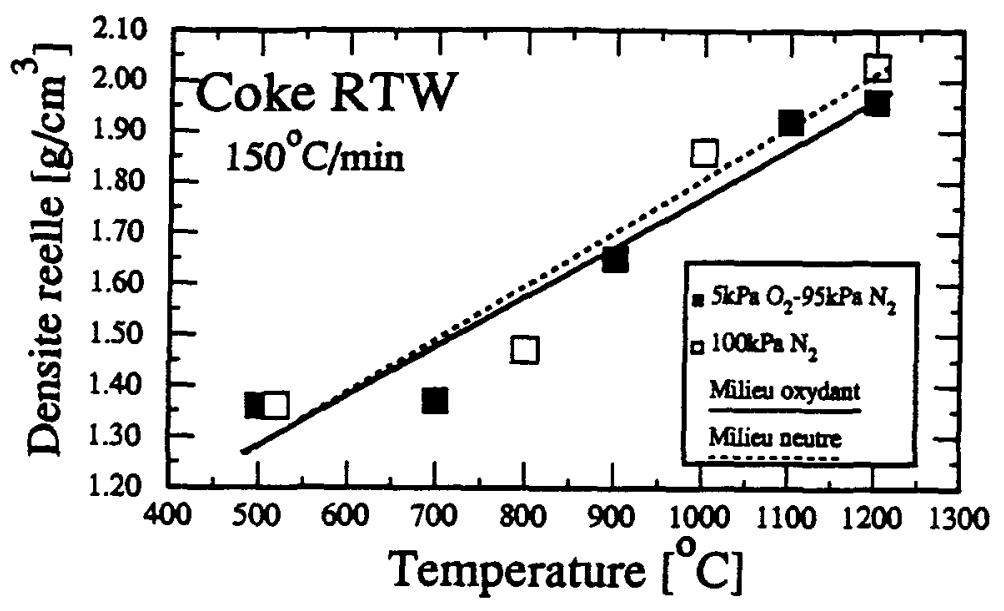

Figure 8.3 : Effet de la température de calcination sur la densité réelle.

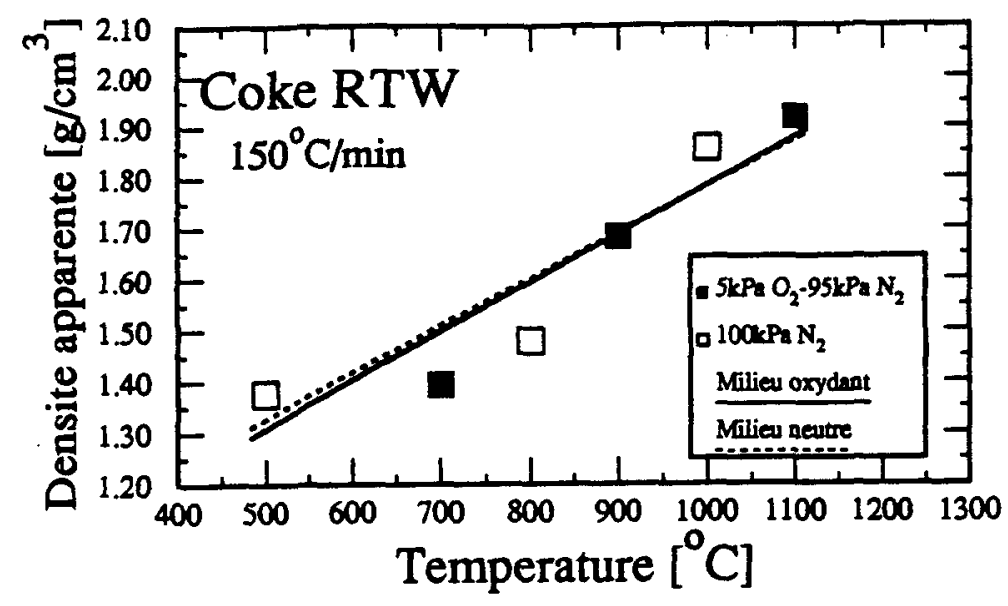

Figure 8.4: Effet de la température de calcination sur la densité apparente. 


\section{VIII.4 Effet du taux de chauffage}

\section{VIII.4.1 Sur l'épaisseur cristalline et la densité réelle}

Les figures 8.5 et 8.6 montrent l'effet du taux de chauffage sur l'épaisseur cristalline et la densité réelle du coke RTW récolté à $1200^{\circ} \mathrm{C}$, en milieux neutre et oxydant. On constate qu'un taux de chauffage faible augmente l'épaisseur cristalline ainsi que la densité réelle, surtout en atmosphère oxydante. En milieu neutre, l'épaisseur cristalline ne semble pas être très affectée par le taux de chauffage pour le coke RTW; néanmoins, une étude antérieure réalisêe sur d'autres cokes montrent la même tendance qu'en milieu oxydant [1]. On constate également que la présence d'oxygène diminue de manière plus significative l'épaisseur cristalline, à haut taux de chauffage $\left(150^{\circ} \mathrm{C} / \mathrm{min}\right)$.

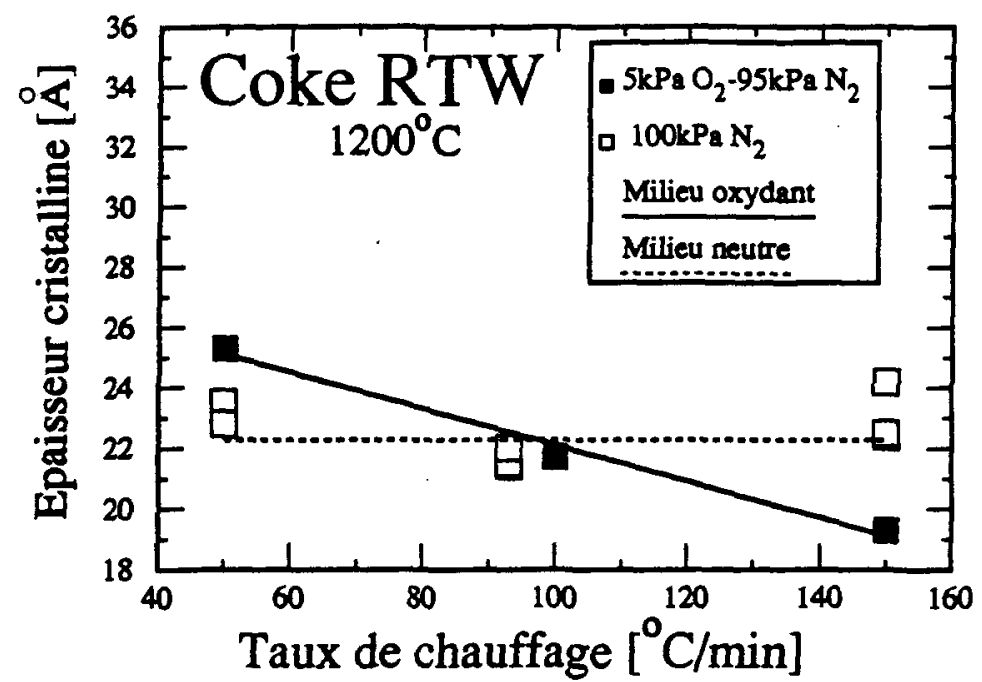

Figure 8.5 : Effet du taux de chauffage sur l'épaisseur cristalline. 


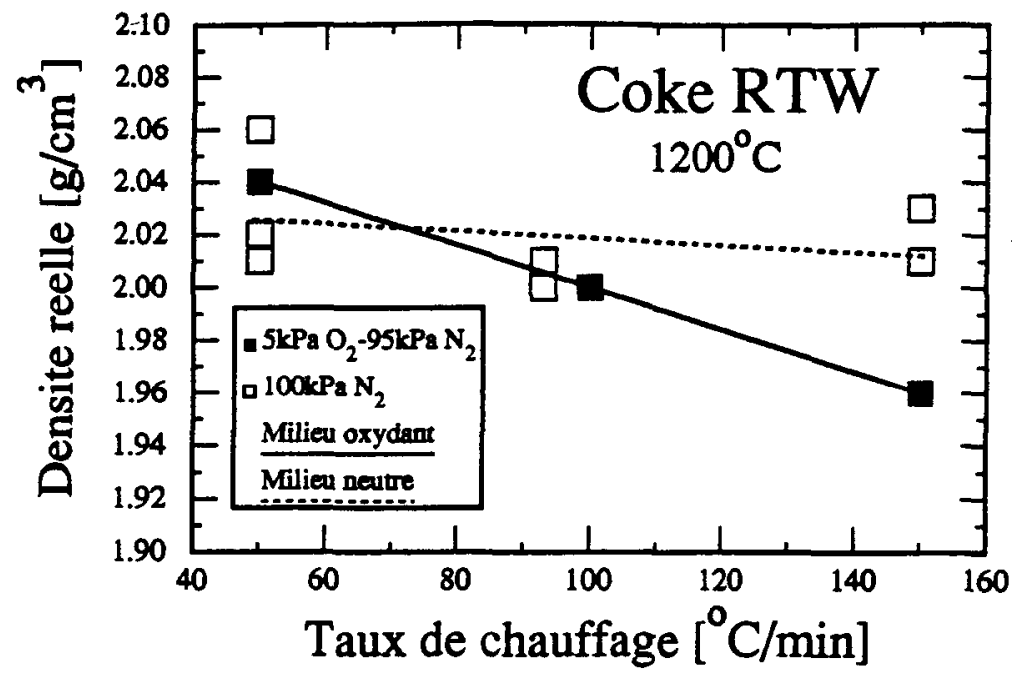

Figure 8.6 : Effet du taux de chauffage sur la densité réelle.

\section{VIII.5 Effet du palier de température à $1200^{\circ} \mathrm{C}$}

\section{VII.5.1 Sur l'épaisseur cristalline et la porosité apparente}

Les figures 8.7 et 8.8 montrent l'influence de la durée du palier à $1200^{\circ} \mathrm{C}$ sur l'épaisseur cristalline et la porosité apparente, pour le coke CONOCO. En milieu neutre, comme en milieu oxydant, on constate que l'épaisseur cristalline et la porosité apparente augmentent avec la durée du palier [1]. La différence entre les deux milieux devient de plus en plus marquante au fur et à mesure que la durée du palier de température à $1200^{\circ} \mathrm{C}$ augmente. La combustion du coke à haute température serait à l'origine de la modification de structure interne du coke, comme le stipule Rhedey dans une étude antérieure [23].

\section{VII.5.2 Sur les densités réelle et apparente}

Les figures 8.9 et 8.10 montrent l'évolution des densités réelle et apparente après un palier de 0,15 et 30 minutes à $1200^{\circ} \mathrm{C}$, pour le coke 
CONOCO chauffé à $150^{\circ} \mathrm{C} / \mathrm{min}$. On constate que l'accroissement de la densité suit la même évolution dans les deux milieux, avec cependant une valeur initiale à 0 minute plus élevée en milieu neutre par rapport au milieu oxydant.

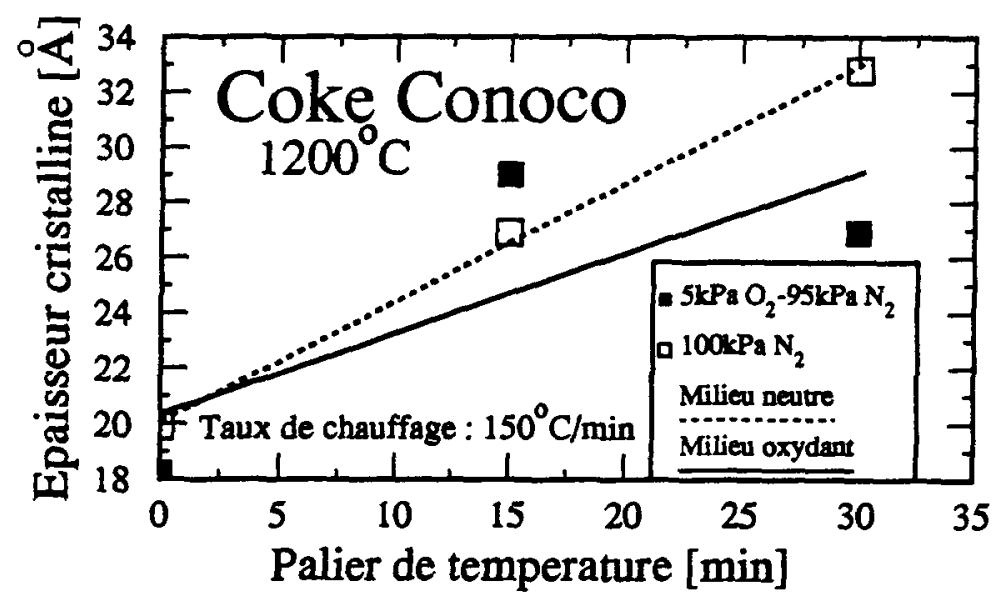

Figure 8.7 : Effet du palier de température sur l'épaisseur cristalline.

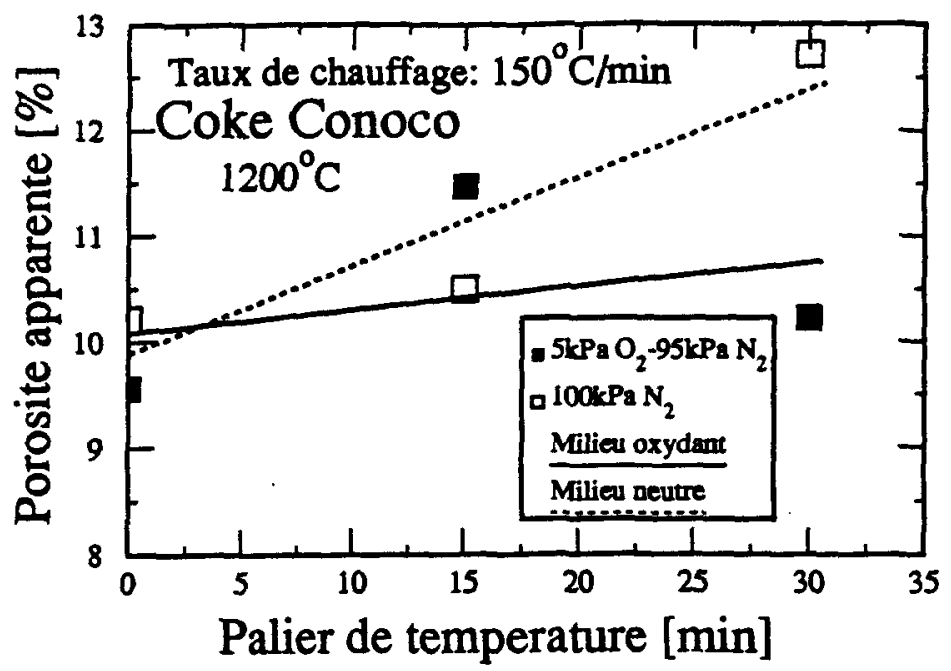

Figure 8.8 : Effet du palier de température sur la porosité apparente. 


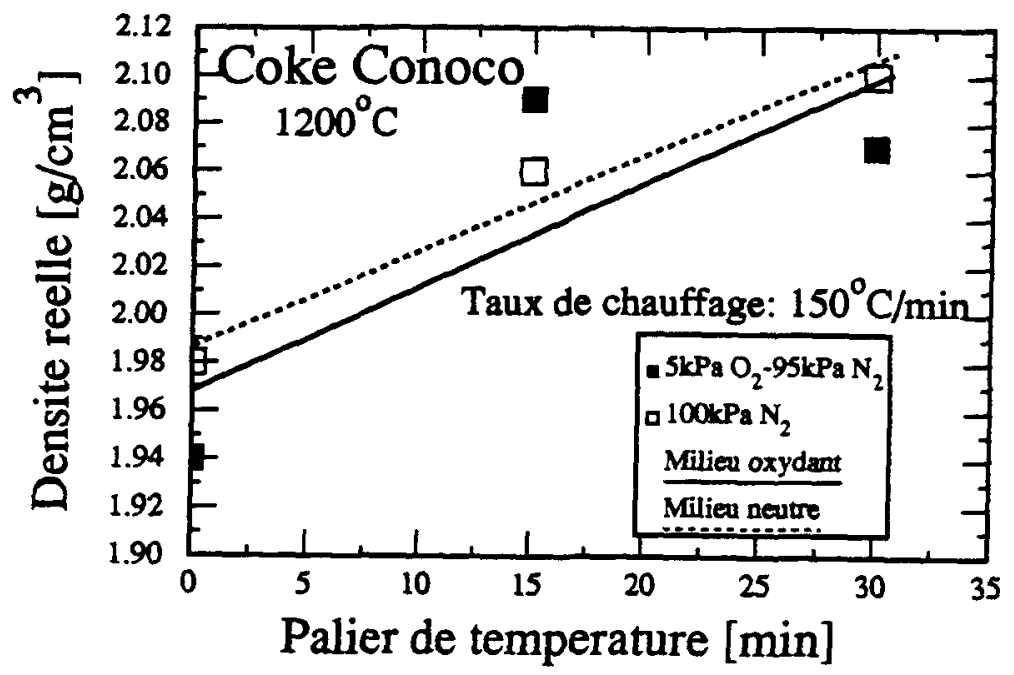

Figure 8.9 : Effet du palier de température sur la densité réelle.

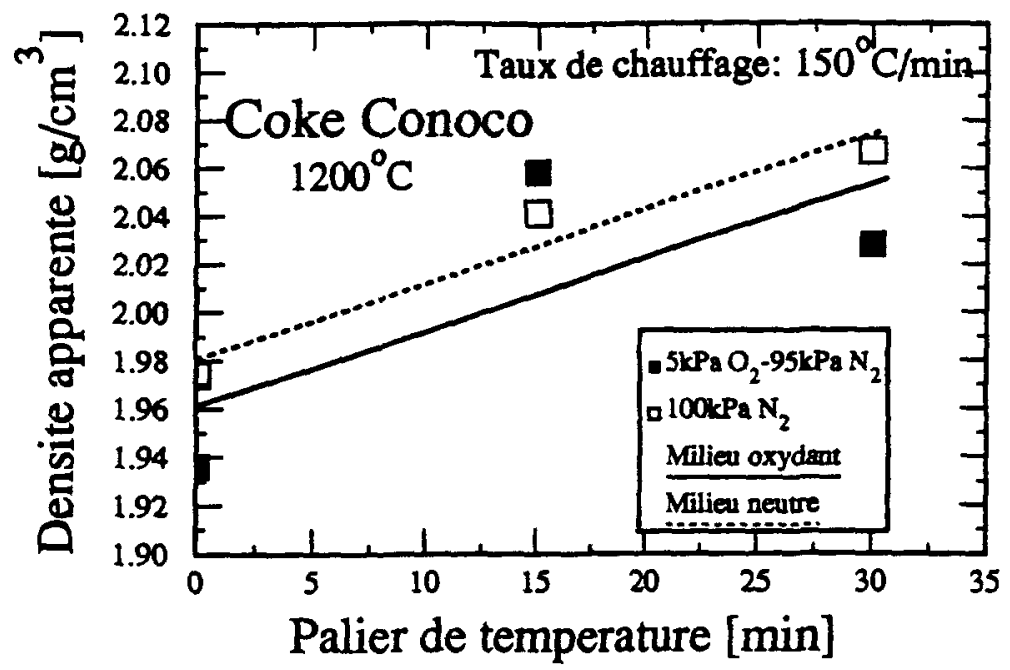

Figure 8.10 : Effet du palier de température sur la densité apparente. 


\section{VIII.6 Effet du pourcentage d'oxygène dans le milieu}

Les figures 8.11 et 8.12 montrent l'influence de la pression partielle en oxygène dans le milieu sur la longueur cristalline et la densité réelle, pour le coke TEXACO à $150^{\circ} \mathrm{C} / \mathrm{min}$. On peut constater qu'une faible proportion d'oxygène dans le milieu $(3 \mathrm{kPa})$ diminue considérablement la longueur cristalline ainsi que la densité réelle. L'oxygène en proportion plus élevée dans le milieu (jusqu'à $10 \mathrm{kPa}$ ) n'affecte pas davantage l'épaisseur cristalline et la densité réelle.

On constate, au regard de la figure 8.12 , que la densité réelle ne semble pas être très influencée par la combustion du coke débutant à partir de $7 \%$ d'oxygène dans le milieu (se référer à la figure 6.37). Cette constatation signifie que la combustion de la particule de coke se déroule bien par rétrécissement du diamètre de la particule plutôt que par pénétration de l'oxygène au sein de la structure poreuse du coke. Une analyse granulométrique des échantillons de coke prouve d'ailleurs que le diamètre moyen de la particule diminue au cours de la combustion. 


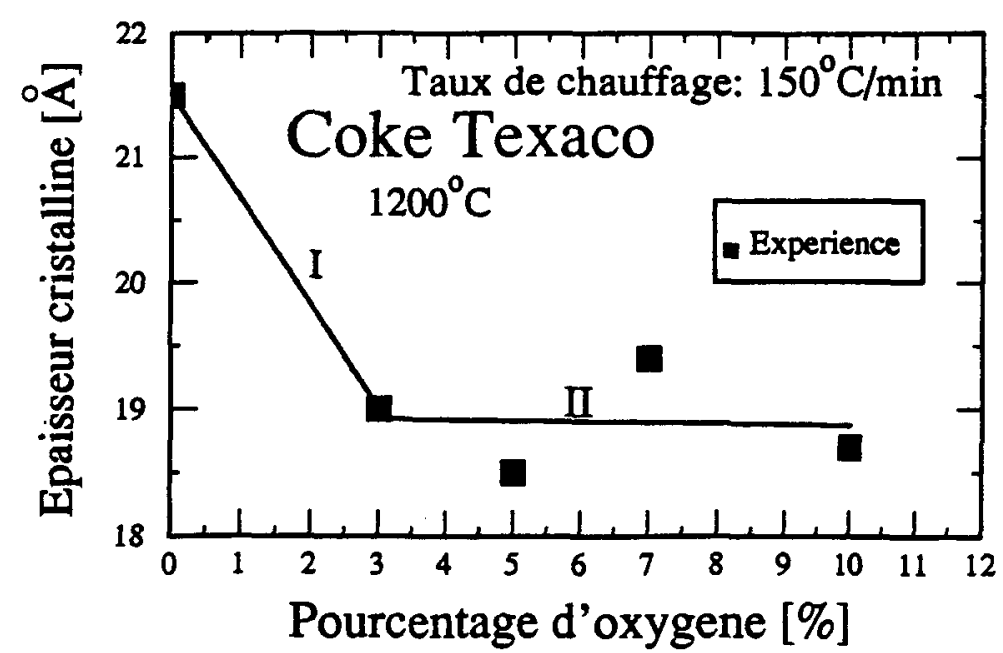

Figure 8.11 : Effet du pourcentage d'oxygène dans le milieu sur l'épaisseur cristalline.

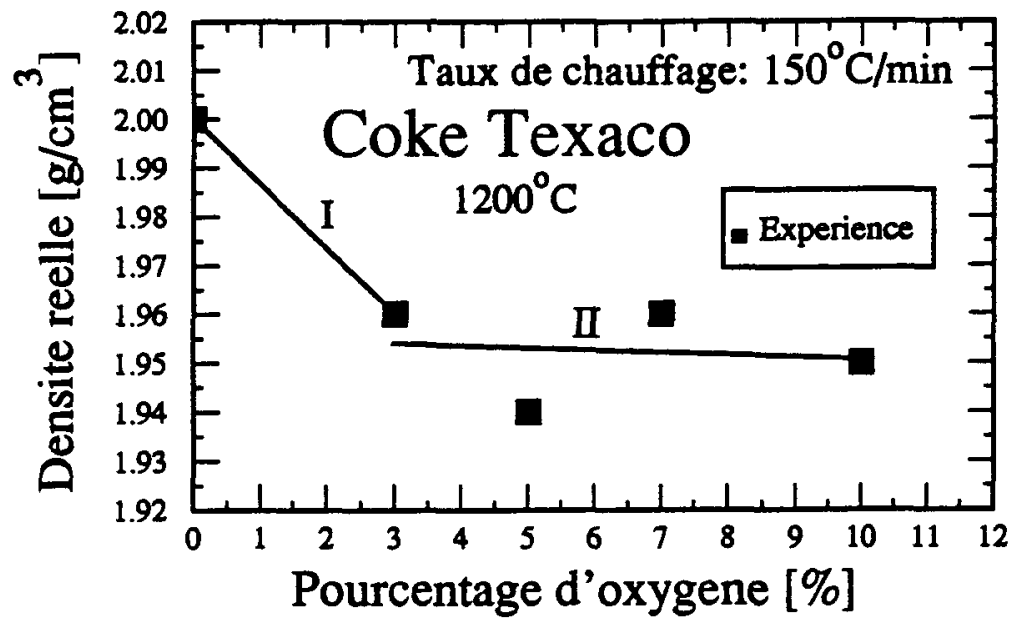

Figure 8.12 : Effet du pourcentage d'oxygène dans le milieu sur la densité réelle. 
Tableau 8.1: Paramètres des corrélations relatives aux propriétés structurales des cokes.

\begin{tabular}{|c|c|c|c|c|c|}
\hline \multirow[t]{2}{*}{ Figures } & \multirow[t]{2}{*}{$\mathbf{x}$} & \multirow[t]{2}{*}{$\mathbf{Y}$} & \multirow[t]{2}{*}{ Courbes } & \multicolumn{2}{|c|}{$\mathbf{Y}=\mathbf{A}_{0}+\mathbf{A}_{1} \mathbf{X}$} \\
\hline & & & & $\mathbf{A}_{0}$ & $\mathbf{A}_{1}$ \\
\hline \multirow[t]{2}{*}{ Fig 8.1} & \multirow[t]{2}{*}{$T$} & \multirow[t]{2}{*}{$L_{c}$} & Neutre & -7.500 & $2.55010^{-2}$ \\
\hline & & & Oxydant & 6.014 & $1.11010^{-2}$ \\
\hline \multirow[t]{2}{*}{ Fig 8.2} & \multirow[t]{2}{*}{$\mathrm{T}$} & \multirow[t]{2}{*}{$\mathrm{P}_{\mathbf{a}}$} & Neutre & 2.632 & $7.62310^{-3}$ \\
\hline & & & Oxydant & 4.971 & $4.39510^{-3}$ \\
\hline \multirow[t]{2}{*}{ Fig 8.3} & \multirow[t]{2}{*}{$\mathrm{T}$} & \multirow[t]{2}{*}{$D_{r}$} & Neutre & 0.755 & $1.05110^{-3}$ \\
\hline & & & Oxydant & 0.795 & $9.73110^{-4}$ \\
\hline \multirow[t]{2}{*}{ Fig 8.4} & \multirow[t]{2}{*}{$T$} & \multirow[t]{2}{*}{$D_{a}$} & Neutre & 0.872 & $9.10710^{-4}$ \\
\hline & & & Oxydant & 0.832 & $9.15010^{-4}$ \\
\hline \multirow[t]{2}{*}{ Fig 8.5} & \multirow[t]{2}{*}{ tc } & \multirow[t]{2}{*}{$\mathrm{L}_{c}$} & Neutre & 22.270 & 0.000 \\
\hline & & & Oxydant & 28.900 & -0.072 \\
\hline \multirow[t]{2}{*}{ Fig 8.6} & \multirow[t]{2}{*}{ tc } & \multirow[t]{2}{*}{$D_{r}$} & Neutre & 2.032 & $-1.33310^{-4}$ \\
\hline & & & Oxydant & 2.080 & $-8.00010^{-4}$ \\
\hline \multirow[t]{2}{*}{ Fig 8.7} & \multirow[t]{2}{*}{ pt } & \multirow[t]{2}{*}{$\mathrm{L}_{\mathrm{c}}$} & Neutre & 20.080 & 0.430 \\
\hline & & & Oxydant & 20.350 & 0.290 \\
\hline \multirow[t]{2}{*}{ Fig 8.8} & \multirow[t]{2}{*}{ pt } & \multirow{2}{*}{$\mathrm{P}_{\mathrm{a}}$} & Neutre & 9.886 & 0.083 \\
\hline & & & Oxydant & 10.083 & 0.022 \\
\hline \multirow[t]{2}{*}{ Fig 8.9} & \multirow[t]{2}{*}{ pt } & \multirow[t]{2}{*}{$D_{r}$} & Neutre & 1.968 & $4.33010^{-3}$ \\
\hline & & & Oxydant & 1.987 & $4.00010^{-3}$ \\
\hline \multirow[t]{2}{*}{ Fig 8.10} & pt & $D_{\mathbf{a}}$ & Neutre & 1.980 & $3.10110^{-3}$ \\
\hline & & & Oxydant & 1.961 & $3.06610^{-3}$ \\
\hline Fig 8.11 & $\mathrm{pO}_{2}$ & $\mathrm{~L}_{c}$ & I & 21.500 & -0.833 \\
\hline & & & II & 18.900 & $-7.40010^{-3}$ \\
\hline Fig 8.12 & $\mathrm{pO}_{2}$ & $D_{r}$ & I & 2.000 & $-1.33010^{-2}$ \\
\hline & & & II & 1.950 & $-4.67010^{-4}$ \\
\hline
\end{tabular}

T : Température $\left[{ }^{\circ} \mathrm{C}\right]$

tc : Taux de chauffage $\left[{ }^{\circ} \mathrm{C} / \mathrm{min}\right]$

pt : Palier de temperature à $1200^{\circ} \mathrm{C}$

$\mathrm{pO}_{2}$ : $\quad$ Pourcentage d'oxygène [\%]
$D_{\mathrm{r}}$ : Densité réelle $\left[\mathrm{g} / \mathrm{cm}^{3}\right]$

$\mathrm{D}_{\mathrm{a}}$ : Densité apparente $\left[\mathrm{g} / \mathrm{cm}^{3}\right]$

$\mathrm{Pa}_{\mathrm{a}}$ : Porosité apparente [\%]

$\mathrm{L}_{c} \quad$ : Epaisseur cristalline $[\AA]$ 


\section{VIII.6 Conclusions}

D'une manière générale, la présence de l'oxygène dans le milieu réactionnel, à raison de 5\% d'oxygène pour 95\% d'azote, tend à diminuer la valeur des paramètres structuraux comme l'épaisseur cristalline, la densité et la porosité, et ce à haut taux de chauffage $\left(150^{\circ} \mathrm{C} / \mathrm{min}\right)$, à haute température $\left(>1000^{\circ} \mathrm{C}\right)$ et après un palier à $1200^{\circ} \mathrm{C}$ supérieur à 15 minutes. L'accroissement du pourcentage d'oxygène dans le milieu jusqu'à $10 \%$ n'affecte pas davantage la structure du coke calciné.

Toutefois, on remarque les grandes tendances déjà observées antérieurement par d'autres auteurs $[1,12,21,23,25,26]$, à savoir:

- l'accroissement de l'épaisseur cristalline, des densités réelle et apparente et de la porosité apparente avec la température de calcination et la durée du palier à $1200^{\circ} \mathrm{C}$;

- le grossissement des cristaux et l'augmentation de la densité réelle avec la diminution de la vitesse de chauffage. 


\section{CHAPITRE DX}

\section{CONCLUSIONS GENERALES}

Le creuset mis au point dans le cadre de cette étude semble être bien adapté au contexte de la cinétique hétérogène solide-gaz, car il permet un contact aisé entre les particules solides et le gaz comburant, ainsi qu'un préchauffage des gaz dans la partie inférieure du creuset. Le milieu oxydant réduit significativement le gradient de température dans l'échantillon de coke vert suite aux réactions de combustion.

Contrairement à ce que certains auteurs prétendent, c'est uniquement à faible taux de chauffage $\left(50^{\circ} \mathrm{C} / \mathrm{min}\right)$ que la dévolatilisation du coke n'est pas influencée par la nature du milieu dans lequel la calcination est conduite. Lorsque le chauffage est plus rapide, la dévolatilisation est retardée en milieu oxydant, par rapport au milieu neutre. Un modèle cinétique compare les paramètres cinétiques globaux de dévolatilisation dans les deux milieux. Le milieu oxydant révèle une énergie d'activation supérieure à celle observée en milieu neutre, pour un ordre de réaction égal à $1.5\left(\right.$ à $150^{\circ} \mathrm{C} / \mathrm{min}$ ) et à 2 (à $100^{\circ} \mathrm{C} / \mathrm{min}$ ).

Dans certaines conditions de température, de vitesse de chauffage et de disponibilité en oxygène dans le milieu, le taux de consommation total du coke par combustion n'atteint que 1 à $2 \%$ à $1200^{\circ} \mathrm{C}$ (en fin de calcination). De ce fait, il importait davantage, dans le cadre de cette étude, de déterminer les conditions dans lesquelles la combustion du résidu de coke s'amorce, plutôt que de s'attacher à sa cinétique proprement dite de combustion. Etant donné les difficultés expérimentales rencontrées en atmosphère oxydante sous des vitesses de chauffe lentes et des pressions partielles élevées en oxygène (détérioration du creuset à partir de $50^{\circ} \mathrm{C} / \mathrm{min}$ et $10 \%$ d'oxygène, explosion à $800^{\circ} \mathrm{C}$ sous $10 \%$ d'oxygène et $150^{\circ} \mathrm{C} / \mathrm{min}$ ), un modèle global similaire à celui de la dévolatilisation a été utilisé pour caractériser la combustion du résidu carboné. Ce modèle révèle une énergie 
d'activation relativement proche de celle trouvée par d'autres auteurs (Smith, Hargrave et Goetz).

L'analyse structurale du coke calciné montre une densité plus ou moins constante du coke avec l'augmentation de la pression partielle en oxygène dans le milieu, ainsi qu'une diminution du diamètre des particules au cours de la combustion du coke. On peut donc affirmer que, dans nos conditions de travail, la combustion du résidu de coke au cours de la calcination est régie par les deux phénomènes limitants suivants: la diffusion interne dans la structure poreuse du coke et la réaction chimique intrinsèque (régime II).

En atmosphère oxydante $\left(5 \mathrm{kPa} \mathrm{O}_{2}-95 \mathrm{kPa} \mathrm{N} \mathrm{N}_{2}\right)$, et lorsque le taux de chauffage est supérieur ou égal à $100^{\circ} \mathrm{C} / \mathrm{min}$, le coke résiduel n'entre pas en combustion au cours du traitement de calcination. En fait, les matières volatiles, une fois dégagées de la masse solide, constituent une protection autour de la particule; cette protection se consume petit à petit avec l'oxygène jusqu'au moment où, devenue trop mince, l'oxygène comburant attaque la particule.

On constate que la combustion du coke est favorisée par:

- un taux de chauffage lent, à cause de la durée de l'expérience qui tient les volatils plus longtemps au contact de l'oxygène comburant;

- un indice faible en matières volatiles du coke, puisque ce sont ces volatils en quantité plus ou moins importante qui protègent le coke de la combustion;

- et enfin, un pourcentage d'oxygène élevé dans le milieu, puisque cette condition engendre une combustion plus rapide et plus complète des volatils de protection.

En ce qui concerne la combustion des volatils légers, on constate qu'un taux de chauffage lent et une disponibilité élevée en oxygène dans le milieu favorise la combustion des volatils de type hydrogène et méthane. La cinétique de combustion des produits volatils est bien réelle comme semble le prouver le calcul des concentrations à l'équilibre. L'intervalle de température pendant lequel la combustion en atmosphère oxydante a lieu correspond exactement à l'intervalle de dégagement de ces mêmes produits en l'absence d'air, et ce aux trois taux de chauffage. La combustion rapide des condensables lourds est visuellement perçue au cours de la calcination 
en atmosphère oxydante, par la présence d'une fumée blanche, chargée d'humidité dans les premiers stades du traitement thermique (entre 550 et $700^{\circ} \mathrm{C}$ ).

En ce qui concerne l'évolution structurale du coke au cours de la calcination, on constate que la présence de l'oxygène dans le milieu n'entrave pas les grandes tendances générales, déjà observées par bon nombre d'auteurs et qui sont:

- un accroissement de l'épaisseur cristalline avec la température de calcination (entre 900 et $1200^{\circ} \mathrm{C}$ ), la diminution du taux de chauffage et l'augmentation de la durée du palier de température à $1200^{\circ} \mathrm{C}$;

- une augmentation de la densité et de la porosité apparente avec la température (entre 500 et $1200^{\circ} \mathrm{C}$ ), la diminution du taux de chauffage et l'augmentation de la durée du palier de température à $1200^{\circ} \mathrm{C}$.

Néanmoins, il faut remarquer une légère diminution de l'épaisseur cristalline à $1200^{\circ} \mathrm{C}$ en milieu oxydant, par rapport au milieu neutre. Un chauffage lent favorise le grossissement des cristaux en milieu oxydant comme en milieu neutre, Lors du palier de température effectué à $1200^{\circ} \mathrm{C}$, la présence d'oxygène dans le milieu réduit la valeur des paramètres structuraux (épaisseur cristalline, densité et porosité) surtout à partir de 30 minutes de palier. 


\section{BIBLIOGRAPHIE}

[1] D. Kocaefe, A. Charette and L. Castonguay

"Investigation of structural changes during green coke

calcination", International Carbon Conference, pp 72-74, 1992

[2] A. Charette, D. Kocaefe

"Study of green coke calcination", Rapport technique No 34 , GRIPS (UQAC), 1992

[3] A Charette, D. Kocaefe, L. Castonguay

"Study of green coke calcination", pp 619-622,

Lights Metals, 1992

[4] T.F. Wall, V.S. Gururajan, J. Lucas, R.P. Gupta, Zhang Dong-Ke and I.W. Smith and B.C. Young

"The ignition, burning rate and reactivity of petroleum coke", Twenty-third Symposium on Combustion, The Combustion Institute, pp 1177-1184, 1990

[5] D. Kocaefe, A. Charette, J. Ferland, P. Couderc, J.L. St Romain "A kinetics study of pyrolysis in pitch impregnated electrodes", The Canadian Journal of Chemical Engineering, 68, pp 988-996, 1990

[6] F. Tremblay and A. Charette

"Cinétique de dégagement des matières volatiles lors de la pyrolyse d'électrodes de carbone industrielles", The Canadian Journal of Chemical Engineering, 66, pp 86-95, 1988

[7] P.F Nelson, I.W. Smith, R.J. Tyler "Pyrolysis of coal at high temperatures",

American Chemical Society, Division of fuel chemistry, pp 142-147, 1987

[8] W. Rybak, M. Zembrzuski and I.W. Smith

"Kinetics of combustion of petroleum coke and sub-bituminous coal chars: results of ignition and steady-state techniques", Twenty-first Symposium on Combustion, The Combustion Institue, pp 231-237, 1986

[9] G. Hargrave, M. Pourkashanian and A. Williams "The combustion and gasification of coke and coal chars", Twenty-first Symposium on Combustion, The Combustion Institute, pp 221-230, 1986 
[10] G. Borghi, A.F. Sarofim and J.M. Beér

"A model of coal devolatilization and combustion in fluidized beds", Combustion and Flame, 61, pp 1-16, 1985

[11] M.A. Andrei, A.F. Sarofim, J.M. Beér

"Time-resolved burnout of coal particles in a fluidized bed", Combustion and Flame, 61, pp 17-67, 1985

[12] W.K. Fisher and R.Perruchoud

"Influence of calcining parameters on petroleum coke quality", Lights Metals, pp 812-826, 1985

[13] G.J. Goetz, Nsakala Ya Nsakala and R.L. Patel

"Combustion and gasification kinetics of chars from four commercially significant coals of varying rank", Int. Conf. Coal Sci., pp 571-574, 1983

[14] I.W. Smith

"The combustion rates of coal chars: a review", Nineteenth Symposium on Combustion, The Combustion Institute, pp 1045-1065, 1982

[15] B.C. Young and I.W Smith

"The kinetics of combustion of petroleum coke particles at 1000 to 1800K: the reaction order", Eighteenth Symposium on Combustion, The Combustion Institute, pp 1249-1254, 1981

[16] E.M. Suuberg, W.A. Peters and J.B. Howard "Product compositions and formation kinetics in rapid pyrolysis of pulverised coal - implications for combustion", Seventeenth Symposium on Combustion, The Combustion Institute, pp 117-130, 1978

[17] J. Belleau

"La dévolatilisation du coke lors de sa calcination", Rapport technique, Alcan International Ltée, 1978

[18] D.B. Anthony, J.B. Howard, H.C. Hottel and H.P. Meissner "Rapid devolatilization of pulverised coal", Fifteenth Symposium on Combustion, The Combustion Institute, pp 1303-1317, 1975

[19] R.W. Wallouch, H.N. Murty, E.A. Heintz "Pyrolysis of coal tar pitch binders", Carbon, 10, pp 729-735, 1972 
[20] I.W. Smith

"Kinetics of combustion of size-graded pulverised fuels in the temperature range 1200-2270K",

Combustion and Flame, 17, pp 303-314, 1971

[21] S. L. Polak

"Petroleum coke: Volume and composition of volatiles evolved during calcination",

Rapport technique No A-RR-1475-71-08, Alcan

International Ltée, 1971

[22] M.A. Field, D.W. Gill, B.B. Morgan, P.G.W. Hawksley

"Combustion of pulverised coal", BCURA, 1967

[23] P. Rhedey

"Structural changes in petroleum coke during calcination", Transactions of the Metallurgical Society of AIME, 239, pp 1084-1091, 1967

[24] Groupe français d'étude des carbones

"Les carbones", Tomes I et II,

Collection de chimie-physique, Masson et Cie, 1965

[25] P. Rhedey

"Study of petroleum coke aggregate for carbon electrodes", Rapport technique No A-RR-970-64-19, Alcan

International Ltée, 1964

[26] P. Rhedey

"Study of petroleum coke aggregate for carbon electrodes", Rapport technique No A-RR-876-63-19, Alcan

International Ltée, 1963

[27] P.L. Walker, F. Rusinko and L. Austin

"Gas reactions of carbon, Advances in catalysis", 10, Academic Press Inc., pp 133-221, 1959 
Annexe I: $\quad$ Effet du taux de chauffage sur l'hydrogène et le méthane recueillis en milieu neutre ( $100 \%$ azote), pour les trois autres cokes (RTW, YPF et CONOCO). 


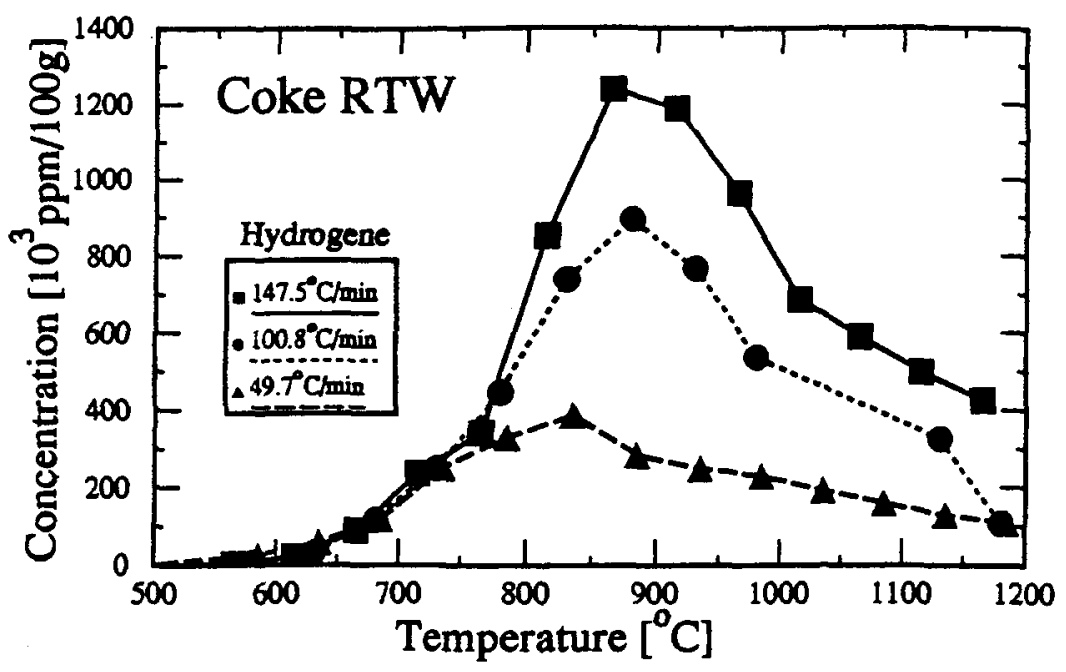

Annexel.1 : Effet du taux de chauffage sur l'hydrogène du coke RTW, en milleu neutre $(100 \%$ azote).

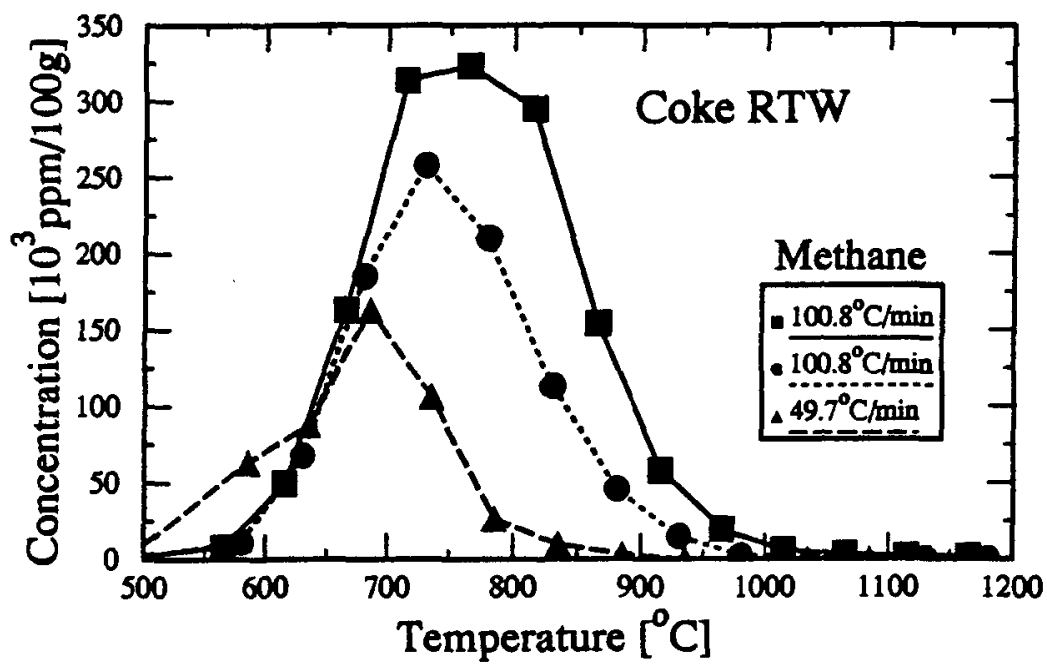

Annexel.2 : Effet du taux de chauffage sur le méthane du coke RTW, en milleu neutre (100\% azote). 


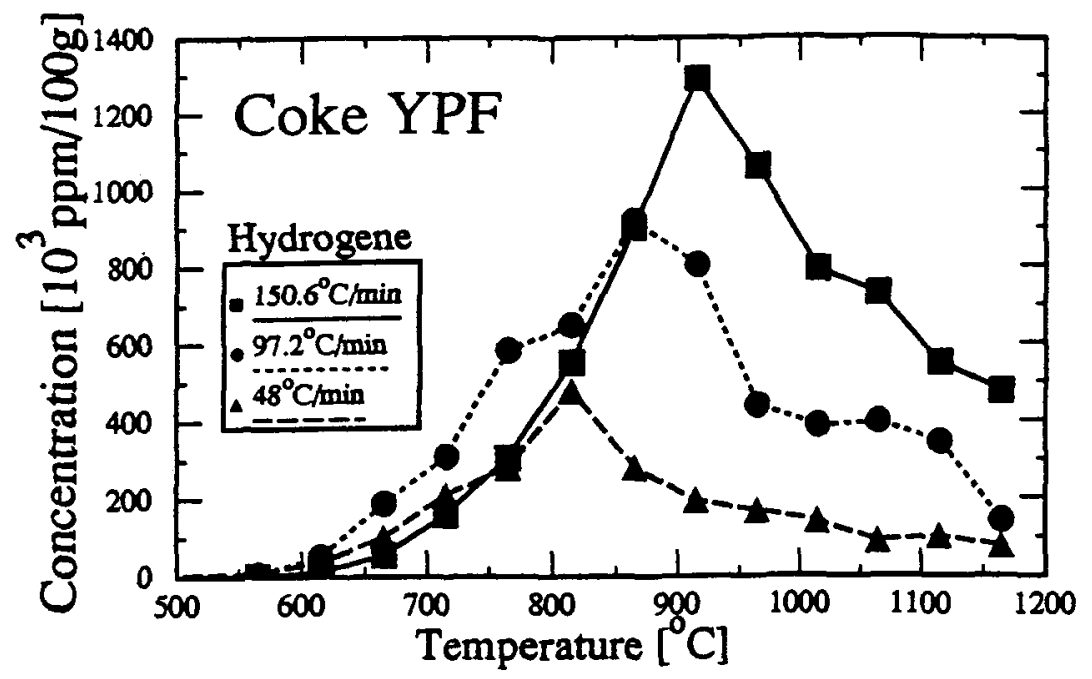

Annexel.3 : Effet du taux de chauffage sur l'hydrogène du coke YPF, en milieu neutre ( $100 \%$ azote).

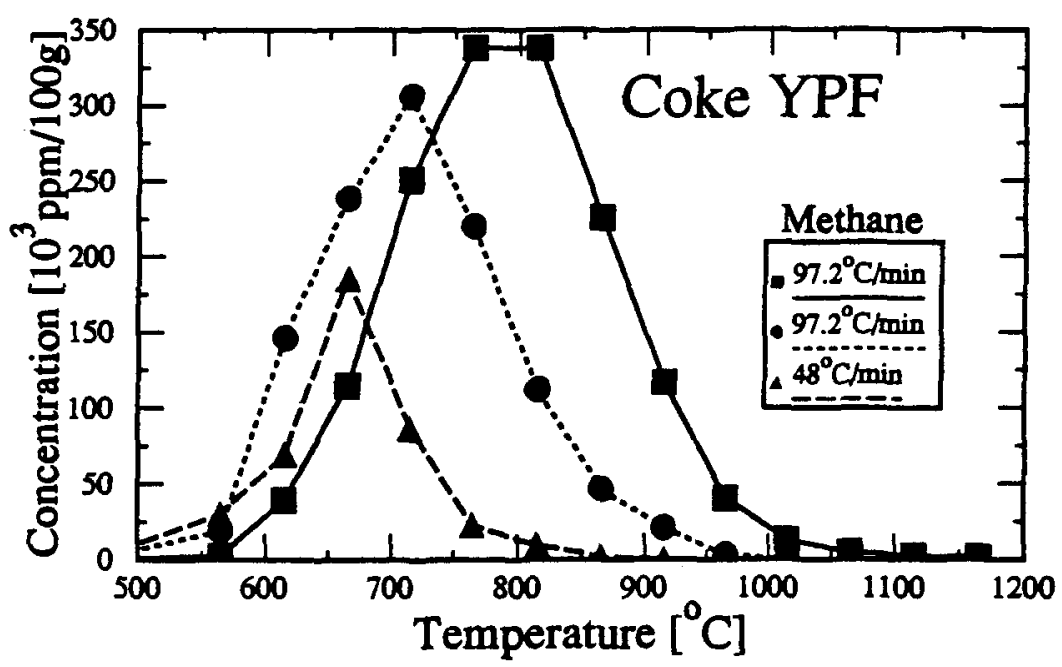

Annexel.4 : Effet du taux de chauffage sur le méthane du coke YPF, en milieu neutre ( $100 \%$ azote). 


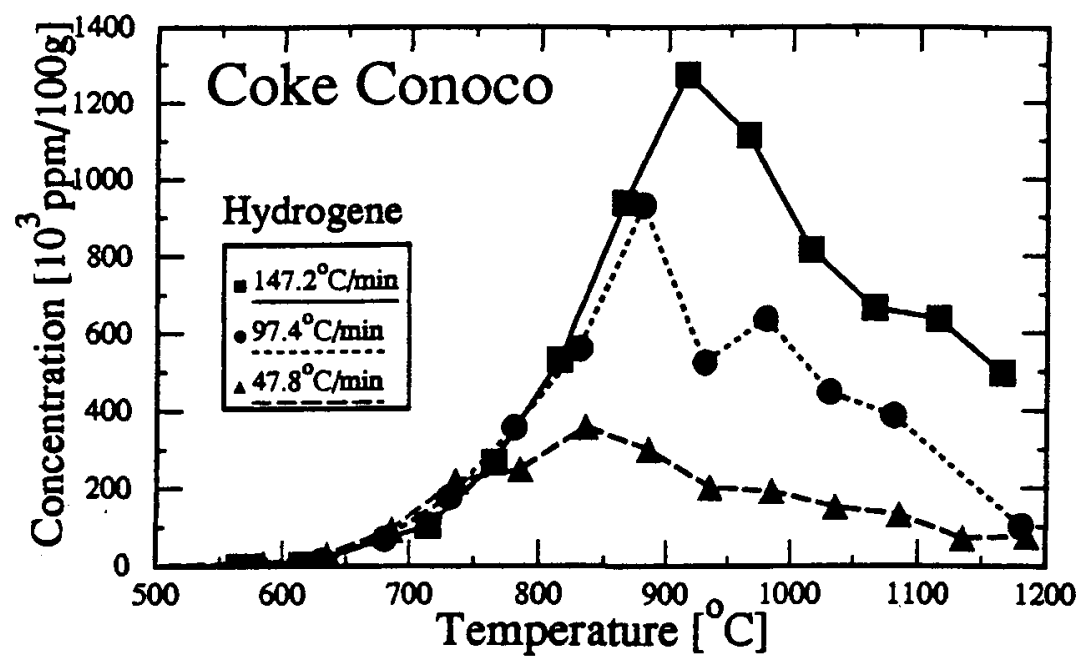

Annexel.5 : Effet du taux de chauffage sur l'hydrogène du coke CONOCO, en milieu neutre (100\% azote).

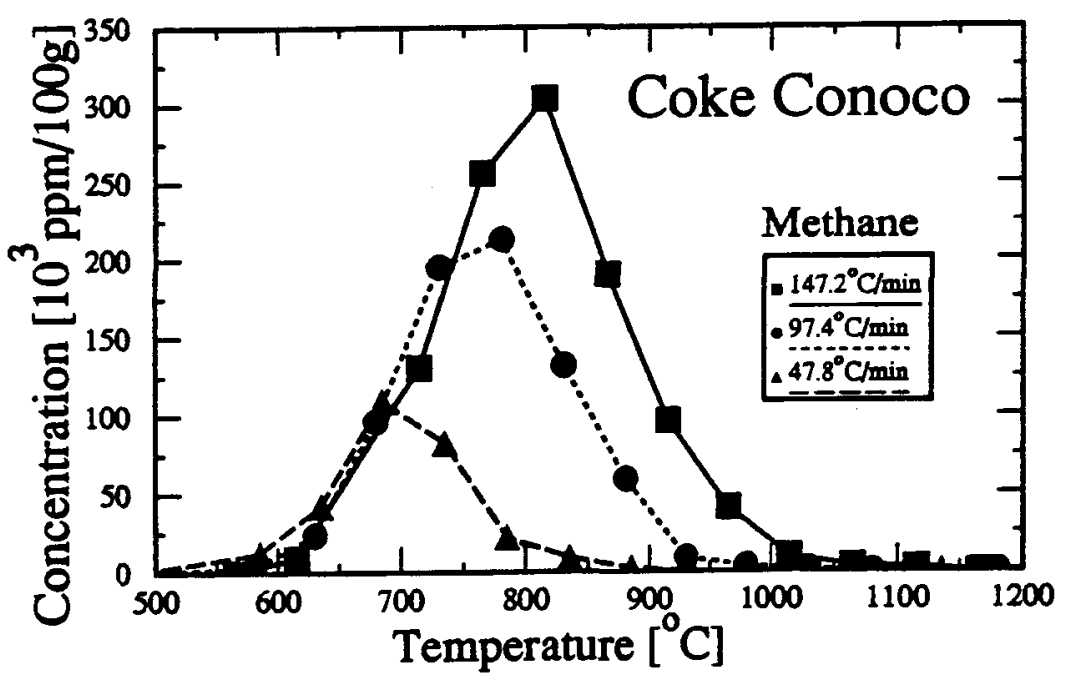

Annexel.6 : Effet du taux de chauffage sur le méthane du coke CONOCO, en milleu neutre ( $100 \%$ azote). 
Anneze II : $\quad$ Dérivée du thermogramme, valeurs instantanées et cumulatives, relatives au coke TEXACO, à 100 et $50^{\circ} \mathrm{C} / \mathrm{min}$. 
Temperature $\left[{ }^{\circ} \mathrm{C}\right]$

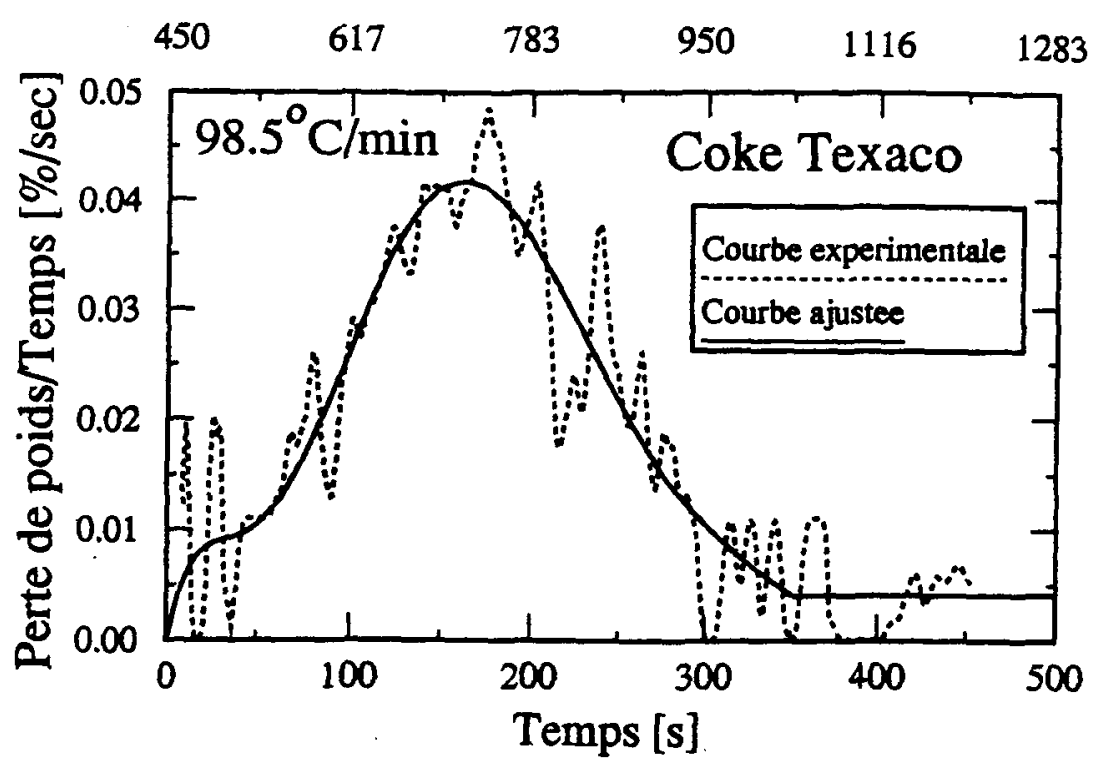

Annexell.1 : Dérivées du coke TEXACO à $100^{\circ} \mathrm{C} / \mathrm{min}$.

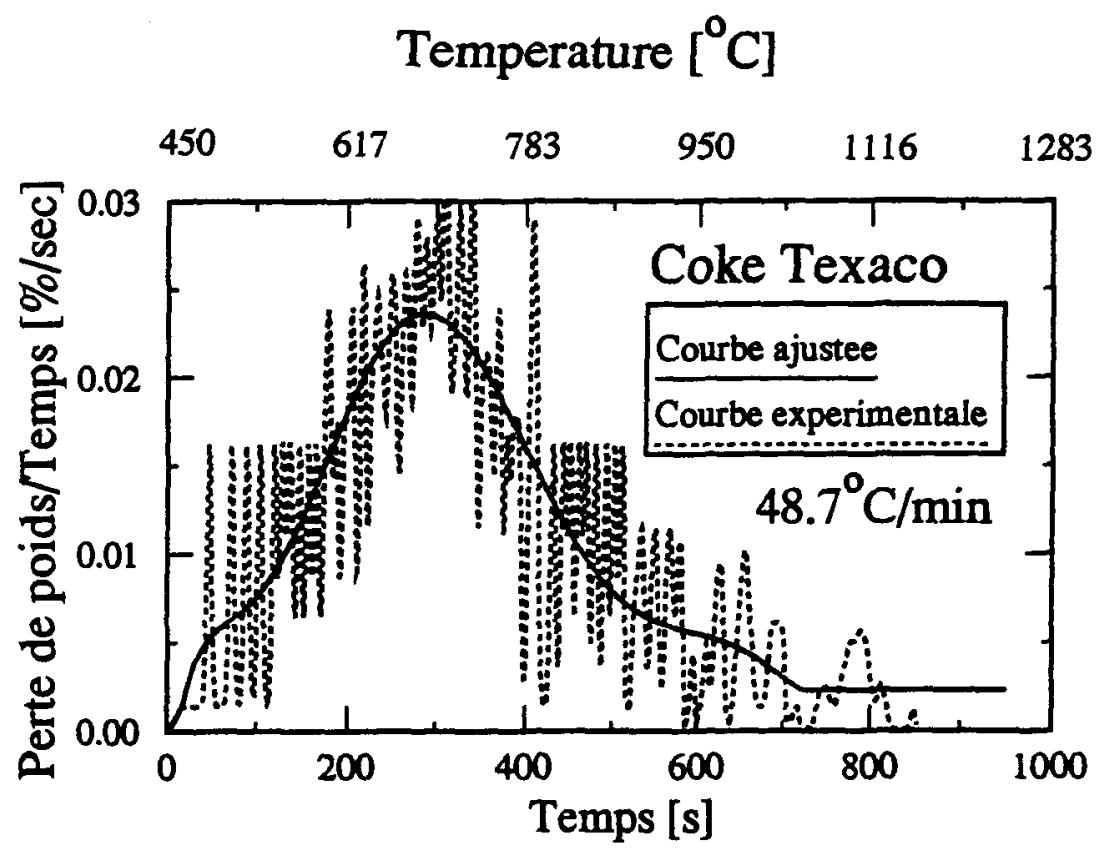

Annexell.2 : Dérivees du coke TEXACO à $50^{\circ} \mathrm{C} / \mathrm{min}$. 
Temperature $\left[{ }^{\circ} \mathrm{C}\right]$

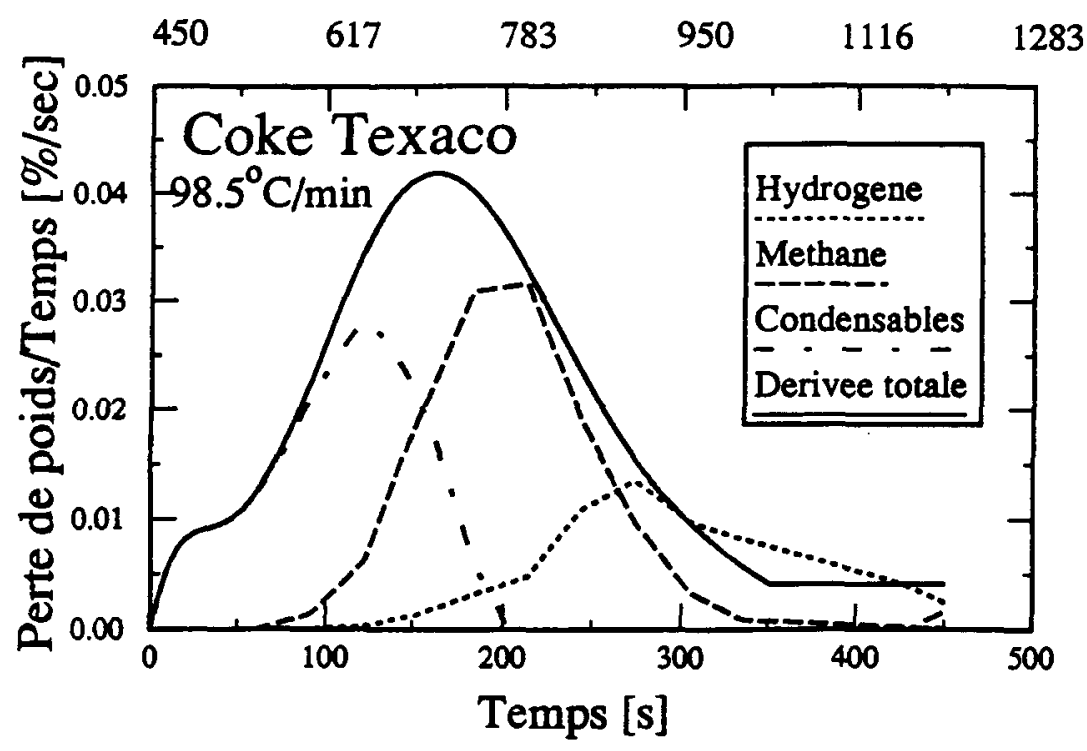

AnnexelI.3 : Valeurs instantanées du coke TEXACO à $100^{\circ} \mathrm{C} / \mathrm{min}$.

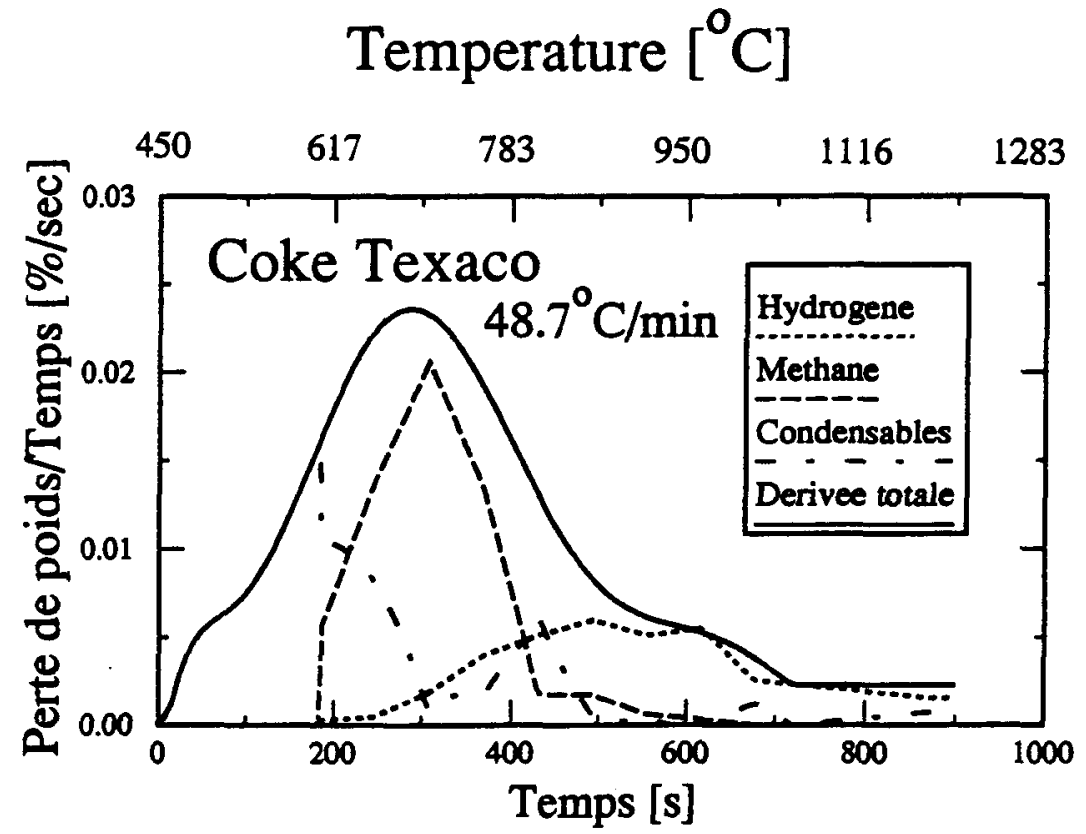

Annexell.4 : Valeurs instantanées du coke TEXACO à $50^{\circ} \mathrm{C} / \mathrm{min}$. 


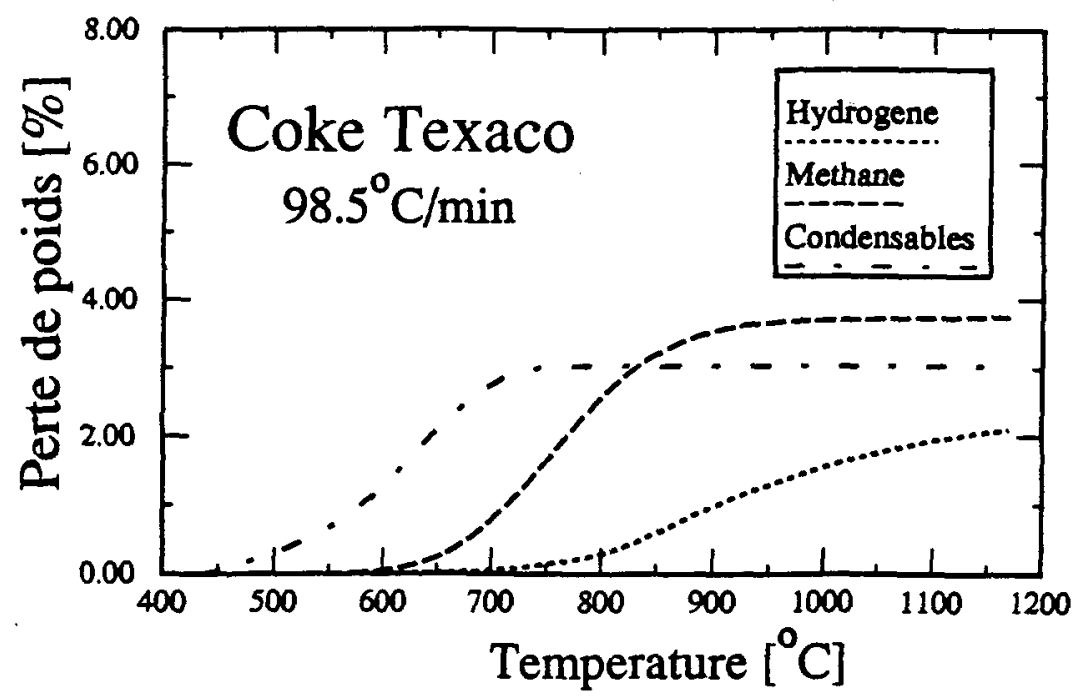

AnnexelI.5 : Valeurs cumulatives du coke TEXACO à $100^{\circ} \mathrm{C} / \mathrm{min}$.

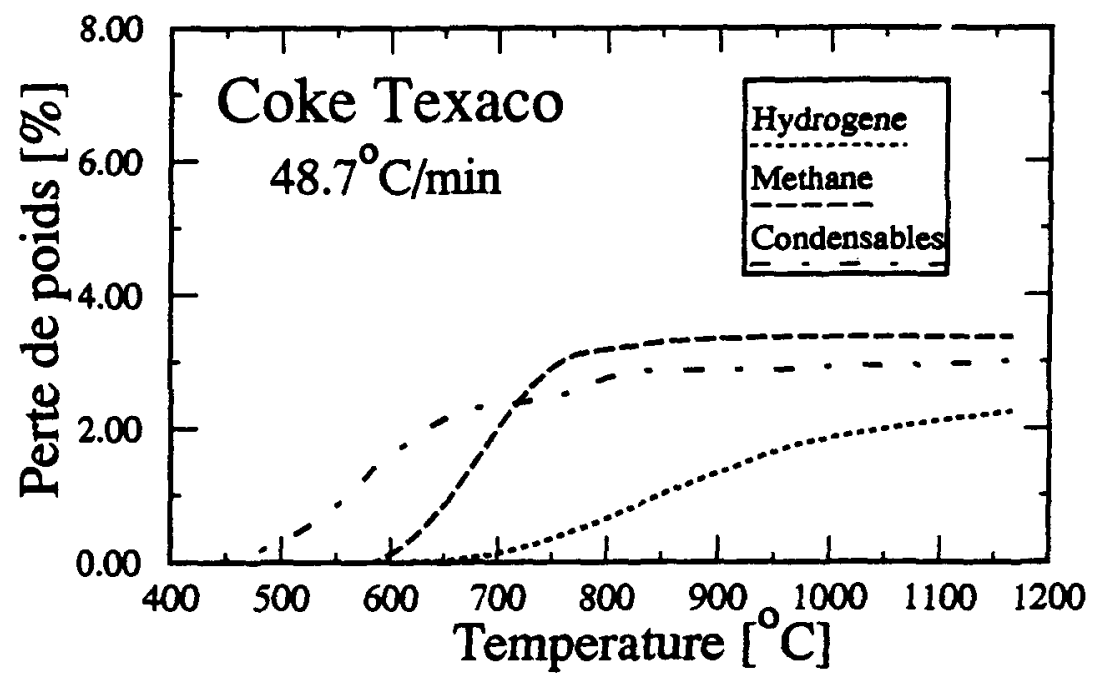

Annexell.6 : Valeurs cumulatives du coke TEXACO à $50^{\circ} \mathrm{C} / \mathrm{min}$. 
Annexe III : Comparaison des conversions expérimentales et issues du modèle pour le coke TEXACO, à 100 et $50^{\circ} \mathrm{C} / \mathrm{min}$. 


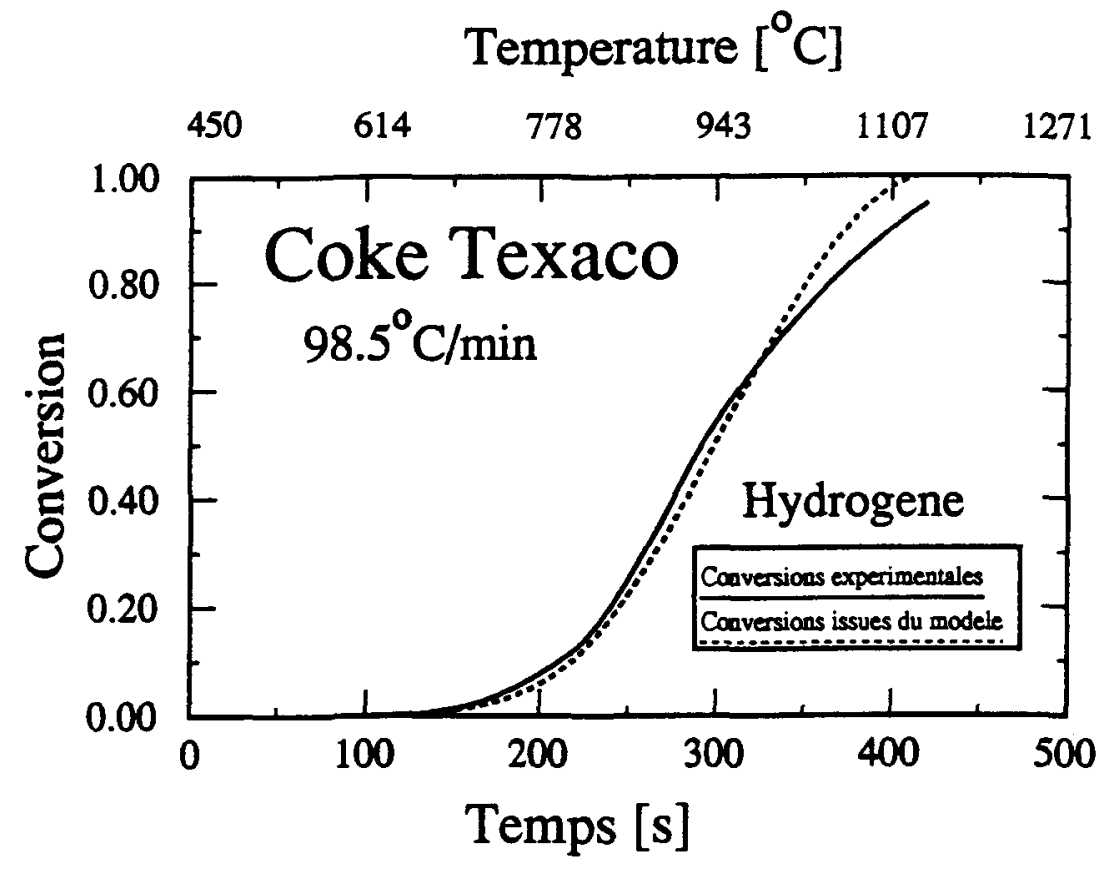

AnnexellI.1 : Conversions pour l'hydrogène du coke TEXACO à $100^{\circ} \mathrm{C} / \mathrm{min}$.

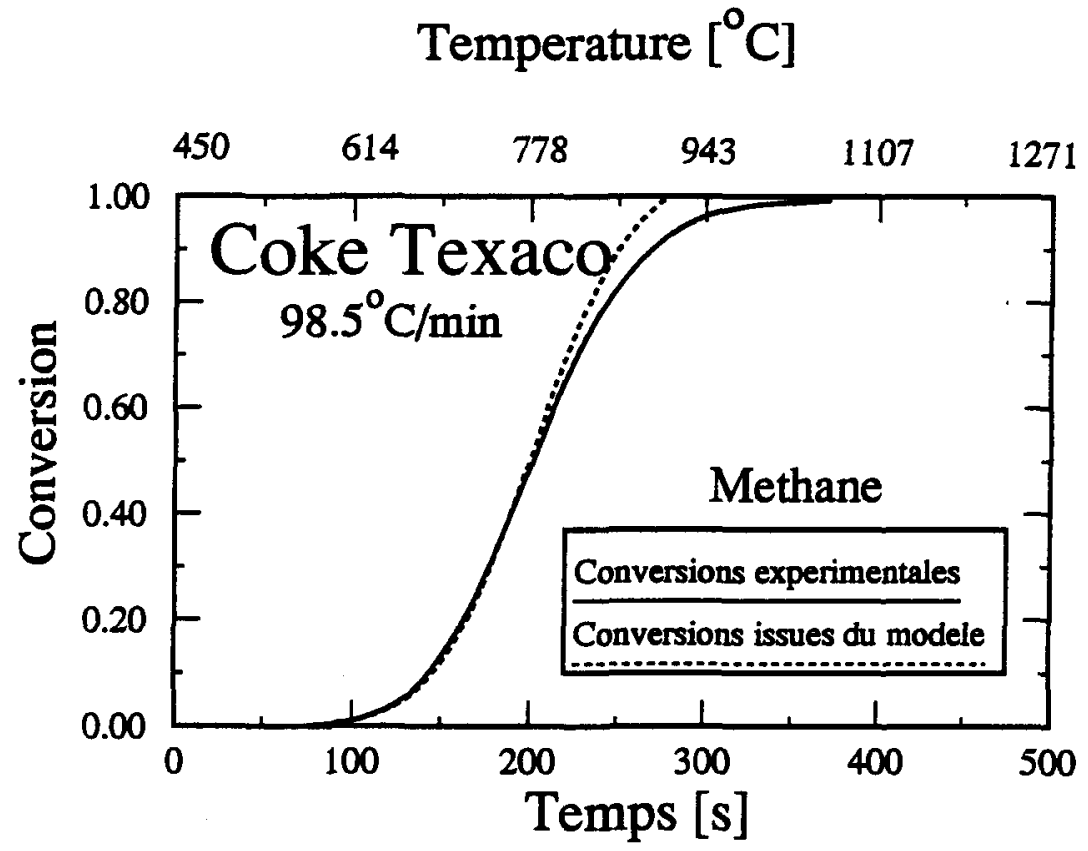

AnnexeIII.2 : Conversions pour le méthane du coke TEXACO à $100^{\circ} \mathrm{C} / \mathrm{min}$. 


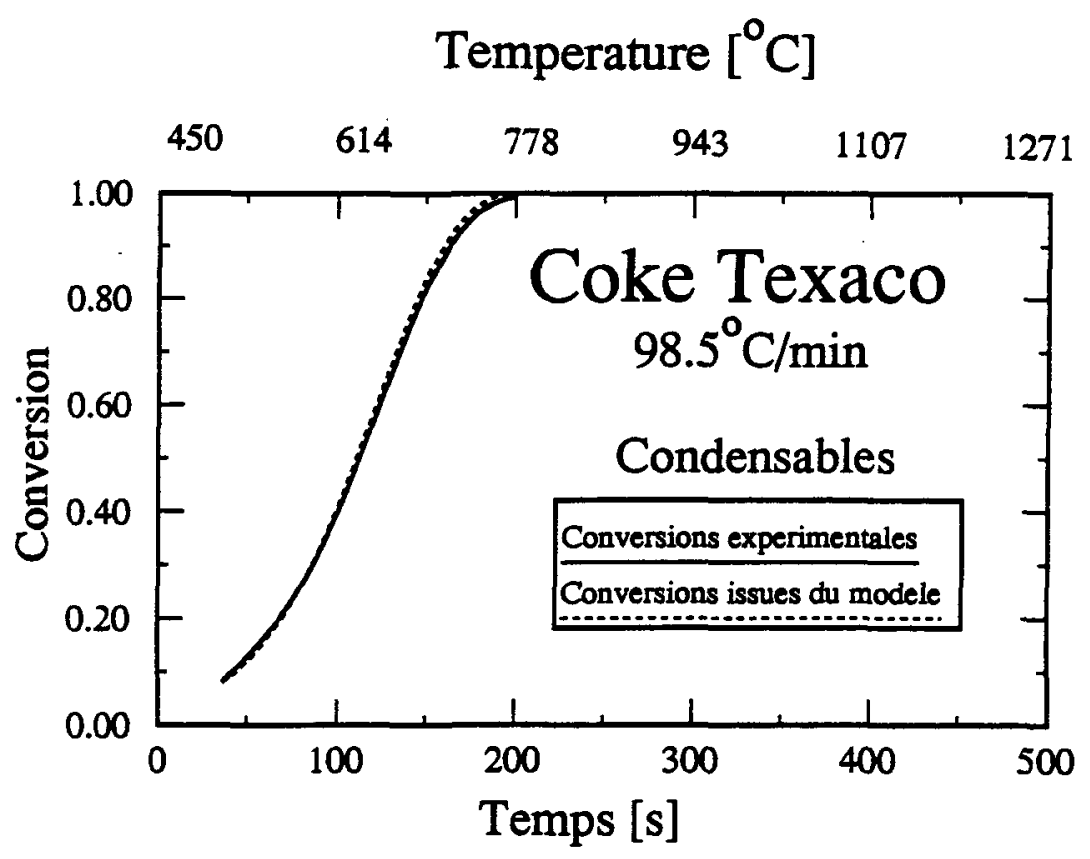

AnnexelII.3.: Conversions pour les condensables du coke TEXACO à $100^{\circ} \mathrm{C} / \mathrm{min}$.

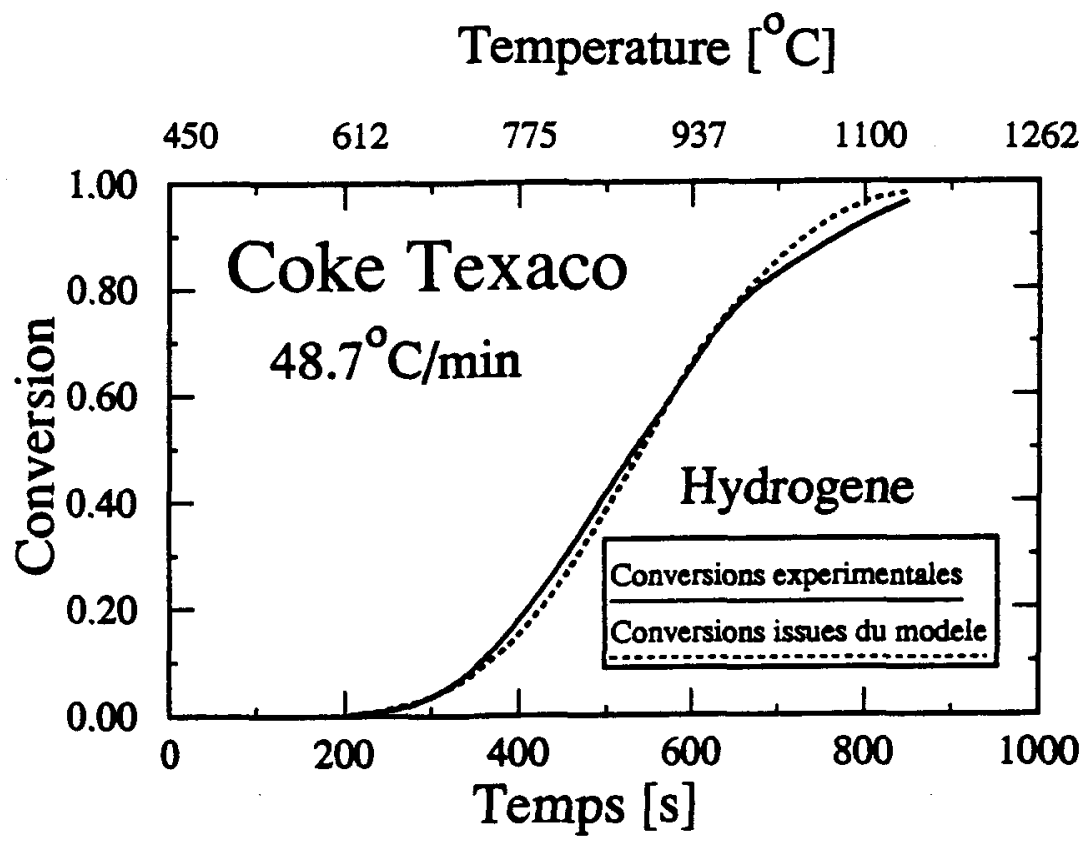

Annexelll.4 : Conversions pour l'hydrogène du coke TEXACO à $50^{\circ} \mathrm{C} / \mathrm{min}$. 


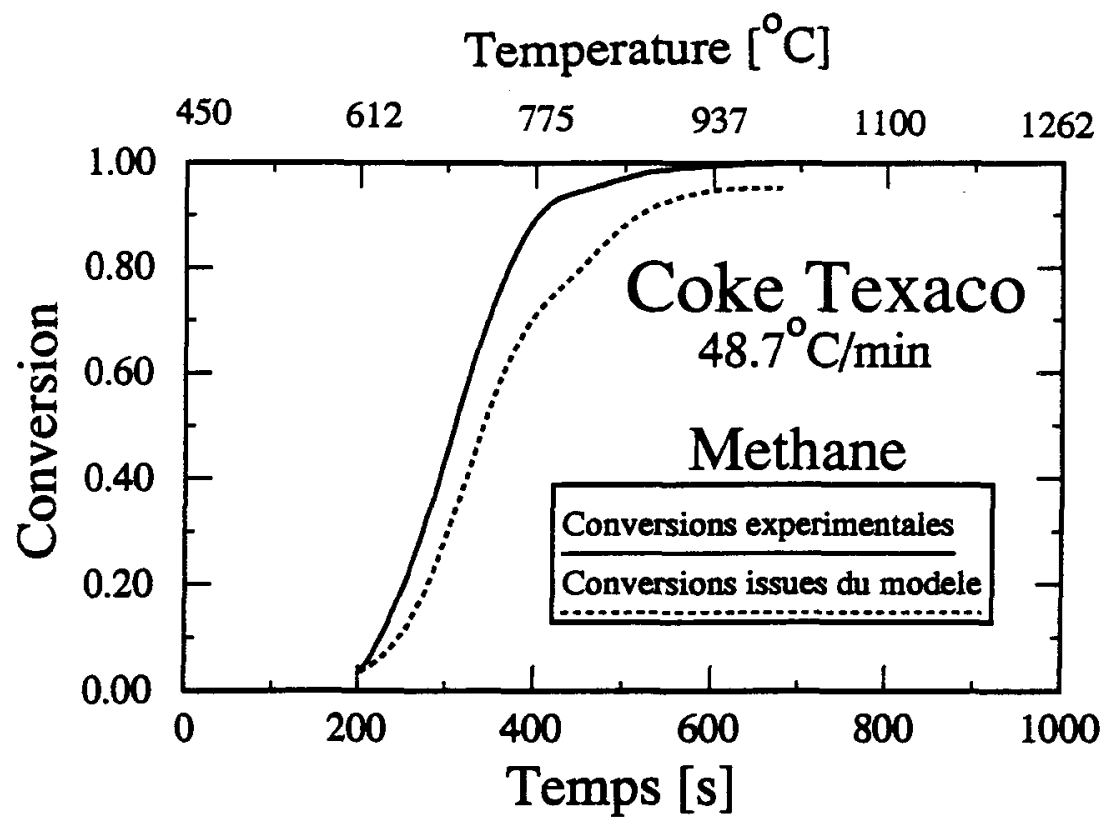

AnnexeIII.5 : Conversions pour le méthane du coke TEXACO à $50^{\circ} \mathrm{C} / \mathrm{min}$.

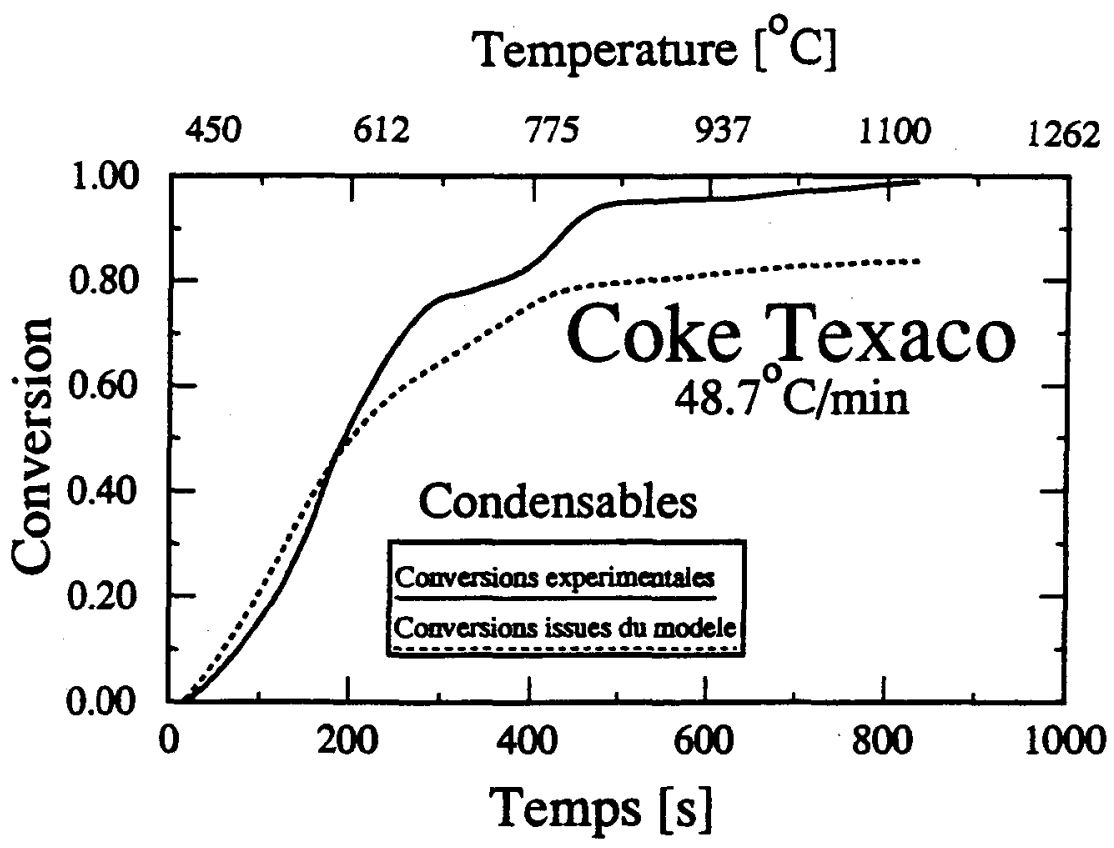

Annexelli.6.: Conversions pour les condensables du coke TEXACO à $50^{\circ} \mathrm{C} / \mathrm{min}$. 
Annexe IV : Comparaison des thermogrammes en milieux neutre et oxydant, pour les trois autres cokes (RTW, YPF et CONOCO). 


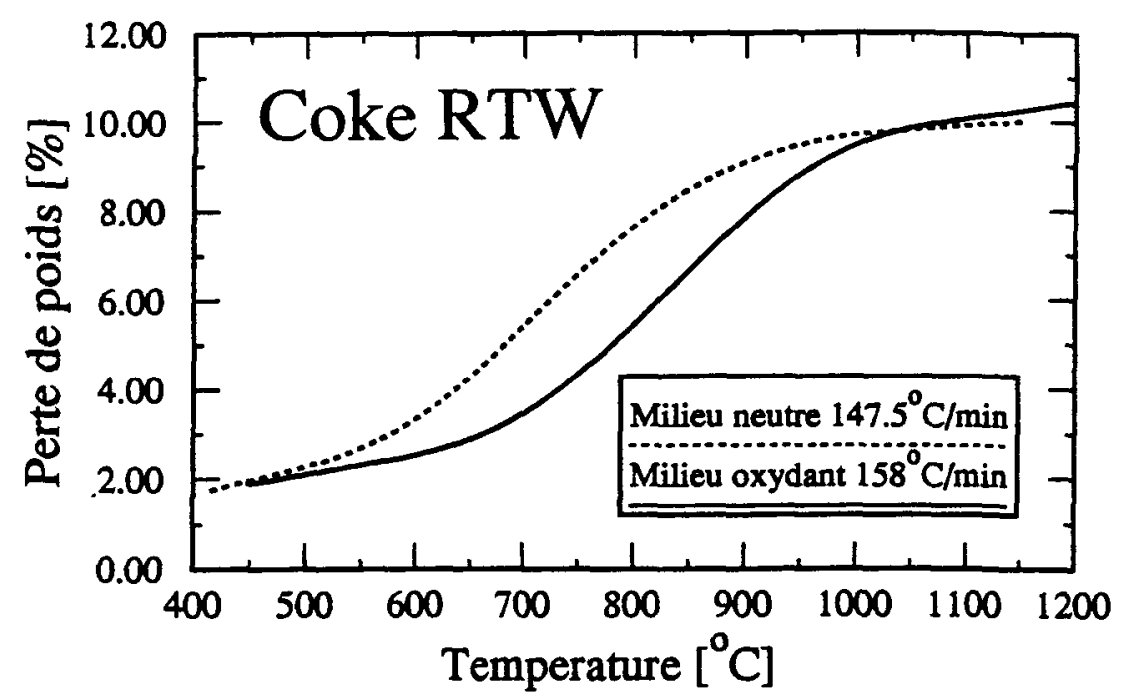

AnnexeIV.1 : Thermogrammes du coke RTW à $150^{\circ} \mathrm{C} / \mathrm{min}$.

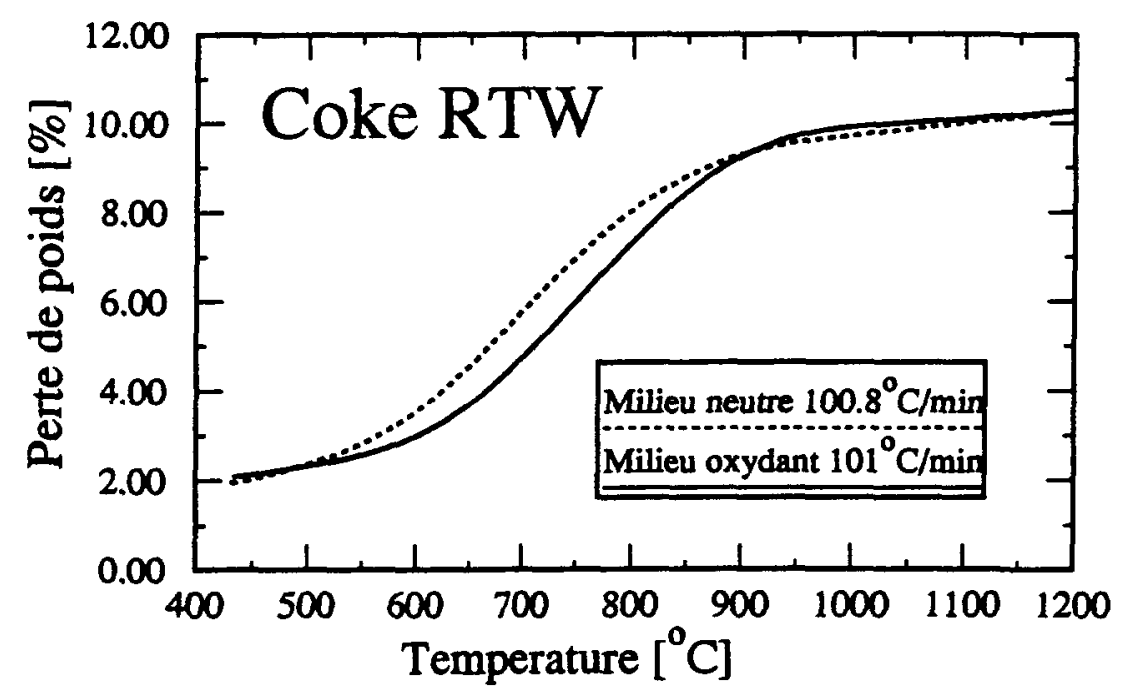

AnnexeIV.2 : Thermogrammes du coke RTW à $100^{\circ} \mathrm{C} / \mathrm{min}$. 


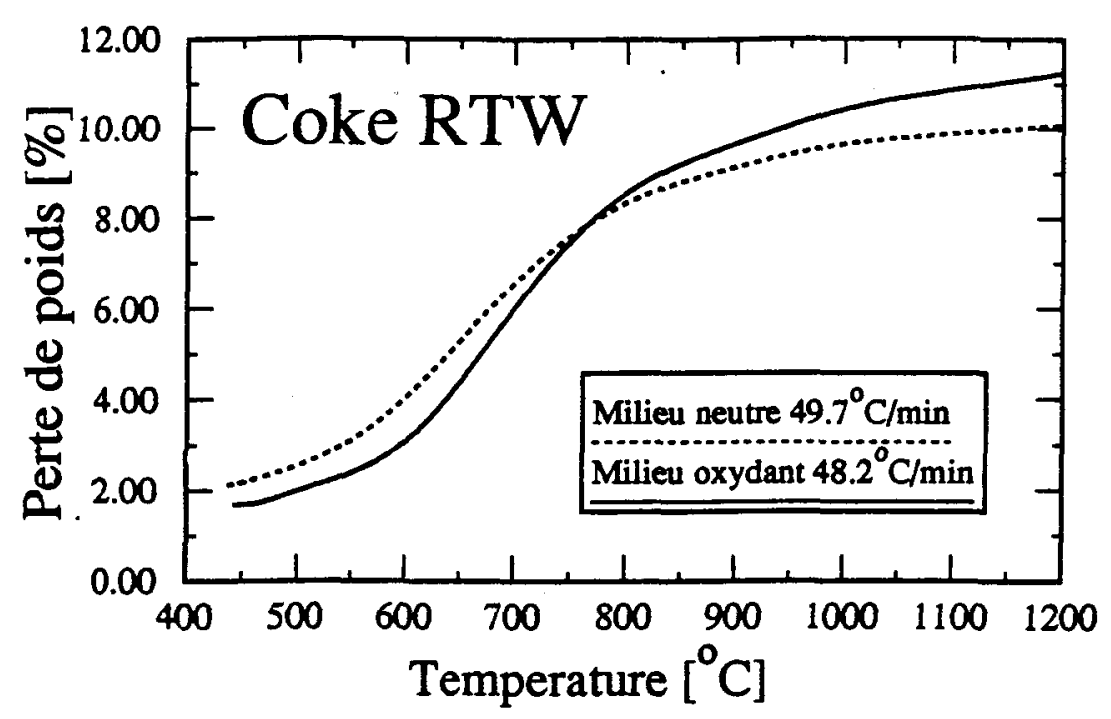

AnnexeIV.3 : Thermogrammes du coke $\mathrm{RTW}$ à $50^{\circ} \mathrm{C} / \mathrm{min}$.

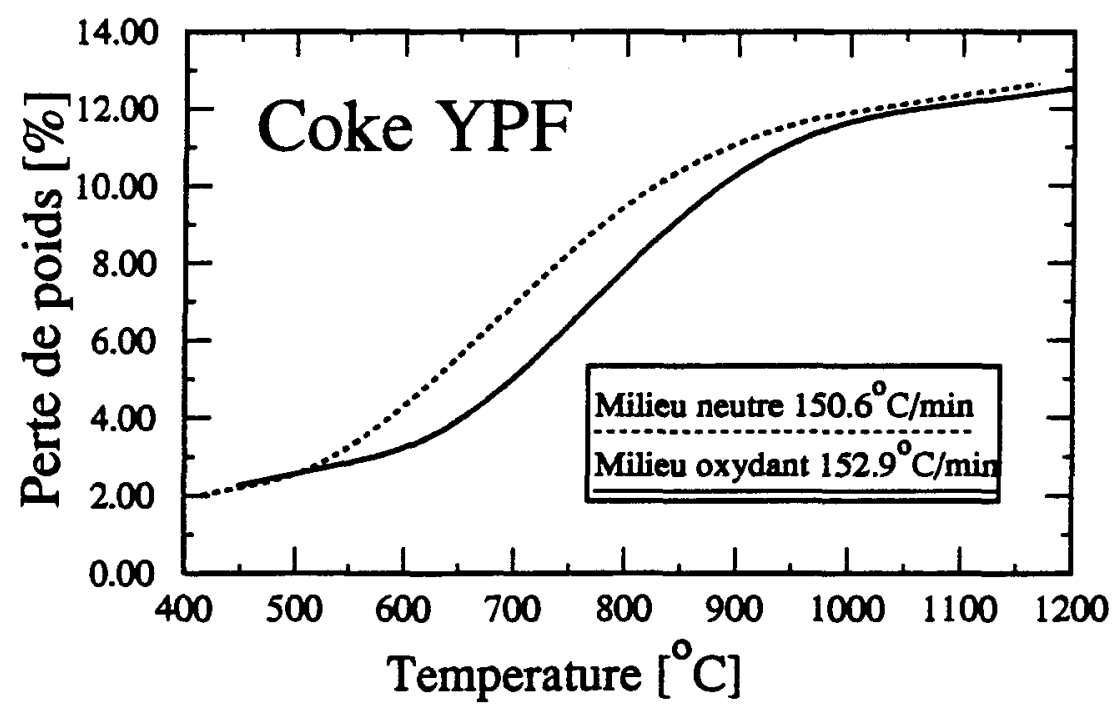

AnnexeIV.4 : Thermogrammes du coke YPF à $150^{\circ} \mathrm{C} / \mathrm{min}$. 


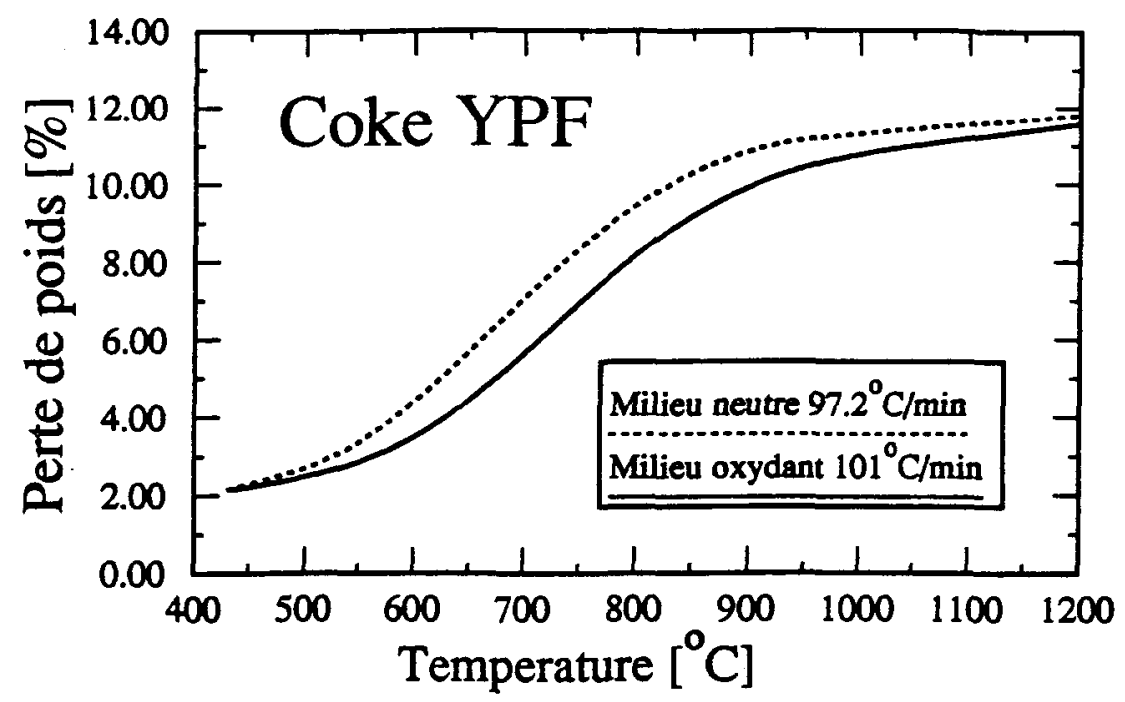

AnnexeIV.5 : Thermogrammes du coke YPF à $100^{\circ} \mathrm{C} / \mathrm{min}$.

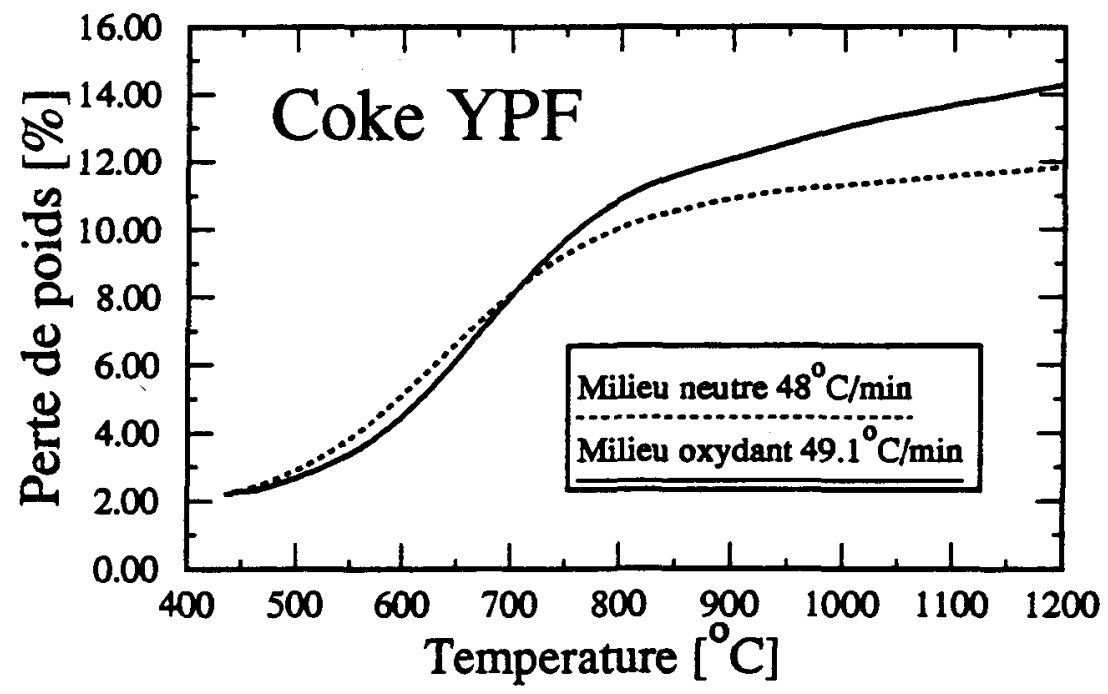

AnnexeIV.6 : Thermogrammes du coke YPF à $50^{\circ} \mathrm{C} / \mathrm{min}$. 


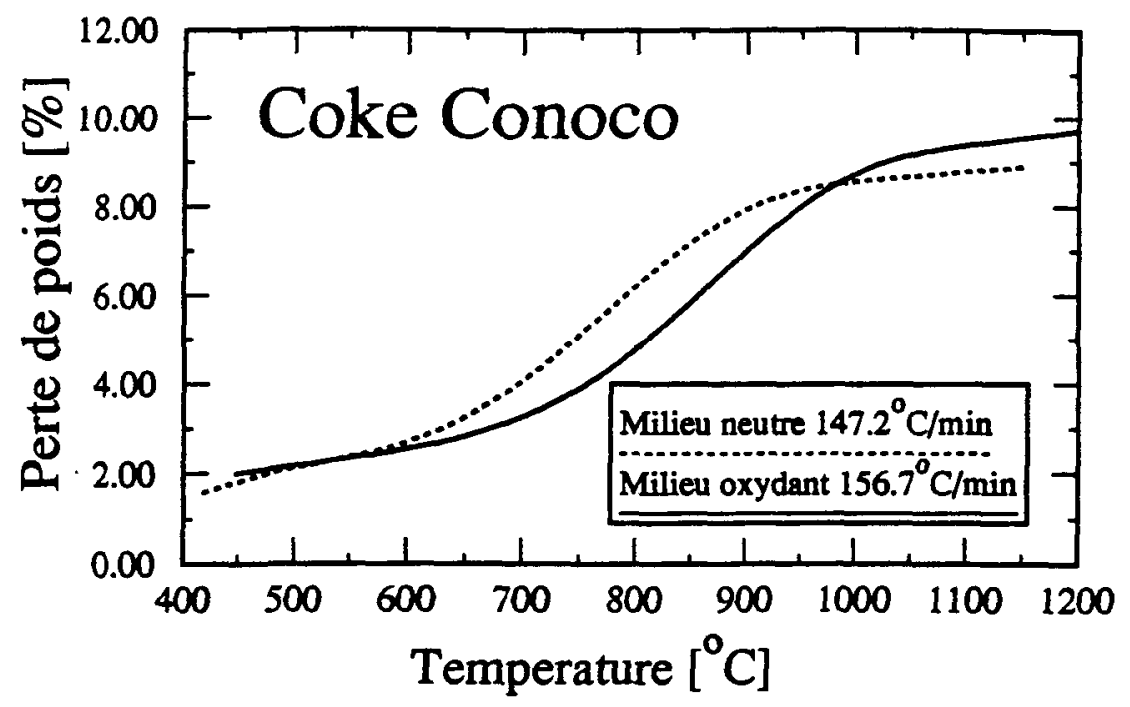

AnnexeIV.7 : Thermogrammes du coke CONOCO à $150^{\circ} \mathrm{C} / \mathrm{min}$.

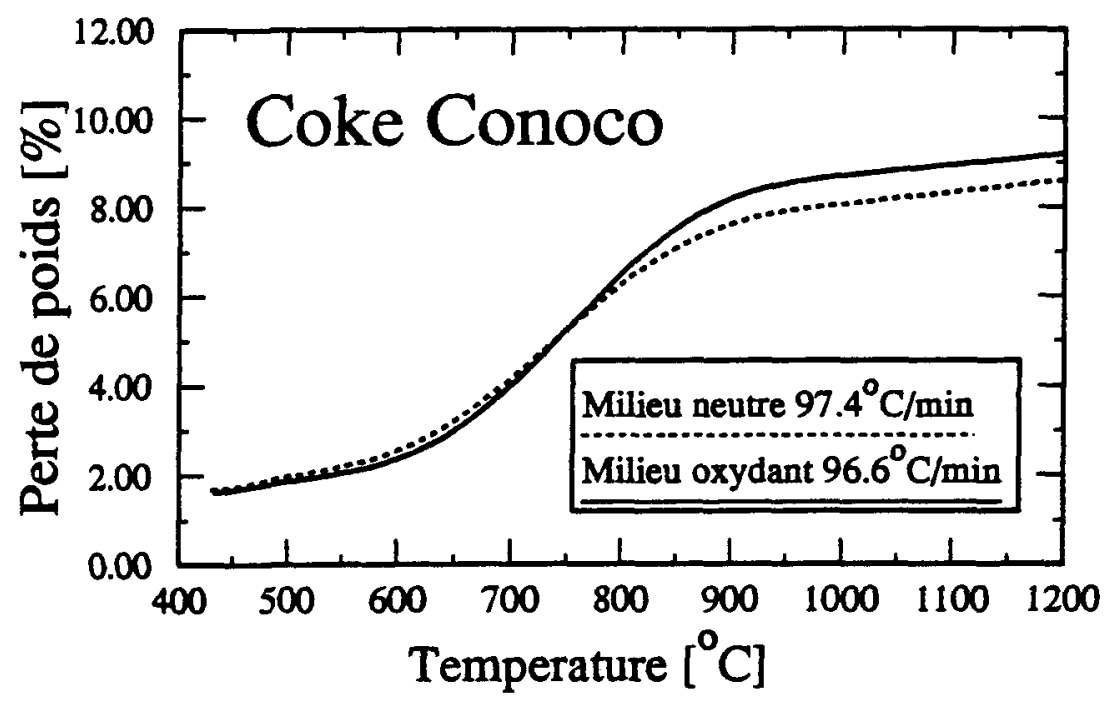

AnnexeIV.8 : Thermogrammes du coke CONOCO à $100^{\circ} \mathrm{C} / \mathrm{min}$. 


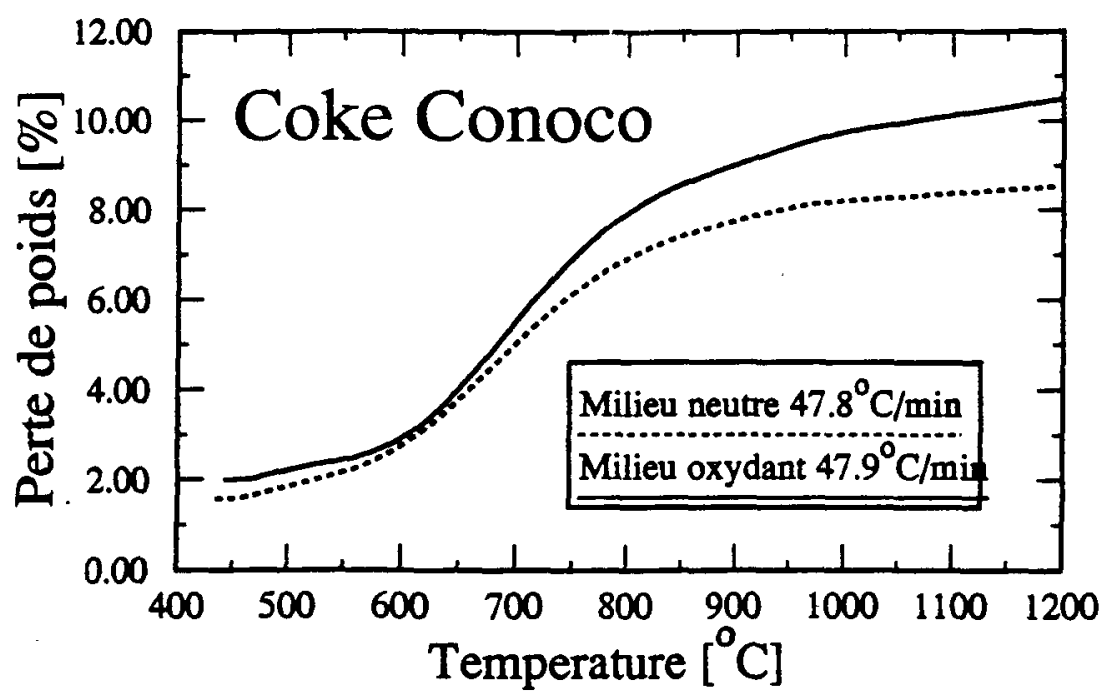

AnnexelV.9 : Thermogrammes du coke CONOCO à $50^{\circ} \mathrm{C} / \mathrm{min}$. 


\section{Annexe V: $\quad$ Comparaison des valeurs instantanées en milieux neutre et} oxydant, pour les trois autres cokes (RTW, YPF et CONOCO). 
Temperature $\left[{ }^{\circ} \mathrm{C}\right]$

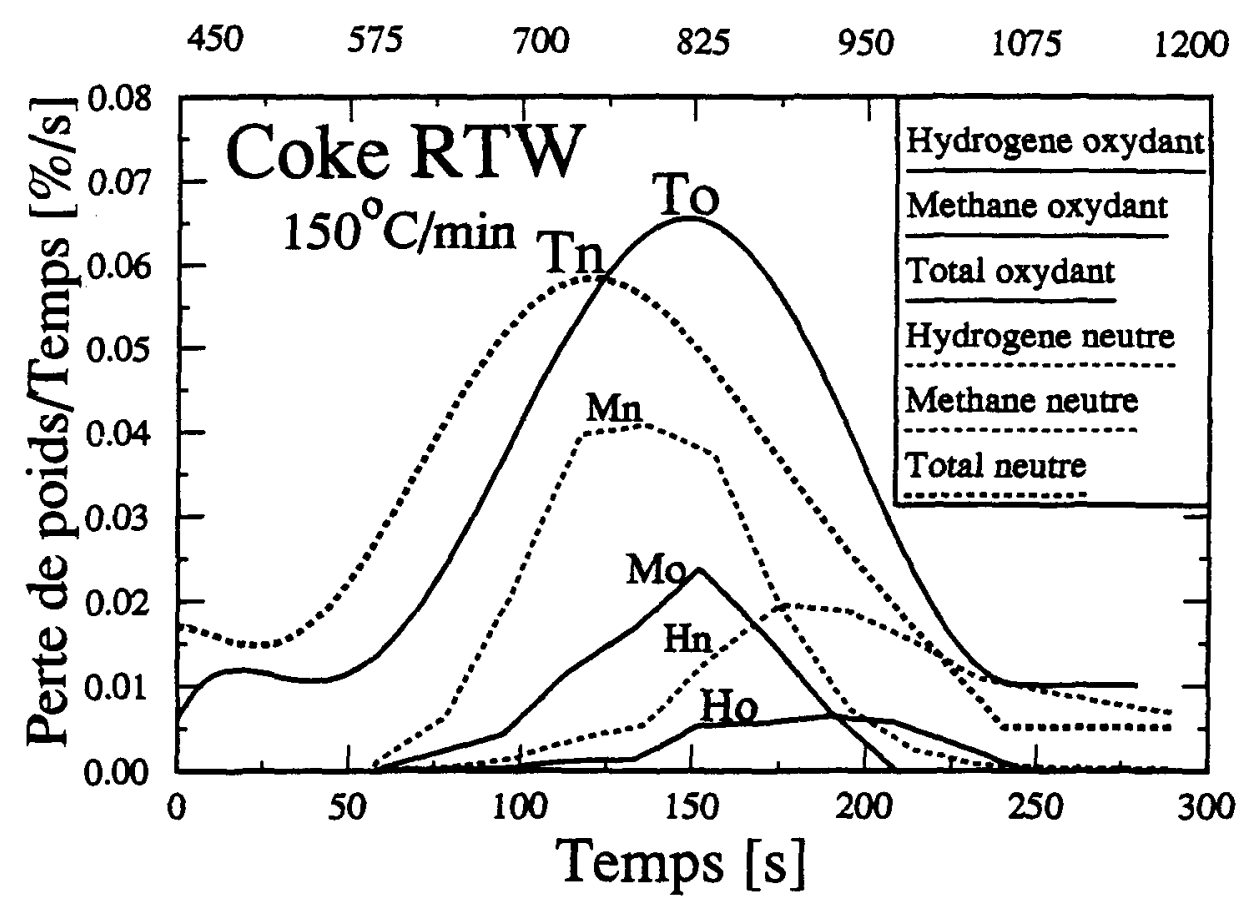

AnnexeV.1.: Valeurs instantanées du coke RTw à $150^{\circ} \mathrm{C} / \mathrm{min}$.

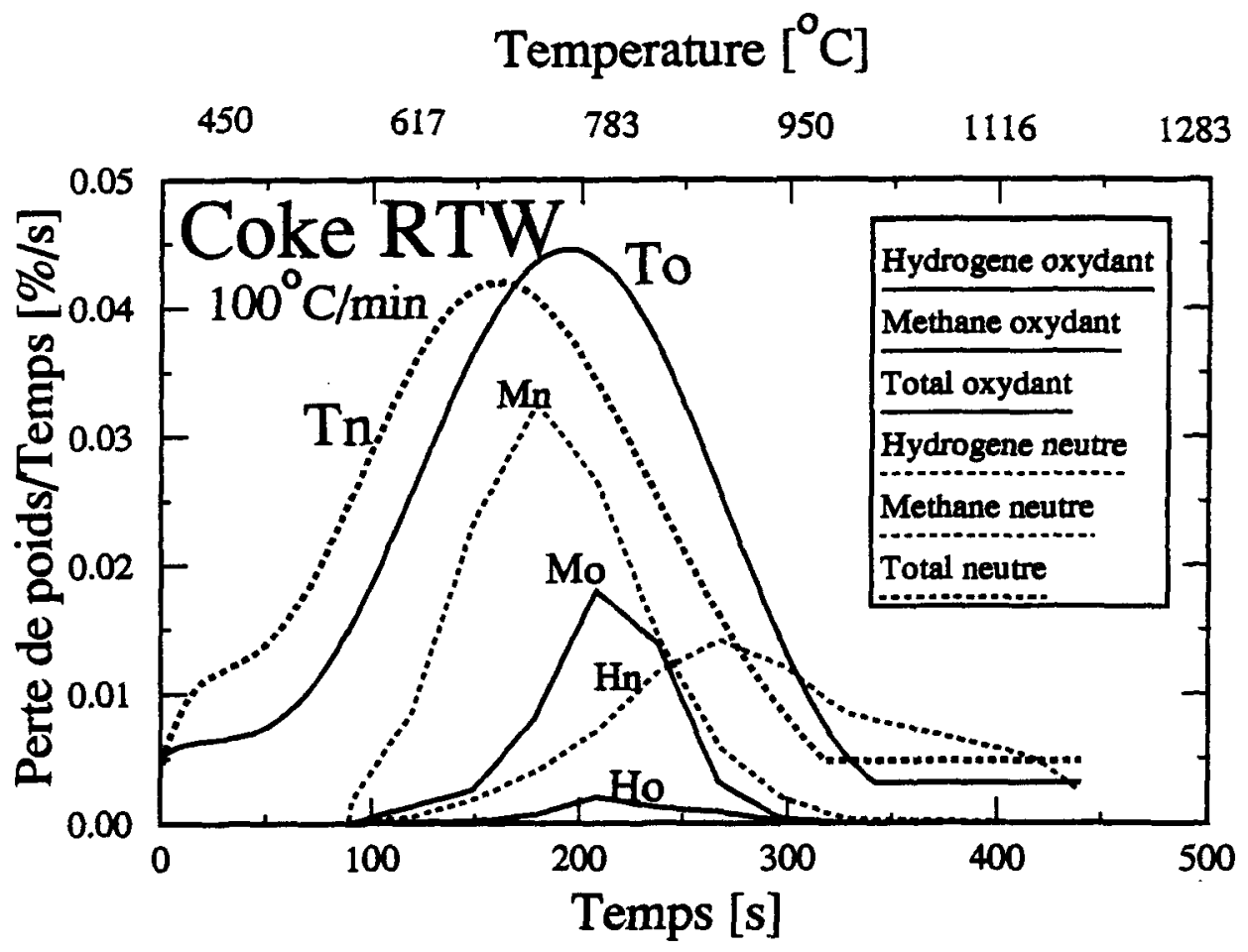

AnnexeV.2.: Valeurs instantanées du coke $\mathrm{RTW}$ à $100^{\circ} \mathrm{C} / \mathrm{min}$. 


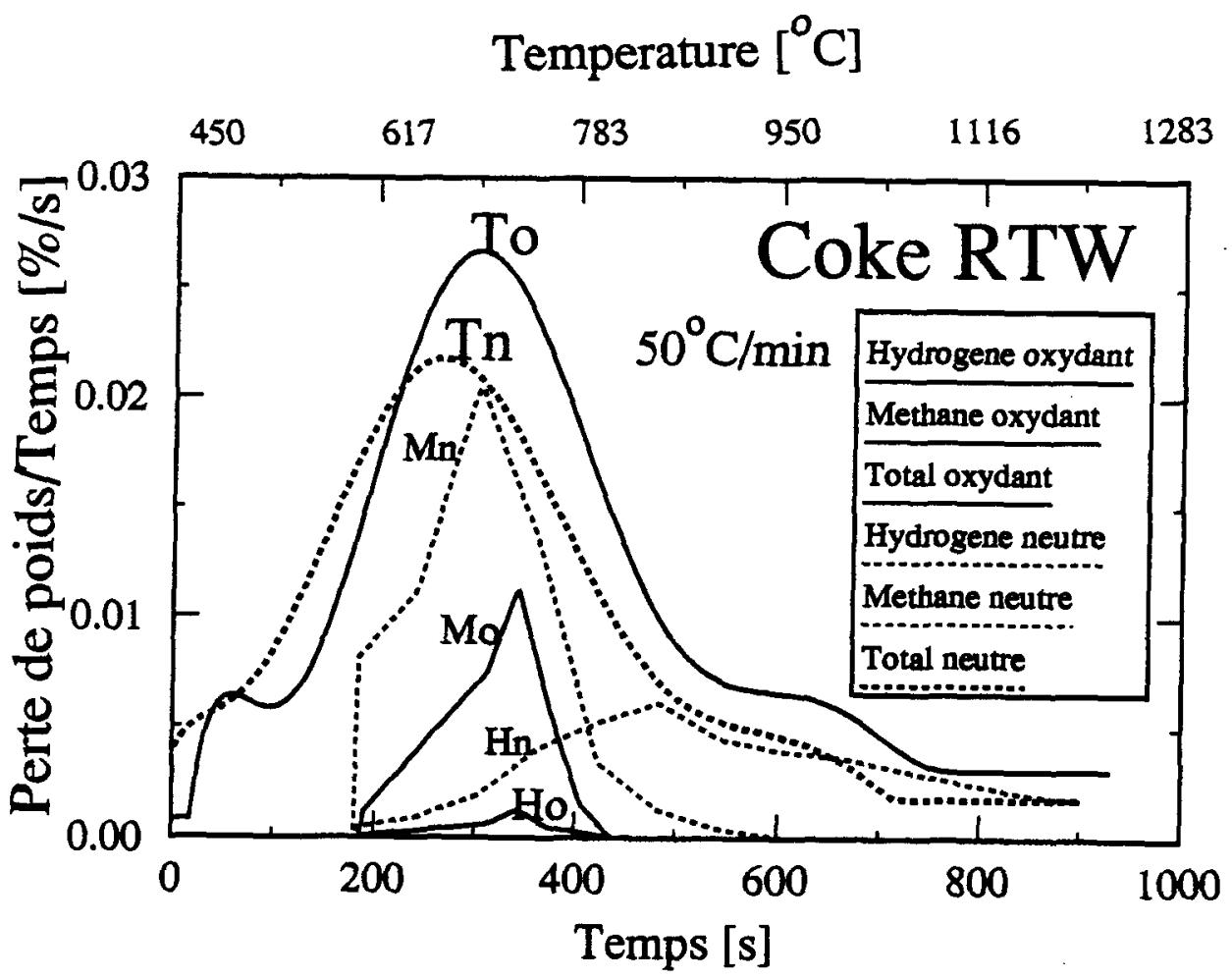

AnnexeV.3.: Valeurs instantanées du coke $R T W$ à $50^{\circ} \mathrm{C} / \mathrm{min}$.

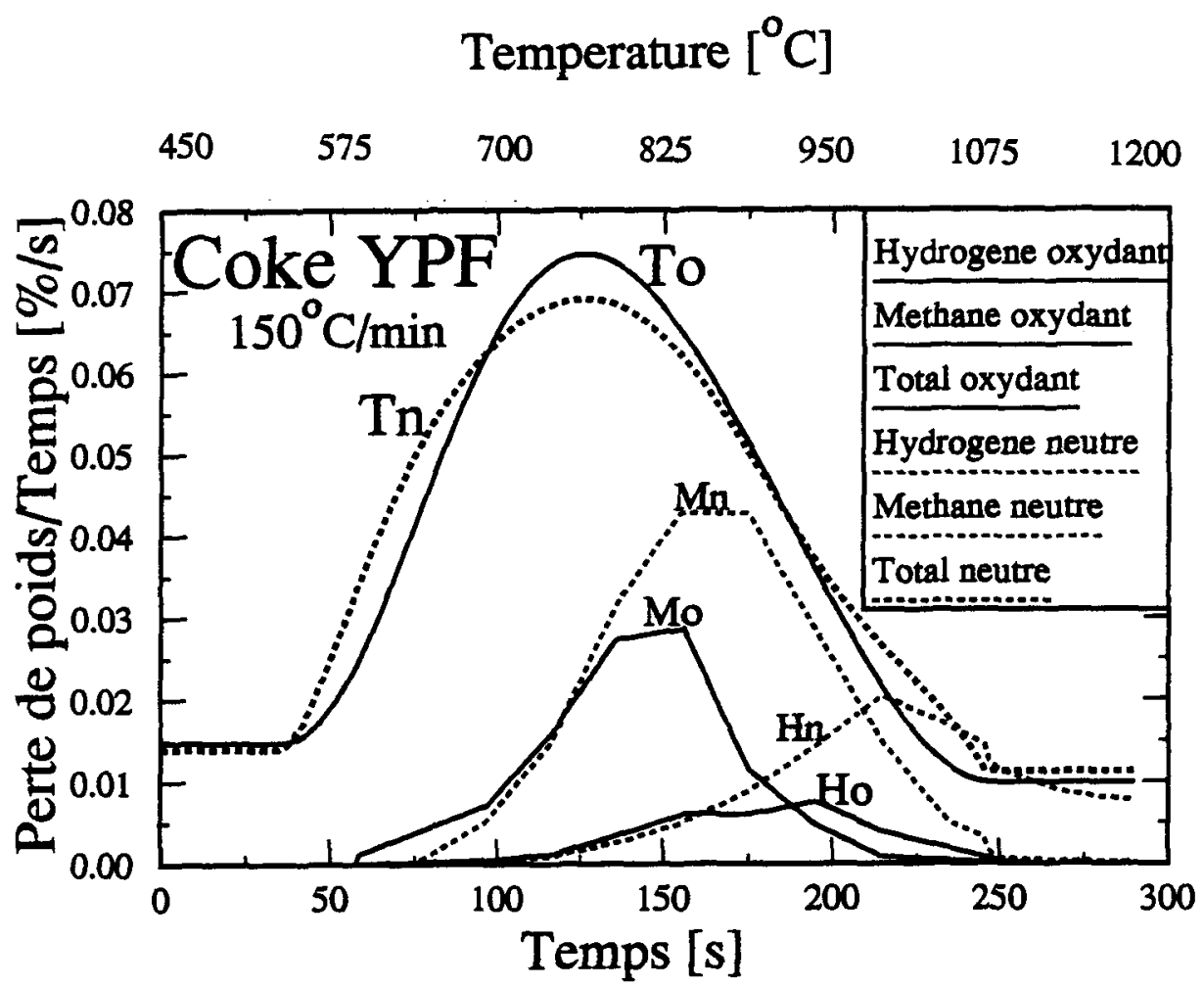

AnnexeV.4.: Valeurs instantanées du coke YPF à $150^{\circ} \mathrm{C} / \mathrm{min}$. 


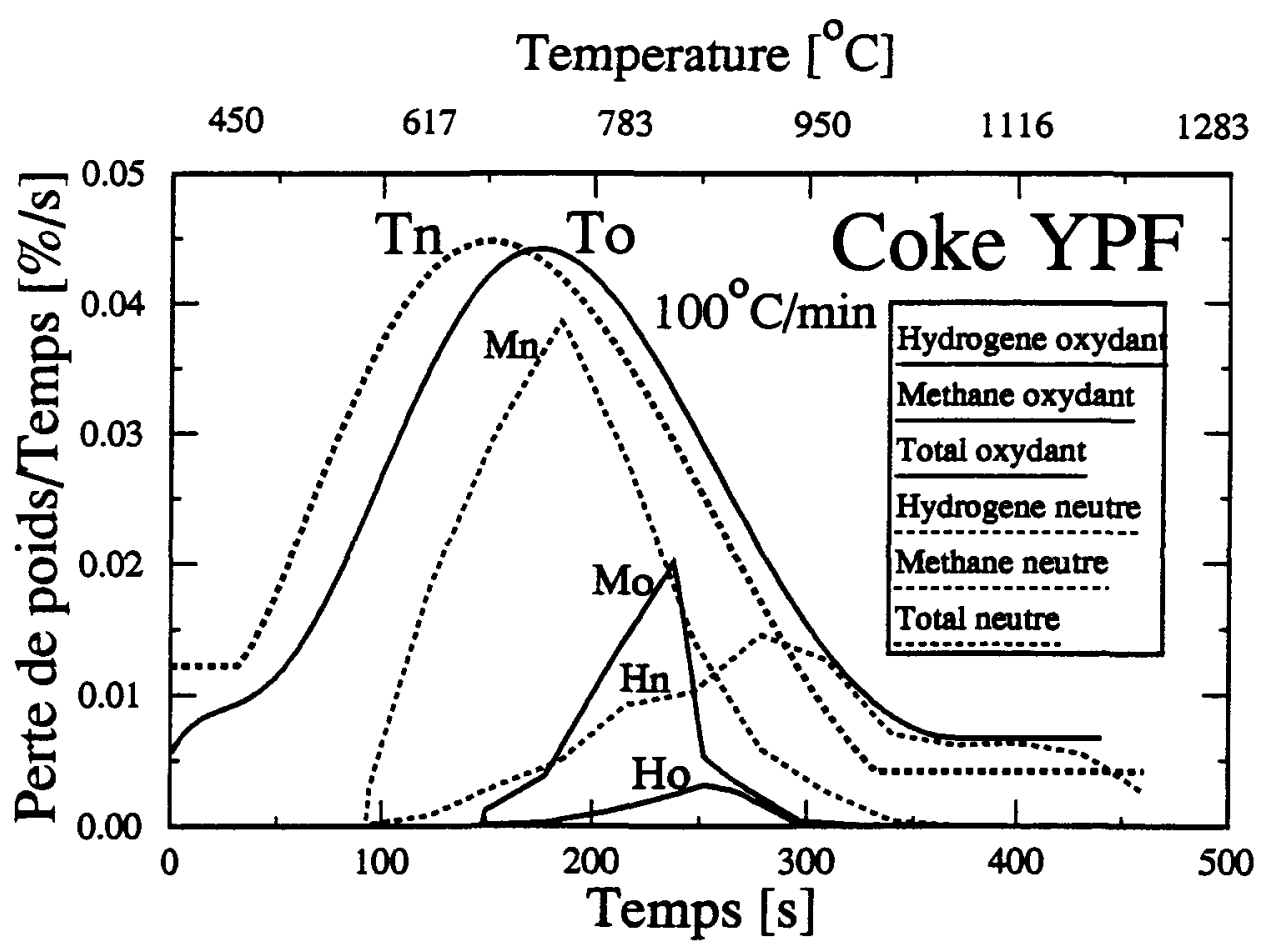

AnnexeV.5.: Valeurs instantanées du coke YPF à $100^{\circ} \mathrm{C} / \mathrm{min}$.

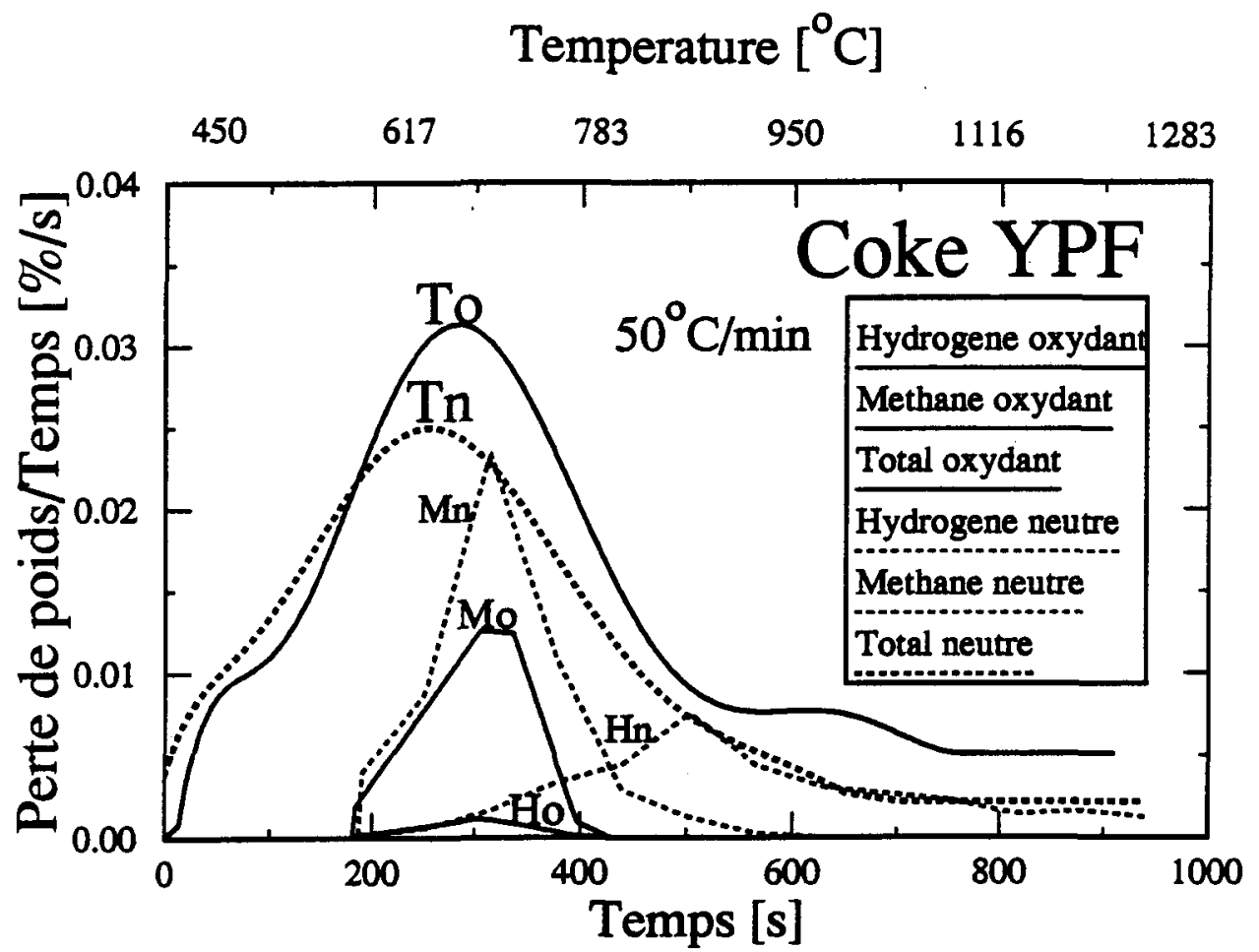

AnnexeV.6.: Valeurs instantanées du coke YPF à $50^{\circ} \mathrm{C} / \mathrm{min}$ 


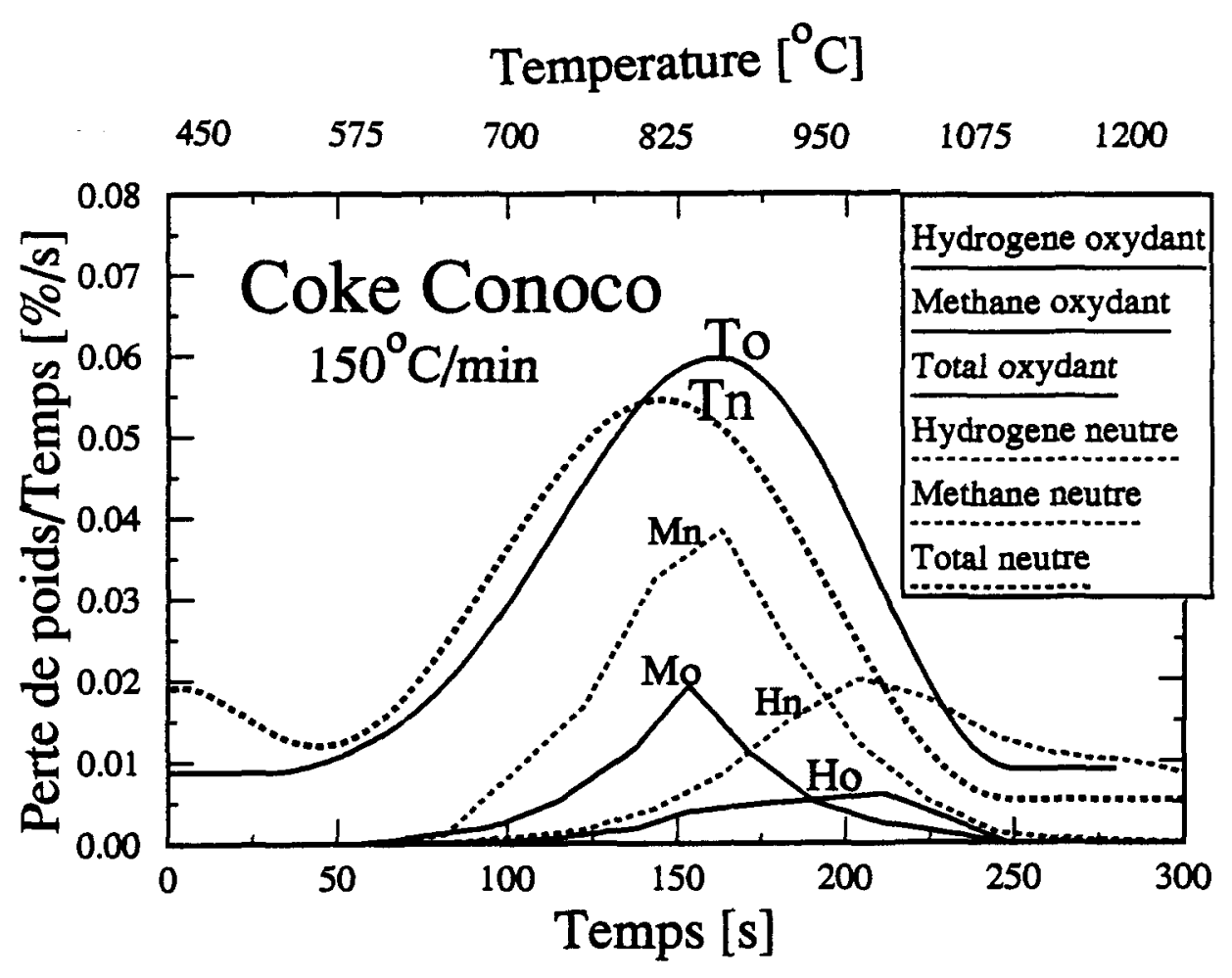

AnnexeV.7.: Valeurs instantanées du coke CONOCO à $150^{\circ} \mathrm{C} / \mathrm{min}$.

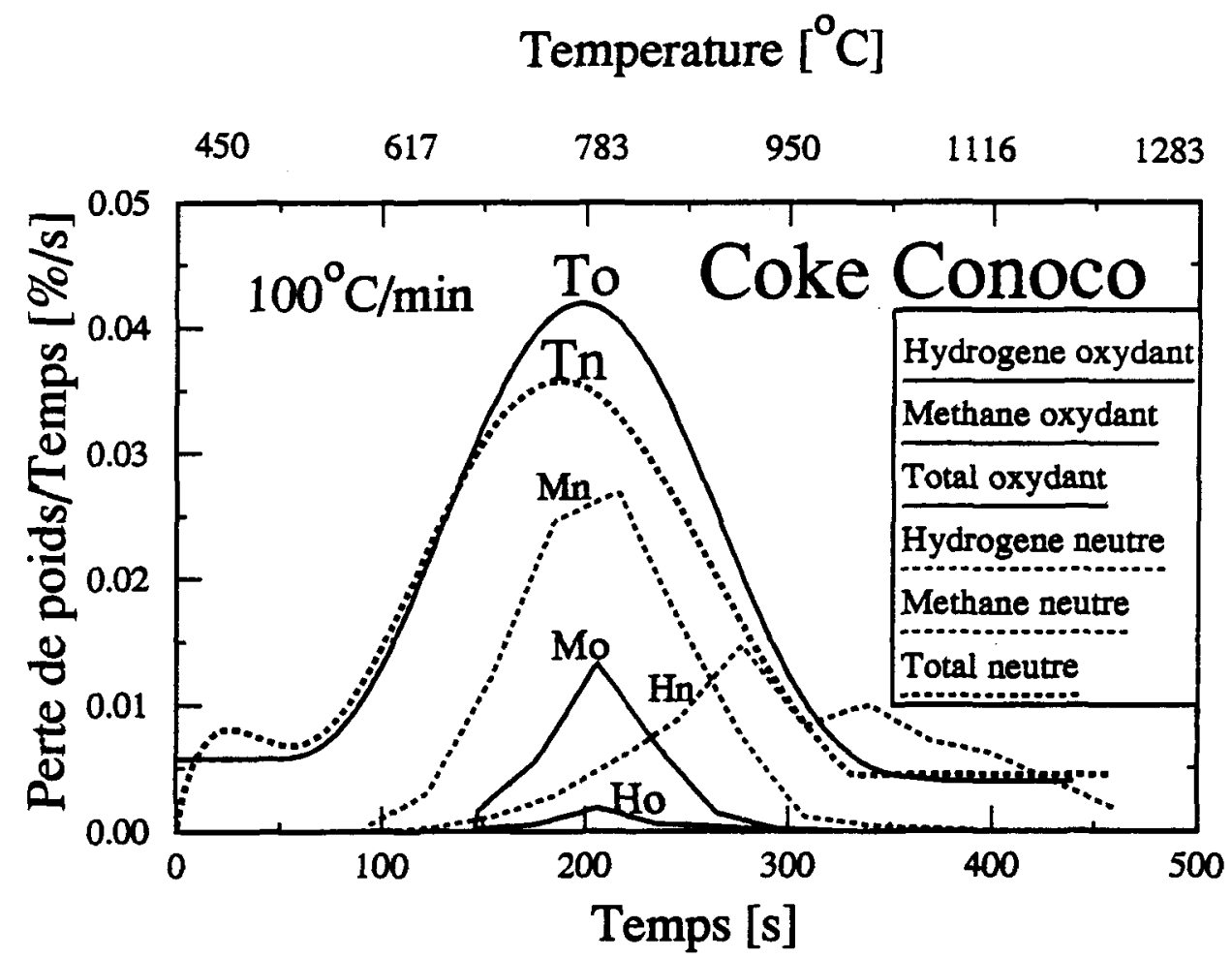

AnnexeV.8.: Valeurs instantanées du coke CONOCO à $100^{\circ} \mathrm{C} / \mathrm{min}$. 


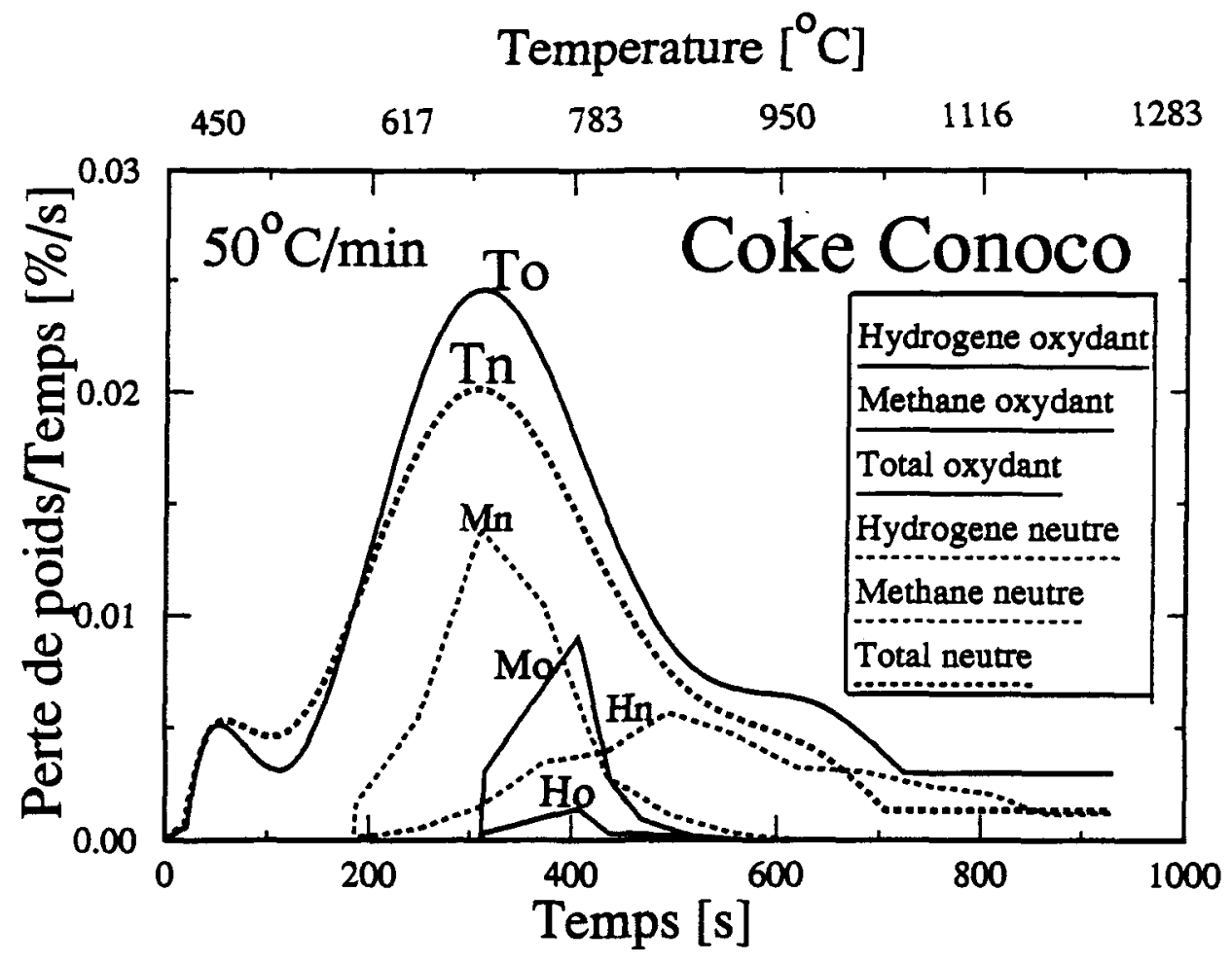

AnnexeV.9.: Valeurs instantanées du coke CONOCO à $50^{\circ} \mathrm{C} / \mathrm{min}$. 
Anneze VI: Combustion de l'hydrogène et du méthane pour les trois autres cokes (RTW, YPF et CONOCO) en milieu oxydant. 


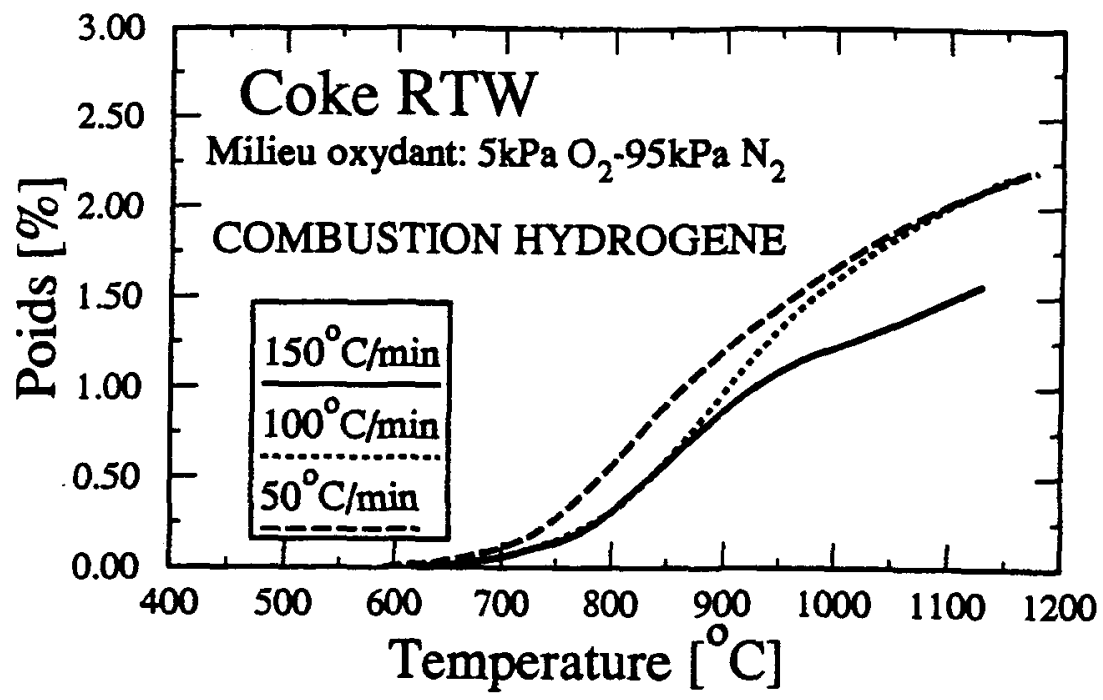

AnnexeVI.1 : Combustion de l'hydrogène du coke RTW.

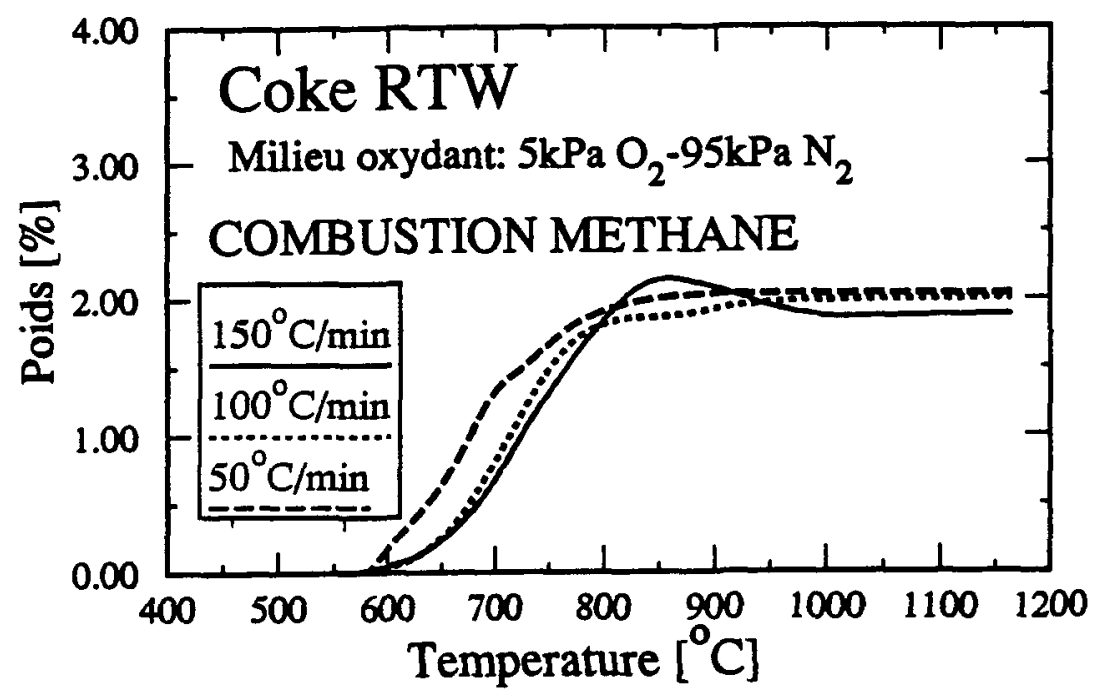

AnnexeVI.2 : Combustion du méthane du coke RTW. 


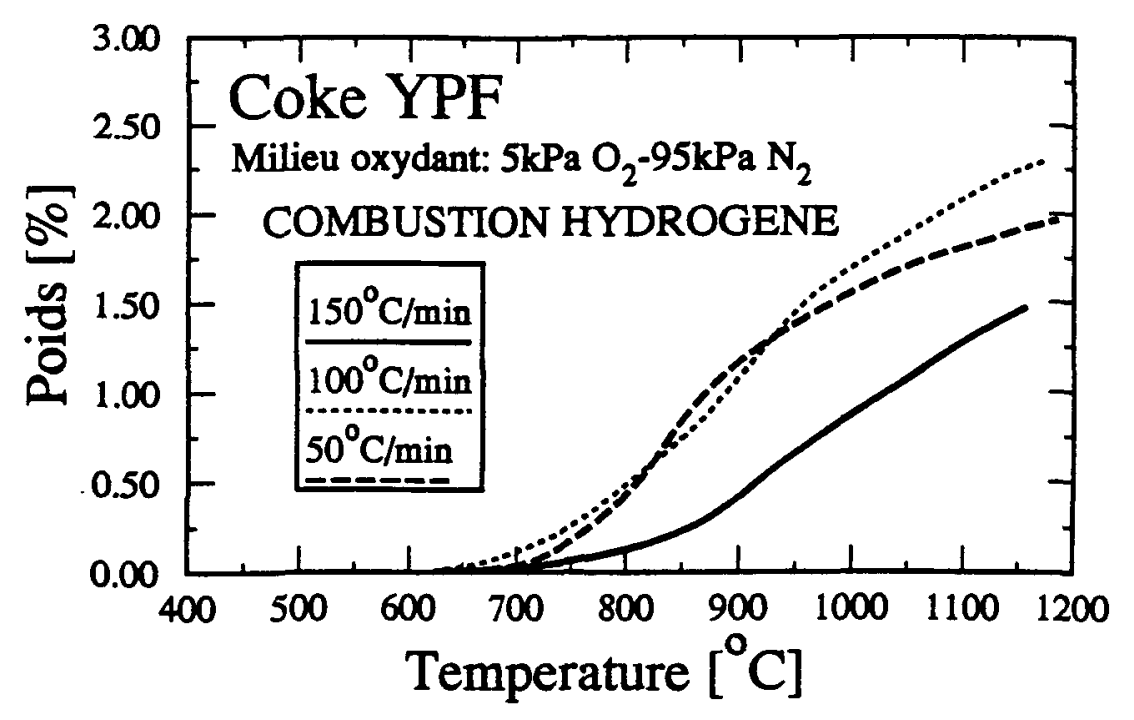

AnnexeVI.3 : Combustion de l'hydrogène du coke YPF.

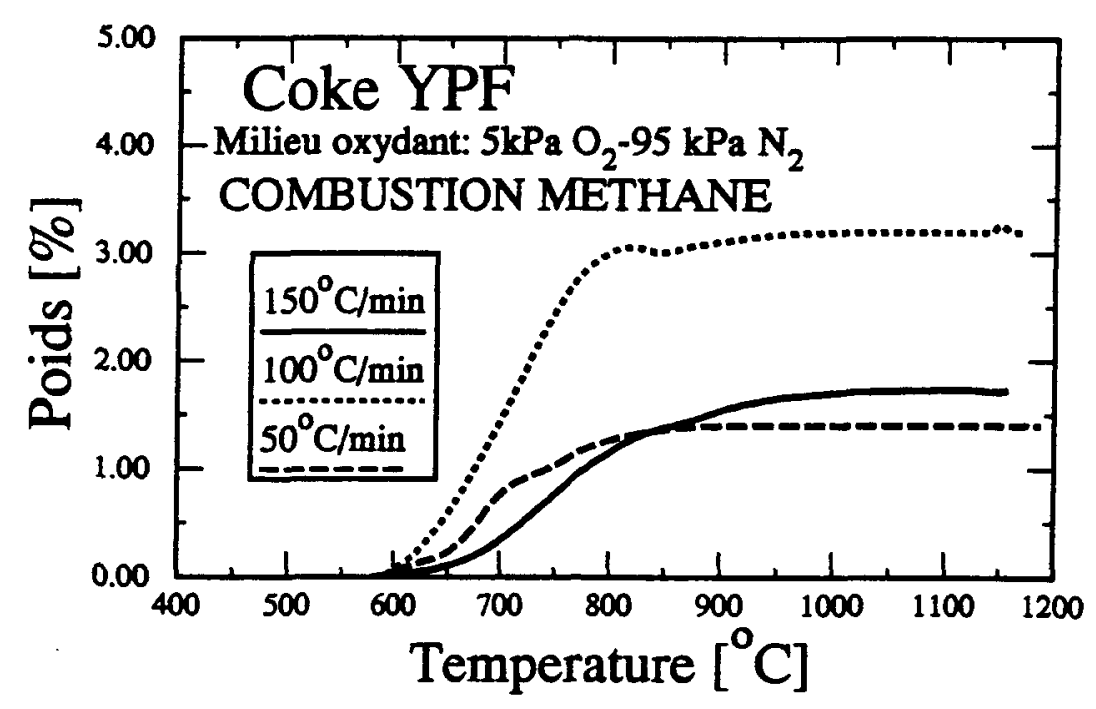

AnnexeVI.4 : Combustion du méthane du coke YPF. 


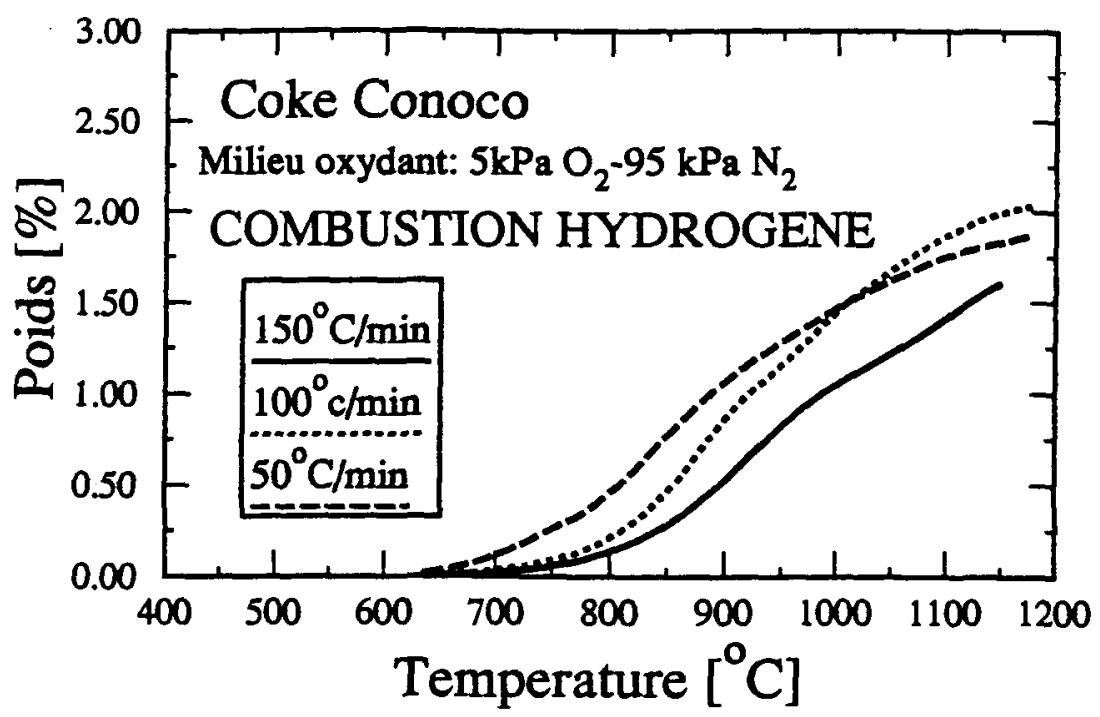

AnnexeVI.5 : Combustion de l'hydrogène du coke CONOCO.

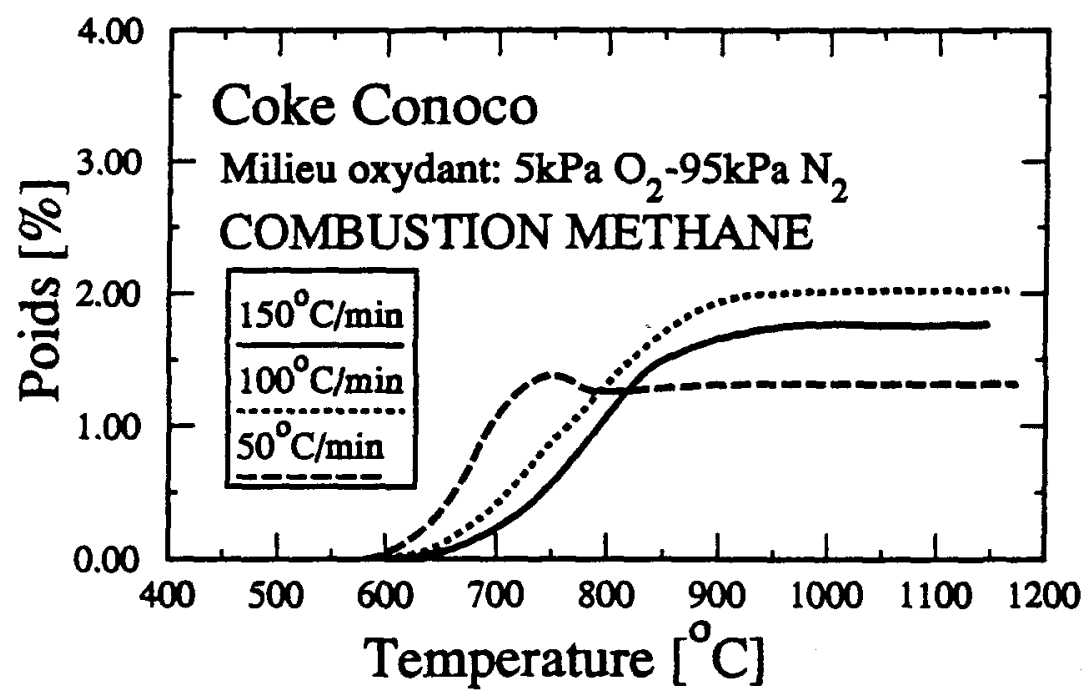

AnnexeVI.6 : Combustion du méthane du coke CONOCO. 
Annexe VII: Comparaison des résultats expérimentaux avec les rêsultats à l'équilibre, pour le coke TEXACO à $50^{\circ} \mathrm{C} / \mathrm{min}$. 


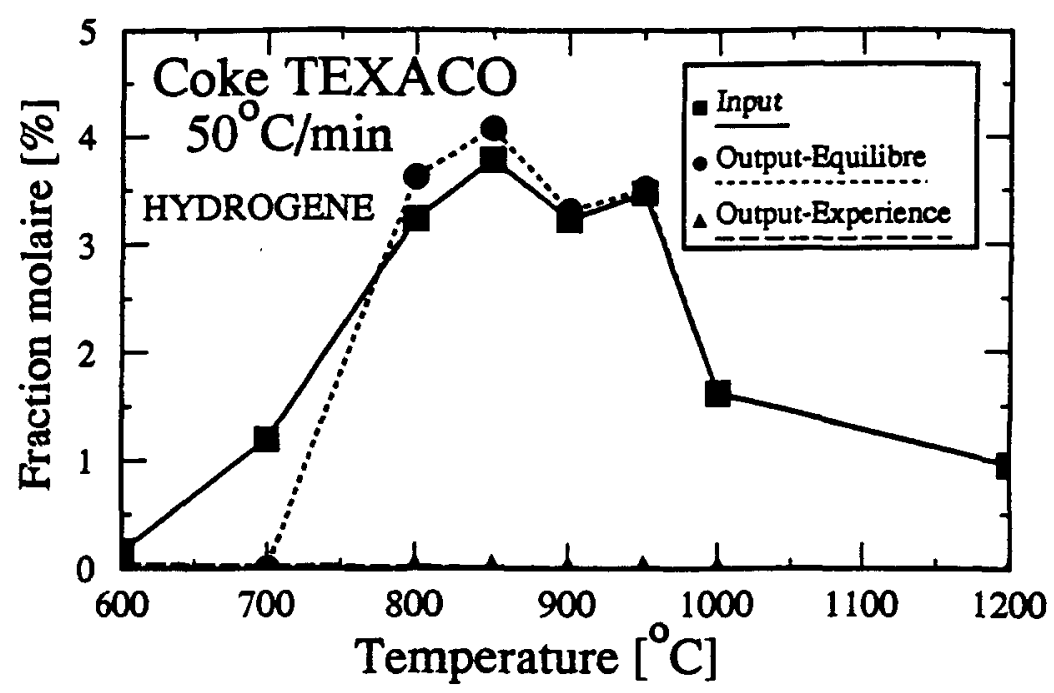

AnnexeVII.1 : Hydrogène du coke TEXACO à $50^{\circ} \mathrm{C} / \mathrm{min}$.

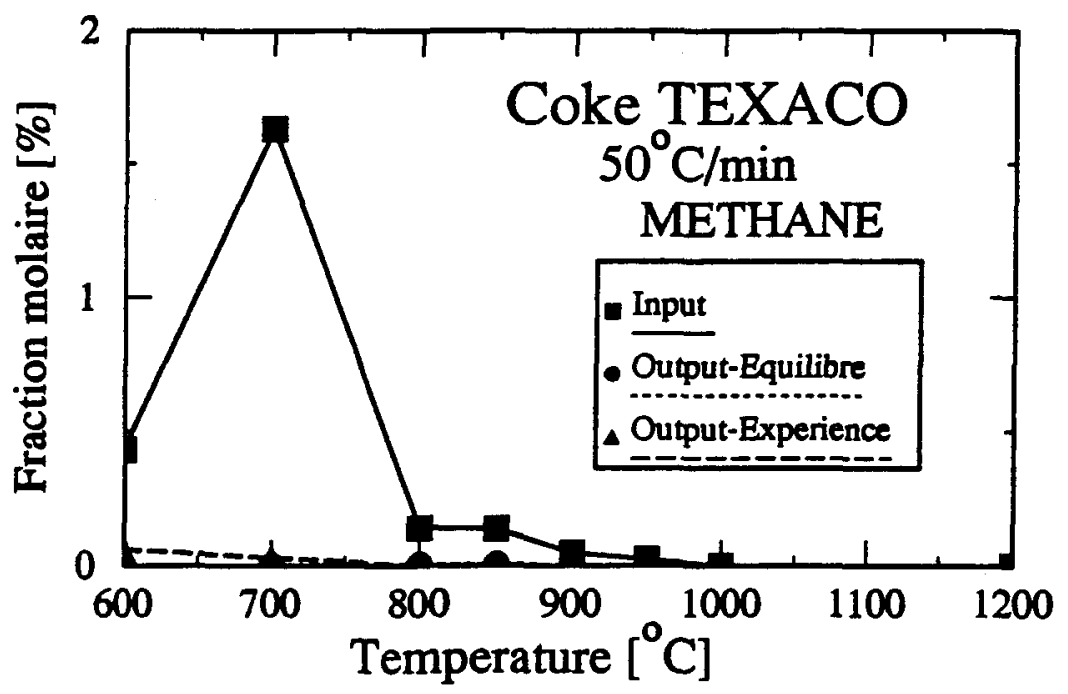

AnnexeVII.2 : Méthane du coke TEXACO à $50^{\circ} \mathrm{C} / \mathrm{min}$. 


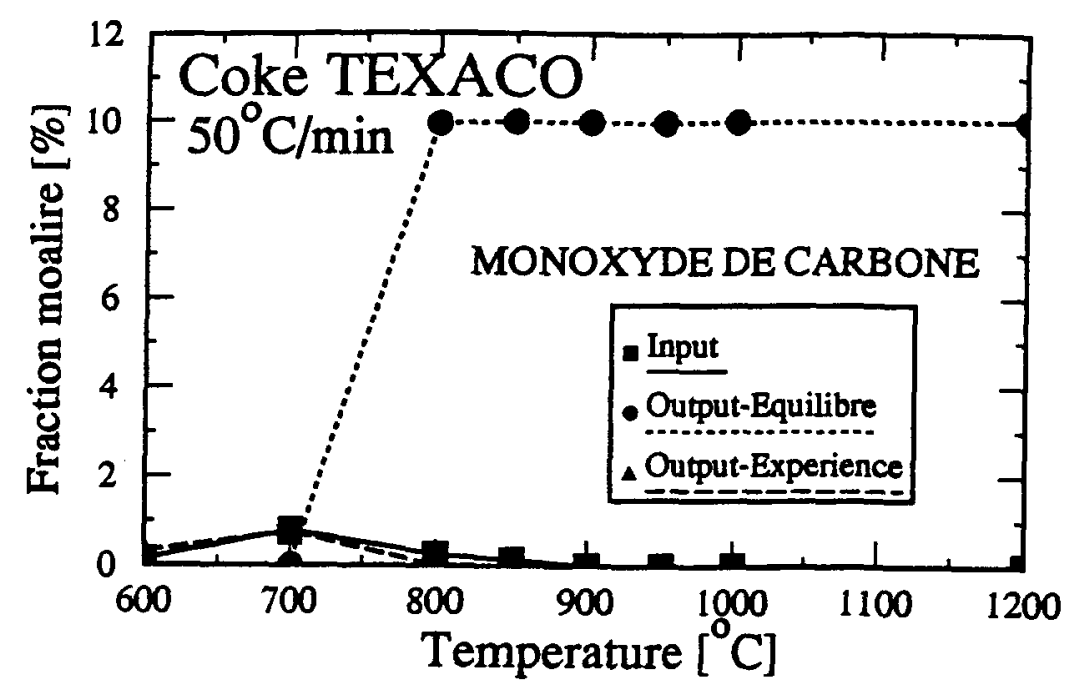

AnnexeVII.3 : Monoxyde de carbone du coke TEXACO à $50^{\circ} \mathrm{C} / \mathrm{min}$.

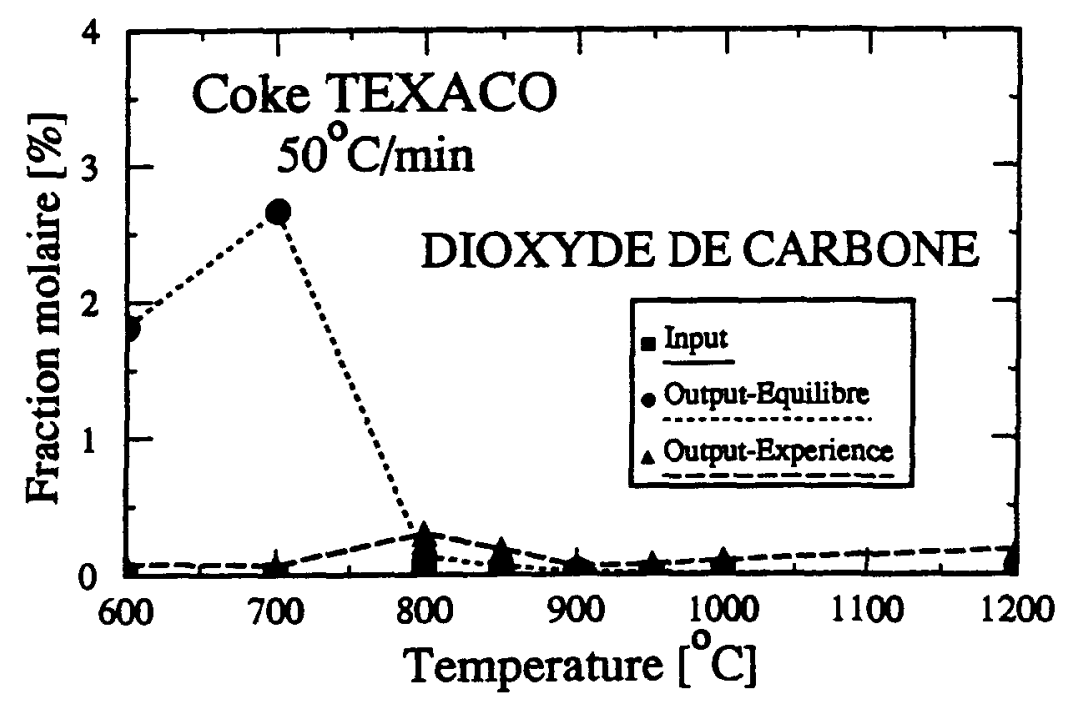

AnnexeVII.4 : Dioxyde de carbone du coke TEXACO à $50^{\circ} \mathrm{C} / \mathrm{min}$. 
Anneze VIII : Diagramme d'Arrhénius pour la dévolatilisation des trois autres cokes (RTW, YPF et CONOCO). 

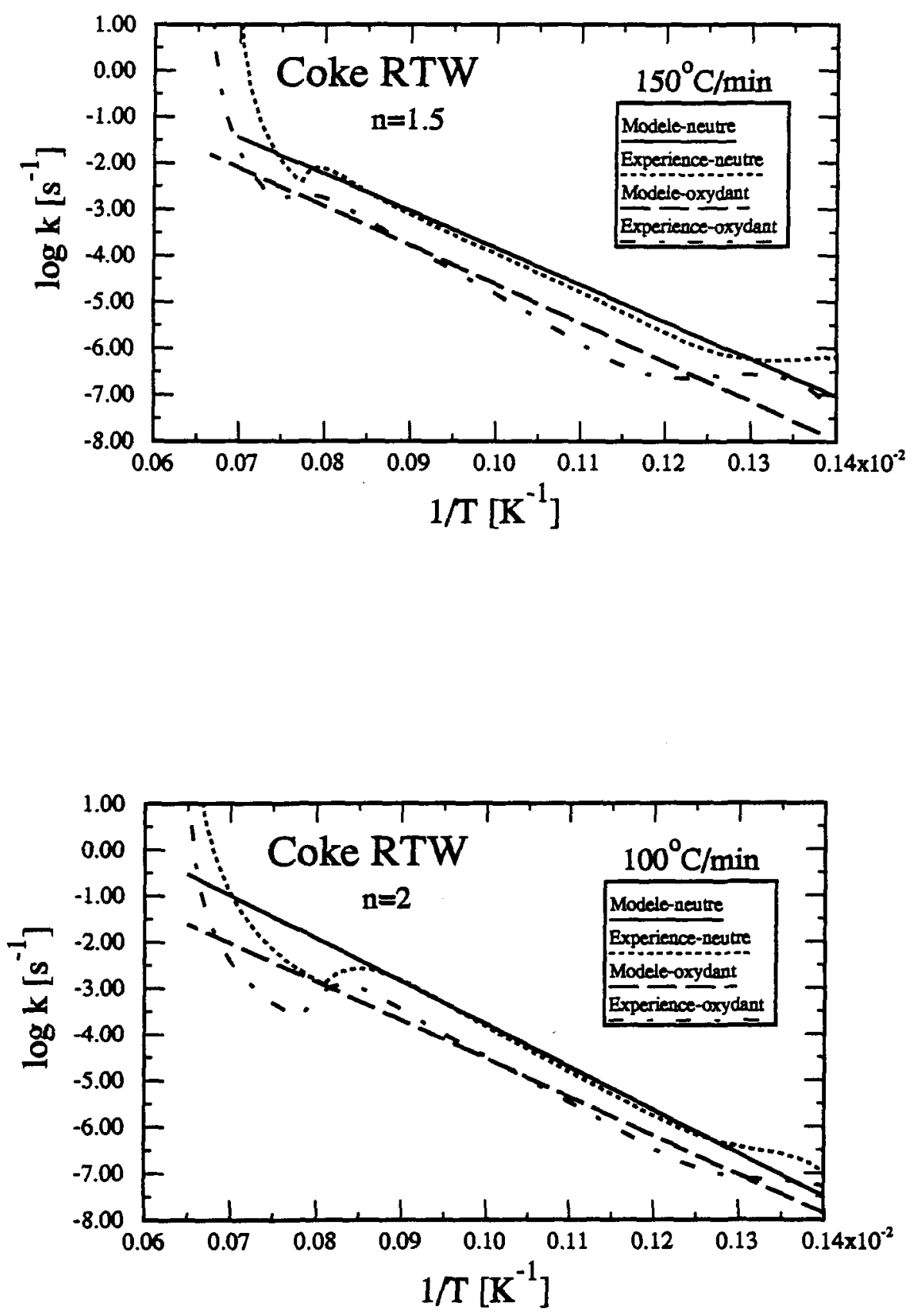

AnnexeVIII. 1 : Dévolatilisation du coke RTW en milieux oxydant ( $5 \%$ oxygène, $95 \%$ azote) et neutre $(100 \%$ azote). 

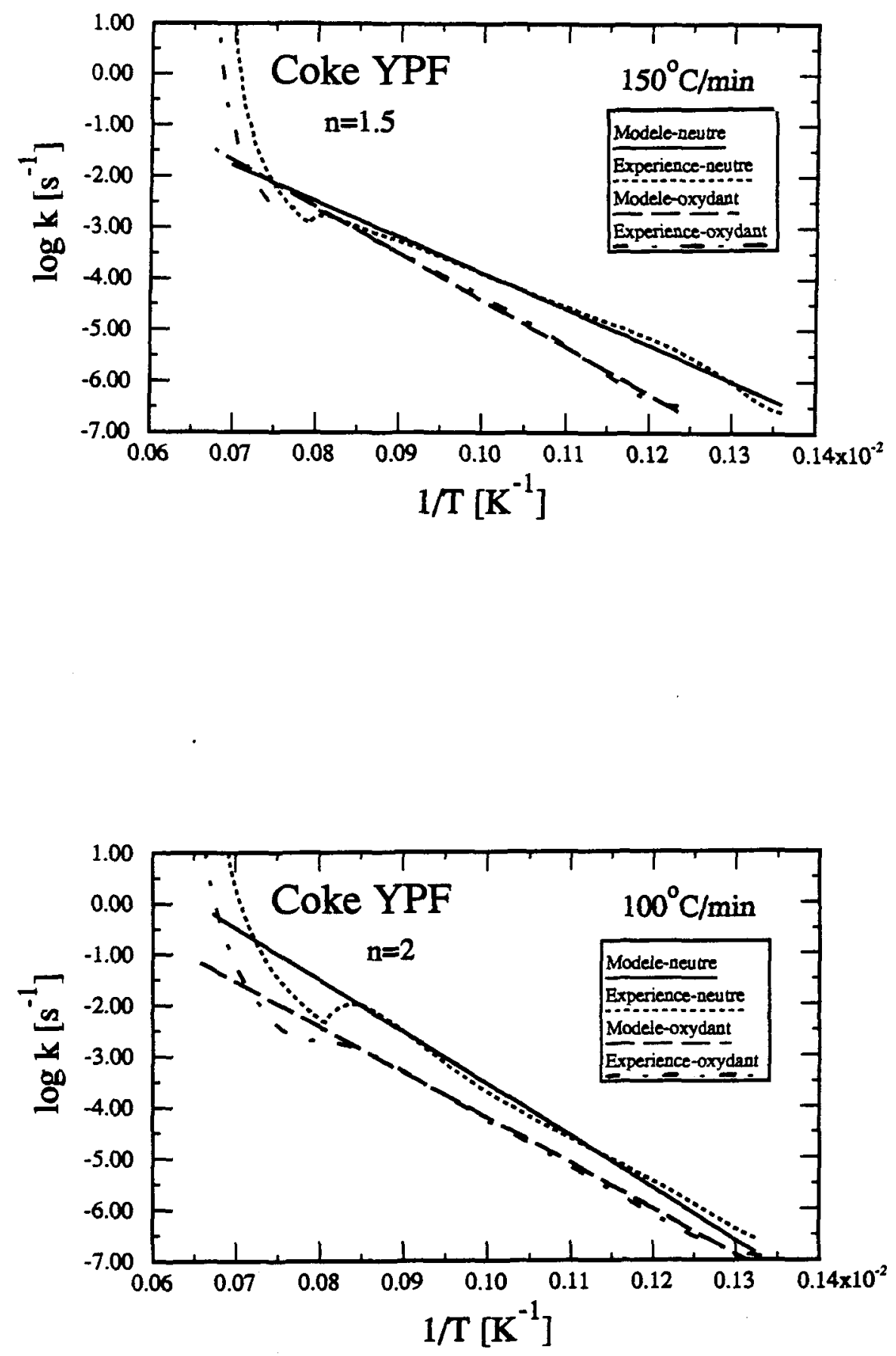

AnnexeVIII.2 : Dévolatilisation du coke YPF en milieux oxydant ( $5 \%$ oxygène, $95 \%$ azote) et neutre $(100 \%$ azote). 

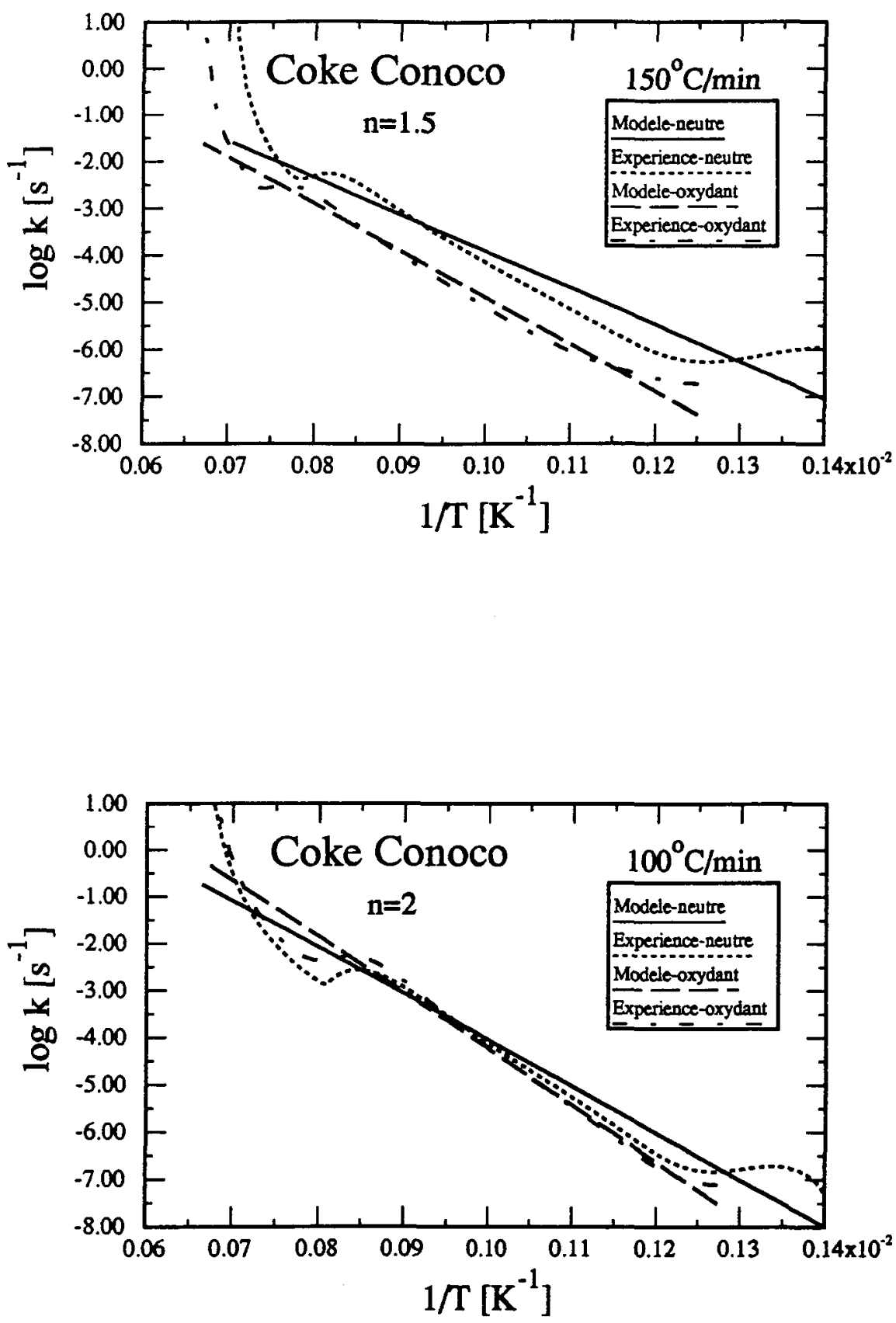

AnnexeVIII.3 : Dévolatilisation du coke CONOCO en milieux oxydant ( $5 \%$ oxygène, $95 \%$ azote) et neutre (100\% azote). 
Ce mémoire a été realisé à l'Université du Québec à Chicoutimi dans le cadre du Programme de Maitrise en Ingénierie 